\title{
Microscopic view of epitaxial metal growth: nucleation and aggregation
}

\author{
Harald Brune \\ Institut de Physique Expérimentale, Ecole Polytechnique Fédérale de Lausanne, \\ PHB-Ecublens, CH-1015 Lausanne, Switzerland
}

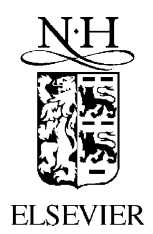

Amsterdam-Lausanne-New York-Oxford-Shannon-Tokyo 


\section{Contents}

1. Introduction 125

1.1. Growth as a non-equilibrium phenomenon $\quad 125$

1.2. Single atom displacements, nucleation, and mesoscopic morphologies 126

$\begin{array}{ll}\text { 1.3. Aggregation and pattern formation at surfaces } & 127\end{array}$

1.4. Kinetically controlled growth vs. sequential assembly of nanostructures 127

2. Surface diffusion and nucleation theory 129

2.1. Surface diffusion 130

2.2. Mean-field nucleation theory 132

2.3. Kinetic Monte-Carlo simulations 138

$\begin{array}{lr}\text { 2.3.1. Scaling of island size distributions } & 139\end{array}$

$\begin{array}{lr}\text { 2.3.2. Coalescence and island shape } & 140\end{array}$

$\begin{array}{ll}\text { 2.3.3. Scaling for coverages below and above saturation } & 141\end{array}$

3. Microscopic view of nucleation 143

$\begin{array}{ll}\text { 3.1. From nucleation to growth } & 144\end{array}$

3.1.1. Growth scenario for $i=1 \quad 144$

3.1.2. Analysis of island densities 145

3.2. Critical cluster size, dimer mobility, and Ostwald ripening $\quad 148$

$\begin{array}{ll}\text { 3.2.1. The critical cluster } & 148\end{array}$

$\begin{array}{ll}\text { 3.2.2. Dimer mobility } & 151\end{array}$

3.3. Consistency tests of nucleation and scaling theories 153

3.4. Growth at $D / F \ll 10^{5}$ - from post-deposition mobility to statistical growth 157

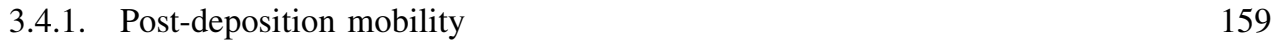

$\begin{array}{ll}\text { 3.4.2. Easy attachment } & 160\end{array}$

3.4.3. Statistical growth 161

3.5. Effect of isotropic strain on surface diffusion 163

3.6. Energy barriers and attempt frequencies from nucleation densities 167

$\begin{array}{ll}\text { 3.6.1. Surfaces with square symmetry } & 168\end{array}$

$\begin{array}{ll}\text { 3.6.2. Close-packed surfaces } & 170\end{array}$

3.6.3. Diffusion for systems with extremely small barriers 171

4. Nucleation on anisotropic and patterned substrates 173

$\begin{array}{ll}\text { 4.1. Nucleation on anisotropic substrates } & 173\end{array}$

4.1.1. Sticking anisotropy on fcc(1 10$) \quad 174$

4.1.2. Diffusion anisotropy on fcc(1 00$)$-hex 179

4.2. Nucleation and self-organization on patterned substrates 181

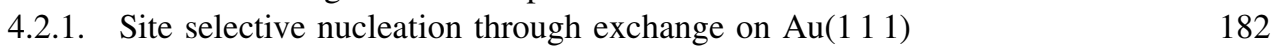

4.2.2. Adatom diffusion on $\mathrm{Au}(111)$ - the effect of dislocations 185

4.2.3. Nucleation on dislocation networks 186

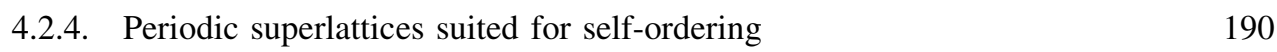

5. Aggregation and pattern formation at surfaces 194

5.1. Pattern formation in nature and modeling attempts 194

5.1.1. Non-equilibrium patterns and their common characteristics 194

$\begin{array}{ll}\text { 5.1.2. Shape transitions } & 197\end{array}$

$\begin{array}{ll}\text { 5.1.3. Modeling pattern formation } & 198\end{array}$

$\begin{array}{ll}\text { 5.2. Dendritic growth at surfaces } & 200\end{array}$

5.2.1. Crossover from random to dendritic growth 200 
5.2.2. Dendritic growth common to aggregation on hexagonal surfaces 202

5.2.3. Initial branching 204

5.2.4. The mechanism for dendritic growth 205

$\begin{array}{ll}\text { 5.2.5. General implications } & 208\end{array}$

5.2.6. No classical DLA clusters for metal aggregation 210

$\begin{array}{ll}\text { 5.3. Shape transitions at surfaces } & 211\end{array}$

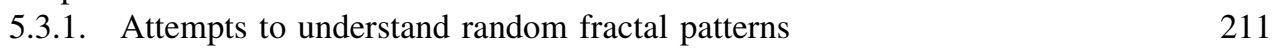

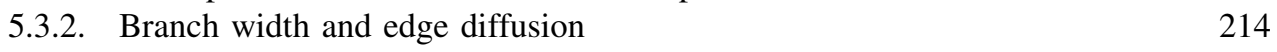

$\begin{array}{ll}\text { 5.3.3. Various polygonal island shapes } & 216\end{array}$

$\begin{array}{ll}\text { 6. Summary and outlook } & 220\end{array}$

$\begin{array}{ll}\text { Acronyms } & 221\end{array}$

Acknowledgements $\quad 222$

$\begin{array}{ll}\text { References } & 222\end{array}$ 



\title{
Microscopic view of epitaxial metal growth: nucleation and aggregation
}

\author{
Harald Brune ${ }^{1}$ \\ Institut de Physique Expérimentale, Ecole Polytechnique Fédérale de Lausanne, PHB-Ecublens, \\ CH-1015 Lausanne, Switzerland
}

\begin{abstract}
Thin films are often grown away from thermodynamic equilibrium and their morphology becomes determined by kinetics. The final structure of the epitaxial film is decided in the very early stage of submonolayer nucleation and island growth. Recent experiments with scanning tunneling microscopy opened up an unprecedented view of this early stage of epitaxial growth. Variable sample temperatures enabled quantification of the rates of the most important atomic diffusion events and tracing back their interplay yielding the final submonolayer morphology. The present understanding of nucleation and aggregation in light of these new experimental results is reviewed for the case of metals. Examples are given how the growth kinetics can be employed to create well-defined island morphologies and island arrays in a self-organization process. (C) 1998 Elsevier Science B.V.
\end{abstract}

Keywords: Epitaxy; Nucleation; Aggregation; Self-organization; Nanostructures; STM

\section{Introduction}

\subsection{Growth as a non-equilibrium phenomenon}

Epitaxial thin film growth is a non-equilibrium kinetic phenomena. In thermodynamic equilibrium all atomic processes proceed in opposite directions at equal rates, as implied by "detailed balance". This requires that adsorption from the gas phase must occur at the same rate as desorption, and clusters form at the same rate as they decay. At equilibrium there is hence no net growth and average macroscopic quantities such as surface coverage or roughness stay constant. The various microscopic surface processes then cause fluctuations around these equilibrium quantities which are usually well described by statistical mechanics. In order to obtain a net growth rate, one has to be away from equilibrium. The

\footnotetext{
${ }^{1}$ Tel.: +41 21 6933327; fax: +41 21 6933604; e-mail: harald.brune@ipe.dp.epfl.ch.
} 
degree to which growth proceeds away from equilibrium decides to which extent the morphology will be determined by thermodynamic quantities, such as surface and interface free energies, or by the growth kinetics.

Thin film growth is usually classified into three modes based on thermodynamic arguments [1]. The Frank-van der Merwe or two-dimensional (2D) growth is distinguished from the Volmer-Weber or three-dimensional (3D) growth. Two-dimensional growth is preferred if the sum of the surface free energy of the adsorbate and its interface free energy is larger than the substrate free energy. The Stranski-Krastanov mode is the intermediate case, where this energetic difference changes sign at a critical layer thickness provoking a transition from 2D to 3D growth. For heteroepitaxy this picture has to be refined by taking into account the strain energy arising from lattice mismatch [2].

Only in favorable cases can the desired growth morphology, for example layer-by-layer growth, be achieved within these thermodynamic limits. Very often, one is faced with the technical problem of growing flat layers, even though the film material does not wet the substrate. In addition, undesired effects that might set in at high temperatures, such as alloy formation, often necessitate to work far away from equilibrium. These conditions typically characterize molecular beam epitaxy (MBE), where a high supersaturation is applied in order to grow films rapidly from the vapor phase. Under such conditions growth is to a high degree determined by kinetics and the microscopic pathway taken by the system becomes decisive. Understanding and controlling growth morphologies in the kinetic regime therefore requires detailed knowledge of the microscopic processes involved. These processes are atomic diffusion events like terrace-, interlayer-, exchange- and edge-diffusion. These atomic displacements are thermally activated and their rates are generally well described by Boltzmann statistics. Measurements of the activation barriers and prefactors are required to determine the hierarchy of rates and thus to identify the rate limiting steps. Knowledge on these energetic barriers in turn allows one to predict and influence island and film morphologies.

\subsection{Single atom displacements, nucleation, and mesoscopic morphologies}

Over the recent past, considerable progress has been achieved in the understanding of how atomic processes contribute to thin film growth, as well as in quantifying their rates. On the experimental side, this is largely due to the extension of scanning tunneling microscopy (STM) [3,4] to variable substrate temperatures. This technique provides an unprecedented microscopic view of activated processes taking place on surfaces, such as chemical reactions or atomic diffusion. By tuning the temperature each process can be slowed down to the timescale which makes observation possible. From a study of the temperature dependence of the diffusivity the prefactor and activation energy can then be determined. A comparable view of atomic displacement processes was previously only obtained by field ion microscopy (FIM) [5-7] for a small number of elements.

Variable temperature STM was first applied to the kinetics of metal epitaxy, where it was demonstrated to close the gap between the observation of single adatom diffusion and the resulting film structure consisting of large islands. The evolution of a thin film from the formation of the first stable clusters, the initial increase in their density, their growth and finally coalescence could be followed for the first time in real space. Nucleation was explored under conditions where dimers are stable $(i=1)$ and immobile, allowing the first parameter free tests of nucleation theories [8-11] postulated long before MBE growth existed. This experimental development was accompanied by theoretical progress. In parallel with low temperature STM experiments, a self-consistent formulation of mean-field 
nucleation theory was published for the case of irreversible growth $(i=1)$ [12]. The results were compared to kinetic Monte-Carlo (KMC) simulations demonstrating that the rate equations from classical mean-field nucleation theory describe average quantities very well. Application of this theory to experiment provided a profound understanding of nucleation and growth. In addition, KMC simulations became a powerful tool to test ideas on how the interplay of the various microscopic processes determines the film and island morphology. Such simulations became widely accessible since the computing power required for producing sufficient statistics is available with today's desktop computers. As a result many aspects of thin film growth as island size distributions, kinetic roughening, and island morphologies have been addressed with technique.

\subsection{Aggregation and pattern formation at surfaces}

Access to atomic scale processes in real space and at low temperatures also opened up the possibility of examining pattern formation in two dimensions. There is considerable interest in the physics underlying fractal growth phenomena [13,14]. Several models exist aiming to explain these phenomena since quite some time. The classical model of fractal growth is the diffusion limited aggregation (DLA) scenario implying irreversible sticking to the site of first attachment $[15,16]$. Low temperature metal aggregation on a close-packed surface was for a long time assumed to be the experimental realization of DLA. Recent experiments, however, show that fractal objects with well-defined symmetry form instead [17], and the mechanisms underlying their formation were discovered only recently. These results can contribute to a general understanding of the transition from random to ordered (dendritic) interfacial patterns which characterizes a variety of non-equilibrium growth processes in nature, such as electrochemical depositon, crystal growth, and the growth of bacterial colonies.

\subsection{Kinetically controlled growth vs. sequential assembly of nanostructures}

The increasing understanding of nucleation and pattern formation enables the laws of nature to be exploited with the goal being the design of well-defined, small-scale structures on surfaces. Besides self-organized growth in the kinetic regime, there are different approaches for the nanostructuring of surfaces, each having specific advantages and limitations. First of all, clusters with well-defined sizes can be created in the gas phase and subsequently landed onto a substrate in a controlled non-destructive manner [18]. Arbitrary atomic scale structures could be assembled with the tip of an STM, either through direct displacement of atoms [19,20], or through tip-assisted decomposition of chemical species [21]. The principal drawback of scanning probe based methods is their serial character meaning that only one such structure can be built at a time. As long as the interesting physical properties of such nanostructures, e.g. magnetism, cannot be accessed on an atomic scale, a large density and probably also high uniformity of such structures is required to gain access with integrating techniques. A high abundance of such structures, ranging from mono-atomic chains to fractal or compact islands of any size, can be created by self-assembling techniques in parallel processing [22]. ${ }^{2}$ The drawback here is the limited degree of control one has over the atomic displacements participating in nucleation and

\footnotetext{
${ }^{2}$ Self-organization is applied in semiconductor growth in the thermodynamic regime to grow quantum dots through StranskiKrastanov islanding [23,24].
} 
aggregation at surfaces. These processes are governed by statistics which reflect themselves in the variance of island size and distance distributions. Having said this, however, we will see below how a detailed knowledge of the processes involved, together with the proper choice of the substrate, can help to considerably reduce statistical broadening, presently rendering self-organized growth quite attractive for the creation of nanostructures.

The scope of the present article is to review recent experiments which lead to an improved understanding of the kinetics of metal epitaxy and pattern formation on surfaces. We will also show directions in which this knowledge can be applied, with the goal of creating nanostructures of controlled size, dimension, and uniformity. We focus on work performed on metallic systems. For recent advances in the kinetics of semiconductor MBE the reader is referred to Refs. [25-27]. For simplicity, we do not include systems where adsorption leads to intermixing and surface alloy formation [28]. For the sake of comparison with theory and for quantitative understanding we focus on the simplest cases, i.e. nucleation and aggregation on isotropic substrates, at temperatures where dimers are stable. However, we also address more complicated cases, like nucleation on anisotropic and inhomogeneous substrates. The latter have their own charm. These substrates reveal highly symmetric periodic patterns, resulting from long range interactions, either between dislocations or between atoms in moiré patterns. These patterns can be transferred via kinetics to an island population yielding superlattices of almost mono-dispersed islands. Throughout the work presented in this review the role of strain in surface diffusion will also be addressed, however, only briefly, since a full account was given in a recent book chapter [29].

The organization of the paper is as follows. In Section 2 we give a brief introduction into mean-field nucleation theory and compare self-consistent solutions to approximations formerly proposed in the literature. We then introduce KMC simulations and show the strengths and limitations of mean-field theory with respect to such simulations. This section intends to provide the reader who is not familiar with nucleation theory with the necessary background to quantitatively analyze experimental data such as nucleation island densities in terms of surface diffusion barriers. Section 3 is a survey of nucleation on isotropic metal surfaces studied experimentally by variable temperature STM and analyzed with mean-field theory and KMC simulations. The system $\mathrm{Ag} / \mathrm{Pt}\left(\begin{array}{lll}1 & 1 & 1\end{array}\right)$ will be used as model to illustrate the various phases of nucleation and growth in the first two sections. We then present several experimental tests of nucleation and scaling theories. For some experimental examples we compare the analysis by means of nucleation theory with that by means of $\mathrm{KMC}$ in order to demonstrate the consistency of both methods. We will show that our present understanding of nucleation on isotropic substrates allows us to precisely measure diffusion parameters from island densities obtained under conditions where dimers are stable. We give an example where this method was applied to study the effect of strain on diffusion in Section 3.4. This section on isotropic substrates is summarized by comparing migration barriers obtained from nucleation studies with results from FIM and theory. We then turn in Section 4 to nucleation on anisotropic and inhomogeneous substrates. Examples for the first will be fcc(1 10$)$ and hex-reconstructed fcc(100) surfaces. Inhomogeneous substrates represent surfaces where diffusion barriers and binding energies vary upon translation. Typical candidates are substrates with dislocations. Quantitative studies on the effect of dislocations are presented, the most spectacular examples being dislocation networks, as their periodicity can be transferred to grow regular island arrays. Section 5 is devoted to aggregation and the resulting pattern formation and shape transitions at surfaces. We first give an introduction to fractal growth phenomena appearing in nature and introduce fractal measures as well as describe shortly modeling of fractal growth. We then turn to 
pattern formation observed for metal aggregation on fcc(111) surfaces. We first focus on the mechanism leading to the formation of symmetric patterns, which is now well understood. The formation of random patterns is far from being understood. We discuss present ideas that attempt to explain these structures. Finally, we discuss shape transitions observed for compact islands on fcc(llll) surfaces. The present review is closed by a brief summary and an outlook in Section 6. The intention of this final section is to show up future directions of research in the field of physics and chemistry of small-scale metal particles adsorbed on metal substrates.

\section{Surface diffusion and nucleation theory}

In the growth of thin films from the vapor phase surface migration of single adatoms is the most fundamental of all the diffusion processes. It gives rise to nucleation of islands on substrate terraces or to step flow growth at elevated temperatures. One distinguishes the collective diffusion coefficient (also known as chemical or Fickian diffusion coefficient) of an ensemble of mutually interacting particles from the tracer (or intrinsic) diffusion coefficient describing the mean square displacement of one isolated random walker per unit time. The chemical diffusion is commonly measured by the decay of concentration profiles or by looking at density fluctuations of a lattice gas induced by the mobility of the (often repulsively) interacting particles (field emission fluctuation method [30], see also detection of momentum transfer due to particle motion in inelastic He scattering [31]). Recently STM was applied to measure flicker noise in the tunneling current due to diffusing adatoms passing the tunneling gap $[32,33]$.

The second type of diffusion, trace diffusion, has tradionally been studied by means of field ion microscopy (FIM) where the migration path of single atoms (or clusters), adsorbed on single crystal facets of the FIM tip, can be traced at low temperatures [5-7,34]. Very recently, tracer diffusion has also been followed through direct inspection of the adatoms' mean square displacement by means of STM [35-37]. However, when quantifying diffusion rates obtained from in situ STM observations, one generally has to worry about possible tip-sample interactions. ${ }^{3}$ A theoretical study showed that diffusion barries are reduced in the region under the STM tip [39]. This is consistent with experimental observations made for a number of systems [37,40]. In general, the tip influence depends on the tip-tosample distance which is controlled by the tunneling resistance. A recent study for Pt diffusion on $\operatorname{Pt}(110)-(1 \times 2)$ showed that the tip influence becomes negligible for large tunnel resistances $\left(R_{\text {gap }}>5 \times 10^{7} \Omega\right)$ [41]. The tip influence has to be compared with the magnitude of the diffusion barrier. Generally, small effects are expected for systems with large barriers, e.g. dimer diffusion on $\operatorname{Si}\left(\begin{array}{lll}1 & 0 & 0\end{array}\right)$ [35], whereas in situ observations of diffusion on close-packed metal surfaces might get strongly perturbed by tip effects [37].

An alternative way to quantify tracer diffusion is to measure island densities that form upon deposition onto a single crystal surface as a function of temperature. Strictly speaking, vapor phase epitaxy is characterized by chemical diffusion, as there are many monomers present at a time, which might be subject to mutual interactions. For typical growth rates $\left(D / F>10^{5}\right.$, where $D$ is the diffusion rate and $F$ stands for the deposition flux) the density of diffusing particles is however rather small

\footnotetext{
${ }^{3}$ A dramatic example was reported by Li et al. [38] for $\operatorname{Ag}(110)$, where tip-sample interactions can lead to displacements of atomic steps, even at tunneling currents in the sub-nA range.
} 
$\left(n_{1}<10^{-3}\right.$ islands per adsorption site). The mean inter particle distance is rather large compared to typical interaction ranges for metal adatoms [42,43]. Therefore nucleation takes place in the low coverage limit where mutual interactions are negligible and hence the chemical diffusion coefficient equals the tracer diffusion coefficient [30].

The activation energy, $E_{\mathrm{m}}$, and attempt frequency, $\nu_{0}$, for surface diffusion can be extracted from the temperature dependence of island densities, for example by application of mean-field nucleation theory. Before we proceed to a comparison of nucleation theory with STM results for island densities in Section 3, we first define in this section the tracer diffusion coefficient, $D$, and give an introduction to mean-field nucleation theory. We restrict ourselves to the simplest case where dimers are stable and the substrate is isotropic. This case, the so-called irreversible growth, formerly was a mere theoretical example and has now become experimentally accessible. It has served as a model case to test nucleation theory and probably will take on a central role in the determination of surface diffusion parameters. For determination of diffusion parameters from measured island densities one either applies a scaling law from nucleation theory which is valid at or close to saturation coverage. Below this coverage, one has to integrate rate equations describing nucleation and growth. In the latter case one needs to put in expressions for capture rates. These capture rates have been discussed in detail in the literature (see for instance Ref. [10]). Here we give the expression for the most relevant approximations and address their strengths and limitations. We also include recent results that were obtained in solving the uniform depletion approximation self-consistently [12]. The calculations present the most precise approach available from mean-field theory.

Due to the rapid increase in computational resources during the last decade, kinetic Monte-Carlo (KMC) simulations have become accessible to many groups. These simulations have served as a useful tool to test ideas on nucleation and aggregation, as will be demonstrated for several examples within this review. We therefore give a brief introduction to this technique and show that average quantities obtained from mean-field theory are fully consistent with results from KMC. Other than mean-field theory, these simulations correctly describe correlations between islands and thus their coalescence. Island correlation effects become particularly important at larger coverage. We will investigate in which coverage range with which precision the scaling laws of nucleation theory are valid. We also discuss how these laws become modified by the effect of island shape and coalescence.

\subsection{Surface diffusion}

For thermally activated diffusion the hopping rate of an adatom on a periodic substrate may be expressed according to transition state theory (TST) [44] as

$$
\nu=\frac{k T}{h} \exp \left(\frac{-\Delta F}{k T}\right)
$$

where $\Delta F$ is the difference in the Helmholtz free energy between the saddle point (transition state) and the minimum (binding site) of the potential energy surface $(k, h$, and $T$ are the Boltzmann constant, Planck's constant, and the substrate temperature). It is convenient to separate the entropy and the energy terms, yielding

$$
\nu=\nu_{0} \exp \left(\frac{-E_{\mathrm{m}}}{k T}\right) \quad \text { with } \nu_{0}=\frac{k T}{h} \exp \left(\frac{\Delta S_{\mathrm{vib}}}{k}\right),
$$


where $\nu_{0}$ is commonly referred to as the attempt frequency, and $E_{\mathrm{m}}$ as the migration barrier. In order to interpret experiments in terms of $E_{\mathrm{m}}$ and $\nu_{0}$ we need these two quantities to be temperature independent. Let us first address this point for $E_{\mathrm{m}}$. In calculations, one distinguishes static barriers from dynamic ones. The former contain only the difference in potential energy $\Delta V$ between transition state and adsorption minimum. Dynamic barriers take account of the vibrational internal energy difference

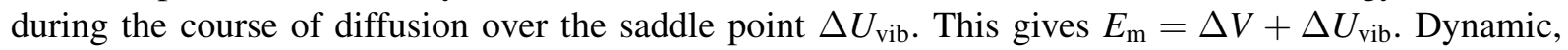
temperature dependent values of the barrier are typically inferred from molecular dynamics simulations [45,46], or within harmonic approximations for the lattice dynamics [47]. At low temperatures, $\Delta U_{\text {vib }}$ is small compared to $\Delta V$, so for many purposes it suffices to assume $E_{\mathrm{m}}$ to be temperature independent, and to assign it with the static barrier. Such barriers are for instance reported from ab initio calculations performed at $0 \mathrm{~K}$.

It is important to note that the attempt frequency is also temperature independent, to a very good approximation. The decrease in $\Delta S_{\text {vib }}\left(\Delta S_{\text {vib }}<0\right)$ in the exponential of Eq. (2a) compensates the linear increase of its prefactor with increasing temperature. For the $1 \mathrm{D}$ case, this follows when considering the partition functions at the binding and transition site [48], $Q_{\mathrm{b}}$ and $Q_{\mathrm{tr}}$, respectively:

$$
\exp \left(\frac{\Delta S_{\mathrm{vib}}}{k}\right)=\frac{Q_{\mathrm{tr}}}{Q_{\mathrm{b}}}, \quad \frac{Q_{\mathrm{tr}}}{Q_{\mathrm{b}}}=1-\mathrm{e}^{-h \nu_{\mathrm{vib}} / k T} \approx \nu_{\mathrm{vib}} h / k T, \quad \text { therefore } \nu_{0} \approx \nu_{\mathrm{vib}} .
$$

For the 2D case one might refer to MD simulations which show that $\nu_{0}$ is constant over a large range of temperatures [47]. Eq. (2b) relates the attempt frequency for surface diffusion with the vibrational frequency of the adatom in its binding site, being of the order of $10^{12} \mathrm{~s}^{-1}$. Experimental observations of tracer diffusion confirm that the prefactor and energy barrier are constant as they yield straight lines in Arrhenius representations of the mean square displacements; the example with best statistics is $\operatorname{Ir} / \operatorname{Ir}\left(\begin{array}{lll}1 & 1 & 1\end{array}\right)$ [34].

The mean square displacement of the random walker per unit time, or the tracer diffusion coefficient $D$ is inferred from the hopping rate $\nu$ by the Einstein relation

$$
D=\frac{1}{2 d} \nu
$$

where $d$ is the dimension of the motion. (Note that tracer diffusion is normally labeled $D^{*}$ whereas $D$ stands for chemical diffusion coefficients; since we only consider tracer diffusion, respectively, chemical diffusion in the limit of extremely low coverages, we have a single notation $D$.) In the present paper we consider motion on the atomic scale. It is therefore convenient to express surface areas in terms of substrate unit cells. Then $D$ becomes the number of unit cells visited by the adatom per unit time. $D$ is often divided into a prefactor, $D_{0}$, and a Boltzmann factor, giving, from Eq. (2a)

$$
D_{0}=\frac{1}{2 d} \nu_{0} \quad \text { and } \quad D=D_{0} \exp \left(\frac{-E_{\mathrm{m}}}{k T}\right)
$$

It is the tracer diffusion coefficient defined by Eq. (3) that enters into nucleation theory. The way in which the monomer diffusion rate, $D$, is linked to nucleation island densities is the subject of the next section. The detailed knowledge of this relation enables the determination of precise values for $E_{\mathrm{m}}$ and $\nu_{0}$ from experiments monitoring the island density as it varies with temperature. 


\subsection{Mean-field nucleation theory}

In the course of deposition, the atoms arriving from the gas phase with a rate $F$ (units are atoms per surface unit cell and second, i.e. $\mathrm{ML} \mathrm{s}^{-1}$ ) diffuse with rate $D$ until they meet a second (diffusing) adatom and create a dimer. Let us assume for the present purpose, we perform our experiment at sufficiently low temperature that this dimer rests stable and immobile. As deposition proceeds, the number of dimers will increase roughly linearly until their concentration $n_{2}$ becomes comparable to the density of monomers $n_{1}$ (also densities are expressed in atoms per unit cell, i.e. in ML). From there on, the probabilities of a diffusing monomer to encounter one of its own or a dimer become comparable and cluster growth competes with the creation of new stable nuclei (dimers in our example). After the density of stable nuclei $n_{x}$ ( $x$ standing for any size that is stable) has increased sufficiently, any further deposition will exclusively lead to island growth. At this saturation island density, the mean free path of diffusing adatoms is equal to the mean island separation and adatoms will attach themselves with much higher probability to existing islands then to create new ones. Approaching coverages of about half a monolayer (ML), islands eventually coalesce which decreases their density.

This simple scenario proves to be correct, as demonstrated in recent experiments to be discussed in Section 3. Nucleation theories relying on this scenario had been proposed long before this experimental information became available, even before MBE existed. Theoretical work on the mechanism of crystal growth dates back to the work by Frenkel in 1924 [49]. Other early contributions are those of Volmer [50], Kossel [51] and Stranski [52]. Forty years later, Zinsmeister [8,9] put down rate equations quantitatively describing this scenario. Much of the work that followed focused on the solution of these rate equations for the various cases of supersaturation and island dimensionality. It is out of the scope of the present work to provide a general survey on mean-field nucleation theory, excellent reviews of which exist by Venables et al. [11], Lewis and Anderson [53], and Stoyanov and Kashchiev [54]. For the experimental work to be discussed below, we are interested in the very simple case of nucleation and growth of 2D islands with dimers being stable and immobile and no re-evaporation occurring (for the more general case the reader is referred to Ref. [10]). The rate equations for the density of monomers and stable islands for that case read

$$
\begin{aligned}
\frac{\mathrm{d} n_{1}}{\mathrm{~d} t} & =F-2 \sigma_{1} D n_{1}^{2}-\sigma_{x} D n_{1} n_{x}-\kappa_{x} F\left(F t-n_{1}\right)-2 \kappa_{1} F n_{1}, \\
\frac{\mathrm{d} n_{x}}{\mathrm{~d} t} & =\sigma_{1} D n_{1}^{2}+\kappa_{1} F n_{1} .
\end{aligned}
$$

The terms on the right-hand side of Eq. (5) denote, respectively, the increase of monomer density due to deposition with flux $F$, its decrease due to the encounter of two diffusing adatoms resulting in the creation of a dimer (associated with the disappearance of two atoms), the decrease occurring when a monomer is captured by a stable island, and finally two terms which denote the decrease caused by direct impingement onto stable islands and monomers. In Eq. (6) the terms on the right-hand side account for the increase of stable island density, $n_{x}$, due to the creation of dimers, first when two monomers meet by diffusion, and second upon direct deposition onto an adatom. In these equations coalescence is neglected; incorporation would add a further term $-2 n_{x}\left(F-\mathrm{d} n_{1} / \mathrm{d} t\right)$ to Eq. (6) [11].

A central point to note with these rate equations is that they express the time evolution of the average values of $n_{1}$ and $n_{x}$. All information on the local distribution of monomers $n_{1}(\mathbf{r}, t)$ is contained in the capture rates $\sigma_{1}$ and $\sigma_{x}$, which involve solving a Helmholtz type diffusion equation for monomers in 
two dimensions in the presence of a certain density of stable islands. The monomer concentration decreases in the vicinity of islands due to capture. The capture rates are proportional to the gradient of the monomer concentration at the island edges. The theoretical work focuses on the solution of this diffusion problem and the coupled differential equations, of which Eqs. (5) and (6) represent simple examples. In general this problem is solved in the mean-field assumption, that is, outside the islands the monomer density immediately takes on its average value. The time evolution of island and monomer densities can be obtained from integration of these equations, and this we will do below.

Very often, one is interested in the saturation island density, as this reflects the mean free path for monomer diffusion. The temperature dependence of this quantity thus allows one to extract information on surface diffusion. In principle, one can integrate Eqs. (5) and (6) until saturation of $n_{x}$, which requires detailed knowledge on the capture rates. However, there are also equations that directly relate $n_{x}$ at saturation and $D[10,55]$. A good understanding for the power law expressed by these equations can be derived by simple arguments given for example by Villain et al. [56,57] in their "minimal model". They obtain the following result for the surface diffusivity:

$$
\frac{D}{F} \approx \frac{l^{6}}{\ln \left(l^{2}\right)} \text {. }
$$

The characteristic length, $l$, can either be identified with the mean island distance, or with the mean free path of diffusing adatoms before they create a new nucleus or are captured by existing islands. The logarithmic correction term appearing in the denominator of Eq. (7) is small [10]. Omitting this term yields $l \approx(D / F)^{1 / 6}$. The central result of Eq. (7) is that $l$ depends only on the ratio $D / F$. This is due to the fact that the flux is the only quantity introducing time, if there is no re-evaporation and dimers are stable. Thus the ratio of deposition to diffusion rate determines the mean free diffusion path and the mean island distance attained at saturation.

The minimal model omits pre-exponential numerical factors, which can vary between $10^{-2}$ and 10 , however (depending on the regime of condensation, the coverage, and the critical nucleus size; see Fig. 6 in Ref. [11]). The result containing these quantities represents the central prediction of nucleation theory $[10,55]$. Here we give the expression for the island density and for the more general case of larger stable clusters, still for $2 \mathrm{D}$ islands and complete condensation. ${ }^{4}$ It reads

$$
n_{x}=\eta(\Theta, i)\left(\frac{D}{F}\right)^{-\chi} \exp \left(\frac{E_{i}}{(i+2) k T}\right), \quad \text { with the scaling exponent } \chi=\frac{i}{i+2} .
$$

In Eq. (8), $i$ denotes the critical cluster size, and $E_{i}$ its binding energy $\left(E_{1}=0\right)$. In a microscopic picture, which is most appropriate for the results to be discussed, a critical cluster turns into a stable one upon incorporation of one extra atom. Stable and critical are dynamically defined, i.e. stable refers to a cluster that grows more rapidly than it decays during deposition. ${ }^{5}$ From Eq. (8) it is clear that measuring $D$ is most conveniently done at temperatures and deposition fluxes where dimers are stable, since then $n_{x} \propto(D / F)^{-1 / 3}$. In principle, one can then extract $E_{\mathrm{m}}$ and $\nu_{0}$ for surface migration of single adatoms in a straightforward way from the slope and intercept of a line fitted to the experimental island

\footnotetext{
${ }^{4}$ The general form of Eq. (8) describing the three regimes of nucleation can be found as Eq. (29) in Ref. [55].

${ }^{5}$ In that sense the critical nucleus size is that value which produces the lowest nucleation density for a given $F$ and $D, i$ is thus determined as an output, and not an input, of the calculation [55,58]. In the thermodynamic picture, the critical cluster size is given by the maximum of the Gibbs free energy [11].
} 
densities in an Arrhenius plot. We will see below to what extent this approach is applicable, and in which range of $D / F$ and with what precision the power law expressed in Eq. (8) holds.

Self-consistency requires that integration of the rate equations (5) and (6) up to saturation yields island densities obeying Eq. (8). The capture numbers $\sigma$ appearing in the equations require the solution of a 2D diffusion problem for the monomer density in the presence of the various islands. This problem has an exact solution only for the high temperature case of incomplete condensation where evaporation is dominant. For the low temperature case of complete condensation various approximations for $\sigma$ have been proposed [10]. One of which is the uniform depletion approximation [10]. Recently, this approximation was solved self-consistently and shown to yield exact coincidence with KMC simulations for average quantities such as island densities [12]. The self-consistent solutions of [12] certainly represent one of the best approximations to the capture rates, however, they require a considerable calculational effort. For simplicity, one might therefore want to use the uniform depletion approximation in its simplest form or one of the other approximations given in the original literature. Fig. 1(a) shows a comparison of the approximations to the scaling law expressed in Eq. (8). We have selected a coverage of $0.12 \mathrm{ML}$, which is close to saturation, and assumed $\eta=0.25$ in Eq. (8) (see curve for $i=1$ in Fig. 6(c) of Ref. [11]).

The first assumption for the capture rates that comes into mind is the geometric concept [9], where capture rates are proportional to the island perimeter seen by the approaching monomers. This concept has been applied to fractal islands where it yields $\sigma_{x}=2+x^{1 / 1.7}\left(\sigma_{1}=3\right)$, with $x$ being the island size in atoms; the constant of 2 accounts for atoms diffusing to sites adjacent to the island perimeter [59]. As can be seen in Fig. 1(a) this approximation is inconsistent since it yields a significantly steeper slope in the Arrhenius representation of the island densities than that expected from Eq. (8) ( $\chi$ is by $30 \%$ too large compared to the the expected value of $\frac{1}{3}$, see Table 1). This inconsistency comes from disregarding the diffusion field towards the islands which is driven by the gradient in the monomer concentration. An alternative approximation is to assume constant capture rates $[10,60]$. This assumption is based on the fact that more sophisticated approximations for the capture rates show that these do not change very much in the coverage range close to saturation (see Fig. 2(a) of Ref. [10]). From Fig. 1 it is seen that for example $\sigma_{1}=3$ and $\sigma_{x}=7$ work very well (compare Table 1). However, constant capture rates become less suited outside the saturation regime where the change of $\sigma_{x}$ with coverage has to be accounted for.

A more sophisticated approach for the capture rates is obtained from solving the 2D diffusion equation in the lattice approximation, where islands are assumed to be placed on a periodic lattice $[10,60]$. This yields an analytic expression for $\sigma_{x}$ that depends on the fraction of the surface covered by

Fig. 1. (a) Results from integrating rate equations (5) and (6) within various approximations for the capture numbers $\sigma_{1}$ and $\sigma_{x}$ up to $\Theta=0.12 \mathrm{ML}$. They are compared to the scaling law equation (8) $\left(\eta=0.25, \chi=\frac{1}{3}\right)$ and to the self-consistent solutions for compact islands reported in Ref. [12]. Fractal sigma stands for the geometric concept applied to fractal islands. In all cases coalescence is neglected. (b) Effect of direct impingement onto islands and monomers, as well as onto their direct neighbor sites and their next nearest neighbor sites. Also the influence of the coalescence term is shown (calculation within lattice approximation). (c) Approximation for $\sigma_{1}$ with respect to a constant value of $\sigma_{1}=3$. All comparisons are done for the case of compact islands. 
a)

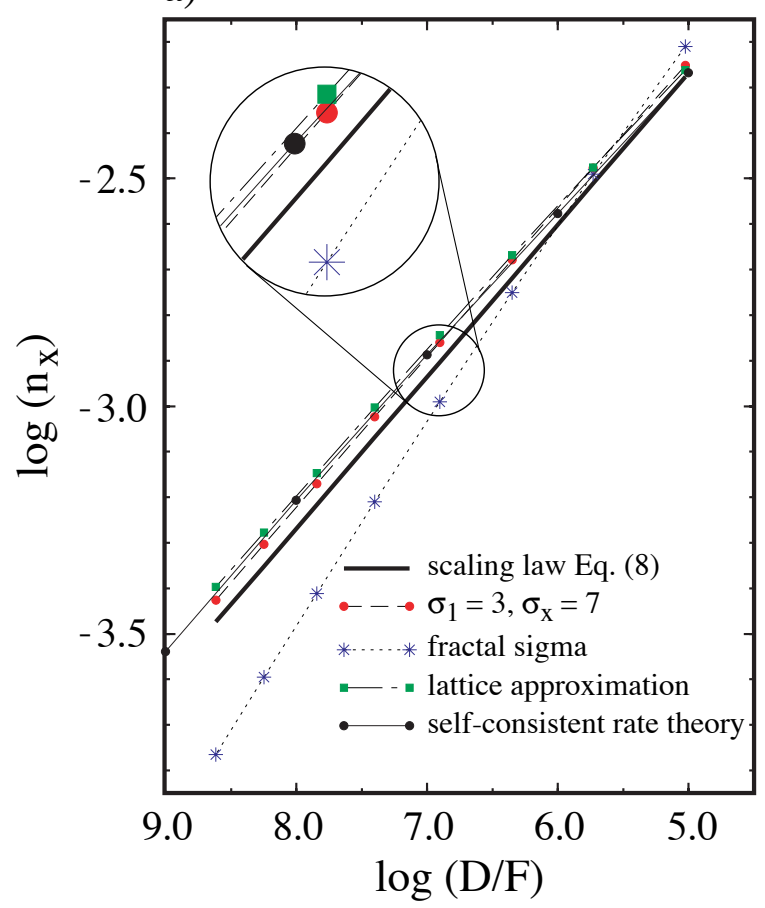

c)

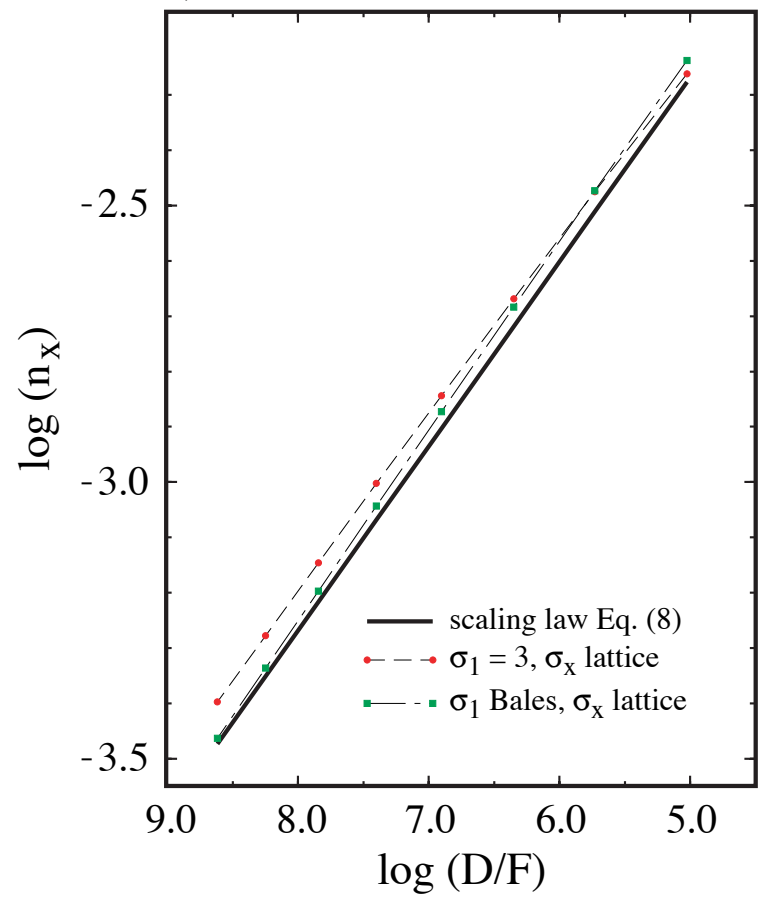

b)

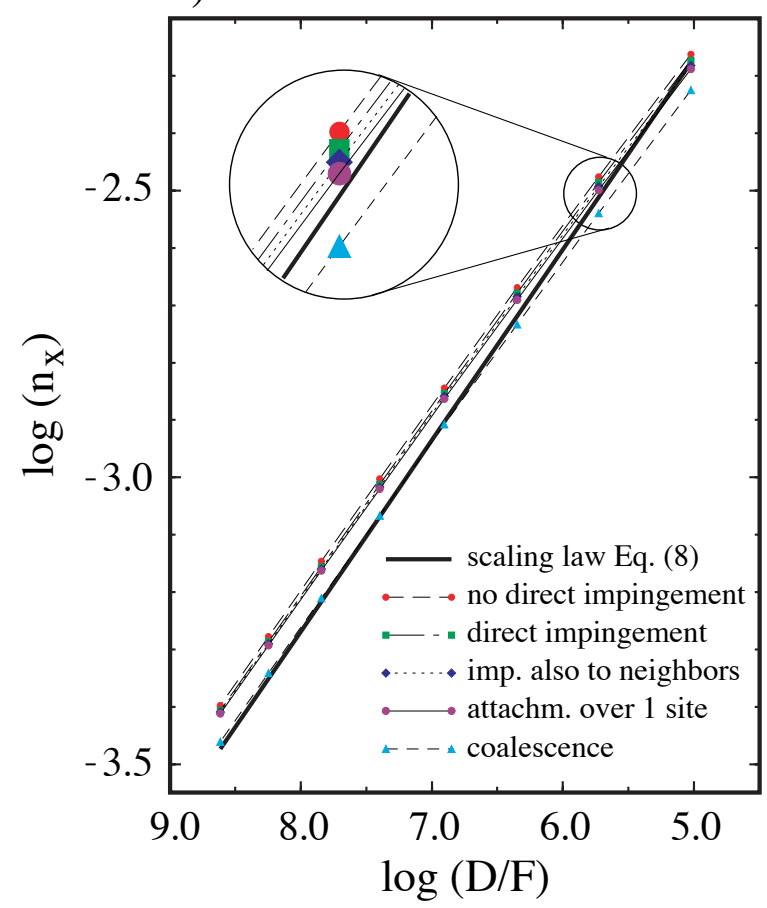


Table 1

Prefactors $\eta$ and slopes $\chi$ obtained from linear regressions to the data shown in Fig. 1

\begin{tabular}{llll}
\hline Approximation & $\eta(\Theta=0.12, i=1)$ & $\chi$ & $3 * \chi$ \\
\hline Eq. (8) & 0.2500 & 0.3333 & 1.0000 \\
$\sigma_{1}=3, \sigma_{x}=7$ & $0.249 \pm 0.004$ & $0.327 \pm 0.001$ & $0.981 \pm 0.003$ \\
Fractal sigma & $1.00 \pm 0.07$ & $0.434 \pm 0.004$ & $1.30 \pm 0.01$ \\
Lattice & $0.218 \pm 0.006$ & $0.317 \pm 0.002$ & $0.951 \pm 0.006$ \\
Uniform depletion [12] & $0.21 \pm 0.01$ & $0.317 \pm 0.002$ & $0.951 \pm 0.006$ \\
$\sigma_{1}$ from [12], $\sigma_{x}$ lattice & $0.304 \pm 0.004$ & $0.3416 \pm 0.0008$ & $1.025 \pm 0.002$ \\
\hline
\end{tabular}

stable islands $Z=\Theta-n_{1}$ (for $i=1$, with $\Theta=F t$ being the total coverage) [10]:

$$
\begin{aligned}
\sigma_{x} & =\frac{4 \pi(1-Z)}{\ln (1 / Z)-(3-Z)(1-Z) / 2}, \\
\sigma_{1} & =4 \pi\left(1-n_{1}\right) \frac{n_{x}}{n_{1}} \frac{1}{\ln (1 / Z)-(3-Z)(1-Z) / 2} .
\end{aligned}
$$

Expression (9b) for the capture rate of monomers can be derived from Eq. (26) of Ref. [10]. For monomers, a constant value of $\sigma_{1}=3$ can be used for simplicity since $\sigma_{1}$ varies only slowly with coverage. The result from numerically integrating the rate equation within this approximation is seen to yield a slope which is too shallow by $5 \%$. Absolute numbers for $n_{x}$ come out correctly to within $10 \%$. This absolute precision is sufficient as often unknown details like attachment towards islands can change absolute numbers within such a range (see Fig. 1(b)).

The expression for $\sigma_{x}$ obtained from the uniform depletion approximation, similar to Eq. 9(a), only depends on the coverage $Z$ contained in stable islands [10,61]:

$$
\begin{aligned}
& \sigma_{x}=2 \pi \sqrt{\frac{\sigma_{x} Z}{\pi}} K_{1}\left(\sqrt{\frac{\sigma_{x} Z}{\pi}}\right) / K_{0}\left(\sqrt{\frac{\sigma_{x} Z}{\pi}}\right), \\
& \sigma_{1}=2 \pi \sqrt{\frac{\sigma_{x} n_{x}}{\pi}} K_{1}\left(\sqrt{\frac{\sigma_{x} n_{x}}{\pi}}\right) / K_{0}\left(\sqrt{\frac{\sigma_{x} n_{x}}{\pi}}\right),
\end{aligned}
$$

where $K_{1}$ and $K_{0}$ are the modified Bessel functions. Since $\sigma_{x}$ appears in the argument, this approximation is numerically more demanding. It involves solution of the implicit equations (10), to be performed in the course of numerical integration of Eqs. (5) and (6) since it also contains the densities $n_{1}$ (via $Z$ ) and $n_{x}$. Note that circular islands have been assumed for deriving Eqs. (10).

The comparison in Fig. 1(a) shows that lattice and self-consistent uniform depletion approximation, as well as the case of constant capture rates, all lie rather close to each other. This makes all of them suited for comparison to experimental island densities. The precision of absolute numbers is certainly sufficient, however, when analyzing slopes one has to be aware of systematic errors up to 5\%. The approximation that leads to considerable systematic errors is the geometrical concept.

The effects of island formation by direct impingement onto monomers and of the disappearance of monomers due to impingement onto (and subsequent descent from) stable islands are addressed in Fig. 1(b). It becomes evident from inspection of Fig. 1(b) that these effects are rather small at $0.12 \mathrm{ML}$ and in the scaling regime of $10^{5}<D / F<10^{9}$ addressed here. Below that regime, however, impingement 
onto and diffusion towards islands and monomers become quite important as will be seen in Section 3.4. We therefore discuss briefly how these effects can be accounted for by rate theory. Impingement onto monomers and islands are incorporated in Eqs. (5) and (6) through the terms with $\kappa_{1}$ accounting for the first and $\kappa_{x}$ for the latter. If we want to allow for direct impingement only, then $\kappa_{1}=\kappa_{x}=1\left(\kappa_{x}=1\right.$ implies that the impingement rate is proportional to the average size of stable islands times their density, i.e. the coverage $Z=F t-n_{1}$ contained in stable islands). It is very likely that arrival onto sites neighboring a monomer also leads to island formation. This requires $\kappa_{1}=5$ (7) on a square (hexagonal) lattice, and the term containing $\kappa_{x}$ becomes $-F A_{x} n_{x}$, with $A_{x}$ being the attachment area of an average island: $A_{x}=\pi(\sqrt{\operatorname{size} / \pi}+1)^{2}$ with size $=\left(\Theta-n_{1}\right) / n_{x}$. Attachment of monomers to islands and other monomers over one nearest neighbor distance may have a smaller barrier than regular terrace diffusion (neighbor driven mobility, see Section 3.4). Incorporation into rate equations leads to $\kappa_{1}=13$ (19) on a square (hexagonal) lattice, and $A_{x}=\pi(\sqrt{\operatorname{size} / \pi}+2)^{2}$.

As expected, impingement onto and attachment to islands and monomers lead to a decrease of island densities since the overall monomer density becomes reduced. These effects become more important when the intrinsic mobility becomes smaller, i.e. at smaller $D / F$. The quantitative effects are, however, rather small. The results incorporating impingement and attachment are hardly discernible from results that completely neglect direct impingement $\left(\kappa_{1}=\kappa_{x}=0\right)$. It is therefore not important to account for these effects in the range of $D / F$ addressed here. We will see in Section 3.4, however, that attachment to islands and monomers becomes important at lower temperatures and/or larger deposition rates.

As can be seen from Eq. 9(b) the expression given by the lattice approximation for the capture rate of monomers is more complicated than that of $\sigma_{x}$. It contains the densities $n_{1}$ and $n_{x}$ which renders numerical integration of the rate equations more costly. Approximation of $\sigma_{1}$ by a constant value is therefore convenient. We show in Fig. 1(c) that this approximation leads to a 5\% smaller slope than that from the classical scaling result, Eq. (8). The expression proposed by Bales and Chrzan for $\sigma_{1}$ increases this slope and brings the lattice approximation closer to Eq. (8) (cf. Table 1).

In this section, we have discussed the results from mean-field nucleation theory for the most relevant case for the experiments to be described in this review, i.e. metal epitaxy at low temperatures, where $i=1$ and for the material condensing into 2D islands on isotropic substrates. For that case, mean-field nucleation theory yields a scaling law for $n_{x}$ with $D / F$ expressed in Eq. (8). This law is valid for compact islands and in the coverage regime of saturation. For comparison to data obtained at smaller coverage, rate equations can be integrated, for example within lattice or uniform depletion approximation, yielding results very close to Eq. (8). The systematic errors of these approximations for the scaling exponent are in the range of 5\%. The uniform depletion approximation represents a lower limit, and the lattice approximation an upper limit to the real but unknown solution to the capture rates.

Rate theory gives a very good feeling for the relative importance of the various processes involved in nucleation and it permits the prediction of average quantities with rather good precision. Nevertheless, there are two remaining issues that are not satisfactorily described. Both are connected to the meanfield approach that disregards correlations between islands. The mean-field approach assumes that the mean density of monomers sufficiently far away from the island takes on a constant value, no matter what the local environment of the island looks like. The first consequence is that, although average island sizes are predicted correctly as are island densities, the island size distributions are far from being realistic [12,62]. As a second consequence, coalescence cannot be described very accurately (see Fig. 3(a)). For example, what happens to the capture rates when two islands coalesce? Both issues are 
a)

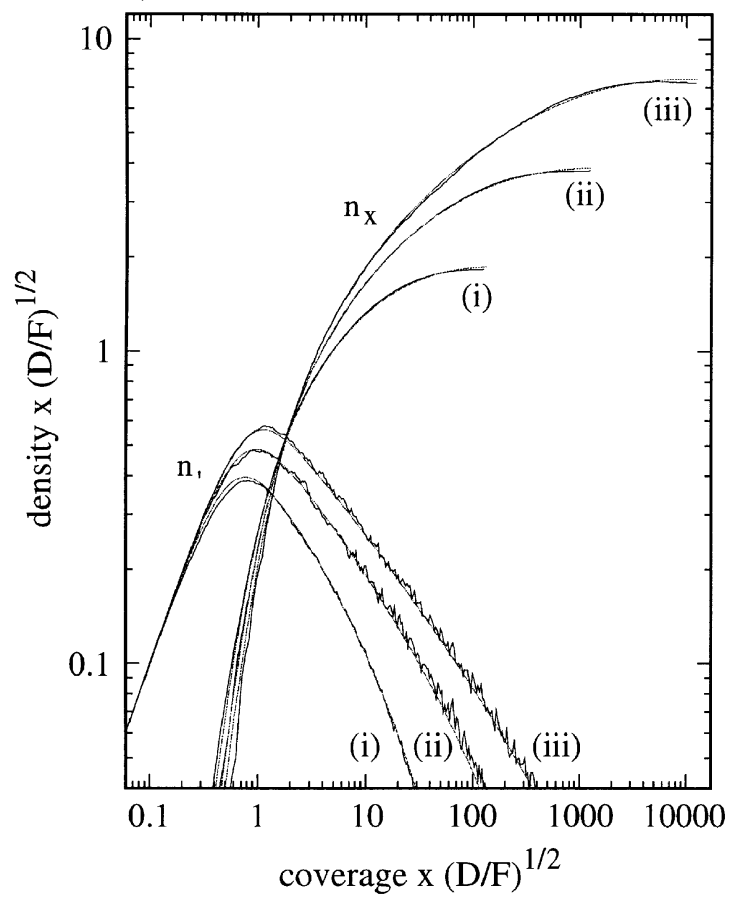

b)

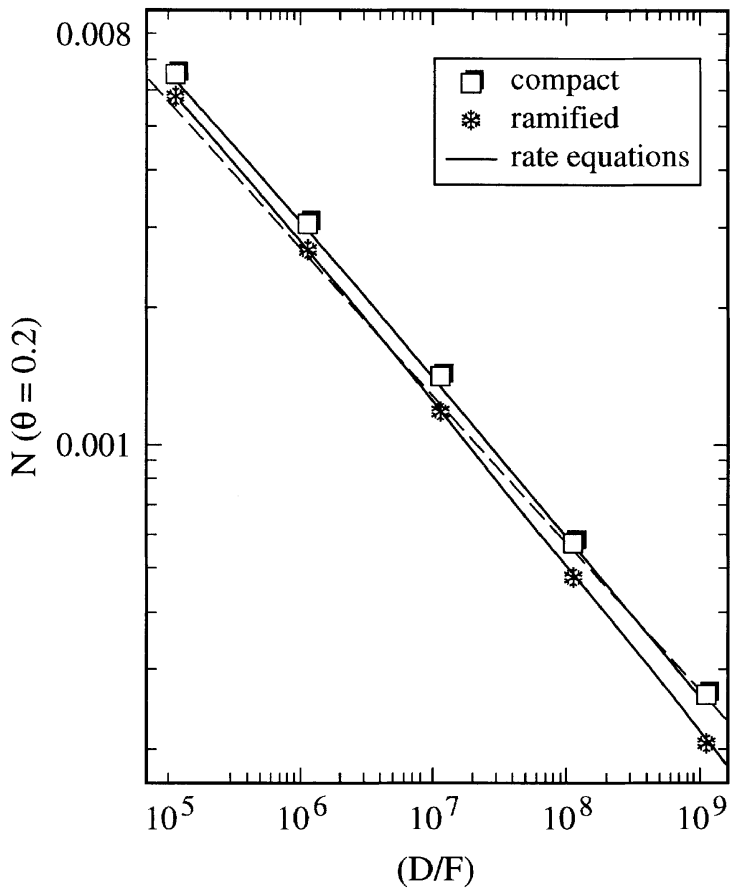

Fig. 2. Comparison of rate theory to KMC simulations. (a) Monomer density $n_{1}(\Theta)$ and density of stable islands $n_{x}(\Theta)$ vs. coverage for ramified islands at (i) $D / F=10^{5}$, (ii) $D / F=10^{7}$, and (iii) $D / F=10^{9}$. The solid lines are measured from KMC simulations performed on a square lattice, and the dotted lines are numerical solutions of the rate equations. (b) The same comparison for $n_{x}(D / F)$ both for compact and ramified islands. KMC was performed on a square lattice (the dashed line shows the scaling law equation (8)) (from Ref. [12]). The agreement between self-consistent mean-field theory and MonteCarlo simulation is striking in both cases.

very difficult to address and reach the limits of a mean-field theory. This implies that present rate theory yields reliable results only for $\Theta \leq \Theta_{\text {sat }}$ and for average quantities. However, the precision of these results is striking, as demonstrated by Fig. 2 which compares self-consistent rate theory to kinetic Monte-Carlo (KMC) simulations. Apart from this agreement, Fig. 2(b) also shows that the scaling exponent $\chi$ appearing in Eq. (8) slightly varies for different island shapes. We will quantify this variation below in a more detailed discussion of $\mathrm{KMC}$ results. Note the precision to which rate theory can be adjusted to account for this effect.

\subsection{Kinetic Monte-Carlo simulations}

In KMC simulations, the randomness of the spatial distribution of islands, as well as island-island interactions via the diffusion field are implicitly included. Various island shapes can also be incorporated. These simulations therefore serve as a rather valid test for nucleation theory (Fig. 2). They can also be employed to test ideas on mechanisms underlying pattern formation as well as to study crossover scaling, for example observed for nucleation in the presence of a certain density of defects. For both cases we will quote examples further below. Due to the increasing importance of 
KMC, manifested in its frequent appearance in recent literature on epitaxial growth [63], a brief description of this technique is appropriate. This will be followed by a presentation of the central results from KMC simulations for scaling of island densities as a function of island shape. As coalescence is captured well by these simulations they allow light to be shed on scaling of island densities at higher coverages. This yields particularly valuable information for the interpretation of experiments performed with diffraction techniques that generally require a minimum coverage above the typical saturation value.

In a typical kinetic Monte-Carlo program ${ }^{6}$ one has a list of displacement processes allowed for the various atoms present on the surface. In this list, each atom can appear with several processes, e.g. with diffusion towards an island into one particular direction and regular terrace diffusion into all other directions. Likewise an atom situated at a step can diffuse along the step into two opposite directions. On a square lattice, the third allowed movement for such an atom would be its dissociation from the edge towards the free terrace. The process list also includes simple deposition of a new atom. There is a specific rate linked to each type of process. These rates are jump rates, $\nu_{n}=\nu_{0} \exp \left(-E_{n} / k T\right)$ for diffusion, and $\nu_{\text {dep }}=F \times$ (system-size) for deposition of a new atom. To each process we can associate a vector with a length corresponding to its rate. Now a pointer, driven by a random number generator, selects one vector among a field where all the vectors belonging to all possible processes are lined up. That way, the probability for selecting a process is correctly made proportional to its frequency of appearance times its rate. After effecting the move of the selected atom in the selected direction, the process list is updated since the coordination of the atom itself, and of its neighbors, might have been affected by the move. Again a new random number is generated to select the next process from the list, and this procedure is repeated many times. One typically has parameter files for $T, F, \nu_{0}$ and $E_{n}$ as well as the system size, which is periodically repeated. In principle, this is a simple program capturing the physical situation very well. One has to be aware of a few drawbacks, however. The random number generators, which can be of poor quality [66], have a limited depth reducing the largest ratio of the slowest to the fastest rate which can be simulated to typically $10^{9}$. In addition, one has to watch out for finite size effects due to limited system size [63]. Finally, for significant statistics, one might need a considerable amount of runs which is a question of CPU time. ${ }^{7}$

\subsubsection{Scaling of island size distributions}

The issue of island size distributions obtained from such KMC simulations has been treated extensively in recent literature. It is predicted from scaling theories that island size distributions, when scaled properly, all fall onto common curves that only depend on the critical cluster size $i$ [67-74]. Experimental verification of this scaling was demonstrated in STM measurements for the system $\mathrm{Fe} / \mathrm{Fe}(100)$, where the agreement of scaled size distributions with theoretical curves was used to identify the stable cluster size [75].

Size distributions generally bear valuable information. They serve as fingerprints for the critical cluster size [75-77]. This holds down to the case of $i=0$, characterized by a monotonically decreasing size distribution [78]. This case is realized for instance when exchange renders incoming atoms immobile at their point of impact (in that sense a monomer is considered as a stable nucleus and hence

\footnotetext{
${ }^{6}$ The description given here refers to a program written by J. Jacobsen [64], see also Ref. [65].

${ }^{7}$ In the KMC code developed by Jacobsen, $10^{6}$ moves take 2 min CPU time on a DEC ALPHA 3000-900 workstation. A deposition experiment of $0.1 \mathrm{ML}$ on a lattice of $500 \times 500$ sites can involve up to $10^{9}$ moves and requires 100 Mbyte memory.
} 
$i=0$ ) [79] or if impurities or other special sites on the surface act as pinning sites. A similar behavior is observed when most of the mobility takes place after deposition [77]. The latter case generally characterizes deposition at very low temperatures, therefore it is of general interest and will be treated in more detail below (see Section 3.4.). Since size distributions are strongly affected by dimer and cluster mobility [80-82] they can also serve to exclude this kind of mobility $[81,83]$.

\subsubsection{Coalescence and island shape}

As coverage increases, correlations between adjacent islands become increasingly important in determining their growth rate up to coalescence. Mean-field theories do not include these effects and so they necessarily have difficulties in correctly describing island coalescence. ${ }^{8}$ The actual onset of coalescence becomes evident when comparing the curves obtained from self-consistent rate theory and a KMC simulation which neglects coalescence to the KMC result taking coalescence into account (see Fig. 3(a)). The curves become discernible at $\sim 0.1 \mathrm{ML}$, but coalescence effects remain small until

a)

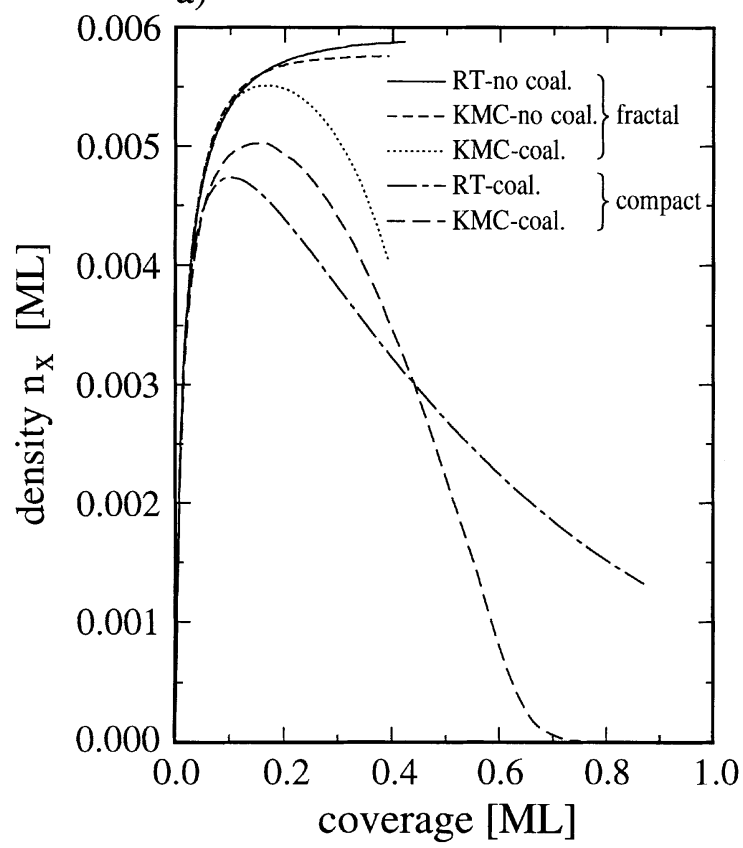

b)

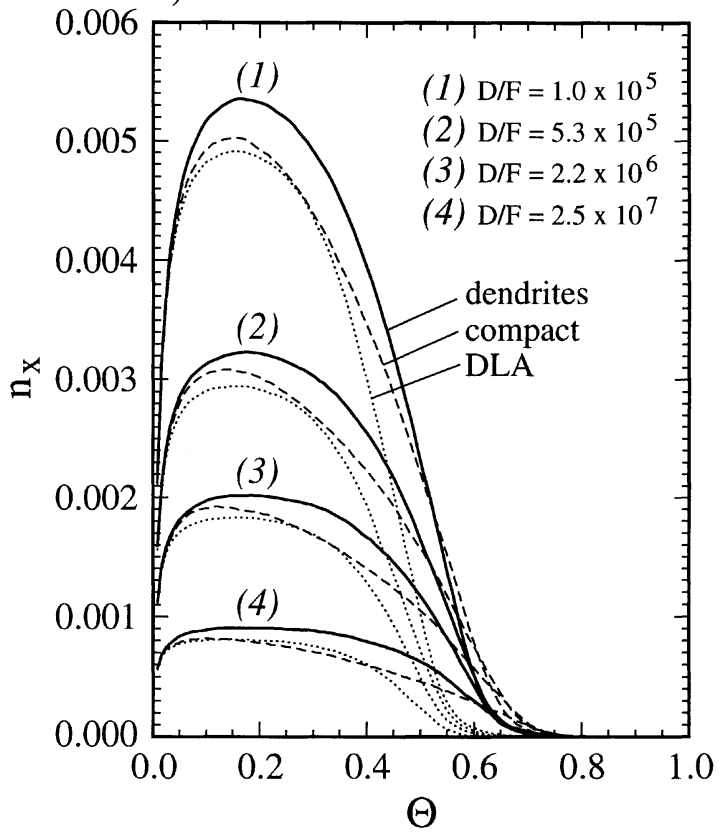

Fig. 3. Coalescence investigated with KMC simulations and rate theory for $D / F=10^{5}$. (a) Situation for fractal islands on a square lattice: Coalescence begins to affect island densities at $\Theta \geq 0.2 \mathrm{ML}$. Compact islands on a hexagonal lattice: The coalescence term of mean-field theory is seen to overestimate coalescence effects at early times, whereas it does not drop the island density to 0 at $\Theta=1 \mathrm{ML}$. (b) KMC simulations for three island shapes that can be realized on hexagonal lattices; an abrupt drop of island densities for both cases of ramified islands contrasts earlier coalescence, extended over a larger coverage regime for compact islands (from Ref. [85]).

\footnotetext{
${ }^{8}$ There are attempts to include island correlations into mean-field theory in order to provide a realistic description of coalescence [84].
} 
0.2 ML. The standard coalescence term from mean-field theory is seen to be unsatisfactory as it overestimates coalescence at low coverages; and still it does not sufficiently drop island densities at higher coverages [10].

Islands formed on square lattices are typically compact [86,87]. This is due to the fact that edge atoms are laterally only one-fold coordinated and hence move with a barrier comparable to terrace diffusion [88]. Complete relaxation into perfect squares involves diffusion around corners which has a higher barrier, however. There are a few exceptions to this picture of islands generally being compact on square lattices. A trivial one is $\mathrm{Ag} / \mathrm{Ni}\left(\begin{array}{lll}1 & 0 & 0\end{array}\right)$, where considerable misfit drives the adatoms to form a hexagonal overlayer which is characterized by less edge mobility [89]. A less trivial example is $\mathrm{Cu} / \mathrm{Ni}\left(\begin{array}{lll}1 & 0 & 0\end{array}\right)$ where the formation of irregular island shapes is thought to be driven by better strain relief at the edges [90]. Island shapes on hexagonal surfaces will be discussed in detail in Section 5. Here, it suffices to mention that at low temperatures predominantly dendrites are observed which obey preferred growth directions causing trigonal symmetry. Classical DLA islands that would result from a simple hit and stick mechanism have never been observed. However, at higher temperatures (or low flux), branches grow in random directions [17,91]. Although these islands have thicker branches due to edge diffusion [92], they closely resemble DLA clusters, rending this species more than a simple textbook example.

Coalescence for these three kinds of islands on hexagonal lattices is displayed in Fig. 3(b) showing island densities up to $1 \mathrm{ML}$ [85]. Earliest coalescence is observed for compact islands. At first glance, this might be surprising to the reader since compact islands percolate latest (see Fig. 3(b)). To rationalize early coalescence for compact islands we imagine the situation just before coalescence when islands are rather close to each other. For compact islands the narrow spacing between adjacent islands still has a sufficient supply of atoms provided via edge diffusion from parts of the edge having access to the diffusion field of the free terrace. For limited edge diffusion, on the other hand, this supply is considerably slowed down. Since then islands grow fastest in the directions of the largest adatom supply from the terrace they tend to avoid each other. This shadowing explains the delay of coalescence observed in Fig. 3(b) for the two types of ramified islands. For these island shapes coalescence sets in very suddenly, leading to a steep decrease of the island density. This reaches its half maximum at 0.43 ML for DLA, and somewhat later at $0.48 \mathrm{ML}$ for dendritic growth. The slightly later coalescence of dendritic islands is related to their trigonal symmetry, implying that branches have to grow closer towards the center of neighboring islands in order to coalesce.

\subsubsection{Scaling for coverages below and above saturation}

It is important to test the validity or to determine necessary modifications to the scaling law expressed in Eq. (8) in the high coverage limit, as most of the experimental techniques working in reciprocal space require a minimum coverage above saturation. For instance, the side bands in diffraction scans obtained with low energy electron diffraction (LEED) clearly emerge only at and above 0.3 ML [93-95]. He diffraction experiments were performed at coverages as high as 0.5 ML [96]. Island densities vs. $\log (D / F)$ are represented in Fig. 4(a) for dendritic islands and various coverages ranging from 0.05 to $0.55 \mathrm{ML}$. First, it becomes obvious that the island densities obtained for 0.10 to $0.30 \mathrm{ML}$ fall into a very narrow regime. In addition, in this coverage range the island densities can be approximated fairly well by straight lines. In contrast, the densities at 0.05 and those for $\Theta>0.30 \mathrm{ML}$ lie significantly below those characterizing saturation. Furthermore they show considerable deviations from straight lines, i.e. they escape from scaling by a single exponent (see $\Theta \geq 0.50 \mathrm{ML}$ ). 
a)

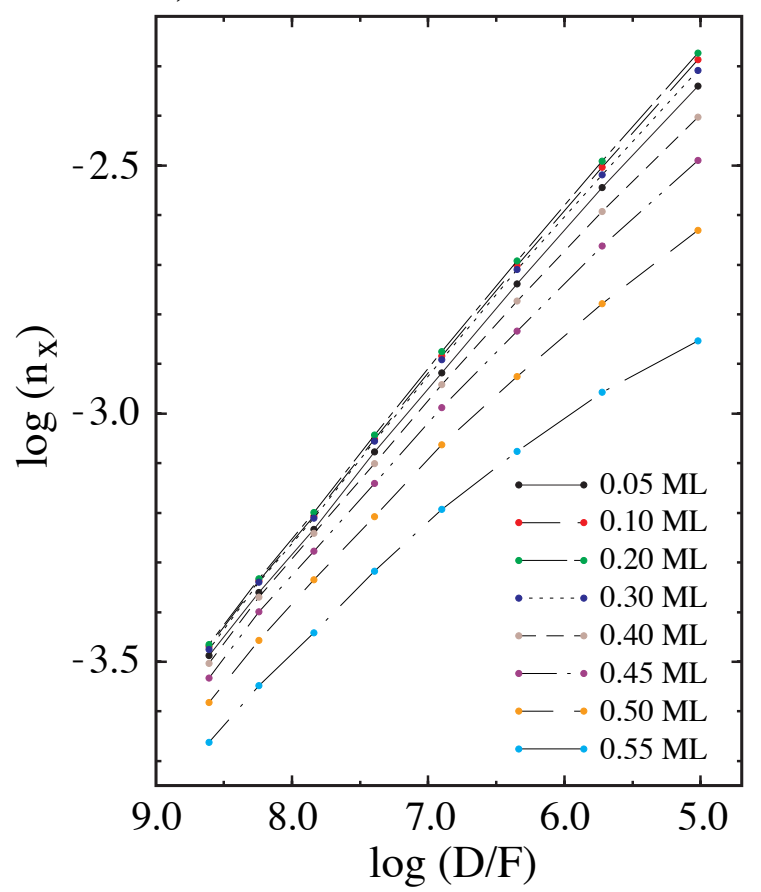

b)

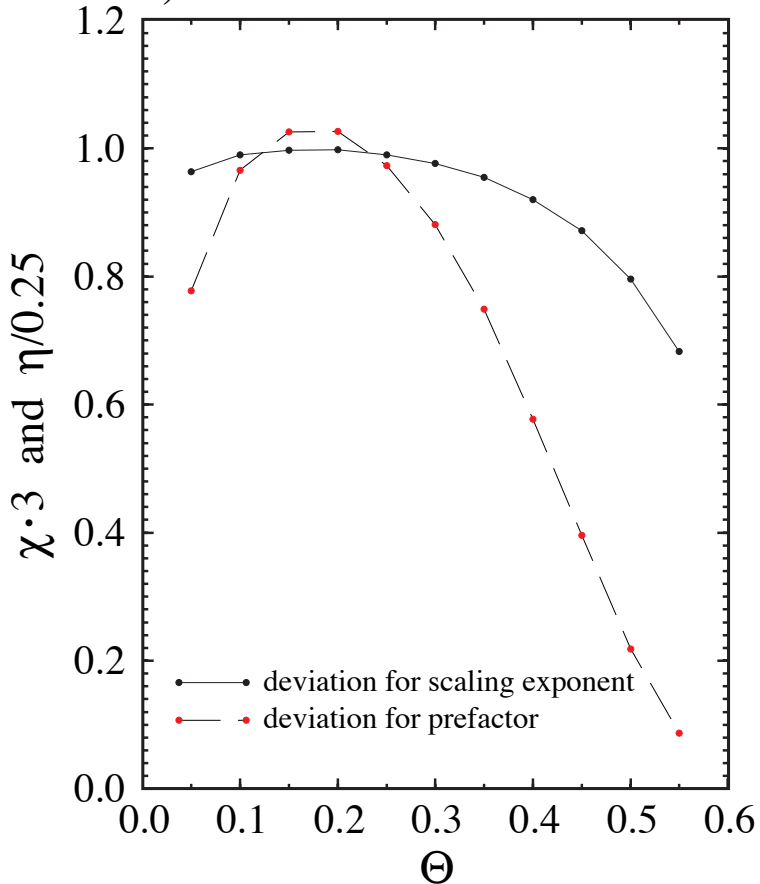

Fig. 4. (a) Scaling of island densities for different coverages as obtained from Monte-Carlo simulations for dendritic islands on a hexagonal lattice. (b) Deviation from the scaling law of mean-field nucleation theory expressed in Eq. (8) for the exponent $\chi$ and the prefactor $\eta$ due to the results represented in (a) (from Ref. [85]).

From the data represented in Fig. 4(a), one can monitor the variation of the scaling exponent $\chi(\Theta)$ and the prefactor $\eta(\Theta)$ and thus empirically extend the applicability of Eq. (8) to a large coverage regime around saturation and also to the various island shapes. For that purpose, linear regressions to the KMC results are analyzed in terms of deviations from the exponent of $\chi=\frac{1}{3}$ and the prefactor $\eta=0.25$ in Fig. 4(b). For the saturation regime of $0.10 \leq \Theta \leq 0.30 \mathrm{ML}$, the scaling exponent stays constant within $2 \%$. Its value is precisely $\frac{1}{3}$ for our example of dendritic islands on hexagonal surfaces. This is surprising in view of the exponent for DLA islands on quadratic lattices being slightly higher $[12,56,72,85]$. From the small variation of $\chi$ in the saturation regime it can be concluded that reliable barriers can be extracted between 0.1 and $0.3 \mathrm{ML}$. As a matter of fact experimental variations in total coverage within a temperature series have only very small impact on the result for $E_{\mathrm{m}}$. The island shape dependent character of coalescence is reflected in the variations of $\chi$ and $\eta$. They begin to change earlier for compact islands and later but more abruptly for both types of ramified islands [85]. Results for $v_{0}$ generally require knowledge on the particular island shape as they rely on absolute number densities. The strong variation of $\eta$ with coverage expresses the sensitivity of $v_{0}$ to these details. The small variation in $\chi$ for coverages up to $0.4 \mathrm{ML}$ is encouraging for LEED and He-diffraction studies. Nevertheless, one should be aware of the breakdown of scaling for higher coverage. This breakdown is quantified by the KMC-results discussed here which establishes analysis of experimental data obtained at coverage below and above the scaling regime. 
In summary, we have given a brief introduction into nucleation theory with special emphasis on the link between surface diffusion and nucleation island densities. We reported on the progress achieved in recent literature in developing a self-consistent mean-field theory, which can be adjusted (by a single fitting parameter) to correctly describe compact, as well as ramified islands. Comparison of several classical expressions for the rate coefficients shows that most of them yield sufficient precision. Rate equations can be numerically integrated within these assumptions in a straightforward way. The scaling law linking nucleation island densities at saturation to the ratio of diffusivity and flux is, strictly speaking, only an approximation, as island densities do lie on a slightly curved line in an Arrhenius representation. This curvature is due to a weak dependence of capture rates on $D / F$, an effect which is more pronounced for DLA islands. The approximation made in analyzing island densities by Eq. (8) is, however, extremely good as deviations from linearity of $\log \left(n_{x}\right)$ vs. $1 / T$ are below $2 \%$ which is typically comparable to the experimental error margin. The effect of island shape and coverage on the scaling exponent in Eq. (8) can lead to deviations from the classical value $\eta=1 / 3$ of up to $5 \%$. Taking these effects into account, the straightforward analysis of island densities by Eq. (8) evolves as the most direct access to diffusion parameters from island densities. It should be noted, however, that this approach is only valid for $i=1$ and $D / F \geq 10^{5}$, the latter restriction is due to post-deposition mobility as will be elucidated further below. It turns out that self-consistent rate theory and KMC simulations yield fully consistent results for various island symmetries. It is therefore not relevant for the resulting diffusion parameters whether the analysis of island densities is performed with one or the other. Both methods allow extension to coverages below saturation. As island correlations are not included in rate theory it fails to correctly describe coalescence and island size distributions. For these issues, KMC simulations are more suited, and permit investigation of density scaling also above saturation.

\section{Microscopic view of nucleation}

For many years, FIM experiments have allowed very detailed observations providing quantitative information on the various diffusion processes which contribute to the epitaxial growth of metals [7,9799]. Until recently, however, much less has been known about the interplay of these processes during thin film growth and how they determine the final island and film morphology. As mentioned above, nucleation theory, describing what should happen in the course of a deposition experiment, has existed since quite some time. Numerous experiments to test these theories have been carried out using electron microscopy (EM) [11]. For metal on metal condensation a direct comparison to theory was hampered by the fact that the EM experiments were performed with systems and at temperatures where only larger clusters are stable. A substantial number of cluster binding energies were therefore involved as free parameters. A breakthrough was achieved with the application of STM to variable (low) surface temperature $[100,101]$.

Microscopic information on the outcome of a deposition experiment as a function of temperature allows one to draw conclusions on thermally activated processes and to trace back the way they have contributed to the resulting morphology. The present section addresses these recent experimental observations. This is done by illuminating the role they play as experimental tests of nucleation and scaling theories and by establishing how quantitative information on surface diffusion can be extracted with a precision so far only obtained by FIM. These STM experiments are not restricted to elements of large mechanical strength required of FIM. Furthermore, the application of STM to substrates which are isotropically strained provides valuable information on the role of strain in surface diffusion. 


\subsection{From nucleation to growth}

\subsubsection{Growth scenario for $i=1$}

The simplest growth scenario described at the beginning of Section 2 has monomers as the only mobile species and dimers represent stable nuclei. This scenario represented a mere text book example of nucleation theory, until recently when it could be realized experimentally with Ag nucleation on a $\mathrm{Pt}\left(\begin{array}{lll}1 & 1 & 1\end{array}\right)$ surface held at $75 \mathrm{~K}$ [59]. Four STM images taken at the deposition temperature of $75 \mathrm{~K}$ are shown in Fig. 5. The very initial stages of nucleation are depicted in Figs. 5(a) and (b). The islands,

a) $\Theta=0.0024 \mathrm{ML}$

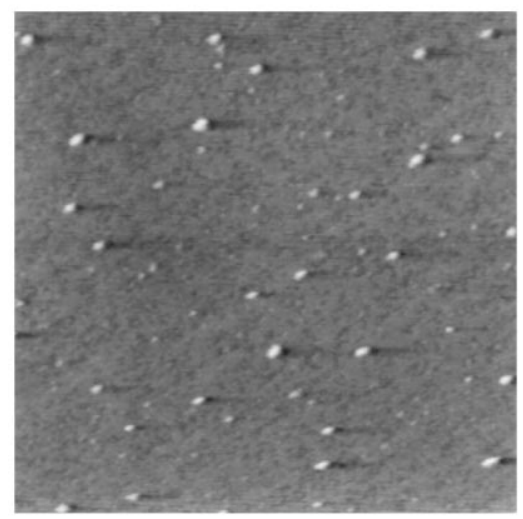

$\bar{n}=2.4 \pm 0.4$

c) $\Theta=0.03 \mathrm{ML}$

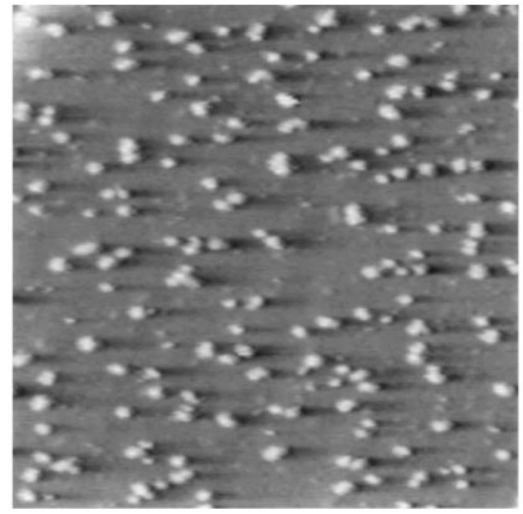

$$
\overline{\mathrm{n}}=6.4 \pm 1.1
$$

b) $\Theta=0.006 \mathrm{ML}$

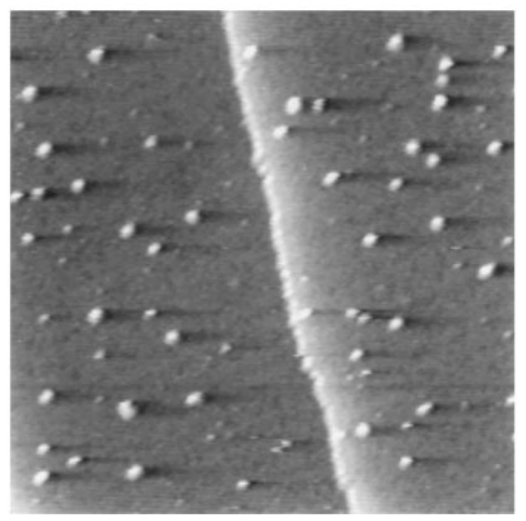

$\overline{\mathrm{n}}=2.6 \pm 0.5$

d) $\Theta=0.06 \mathrm{ML}$

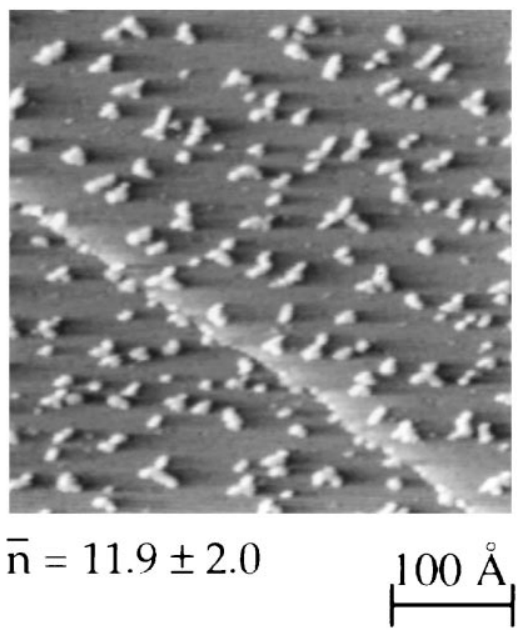

Fig. 5. STM images showing the transition from the very early nucleation phase to island growth on hand of the evolution of the island density and size with increasing coverage for $\mathrm{Ag}$ deposition $\left(F=1.1 \times 10^{-3} \mathrm{ML} / \mathrm{s}\right)$ onto $\mathrm{Pt}(111)$ at $75 \mathrm{~K}$. Coverages and mean island sizes are indicated (from Ref. [59]). 
detectable as bright spots, predominantly constitute dimers and trimers. This is inferred from the mean island size, calculated from the coverage divided by the density, yielding $2.4 \pm 0.4$ atoms and $2.6 \pm 0.5$ atoms, for Figs. 5(a) and (b), respectively. This is exactly the value expected from nucleation involving dimers as stable nuclei. During the course of deposition, dimers form until they reach a density similar to that of the diffusing monomers. At this stage, the probability for monomers meeting each other and creating new nuclei (dimers), and that for attaching to existing dimers (resulting in trimers) become equal. The result is a mixture of mostly dimers, trimers and a few tetramers, giving rise to the mean island size of about three atoms. The fact that the mean size does not change from Fig. 5(a) to (b), despite an increase in the coverage by about a factor of 3, is characteristic of the so-called pure nucleation regime, where additional deposition predominantly results in the formation of new nuclei. Increasing the coverage further by a factor of 5 leads to the transition from nucleation to growth. In Fig. 5(c), the density is increased by a factor of 2 over that of Fig. 5(b). This is accompanied by a considerable increase in the average island size to $6 \pm 1$ atoms. Further increase of the coverage by a factor of 2 leads to exclusive island growth (12 2 atoms per island in Fig. 5(d)). Thus the last figure shows the pure growth or steady state regime, where the island density has saturated. The island density is now such that each arriving film atom has sufficient mobility to reach an island with a higher probability than that for hitting a second mobile adatom and create a new nucleus.

Note that Figs. 5(c) and (d) also contain information on the island shape instability towards ramification. Whereas the islands in Fig. 5(c), containing on the average six atoms, are compact, they begin to ramify into Y's at a size of 12 atoms (Fig. 5(d)). These Y's are all oriented the same way (see also Figs. 6(a) and (b)). This trigonal symmetry is reflected for larger islands by their triangular envelope (Fig. 6(c)). The atomic process giving rise to this particular shape will be addressed in detail in Section 5 on aggregation, and we presently focus on island densities.

The set of STM images represented in Fig. 5 completely characterizes nucleation of $\mathrm{Ag} / \mathrm{Pt}\left(\begin{array}{lll}1 & 1 & 1\end{array}\right)$ at $75 \mathrm{~K}$, as it yields the saturation island density (Fig. 5(d)) as well as the stable nucleus size (Figs. 5(a) and (b)) at that temperature.

\subsubsection{Analysis of island densities}

A quantitative study of surface diffusion requires knowledge of the variation of the island density as a function of substrate temperature, ideally obtained at $i=1$. One of the first systematic STM studies of this kind was reported by Stroscio and Pierce for Fe(100) homoepitaxy [86]. (There is an earlier example for $\mathrm{Si} / \mathrm{Si}\left(\begin{array}{ll}10 & 0\end{array}\right)$ by Mo et al. [76]. We will, however, focus on examples obtained for metals.) The nucleation of Fe was studied above room temperature and island densities were determined by means of STM. The densities were analyzed in assuming that dimers were stable. Despite the relatively high temperature, dimers turned indeed out to be stable, ${ }^{9}$ as verified using island size distributions in a later publication [75]. On close-packed surfaces, activation energies, such as the dimer dissociation barrier, are generally small requiring low temperatures to guarantee $i=1$. For our example of $\mathrm{Ag} / \mathrm{Pt}\left(\begin{array}{lll}1 & 1 & 1\end{array}\right)$, it was verified that dimers constitute stable nuclei up to $110 \mathrm{~K}$, through observation of the onset temperature for Ostwald ripening [22].

Three STM images reproduced in Figs. 6(a)-(c) show the strong variation of the saturation island density with temperature. As outlined above, these densities directly reflect the adatom mobility and are hence expected to follow an Arrhenius law. Fig. 7(a) shows an Arrhenius representation of measured

\footnotetext{
${ }^{9}$ This is due to the particularly strong dimer bond energy for that system (see Section 3.6).
} 
a) $\mathrm{T}=80 \mathrm{~K}$

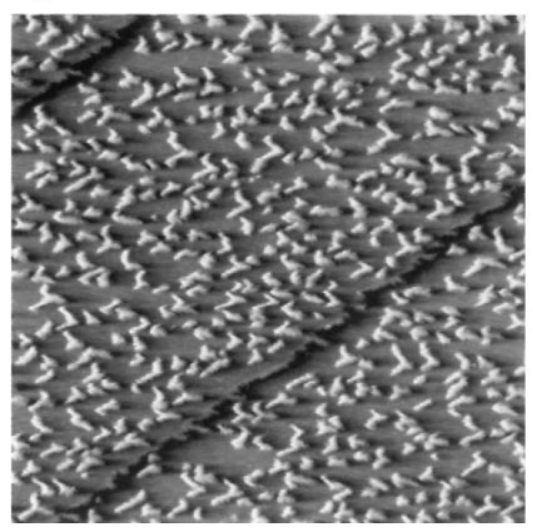

b) $\mathrm{T}=95 \mathrm{~K}$

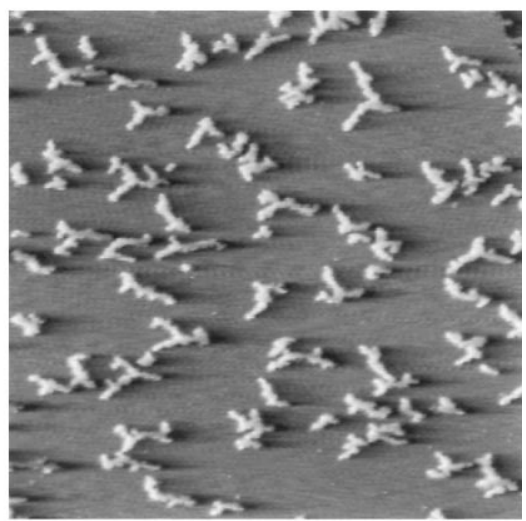

c) $\mathrm{T}=110 \mathrm{~K}$

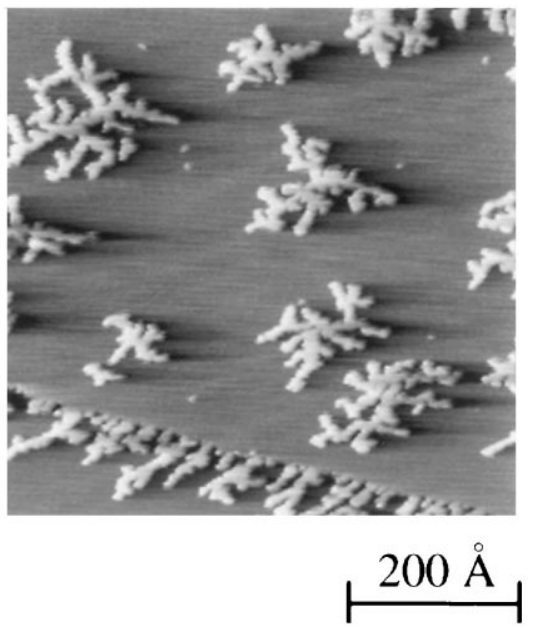

Fig. 6. Variation of the saturation island density with temperature for deposition of $0.12 \mathrm{ML}$ Ag onto a Pt(1 11$)$ surface at 80 , 95 , and $110 \mathrm{~K}$, respectively $\left(F=1.1 \times 10^{-3} \mathrm{ML} / \mathrm{s}\right)$.

saturation island densities for $\mathrm{Ag} / \mathrm{Pt}\left(\begin{array}{lll}1 & 1 & 1\end{array}\right)$ at a coverage of $0.12 \mathrm{ML}$ in comparison with self-consistent mean-field calculations. From the experimental data it is seen that there is a linear regime for temperatures from $110 \mathrm{~K}$ down to about $75 \mathrm{~K}$. At lower temperatures a downward bending of the island densities is measured. The linear regime reflects the expected power law expressed in Eq. (8). The downward bending to lower island densities is due to the fact that for lower temperatures, diffusion becomes slow with respect to deposition (at $D / F<10^{5}$ ) leading to a certain density of monomers left after deposition. Since in most experiments the surface is examined at the deposition temperature, and also some time after deposition, the monomers continue to be mobile after atoms have stopped arriving at the surface. They then either nucleate new islands or attach to existing ones without the presence of an incoming adatom flux. These effects were called post-nucleation and post-growth, and will be addressed in more detail below. 

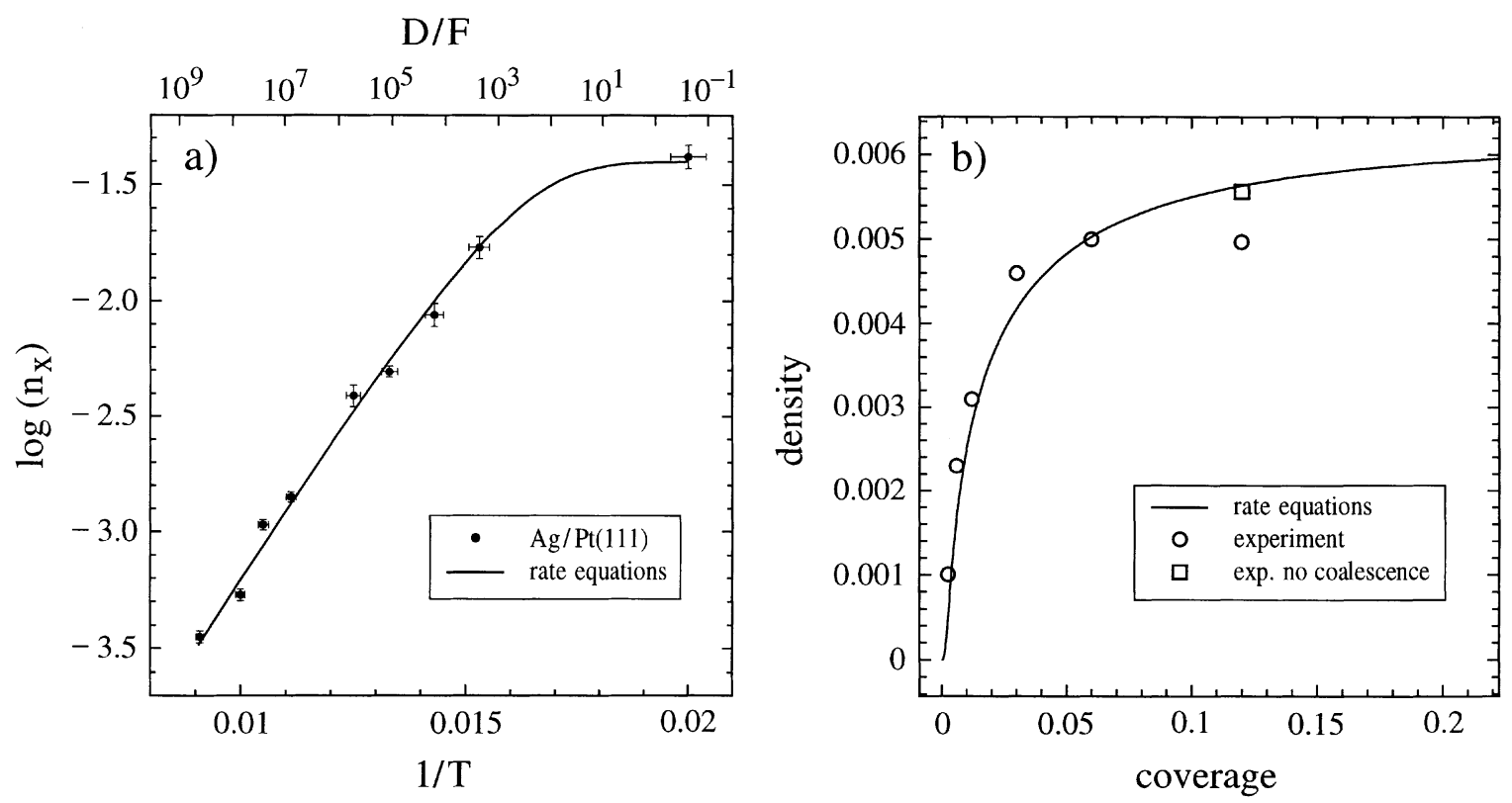

Fig. 7. Comparison of experimental island densities for $\operatorname{Ag} / \operatorname{Pt}\left(\begin{array}{lll}1 & 1 & 1\end{array}\right)$ with self-consistent calculations from mean-field nucleation theory. (a) Arrhenius plot of saturation island densities $(\Theta=0.12 \mathrm{ML})$ for the regime where dimers are stable nuclei. (b) Island density vs. coverage at $75 \mathrm{~K}$ (from Ref. [85]).

The solid line in Fig. 7(a) shows the best fit to the experimental data by mean-field rate equations which have been solved self-consistently [85]. Taking into account post-nucleation, the rate equations have been integrated until all monomers were gone, which only plays a role for $D / F<10^{5}$, or at $T<75 \mathrm{~K}$ for the present system. The mean-field calculation shows excellent agreement with experiment; it has been performed for ramified islands with a migration barrier of $E_{\mathrm{m}}=168 \mathrm{meV}$ and an attempt frequency of $\nu_{0}=6.76 \times 10^{13} \mathrm{~s}^{-1}$. As integration of mean-field rate equations allows the inclusion of post-deposition effects, it generally enables comparison to experimental data over a wide range of $D / F$, yielding considerable precision for $E_{\mathrm{m}}$. In the present example, data over almost 10 orders of magnitude for $D / F$ can be analyzed, resulting in an uncertainty for $E_{\mathrm{m}}$ of only $\pm 5 \mathrm{meV}$ $( \pm 3 \%)$. Comparison with KMC simulations, similarly including post-deposition mobility, yields identical results (see Fig. 14). Both methods can be used to extract parameters for surface diffusion from the type of data shown in Fig. 7(a) with high accuracy. As a result, diffusion barriers can be measured very precisely from nucleation island densities. For a comparable precision, FIM experiments require a considerable number of observations of single adatom displacements.

We will now investigate how the easiest and most widely used approach, namely to apply the scaling law expressed in Eq. (8), compares with the more elaborate but mathematically or computationally much more demanding analysis described above. Usually, one analyzes the linear part in the Arrhenius plot and extracts the migration barrier and the attempt frequency from the slope of a linear regression to the data and its intersection with the ordinate. For our example, this far more simple approach yields fairly good agreement with the values obtained from the full analysis. Taking the experimental data for $1 \times 10^{5} \leq D / F \leq 4 \times 10^{8}$, one obtaines $E_{\mathrm{m}}=169 \pm 13 \mathrm{meV}$ and $\nu_{0}=3 \times 10^{13.0 \pm 0.75} \mathrm{~s}^{-1}$ (see line fit 
and shaded error region in Fig. 9). The larger error margin is a consequence of the smaller temperature regime that can be analyzed linearly. Great care must be taken when selecting the data attributed to the linear regime [85]. As can be seen from closer inspection of Fig. 7(a), the calculated curve is nowhere strictly linear. For $D / F>10^{5}$, however, the deviation from a straight line is much smaller than the statistical errors inherent in the data. The deviation from linearity becomes detectable only upon inspection of Fig. 7(a) under glancing angle. For $D / F<10^{5}$, on the other hand, the curve starts to bend considerably. As a consequence, barrier values which are generally too small are obtained when linearly analyzing data in that regime. An example is the slightly lower barrier of $E_{\mathrm{m}}=157 \pm 7 \mathrm{meV}$ $\left(\nu_{0}=0.8 \times 10^{13.0 \pm 0.45} \mathrm{~s}^{-1}\right)$ that was obtained for the case of $\mathrm{Ag} / \mathrm{Pt}\left(\begin{array}{lll}1 & 1 & 1\end{array}\right)$ when island densities were analyzed down to $65 \mathrm{~K}\left(D / F=2 \times 10^{3}\right)$ [59]. Taking into account the low $D / F$ data falsifies the analysis as post-nucleation and other deviations from scaling already significantly decrease island densities (see Fig. 7(a)). We therefore emphasize that only data for $D / F>1 \times 10^{5}$ should be analyzed by a linear regression.

The evolution of the island density with coverage for our example of $\mathrm{Ag} / \mathrm{Pt}\left(\begin{array}{lll}1 & 1 & 1\end{array}\right)$ at $75 \mathrm{~K}$ is plotted in Fig. 7(b). The initial slope yields information on the mean island size during the pure nucleation phase from which the stable cluster size can be inferred [59]. The self-consistent rate equation analysis again shows perfect agreement with experiment. Since the analysis does not account for coalescence, an experimental value at $0.12 \mathrm{ML}$ for the (hypothetical) island density without coalescence was derived. This can be accomplished, since coalesced islands are differentiated from those that grew from a single nucleus by their shape.

In summary, the straightforward analysis of island densities by mean-field nucleation theory using Eq. (8), when performed for $D / F>10^{5}$ and a critical nucleus size of one, allows the determination of the energy barriers and attempt frequencies for surface diffusion with reasonable accuracy. This precision can be increased considerably when comparing experimental data either to self-consistent mean-field theory or to KMC simulations, which both are fully consistent to each other. In these calculations, post-deposition mobility can be included enabling comparison to experimental island densities over a larger regime of $D / F$.

\subsection{Critical cluster size, dimer mobility, and Ostwald ripening}

\subsubsection{The critical cluster}

The concept of a critical cluster, in the present context of kinetic limitations, is a dynamic one referring to the timescale of deposition. A critical cluster turns into a stable one upon incorporation of one extra atom. Then growth of the cluster becomes more likely than decay during the course of deposition. There are various ways to determine the critical cluster size for a given range of temperatures and deposition fluxes. For this purpose often the flux dependency of the saturation island density is consulted. Experiments were shown to discern that way critical cluster sizes on isotropic substrates [77]. The flux dependency equally enables determination of the dimension of diffusion on anisotropic surfaces [102], since the scaling exponent in Eq. (8) changes from $\chi=\frac{1}{3}$ to $\frac{1}{4}$ in going from $2 \mathrm{D}$ to $1 \mathrm{D}$ diffusion at $i=1$ (see Section 4.1 ). The critical cluster size can equally be inferred from island size distributions. Upon scaling, these distributions all fall onto common curves that only depend on $i[67,70,73,74,103]$. The first STM experiments that showed this scaling were reported by Stroscio and Pierce [75]. Soon after, Chambliss and Johnson [79] demonstrated scaling of island sizes in the presence of exchange nucleation where an incoming adatom is immobilized by the exchange and thus 
constitutes by itself a stable nucleus $(i=0)$. Identical scaling was reported for islands generated in the post-deposition phase where monomers are again stable on the timescale of deposition [77]. A further way to extract $i$ has already been addressed above. It consists in the inspection of the mean island size in the very early stages of nucleation, where islands were shown to contain about three atoms if $i=1$ [59]. At higher temperatures, where island densities in the pure nucleation regime become extremely small, however, this access to the critical cluster size becomes less practical. A change in critical nucleus size also manifests itself in a change in slope of lines fitted to the saturation island densities when represented in an Arrhenius plot. ${ }^{10}$

A common feature of all these approaches to determine the critical cluster size is that they explicitly presuppose the validity of either nucleation or scaling theories. For experimental tests of these theories one needs an independent way to determine the $i=1$ regime. One such way is the measurement of the threshold temperature for the onset of Ostwald ripening, starting from an island population predominantly consisting of dimers [22,85]. This experiment is particularly valuable since it excludes both dimer dissociation and diffusion up to a distinct temperature.

As a starting point, one prepares a large density of small islands (mostly dimers). This can be achieved by deposition of $\sim 0.1 \mathrm{ML}$ at a temperature chosen such that there is little mobility within the time of deposition $\left(D / F<10^{3}\right)$. Under these conditions, monomers diffuse towards each other only after deposition, leading to a high density of small islands. Fig. 8(a) shows an example of this. The mean island size is $2.8 \pm 0.4$ atoms and islands are mostly dimers and trimers with a few larger ones. The same result is obtained when depositing at temperatures where diffusion is frozen and subsequently gently annealing the surface to activate diffusion. The mean island size expected from integrating the rate equations (5) and (6) (Section 2) for both cases is about three atoms [77], in agreement with experiments on square [77] and hexagonal lattices (see Ref. [59] and Figs. 8(a) and (f)).

After preparation of the population of small islands, their density is monitored by STM as a function of annealing temperature. As the coverage is well known, one obtains information on the mean island size that way. One generally observes that the island size stays constant until, at a well-defined temperature, it suddenly increases. The constant regime implies that two things must not happen. The most fragile objects in the population, namely the dimers (and on square lattices also the trimers), do not dissociate, nor do they diffuse, since both would lead to coarsening.

In Fig. 8(f) there is a clear transition from a regime of constant island size to an exponential growth with annealing temperature. From the exponential increase it is likely that for the system $\mathrm{Ag} / \mathrm{Pt}\left(\begin{array}{lll}1 & 1 & 1\end{array}\right)$ coarsening is due 2D Ostwald ripening [105-107]. Ostwald ripening is caused by a more rapid dissociation of smaller islands in favor of larger ones. Through the availability of surface microscopy this phenomenon has received considerable attention in two dimensions [22,108,109]. The exponential increase in size is expected to continue as long as the rate limiting step is dissociation of atoms from one-fold coordinated sites at the island edge. For $\mathrm{Ag} / \mathrm{Pt}\left(\begin{array}{lll}1 & 1\end{array}\right)$ this is the case up to almost room temperature.

In our example, the onset of Ostwald ripening is located at $100 \mathrm{~K}$, therefore marking the threshold temperature for dimer dissociation for that system for annealing periods of half an hour. It yields an estimate of $320 \pm 20 \mathrm{meV}$ for the activation energy associated with the dissociation of a $\mathrm{Ag}$ dimer on

\footnotetext{
${ }^{10}$ The abruptness of this change in slope depends on the barriers for cluster dissociation compared to those for monomer migration [104].
} 
a) $50 \mathrm{~K}$
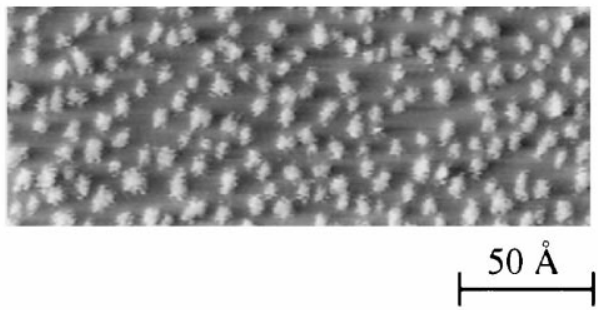

b) $110 \mathrm{~K}$
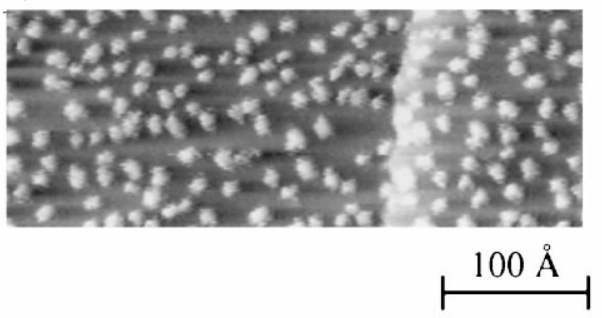

d) $230 \mathrm{~K}$
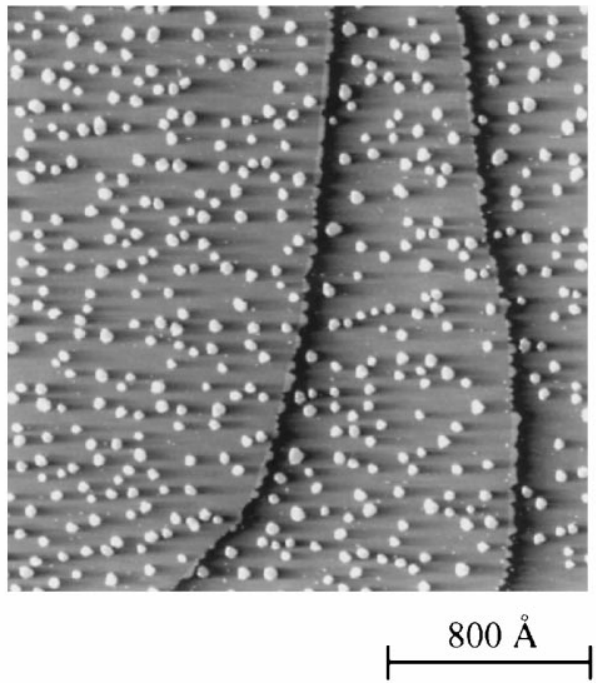

c) $140 \mathrm{~K}$

$\mathrm{n}=14$
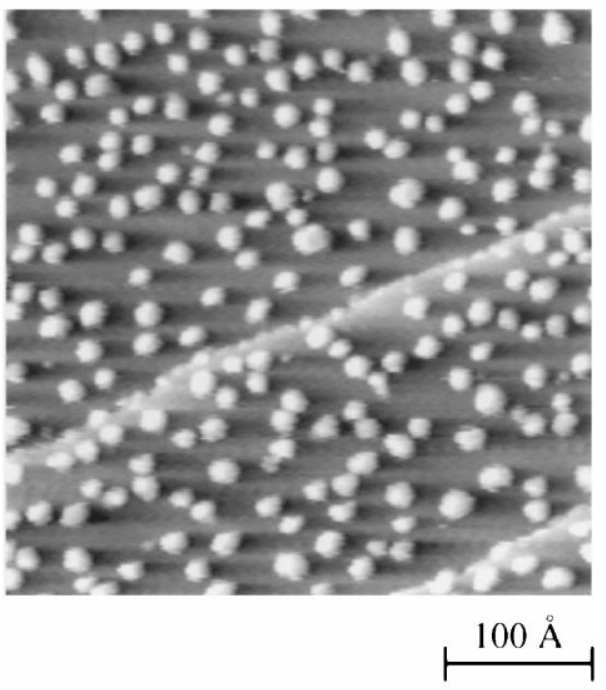

e) $280 \mathrm{~K}$
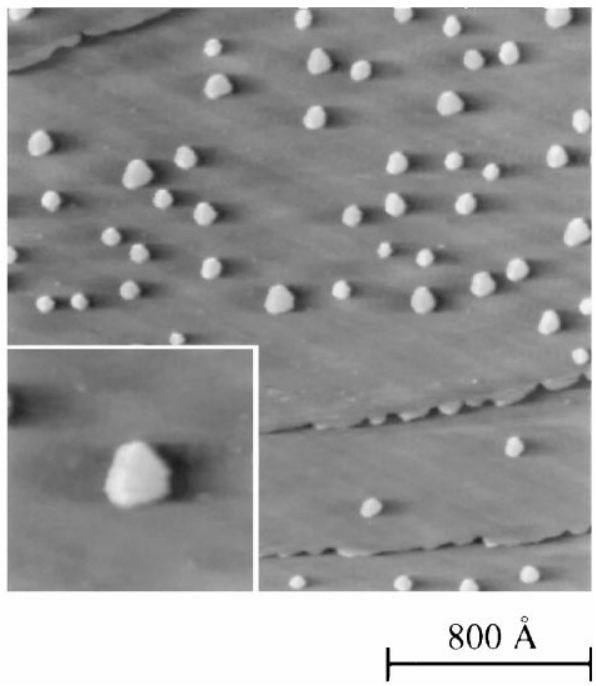

Fig. 8. STM images showing Ostwald ripening in two dimensions as a way to create compact 2D Ag islands with their size being well defined by the annealing temperature. The starting population of mostly dimers and trimers was produced via deposition of $0.1 \mathrm{ML} \mathrm{Ag}$ onto $\mathrm{Pt}\left(\begin{array}{ll}1 & 1\end{array}\right)$ at $50 \mathrm{~K}$. The onset of Ostwald ripening is marked by a sharp transition of constant to exponentially increasing island sizes as a function of annealing temperature. From the temperature and the annealing period the dimer bond energy and the temperature regime for $i=1$ can both be inferred (from Refs. [22,59]).

$\operatorname{Pt}\left(\begin{array}{lll}1 & 1 & 1\end{array}\right)$ (assuming the same prefactor as for diffusion). With $E_{\mathrm{m}}=168 \mathrm{meV}$ (see Section 3.1), the dimer bond energy therefore evaluates to $E_{\mathrm{b}}=150 \pm 20 \mathrm{meV}$. This value implies that on the timescale of deposition (typically $100 \mathrm{~s}$ for $0.1 \mathrm{ML}$ ), the $\mathrm{Ag}$ dimer is stable against dissociation and also immobile up to $110 \mathrm{~K}$; dimer instability should become detectable at $\sim 120 \mathrm{~K}$, where the lifetime of a dimer is about one second. 
f)

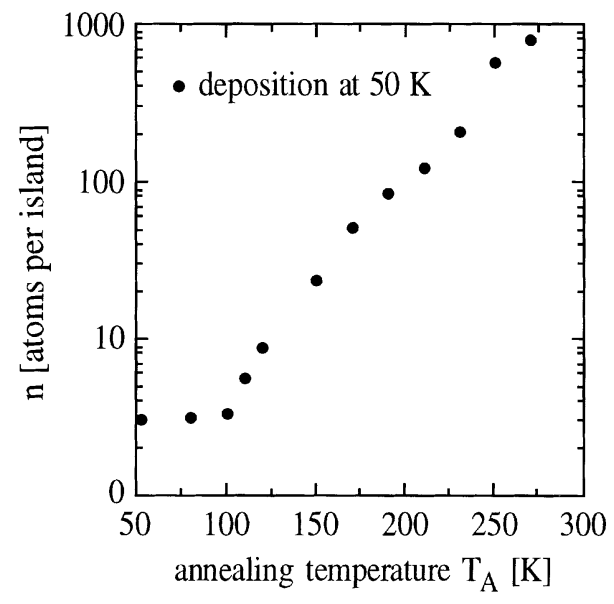

g)

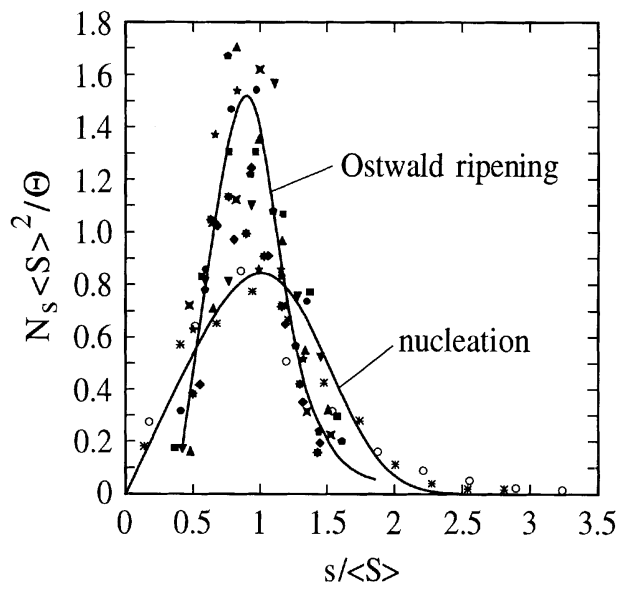

Fig. 8 (continued).

\subsubsection{Dimer mobility}

For nucleation studies it is important to know how dimer, or more generally, cluster migration affects island densities. This subject has been treated in Refs. [10,57]. As mentioned above, the onset temperature of island coarsening serves to exclude both dimer instability and diffusion. When working outside the regime of irreversible growth $(i=1)$ and immobile clusters, the question arises whether nucleation densities for a given system will first become perturbed by dimer diffusion or by dimer dissociation. This question has shortly been a matter of debate [110], but it seems to be clarified by a recent study [81]. Liu et al. [110] argued that dimer mobility should routinely be considered in the analysis of experimental data. The authors verified the scaling law from mean-field nucleation theory for fast dimer diffusion through simulations. They then suggested that several experimental results for metal-on-metal epitaxy ought to be re-interpreted in the light of dimer diffusion. However, soon after this study Bartelt et al. [81] showed that the scaling of island densities becomes only affected if the dimer diffusion rate is larger than its dissociation rate divided by the density of stable islands $n_{x}$. Since $n_{x}$ is typically of the order $10^{-4}$ to $10^{-3}$, this condition requires a much smaller barrier for dimer diffusion than for dimer dissociation in order for dimer diffusion to perturb nucleation. As a matter of fact, none of the systems considered by Liu et al. as candidates for dimer diffusion shows clear evidence for modified scaling due to dimer mobility [81]. They are much more reasonably interpreted as scaling at $i \geq 1$. In KMC simulations it was found that the barrier for dimer diffusion must come rather close to that for monomer diffusion in order to affect nucleation densities [37]. A good example showing that $i=1$ scaling can persist in the presence of dimer diffusion is $\mathrm{Si} / \operatorname{Si}(100)$. For that system dimer diffusion can be observed on the timescale of seconds from 300 to $400 \mathrm{~K}$ [35], nevertheless $i=1$ density scaling holds up to $555 \mathrm{~K}$ [76].

Therefore for most systems dimer diffusion is not expected to change island density scaling before dimer dissociation does. Nevertheless dimer diffusion is an interesting phenomenon in its own right and we will briefly address this issue based on knowledge from FIM [34,111,112] and on some theoretical results. We will also show systems where dimer diffusion possibly influences island densities. 
Whether one expects dimer diffusion before dissociation strongly depends on lattice geometry. On hexagonal lattices, calculations with effective medium theory (EMT) [113,114] suggest that dimers rapidly rotate around the substrate atom located closest to the dimer bond. ${ }^{11}$ Throughout this movement the dimer atoms stay confined to the six hollow sites adjacent to this substrate atom, and so there is no net translation associated with this rotation. Such an intracell movement was experimentally observed by FIM for Ir-dimers on $\operatorname{Ir}\left(\begin{array}{lll}1 & 1 & 1\end{array}\right)$ [117]. In contrast to this "easy" rotation, a center of mass translation of the dimer is associated with much higher barriers than monomer diffusion. Nevertheless, the fact that cluster diffusion is observable for $\operatorname{Ir}_{n} / \operatorname{Ir}\left(\begin{array}{lll}1 & 1 & 1\end{array}\right)$ [117] clearly indicates that dissociation sets in well after diffusion. The experiments for $\mathrm{Ag} / \mathrm{Pt}\left(\begin{array}{lll}1 & 1 & 1\end{array}\right)$ discussed in Fig. 8 suggest for this system that dimers dissociate before they could start to diffuse. Although no general rule can be derived on whether dissociation or diffusion of dimers appears first on hexagonal lattices, for the systems studied so far dimer dissociation clearly terminates the $i=1$ regime before dimer translation could start to influence the nucleation behavior.

The situation for dimer diffusion and dissociation is quite different on square lattices. Here dimer migration may indeed alter island densities before dimer dissociation does. This is expected for systems with exchange diffusion (such as Pt [118] and Ir [119]). There, dimer diffusion may have an even lower activation energy than monomer diffusion, as found for example by FIM for Pt(100) self-diffusion [111] and in ab initio calculations for $\mathrm{Al}\left(\begin{array}{lll}1 & 0 & 0\end{array}\right)$ [120]. On the other hand, for square surfaces with ordinary hopping diffusion, such as $\operatorname{Ag}\left(\begin{array}{lll}1 & 0 & 0\end{array}\right)$ [121], the barrier for dimer diffusion seems to be larger than for monomers [112]. This is in line with nucleation experiments showing a regular $i=1$ behavior without dimer diffusion for $\mathrm{Ag} / \mathrm{Ag}\left(\begin{array}{lll}1 & 0 & 0\end{array}\right)$ [87] and $\mathrm{Cu} / \mathrm{Ni}\left(\begin{array}{lll}1 & 0 & 0\end{array}\right)$ [77] which are both systems with ordinary hopping diffusion. In contrast to these experimental findings, a theoretical study proposed dimer shearing giving rise to considerable dimer mobility even for systems with hopping diffusion [122]. This mechanism, however, has so far not been confirmed experimentally.

For channeled planes as fcc(110) and bcc(2 11) (see $\operatorname{Re} / \mathrm{W}(211)$ [99]) considerable mobility is expected for "long" dimers oriented perpendicular to the channels, whereas in-channel dimers presumably stay immobile up to their dissociation.

In spite of chemical differences the general trends for dimer diffusion as a function of lattice symmetry are the following: dimer diffusion is expected to play a minor role on density scaling on hexagonal lattices, although there is presumably a rapid dimer rotation; on square lattices dimer (and trimer) diffusion may be fast enough to influence the nucleation behavior if there is exchange diffusion; on channeled surfaces dimer diffusion may also be of relevance for the analysis of nucleation data. The onset of dimer diffusion (and/or dissociation) in any of these cases is most easily detected by the annealing experiment described above. Island size distributions are sensitive indicators for dimer mobility, too. They become significantly sharper through cluster motion. This change to the size distributions appears already before dimer diffusion could alter the scaling of island densities [81].

\footnotetext{
${ }^{11}$ The energy barrier associated with this movement is of the order of, or even lower than that for monomer migration (EMT results: 86 vs. $80 \mathrm{meV}$ for Ag-dimer rotation vs. Ag-monomer migration on $\mathrm{Pt}(1111)$, and $63 \mathrm{vs}$. $66 \mathrm{meV} \mathrm{for} \mathrm{Ag/Ag(1} 11)$ ). A real translation of dimers, on the other hand, costs more activation energy (EMT: $137 \mathrm{meV} \mathrm{for} \mathrm{Ag} / \mathrm{Pt}$ and $113 \mathrm{meV}$ for $\mathrm{Ag} /$ $\mathrm{Ag}\left(\begin{array}{ll}1 & 1\end{array}\right)$ ). Note these results are in contrast to former EMT calculations performed for $\mathrm{Pt} / \mathrm{Pt}\left(\begin{array}{lll}1 & 1\end{array}\right)$, there rotation and translation have similar barriers $(160 \mathrm{meV})$ which are larger than $E_{\mathrm{m}}(130 \mathrm{meV})$, see Ref. [115]. Recent ab initio results for Al dimers on $\mathrm{Al}\left(\begin{array}{l}1 \\ 1\end{array} 1\right)$, however, fully confirm the picture suggested from the above EMT results: Al dimers rapidly rotate $(40 \mathrm{meV})$ whereas translation has a much higher activation barrier (120 meV) than monomer diffusion (40 meV) [116].
} 
In addition to the information on critical cluster sizes and dimer bond energies, 2D Ostwald ripening such as shown in Fig. 8 also serves to create compact islands of any arbitrary size in large abundance and with homogeneous spatial distribution [22]. This is of particular importance, since currently much experimental and theoretical effort focuses on exploring the evolution of chemical and physical properties of small agglomerations of matter as a function of the number of atoms they contain [123]. As an advantage to island nucleation, the size distributions obtained from Ostwald ripening are significantly sharper, as becomes evident from inspection of the STM images and the size distributions shown in Fig. 8. Similar to nucleation, the island size distributions obtained from Ostwald ripening at various temperatures (see the different symbols) become congruent when scaled with respect to the average size.

\subsection{Consistency tests of nucleation and scaling theories}

Recent STM experiments provide quantitative information on island densities and sizes under various deposition conditions. In this section, we will review those examples where a cross check between nucleation and scaling theory or where verification of these theories through comparison with experimental information that was independently obtained could be achieved.

The detemination of the stability range of the dimer $(i=1)$ in the Ostwald ripening experiment described in Section 3.2.1 does not rely on nucleation and scaling theories. Thus, it supplies additional information that serves as input to test predictions from these theories. One such prediction is the flux dependency expressed in Eq. (8). A flux series for the model system $\mathrm{Ag} / \mathrm{Pt}$ performed at $90 \mathrm{~K}$ yielded $\chi=0.32 \pm 0.05$ [85]. This confirms the exponent of $\frac{1}{3}$ predicted from nucleation theory, although the error margin is far too large to experimentally pin down the exponent of Eq. (8). This is a general problem in flux series caused by the rather weak influence of deposition flux on $n_{x}$, often being close to statistical errors. Therfore, precise quantification of scaling exponents are more conveniently arrived at using kinetic Monte-Carlo simulations, which allow for better statistics, as demonstrated for example in Fig. 4 of the preceding section.

From nucleation theory it is predicted when and how the slope of lines fitted to the saturation island densities in an Arrhenius plot due to a transition in critical cluster size should change. Both the temperatures where the change in slope appears for $\mathrm{Ag} / \mathrm{Pt}$, and the value for the slope in the $i=2$ regime, are consistent with the dimer bond energy extracted from the onset of Ostwald ripening (Fig. 9(a)) [85].

Fig. 9(b) shows the experimental island size distributions for $\mathrm{Ag} / \mathrm{Pt}$ in comparison with theoretical curves derived by Amar and Family [103] from scaling arguments for $i=1,2$, and 3 . The experimental island sizes obtained at 80 and $95 \mathrm{~K}$ fall into a common distribution. In addition, they agree well with the theoretical curve for $i=1$. Therefore, for $\mathrm{Ag} / \mathrm{Pt}$, the flux dependency, the change in slope due to dimer dissociation, and finally the island size distributions all fully confirm nucleation and scaling theory for the dimer bond energy independently obtained from coarsening experiments.

An experimental example establishing a cross link between nucleation and scaling theory was reported for $\mathrm{Cu} / \mathrm{Ni}\left(\begin{array}{ll}1 & 0\end{array}\right)$. The Arrhenius plot of saturation island densities shows a constant regime at low temperature attributed to post-nucleation (see Fig. 10). This is followed by an extended $i=1$ regime, from the slope of which the activation energy and attempt frequency for monomer migration could be determined to be $E_{\mathrm{m}}=0.36 \pm 0.03 \mathrm{eV}$ and $\nu_{0}=5 \times 10^{11 \pm 1} \mathrm{~s}^{-1}$. A second linear regime of $\log \left(n_{x}\right)$ vs. $(1 / T)$ was identified for tetramers being stable clusters. For square surfaces it is generally 

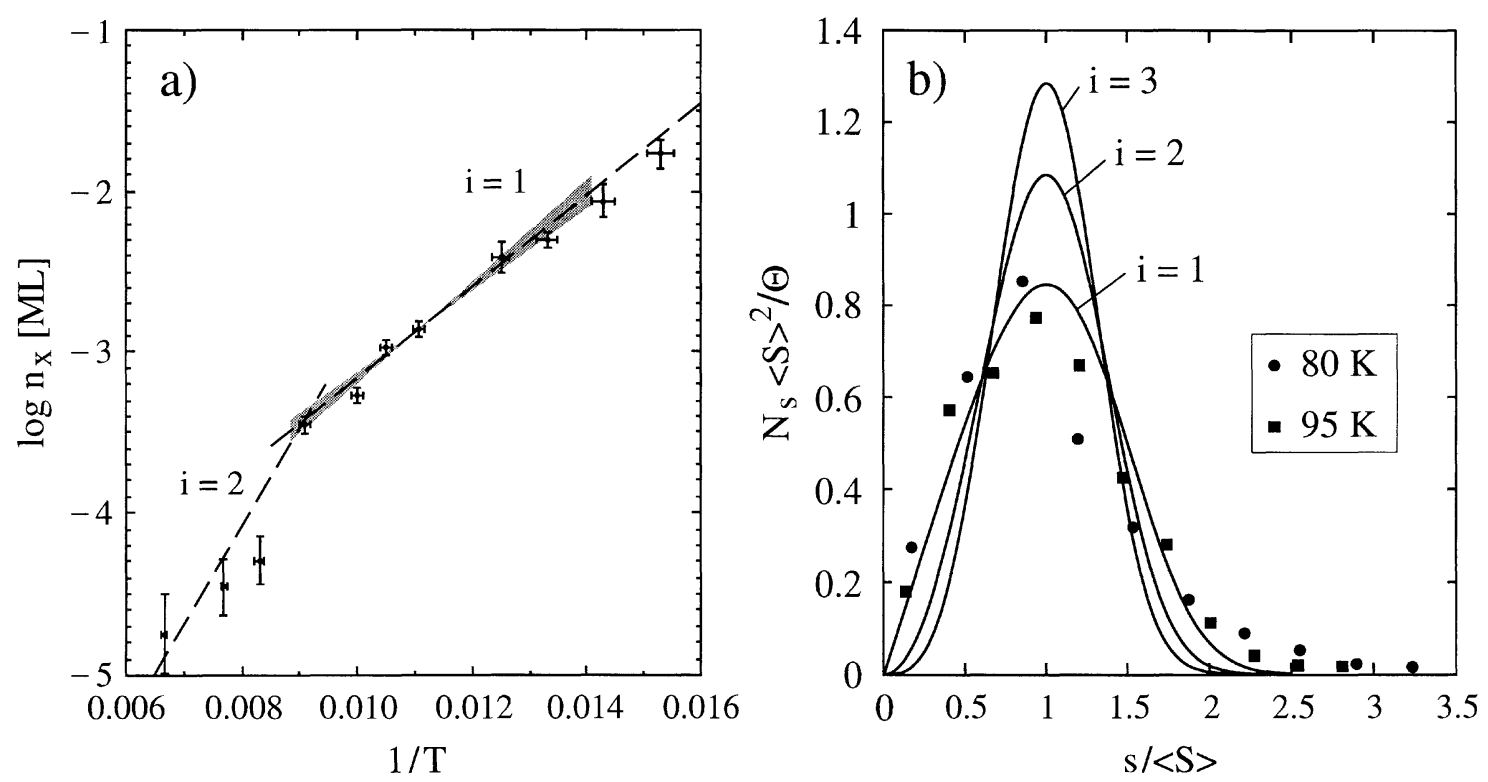

Fig. 9. (a) Arrhenius plot of saturation island densities $(\Theta=0.12 \mathrm{ML})$ for $\operatorname{Ag} / \operatorname{Pt}(111)$. The linear fit for $i=1$ $\left(75 \mathrm{~K} \leq T \leq 110 \mathrm{~K}\right.$ or $1 \times 10^{5} \leq D / F \leq 4 \times 10^{8}$ ) yields $E_{\mathrm{m}}=169 \pm 13 \mathrm{meV}$ consistent with the full analysis. The straight line for $i=2$ shows the slope from inserting the dimer bond energy of $E_{\mathrm{b}}=150 \pm 20 \mathrm{meV}$, derived from Fig. $8(\mathrm{~g})$, into Eq. (8). (b) Scaled island size distributions for $\mathrm{Ag} / \mathrm{Pt}$ in comparison with theoretical curves from Amar and Family (from Ref. [85]).

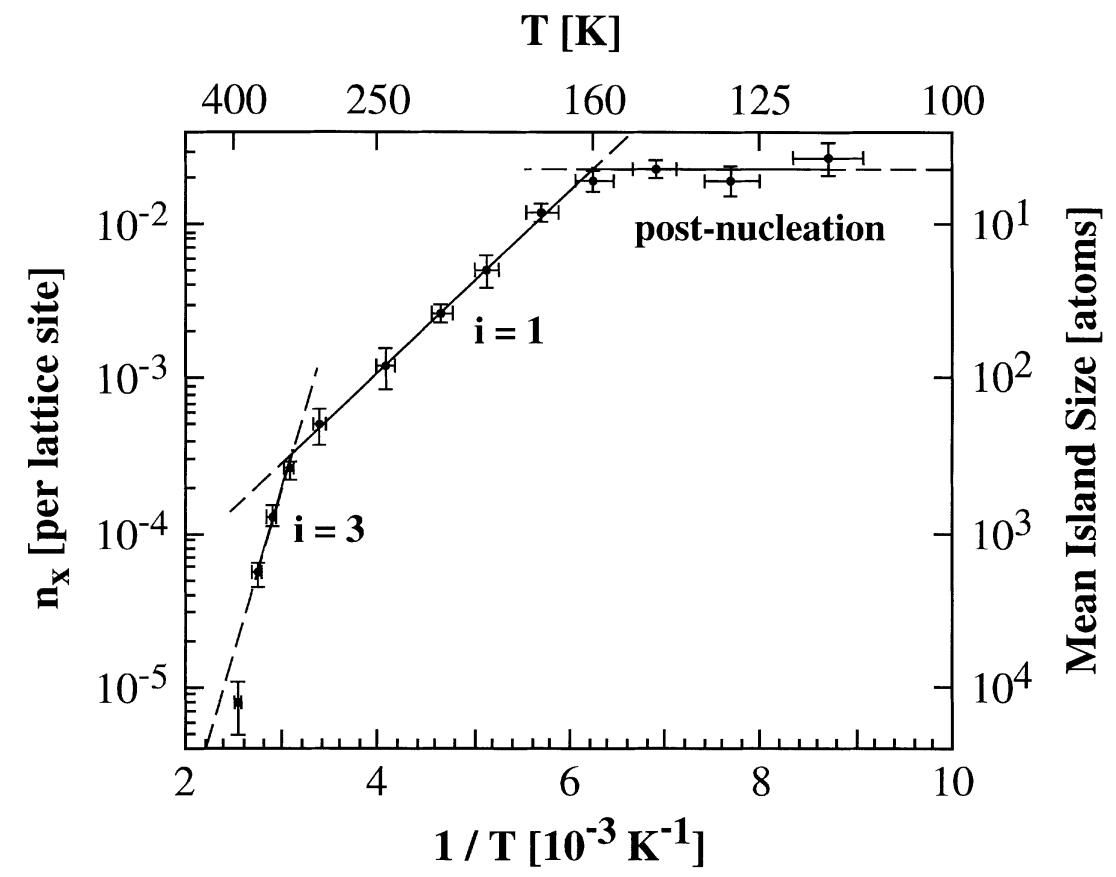

Fig. 10. Arrhenius plot of saturation island densities measured with variable temperature STM for Cu deposition onto $\operatorname{Ni}(100)\left(F=1.34 \times 10^{-3} \mathrm{ML} / \mathrm{s}, \Theta=0.1 \mathrm{ML}\right)$ (from Ref. [77]). 
a)

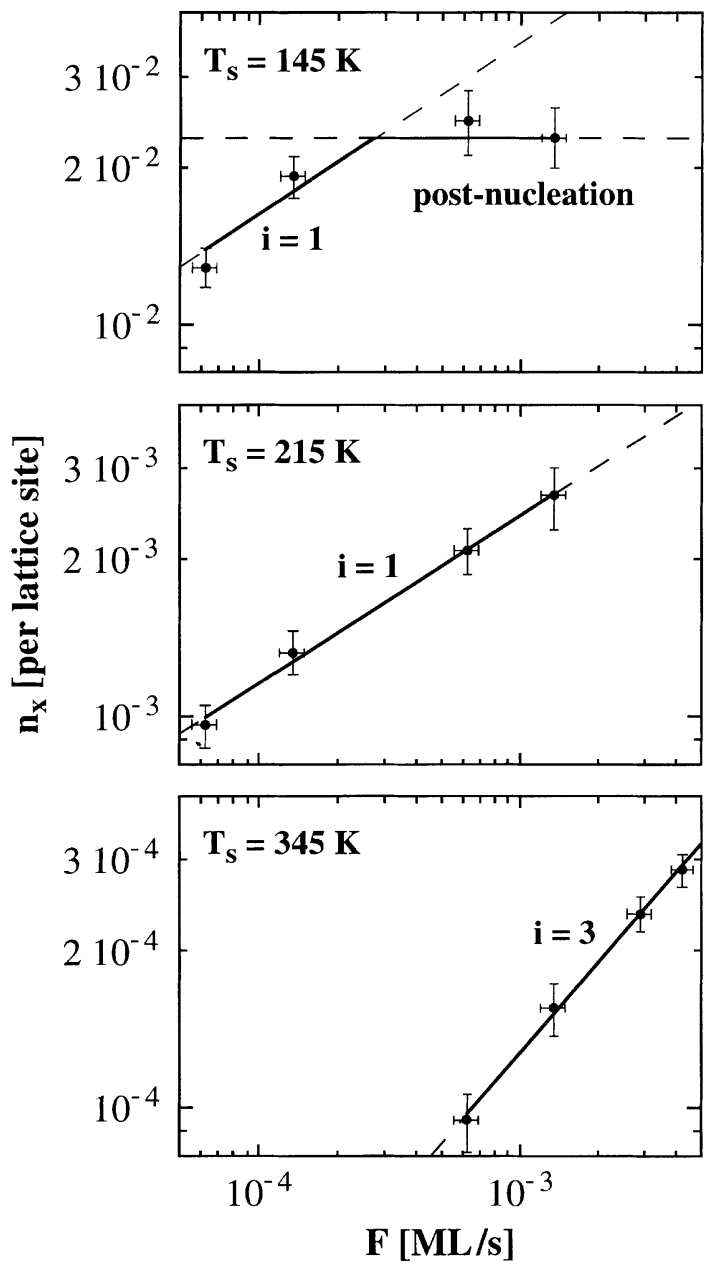

b)
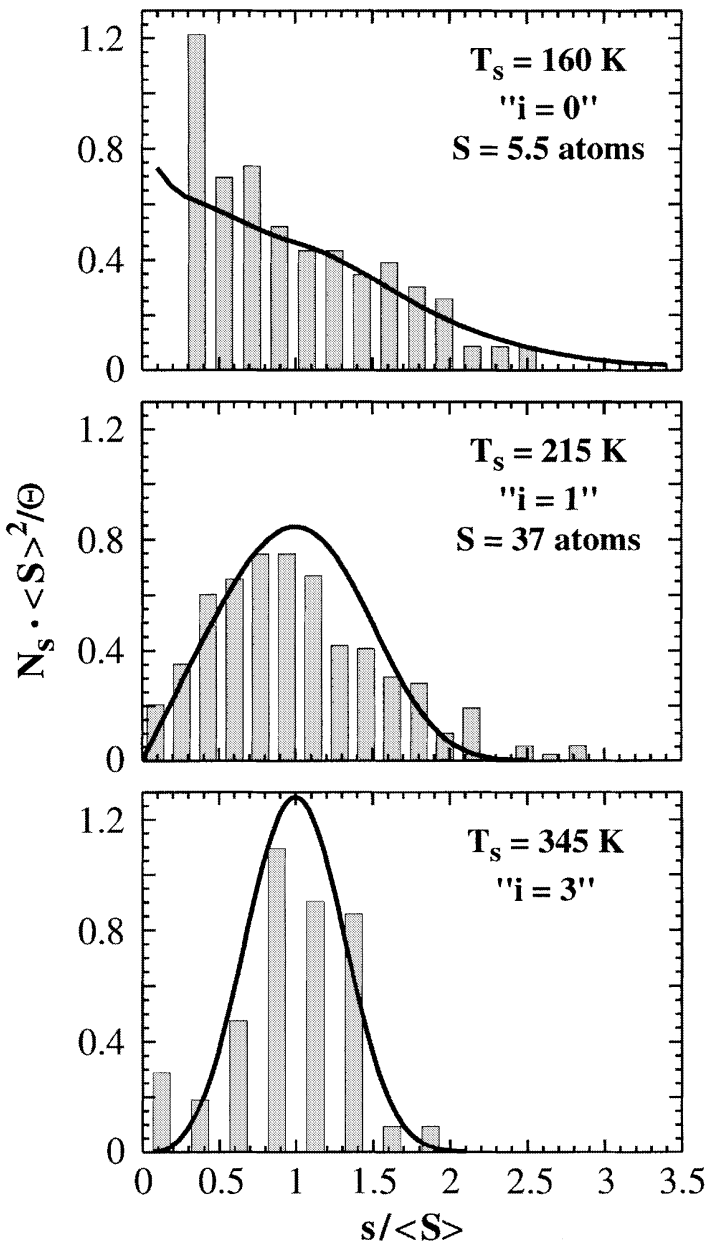

Fig. 11. Consistency of nucleation and scaling theory demonstrated for $\mathrm{Cu} / \mathrm{Ni}(100)$. (a) Double logarithmic plots of island densities $n_{x}$ vs. deposition flux $F$. The temperatures were chosen to lie right in the middle of the various nucleation regimes discerned in the Arrhenius plot in Fig. $10(\Theta=0.1 \mathrm{ML})$. (b) Scaled island size distributions for the respective temperatures in comparison with theoretical curves from Amar and Family (from Refs. [77,125]).

expected that trimers have a similar dissociation barrier to dimers, since both contain laterally only onefold coordinated atoms. Therefore, a direct transition from $i=1$ to $i=3$ was predicted [68] and experimentally verified by the data presented in Fig. 10. The sharpness of the transition in this system is caused by the large dimer bond energy of $E_{\mathrm{b}}=0.34 \pm 0.03 \mathrm{eV}$ compared to that for monomer migration [124].

The critical nucleus sizes indicated in Fig. 10 were inferred from measurement of the flux dependency shown in Fig. 11(a). The flux series confirm the theoretical scaling exponents expected from Eq. (8) with remarkable precision. The series performed at $215 \mathrm{~K}(i=1)$ yields $\chi=0.32 \pm 0.01$ vs. the theoretical exponent of $i /(i+2)=\frac{1}{3}$. The flux series taken at $345 \mathrm{~K}(i=3)$ results in an 
a)

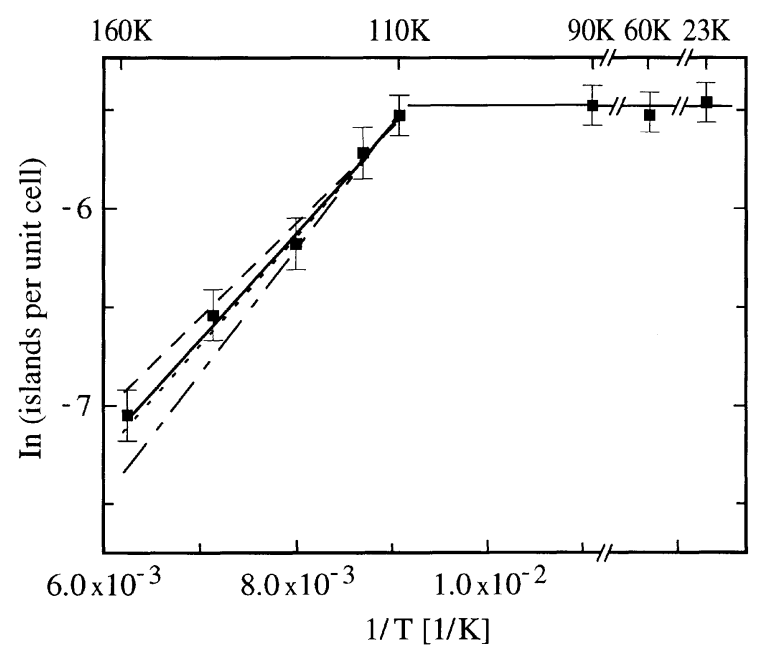

b)

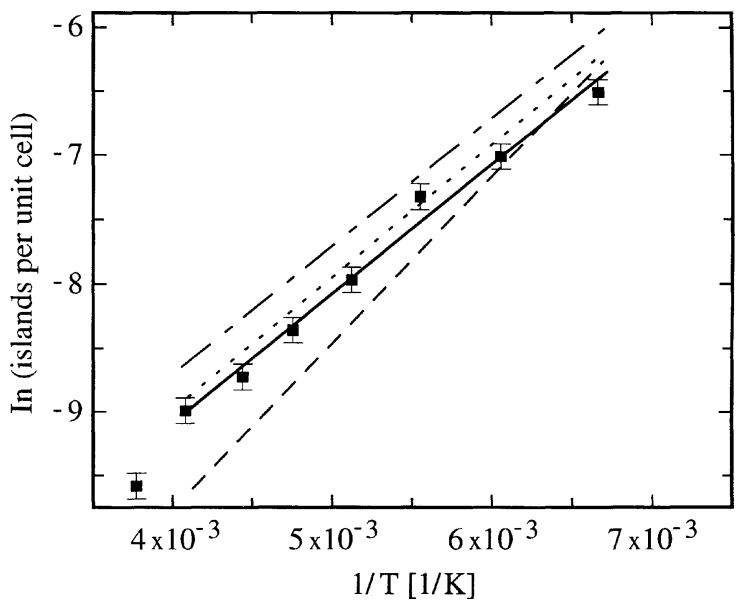

Fig. 12. Agreement of nucleation curve method with saturation island density method for $\mathrm{Pt} / \mathrm{Pt}\left(\begin{array}{lll}1 & 1 & 1\end{array}\right)$. (a) Island densities after deposition of $4.2 \times 10^{-3} \mathrm{ML}$ vs. 1/T (solid line: linear regression to experimental data, and dashed lines KMC for $E_{\mathrm{m}}=0.24, \quad 0.26$, and $0.28 \mathrm{eV}$, respectively). (b) Arrhenius plot of saturation island densities $(\Theta=0.1 \mathrm{ML}$, $F=6.6 \times 10^{-4} \mathrm{ML} / \mathrm{s}$ ). Linear regression (within $3 \times 10^{6} \leq D / F \leq 8 \times 10^{9}$ ) and application of Eq. (8) with $\chi$ for compact islands yields $E_{\mathrm{m}}=0.26 \mathrm{eV}$ (from Ref. [37]).

exponent of $\chi=0.58 \pm 0.02$ in comparison to $\frac{3}{5}$ expected from theory. This result in itself can be considered as a consistency test of nucleation theory. In addition, the island size distributions obtained at temperatures where $i$ was pinned down by flux series, agree with the predictions from scaling theory for the respective critical cluster sizes (see Fig. 11(b)). This result proves the consistency of scaling theories with mean-field nucleation theory.

A further experiment that enables a consistency test of nucleation theory has been performed for $\operatorname{Pt}\left(\begin{array}{lll}1 & 1 & 1\end{array}\right)$ homoepitaxy [37]. The authors of Ref. [37] have developed an alternative approach to evaluate $E_{\mathrm{m}}$ from island densities which they called nucleation curve method. Instead of measuring island densities at saturation coverages, they deposited much smaller amounts and measured the onset temperature of nucleation arising from the onset of diffusion. The slope of the island densities formed at these low converages was subsequently determined (see Fig. 12(a)). Since their analysis is independent of nucleation theory - they compare their results to KMC - it again serves as input to test nucleation theory. Their result is $E_{\mathrm{m}}=0.26 \pm 0.01 \mathrm{eV}\left(\nu_{0}=5 \times 10^{12 \pm 0.5} \mathrm{~s}^{-1}\right)$, again representing a value with remarkable precision $( \pm 4 \%)$; in addition it compares very well to the FIM result $\left(E_{\mathrm{m}}=0.25 \pm 0.02 \mathrm{eV}\right)[126]$.

The main differences between measuring a nucleation curve as opposed to measuring saturation island densities are the following. At the onset of monomer diffusion, dimers are normally stable and immobile, ${ }^{12}$ hence no additional information is required. Effects of island shapes and coalescence are excluded since the islands are small and therefore compact, no matter what the details of edge diffusion are, and they are far too small to coalesce.

\footnotetext{
${ }^{12}$ For exceptions to this rule see the discussion of dimer mobility in the preceding section.
} 
The authors of Ref. [37] also performed measurements of saturation island densities for $i=1$ thus enabling a test of their method against nucleation theory. A linear regression to these island densities and application of Eq. (8) yields exactly the same result within the error margin $\left(E_{\mathrm{m}}=0.26 \mathrm{eV}, \nu_{0}=3 \times\right.$ $\left.10^{12} \mathrm{~s}^{-1}\right)$ as the one obtained from the nucleation curve. This striking agreement is thus a further confirmation of the validity of nucleation theory and puts the analysis of nucleation island densities within standard nucleation theory at $i=1$ as a method to measure surface diffusion on a firm basis.

It should be noted that several arguments are raised by the authors of Ref. [37] as to why the analysis of saturation island densities with nucleation theory should be less reliable than their nucleation curve method, or at least require additional knowledge. For a detailed discussion of this issue we refer to a recent publication [85]. In brevity, the agreement between nucleation method and the "new method" demonstrated above is due to the fact that: (i) deviations from scaling are extremely small for the range of $D / F$ addressed here; (ii) coalescence effects become important only well after $0.1 \mathrm{ML}$; and (iii) island shapes have a minor effect on scaling exponents (these items become evident from Section 2). Nevertheless, we entirely agree with the authors that the weakness of certain approximations for $\sigma$, notably the geometric concept, yields inconsistent results, as well as that in the past there might have been some uncertainties on the range of applicability and precision of Eq. (8). These latter are sorted out by the results discussed in Section 2 and Ref. [85]. Therefore, the nucleation curve method of Bott et al. is an alternative way to extract quantitative information on diffusion from nucleation data; it is as equally valid as the classical nucleation method, where the saturation island density is measured at $i=1$ in a well-defined range of $D / F$ [59]. The consistency of both methods, together with the other experimental results described above, all underline the validity of the scaling law from classical nucleation theory. Its application for extracting diffusion parameters is thereby well established. ${ }^{13}$ Also scaling theories about island sizes became experimentally confirmed.

\subsection{Growth at $D / F \ll 10^{5}$ - from post-deposition mobility to statistical growth}

For deposition at very low temperatures diffusion can become very slow with respect to the incoming flux of adatoms. As a consequence, only a small fraction of the deposited adatoms have the time to create nuclei or attach to islands during the course of deposition and a considerable monomer density may be left after deposition has been terminated. Rapid cooling would preserve these remaining monomers and they would become detectable, for example in STM topographs, or in the scattered He or ion intensity. Usually, however, the surface is examined at deposition temperature, and - particularly in the case of STM - also some time after deposition. Therefore, monomers continue to diffuse leading to island growth or nucleation after the desired amount has been deposited. This evolution was labeled post-growth and post-nucleation (in the sense of island growth and nucleation taking place postdeposition). It was first noticed for $\mathrm{Ag} / \mathrm{Pt}\left(\begin{array}{lll}1 & 1 & 1\end{array}\right)$ [59] and examined in detail for $\mathrm{Cu} / \mathrm{Ni}\left(\begin{array}{lll}1 & 0 & 0\end{array}\right)$ [77]. The evolution of the surface morphology after interruption of deposition has earlier been addressed in context with the growth interruption technique in MBE [127]. By periodically interrupting the deposition flux, one leaves time for smoothening of the film by disintegration of small islands that might have formed on top of other islands. In variance to this 3D smoothening, post-growth and post-

\footnotetext{
${ }^{13}$ In favorable cases estimates on cluster binding energies may also be obtained. However, for $i>1$, island densities are generally small and one has to be aware of the presence of steps which impose finite size effects. One similarly has to worry about the reduced statistical significance of the data.
} 
a)

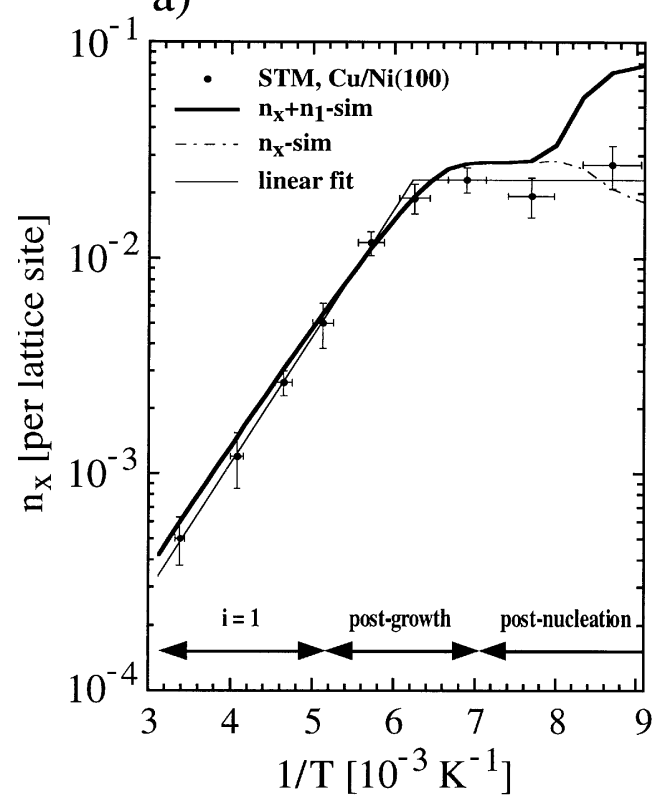

c)

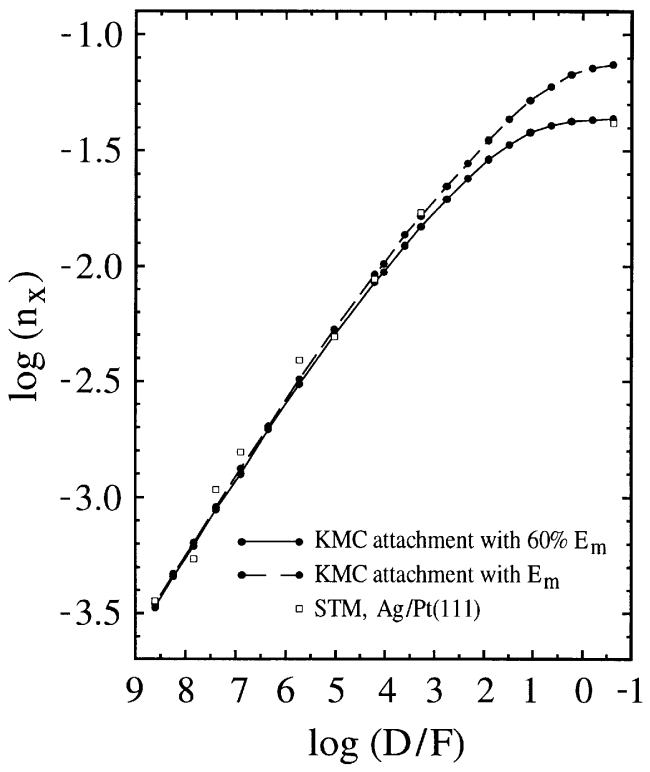

b)

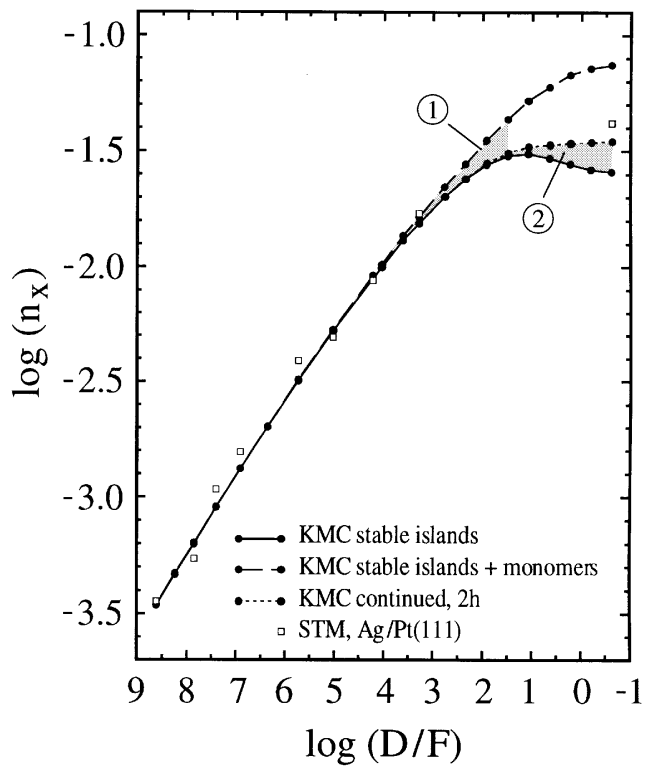

d)

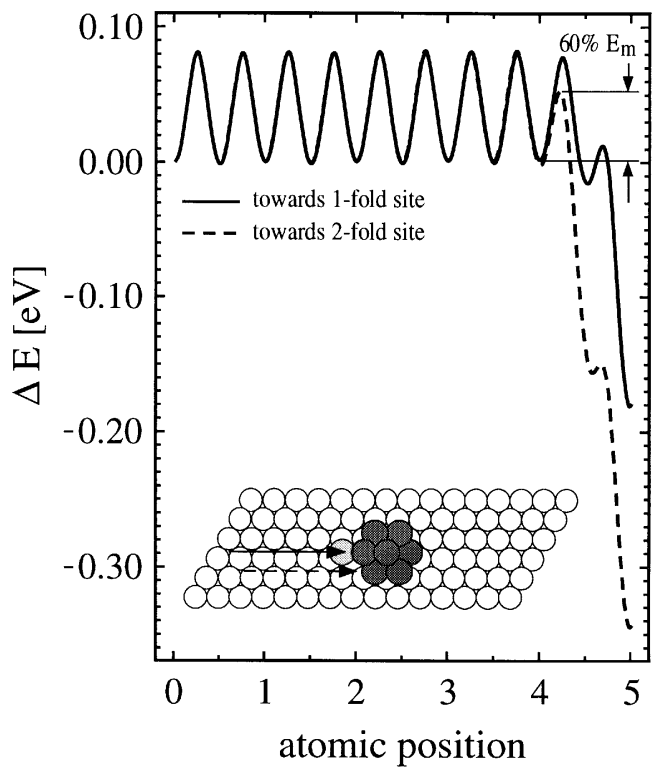

Fig. 13. Deviations from scaling and post-deposition mobility studied through comparison of calculated to experimental island densities. (a) The transition from dynamic nucleation (with $i=1$ ) to post-growth with decreasing temperature is well reproduced for the system $\mathrm{Cu} / \mathrm{Ni}\left(\begin{array}{lll}1 & 0 & 0\end{array}\right)$ by integrating rate equations (lattice approximation) until $2 \mathrm{~h}$ after deposition (from Ref. [77]). (b) Distinction between deviation from scaling and the effect of post-deposition mobility in comparison to experimental results for $\mathrm{Ag} / \mathrm{Pt}$. (c) Effect of easy attachment to islands equally explains the experiment for Ag/Pt. (b) and (c) KMC performed for dendritic islands with the diffusion parameters for $\mathrm{Ag} / \mathrm{Pt}(111)$. (d) EMT calculation for $\mathrm{Ag} / \mathrm{Pt}(111$ ) showing the potential energy for an atom approaching a one- and two-fold site at an island (from Ref. [85]). 
nucleation in the present context describe a purely 2D evolution of the surface morphology where remaining monomers become incorporated into islands. In the present section, we discuss deviations from scaling due to this $2 \mathrm{D}$ evolution, generally appearing for $D / F<10^{5}$. In the extreme case of $D / F<1$ one enters the regime of statistical growth where thermal mobility is frozen. This regime will also be addressed as it bears valuable information, for example, on transient mobility.

\subsubsection{Post-deposition mobility}

The transition from regular nucleation during deposition to post-growth and post-nucleation is displayed in Fig. 13(a) for the case of $\mathrm{Cu} / \mathrm{Ni}\left(\begin{array}{lll}1 & 0 & 0\end{array}\right)$ [77]. For $T>200 \mathrm{~K}$ (generally for $\left.D / F>10^{5}\right)$ the experimental island densities show the linear slope, correctly described by nucleation theory $(i=1)$. For lower temperatures, a downward bending to smaller island densities is observed (see theoretical curve), and finally the island densities become independent of deposition temperature, as evidenced by the plateau in Fig. 13(a). Both features generally characterize low temperature deposition. The downward bending is due to the fact that monomers remaining after interruption of the incoming deposition flux predominantly contribute to growth of existing nuclei, whereas under continued deposition they would have created further nuclei. Accordingly, this regime is referred to as postgrowth. The regime of constant island density, on the other hand, is explained by $D$ becoming sufficiently small with respect to $F$ such that only very few nuclei are formed during deposition with most nucleation taking place afterwards. This second regime was therefore called post-nucleation. The corresponding plateau in the Arrhenius plot can be rationalized as follows. In nucleation and growth, the deposition flux is the only parameter introducing time. Accordingly, all rates enter exclusively in relation to the flux. This is best seen by dividing the rate equations (5) and (6) in Section 2 by $F$ which yields $\mathrm{d} n / \mathrm{d} \Theta$ on the left-hand side and terms containing $D / F$ on the right-hand side. In the postnucleation regime $D / F$ is sufficiently small so that these terms become negligible with respect to the deposition term. Therefore the monomer density increases linearly and almost no stable islands form during deposition. The diffusion rate, respectively the substrate temperature, determine the time after deposition it takes to form all nuclei. The final density of nuclei, however, is a constant that only depends on coverage. A remarkable feature of island populations created by post-deposition mobility is their exponentially decreasing size distribution (see, for example, Fig. 11(b)). Deposition at very low temperatures therefore generally opens up the possibility of creating very small islands in high abundance.

For the quantitative analysis of experimental island densities and sizes obtained for small $D / F$ it is important to notice that remaining monomers are detectable, for example, by STM. ${ }^{14}$ Due to tip convolution they are hardly discernible from islands and thus would be counted as such. Accordingly, the results from the simulations in Fig. 13(a), similar to the case of $\operatorname{Ag} / \operatorname{Pt}\left(\begin{array}{lll}1 & 1 & 1\end{array}\right)$ (Fig. 7), show the number density of stable islands plus that of the remaining monomers. The solid line represents this sum obtained after integrating the rate equations until some time $(2 \mathrm{~h})$ after deposition. In analogy to the experiment the island densities at low temperatures lie below the scaling law and finally show a plateau where they become temperature independent.

Both deviations from scaling and post-deposition effects are present for small $D / F$. These two effects add up to yield the final island density seen in the experiment. They can be discerned using KMC results, such as shown in Fig. 13(b) where they are compared to experimental data for the

${ }^{14}$ Also other experimental techniques as SPA-LEED, HAS, or LEIS would reveal these monomers. 
$\mathrm{Ag} / \operatorname{Pt}\left(\begin{array}{lll}1 & 1 & 1\end{array}\right)$ system. ${ }^{15}$ There are three curves, displaying $n_{x}, n_{1}+n_{x}$, both directly after deposition, and $n_{1}+n_{x}, 2 \mathrm{~h}$ after deposition. The curves cannot be discerned from one another for $D / F>10^{5}$ indicating that post-deposition effects are absent or negligible in that range, i.e. diffusion is fast enough that nucleation and growth can entirely take place during deposition. Accordingly, the densities are well described by a straight line expected from Eq. (8).

Deviations from the linear scaling behavior appear first for $D / F<10^{5}$. From there on, the monomers left after deposition reach a detectable amount (see deviation of $n_{1}+n_{x}$ from $n_{x}$ indicated as hatched area 1). Note, however, $n_{x}$ directly after deposition stays congruent with the curve for KMC-continued until $D / F \approx 5 \times 10^{2}$. This signifies that for $10^{5}>D / F>5 \times 10^{2}$ the density of stable islands is determined immediately after deposition stops, deviations from scaling are intrinsic, and postdeposition mobility does not change island densities; it attaches monomers to existing islands without creating new ones (post-growth). For smaller $D / F, n_{x}$ reaches a maximum at $D / F \approx 5 \times 10^{1}$; afterwards it has a negative slope, i.e. smaller island densities at lower temperatures. This is due to diffusion becoming slower such that fewer and fewer islands can be created during deposition. Accordingly, post-deposition mobility is now responsible for the creation of new islands (postnucleation) lifting up the curve of $n_{x}$ to yield the characteristic plateau (see hatched area 2). The KMC results in Fig. 13(b) correctly describe the experiment for $\mathrm{Ag} / \mathrm{Pt}$ in the whole range of $D / F$. The simulations were performed with identical parameters as the analysis with rate theory in Fig. 7(a). The agreement of both methods is striking. The value of $D / F \approx 10^{2}$ for the transition from post-growth to post-nucleation can be inferred consistently from the intersections of linear fits to the experimental data for $\mathrm{Cu} / \mathrm{Ni}$ in the Arrhenius plots (Figs. 10 and 13(a)) and the flux dependency (Fig. 11(a)), as well as from the KMC simulations discussed above.

\subsubsection{Easy attachment}

From some of the low temperature data, there is evidence that monomers can be attached to islands through a mechanism other than ordinary terrace diffusion. Post-deposition mobility alone cannot explain the island densities reported for $\mathrm{Cu} / \mathrm{Ni}\left(\begin{array}{lll}1 & 0 & 0\end{array}\right)$ at $T \leq 130 \mathrm{~K}(D / F \leq 1)$ for example. These lie significantly below the rate equation result (see curve $n_{1}+n_{x}$ in Fig. 13(a)). These findings were interpreted as transient mobility [77]. Similar observations were made for $\mathrm{Ag} / \mathrm{Pt}$, where some of the low temperature data taken very soon after deposition had an island density too small to be accounted for by post-nucleation [85]. However, for $\mathrm{Ag} / \mathrm{Pt}$ transient mobility can be excluded (see below). Therefore, we discuss an alternative way to rationalize these experimental findings in a consistent manner. This consists of attachment of monomers to islands with a smaller activation energy than that for terrace diffusion. 16

There are several indications that such "easy" attachment indeed exists. Wang and Ehrlich [129] reported denuded zones bare of deposited Ir adatoms around Ir islands on $\operatorname{Ir}\left(\begin{array}{llll}1 & 1 & 1\end{array}\right)$. Although depletion zones around islands or steps are a general feature when diffusion determines the growth rate, in the experiments of $\operatorname{Ir} / \operatorname{Ir}\left(\begin{array}{lll}1 & 1 & 1\end{array}\right)$ these zones were found under conditions where ordinary terrace diffusion is

\footnotetext{
${ }^{15}$ The experimental data were normalized to $D / F$ such that the results discussed in Figs. 13(b) and (c) apply for the general case.

${ }^{16}$ An alternative explanation is the interaction of the STM tip with adsorbed monomers. For $\mathrm{Pt} / \mathrm{Pt}(111)$, the influence of the STM tip was shown to considerably decrease the diffusion barrier [37]. This explanation implies that some of the remaining monomers might have been attached to existing islands due to the measurement itself. For $\mathrm{Ag} / \mathrm{Pt}$ such effects can be excluded as monomers can be imaged repeatedly without inducing mobility at $35 \mathrm{~K}$ [128].
} 
frozen, i.e. at $50 \mathrm{~K}$. They even persisted in a second series of FIM experiments performed at $20 \mathrm{~K}$ [130]. The observation was interpreted as a reduced corrugation of the (attractive) adsorption potential close to an ascending step, permitting atoms to thermally diffuse towards an island even at temperatures as low as $20 \mathrm{~K}$. This "easy" attachment was also seen in He scattering experiments for $\mathrm{Ag} / \mathrm{Pd}\left(\begin{array}{lll}1 & 0 & 0\end{array}\right)$ which indicated "neighbor driven mobility" over one site activated down to a temperature half that of the onset temperature for regular diffusion [131]. In line with these experiments, several calculations report a smaller barrier for the last jump towards an island [132,133]. This attraction towards islands over one lattice distance can be understood in terms of strain fields around steps or adsorbate-adsorbate interactions presumably mediated by the substrate.

The KMC results reproduced in Fig. 13(c) demonstrate the effect on island densities when attachment towards islands and monomers is performed with a reduced barrier of $60 \%$ of the terrace value. This amount is suggested from EMT calculations for attachment towards two-fold coordinated sites for $\mathrm{Ag} / \mathrm{Pt}\left(\begin{array}{lll}1 & 1 & 1\end{array}\right)$ (see Fig. 13(d)) or $\mathrm{Pt} / \mathrm{Pt}(111)$ [134]. The island density detectable in the experiment $\left(n_{1}+n_{x}\right)$ becomes smaller due to the effect of "easy" attachment over one site. The most prominent effect is at low temperatures. For $D / F>10^{5}$, easy attachment has only negligible effect, which has the important implication that these details are unimportant for the analysis within the scaling regime. The results in Fig. 13(c) describe the experiment for $\mathrm{Ag} / \mathrm{Pt}\left(\begin{array}{lll}1 & 1 & 1\end{array}\right)$ quite well. It should be noted that easy attachment is overestimated in the KMC simulations as there was no distinction between attachment towards one- and two-fold coordinated edge sites. The barrier of the last diffusion step (always fcc $\rightarrow$ fcc) towards a one-fold site is found to be not as strongly reduced as towards a twofold site (Fig. 13(d)). Nevertheless the simulations demonstrate how the details of attachment to islands and neighbor driven mobility influence island densities. The available low temperature data for $\mathrm{Cu} / \mathrm{Ni}\left(\begin{array}{lll}1 & 0 & 0\end{array}\right)$ and $\mathrm{Ag} / \mathrm{Pt}\left(\begin{array}{lll}1 & 1 & 1\end{array}\right)$ can be explained by a combination of post-deposition mobility and attachment to islands with a reduced barrier.

\subsubsection{Statistical growth}

Deposition at temperatures where thermally activated diffusion is frozen leads to statistical growth. STM measurements in this regime (see Fig. 14(a)) allow the determination of the mean island sizes as quotient of coverage and density. Both numbers are known with sufficient absolute precision that conclusions on transient mobility and/or easy attachment to islands can be reached. This is achieved by experimentally following the mean island size with increasing coverage and making comparison to numbers expected from models for the respective cases. For pure statistical growth the mean island size expected for the various lattice types can be calculated with simple mathematics [135,136]. These percolation theories neglect deposition onto filled sites, however. Therefore, they do not account for impingement onto, and subsequent downward diffusion from, monomers and small islands. Consequently, they yield numbers that are slightly too small compared to epitaxial growth experiments. These effects can be accounted for, either by integrating the rate equations ((5) and (6) in Section 2) for no diffusion, or in Monte-Carlo simulations. Results from both methods obtained for a hexagonal lattice are displayed in Fig. 14(b), again showing that KMC is fully consistent with rate theory. The mean island size expected for deposition of $0.1 \mathrm{ML}$ under conditions where atoms stick to their impact site or roll down from another adatom is 1.48 atoms (percolation theory yields only 1.35 atoms).

If one permits transient mobility over one site, or easy attachment towards islands or monomers over one site, the expected mean island size is 2.3 atoms at $0.1 \mathrm{ML}$, which is considerably larger than the value without these effects. It is important to realize that attachment to islands and transient mobility 
a) $\mathrm{T}=35 \mathrm{~K}, \quad \Theta=0.1 \mathrm{ML}$

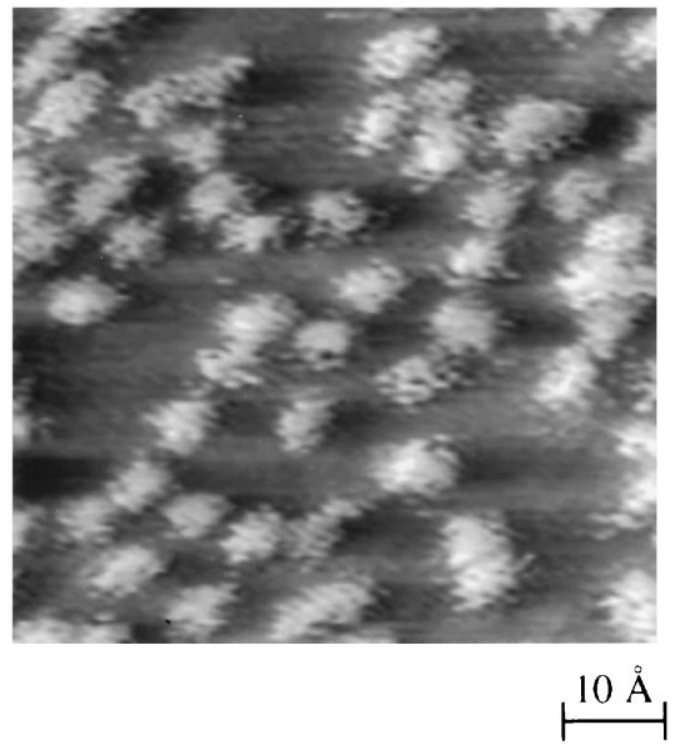

b)

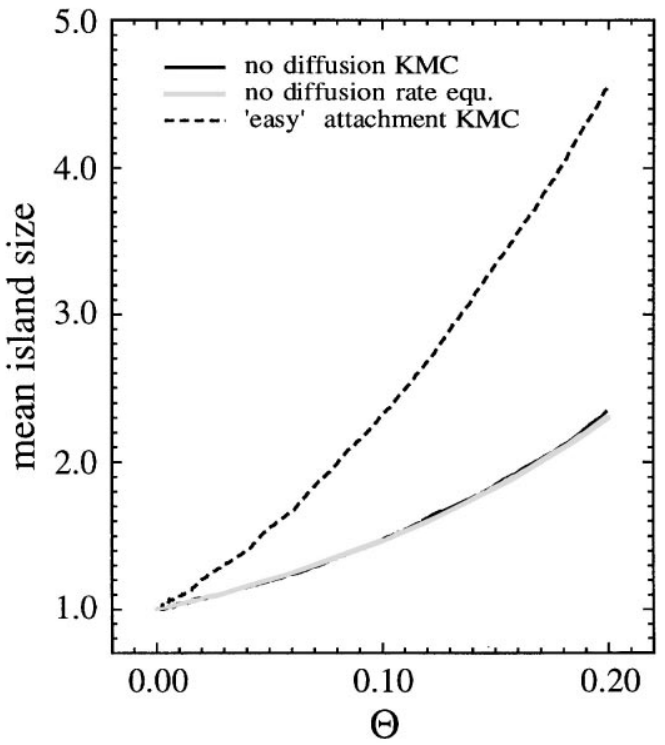

Fig. 14. The mean island size provides valuable information to investigate whether there is transient mobility and/or "easy" attachment to islands. (a) Deposition of $\mathrm{Ag}$ onto $\mathrm{Pt}\left(\begin{array}{lll}1 & 1 & 1\end{array}\right)$ at $35 \mathrm{~K}$ where ordinary diffusion is frozen shows predominantly monomers imaged as bright dots of 4-7 $\AA$ width (their frizzyness is due to mechanical instabilities that were considerably reduced in an improved setup). Due to tip convolution monomers are not discerned from dimers. The mean island size amounts to $1.2 \pm 0.3$ atoms, clearly ruling out transient mobility by comparison with theoretical curves (b) discussed in the text (from Ref. [128]).

yield the same mean island size. Transient jumps of condensing atoms increase the mean island size only in those cases where they proceed towards other monomers or islands ending up with attachment. Transient mobility can be discerned from attachment as the first is non-thermal whereas the latter is assumed to be thermally activated. Therefore decreasing the deposition temperature is expected to freeze in "easy" attachment, whereas transient mobility, if there is any, should always appear.

The experimental value for the mean island size of $1.2 \pm 0.3$ atoms obtained for $\mathrm{Ag} / \mathrm{Pt}\left(\begin{array}{lll}1 & 1 & 1\end{array}\right)$ at $0.1 \mathrm{ML}$ deposited at $35 \mathrm{~K}$ clearly allows one to rule out transient mobility, as well as easy attachment over one or more sites. The mean size obtained at slightly higher temperatures, for example $2.8 \pm 0.4$ at $50 \mathrm{~K}$ and $0.12 \mathrm{ML}$ (see Fig. 8(a)), can be due to post-deposition mobility, easy attachment, or a combination of both, as discussed above. If we assume it to be predominantly caused by easy attachment over one site, we arrive at an estimate of about 70\% of terrace barrier for that process (attachment of monomers to islands is frozen in at $35 \mathrm{~K}$ and active at $50 \mathrm{~K}$ ) which is comparable to the EMT result employed for Fig. 13(c). These results are in agreement with the observations made for $\operatorname{Ir} / \operatorname{Ir}\left(\begin{array}{lll}1 & 1 & 1\end{array}\right)$ $[129,130]$ as far as attachment towards islands with a lower barrier is concerned. However, they are at variance concerning the degree to which the barrier is reduced. Wang and Ehrlich observe depletion zones at $20 \mathrm{~K}$, requiring the barrier to be reduced by one order of magnitude for the case of Ir. The results for $\mathrm{Ag} / \mathrm{Pt}\left(\begin{array}{lll}1 & 1 & 1)\end{array}\right.$ indicate a reduction by only $30 \%$ for the last $\mathrm{fcc} \rightarrow \mathrm{fcc}$ jump towards a neighbor. Besides electronic reasons distinct to the elements, this difference may be due to the different cluster sizes involved in the two experiments: for $\mathrm{Ag} / \mathrm{Pt}$ principally monomers and dimers were 
involved, whereas for Ir/Ir attachment towards larger clusters comprising from 12 to 59 atoms was studied.

Transient mobility has frequently been addressed in the literature since the early days of FIM investigations of diffusion [137-139]. It is often invoked to explain experimental observations, such as 2D growth at low temperature [140], although up to now direct experimental proof for transient mobility does not exist for metal on metal condensation ${ }^{17}$ (see overview given in Ref. [144]). Very often, alternative explanations evolve which rationalize the observed behavior without the assumption of transient mobility [145]. The experiments described above allow examination of the issue of transient mobility for a wide range of systems and similarly enable details to be learnt concerning monomer attachment to other monomers or islands.

In this section we have seen that the scaling laws of classical nucleation theory are valid only when nucleation takes place solely during deposition, which is the case for $D / F>10^{5}$. Below this diffusivity to flux range there are intrinsic deviations from scaling which are accompanied by post-deposition mobility when the substrate is investigated at the temperature of and at some time after deposition. With decreasing deposition temperatures, diffusion becomes slow with respect to deposition leading first to island growth after deposition, second to nucleation taking place after deposition, and finally to statistic growth in the extreme case of no thermal mobility. It was shown how these various low temperature growth phenomena can be understood quantitatively, and how island densities collected at low temperatures can be analyzed to gain additional information, for example on attachment to islands and transient mobility for an epitaxial system. Such effects are details which, although similarly appearing at higher temperatures, only affect island densities and morphologies at very low temperatures $\left(D / F<10^{5}\right)$.

\subsection{Effect of isotropic strain on surface diffusion}

Isotropic strain, as it appears in the growth of pseudomorphic heteroepitaxial layers, is the variation of the in-plane lattice constant of the film material with respect to its bulk value. ${ }^{18}$ Theorists have pointed out several times that surface diffusion should be strongly affected by strain. Two molecular dynamics (MD) simulations proposed that for semiconductor surfaces strain changes adatom mobilities [146,147]. A theoretical study explicitly investigated the effect of strain on the nucleation kinetics on vicinal surfaces [148]. There was even the suggestion to purposely apply strain in order to influence the growth kinetics, the goal being to grow linear structures attached to the steps of a vicinal substrate [149]. It is only very recently, however, that the possibility of experimentally addressing the influence of isotropic strain on surface diffusion has risen. Access to diffusion on strained surfaces was achieved with the nucleation method outlined in Section 3.1 for the example of $\operatorname{Ag} / \operatorname{Pt}\left(\begin{array}{lll}1 & 1\end{array}\right)$. The experiments showed that the barrier to surface migration on a pseudomorphic Ag monolayer on $\operatorname{Pt}(111)$, which is under compressive strain, is significantly reduced with respect to that on a fully relaxed $\operatorname{Ag}\left(\begin{array}{lll}1 & 1 & 1\end{array}\right)$ surface [150]. This observation prompts the question whether this effect is indeed due to strain, or

\footnotetext{
17 "Hot" species that can be created during the course of dissociative adsorption of a molecule are not under consideration here [141-143].

${ }^{18}$ The misfit $m=(b-a) / a$, on the other hand, relates the natural lattice constant of the film material $b$ to that of the substrate $a$. Consequently, strain $\varepsilon$ and misfit $m$ have different sign and also slightly different numbers, e.g., for Ag grown on Pt $\varepsilon=-4.15 \%$ and $m=+4.33 \%$.
} 
a)

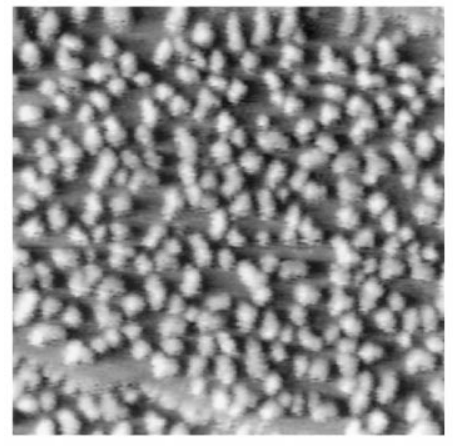

b)

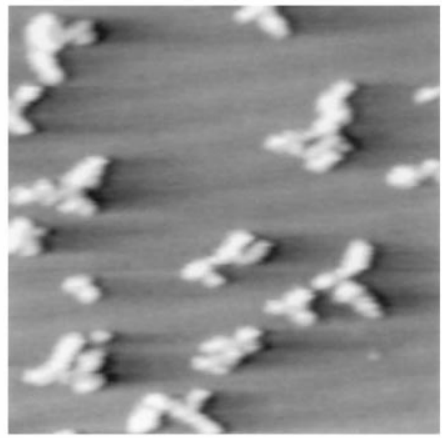

d) c)

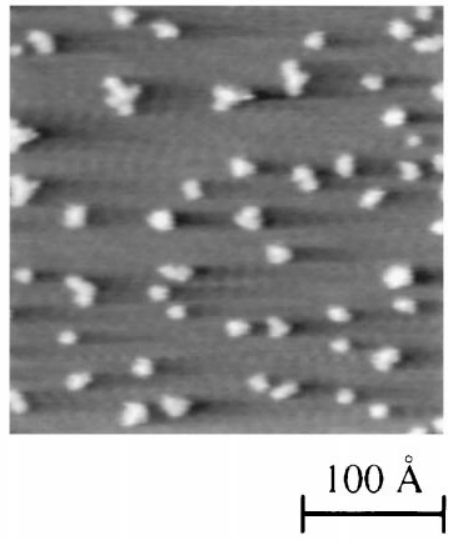

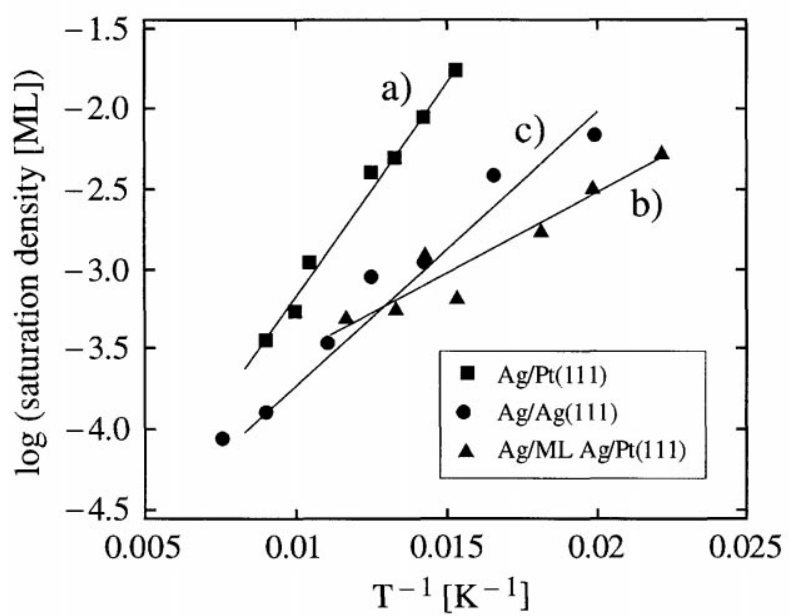

Fig. 15. STM images showing the nucleation of submonolayer coverages of $\mathrm{Ag}$ on $\operatorname{Pt}\left(\begin{array}{lll}1 & 1 & 1\end{array}\right)$ (a), on $1 \mathrm{ML} \mathrm{Ag} / \operatorname{Pt}\left(\begin{array}{lll}1 & 1 & 1\end{array}\right)$ (b), and on $\operatorname{Ag}(111)$ (c) ((a) $T=65 \mathrm{~K}$; (b) $T=65 \mathrm{~K}$; and (c) $T=60 \mathrm{~K}$ ). (d) Arrhenius plot of saturation island densities $(\Theta=0.12 \mathrm{ML})$ derived from STM for nucleation of $\mathrm{Ag}$ on $\mathrm{Pt}\left(\begin{array}{lll}1 & 1 & 1\end{array}\right)(\boldsymbol{\square})$, on $1 \mathrm{ML} \mathrm{Ag}$ adsorbed on $\mathrm{Pt}\left(\begin{array}{lll}1 & 1 & 1\end{array}\right)(\boldsymbol{\Delta})$, and on $\operatorname{Ag}\left(\begin{array}{lll}1 & 1 & 1\end{array}\right)(\bigcirc)$, respectively (from Ref. [150]).

rather caused by the electronic adlayer-substrate coupling. In this section, we discuss these first experimental results on surface diffusion on isotropically strained surfaces in light of new theoretical results from ab initio calculations. For a more detailed survey of the effect of strain on surface diffusion, including also interlayer diffusion, the reader is referred to Ref. [29].

For the system $\mathrm{Ag} / \mathrm{Pt}\left(\begin{array}{lll}1 & 1 & 1\end{array}\right)$ there is a strong layer dependence of island densities ${ }^{19}$ as becomes evident from inspection of Fig. 15. The three STM images were obtained for low temperature nucleation of $\mathrm{Ag}$ on $\operatorname{Pt}(111)$, of $\mathrm{Ag}$ on the first $\mathrm{Ag}$ monolayer grown on $\operatorname{Pt}(111)$, and of $\mathrm{Ag}$ on

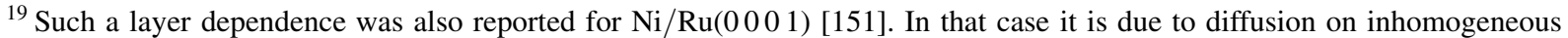
surfaces, where strain is relieved in moiré or dislocation patterns. Nucleation on inhomogeneous substrates will be addressed in Section 4.2.
} 
$\mathrm{Ag}(111$ ), respectively. The lowest island density is observed in Fig. 15(b), i.e. for Ag growth on the Ag monolayer. The structure of the $\mathrm{Ag}$ monolayer is pseudomorphic ${ }^{20}$ with respect to the $\operatorname{Pt}\left(\begin{array}{lll}1 & 1 & 1\end{array}\right)$ substrate [154,155] and continues its fcc stacking [156]. Due to the lattice mismatch between Ag and $\mathrm{Pt}$, the interatomic distance in this layer is reduced by $4.15 \%$ with respect to the $\mathrm{Ag}$ bulk value.

The temperature dependence of the saturation island densities strongly differs on these three substrates, indicative of differing migration barriers. Nucleation on the pseudomorphic Ag monolayer is characterized by the smallest slope in the Arrhenius plot represented in Fig. 15(d), followed by $\operatorname{Ag}\left(\begin{array}{lll}1 & 1 & 1\end{array}\right)$, and finally by $\operatorname{Pt}\left(\begin{array}{lll}1 & 1 & 1\end{array}\right)$. These slopes directly yield the parameters for surface diffusion on these layers, as dimers are stable for the data shown here. This is indicated by the absence of changes in the slope in the Arrhenius plot; it has also been verified by the onset of Ostwald ripening for dimers [157], analogous to the example of $\mathrm{Ag} / \mathrm{Pt}$ discussed above. The resulting migration barriers, $E_{\mathrm{m}}$, on the different isotropic layers are $168 \pm 5 \mathrm{meV}$ for $\mathrm{Ag} / \mathrm{Pt}$ (see Section 3.1), $60 \pm 10 \mathrm{meV}$ for the pseudomorphic Ag layer, and $97 \pm 10 \mathrm{meV}$ for $\mathrm{Ag}$ diffusion on a strain-free $\mathrm{Ag}(1111)$ surface [150].

The most conspicuous effect uncovered by the STM experiment is the low barrier for Ag diffusion on the pseudomorphic Ag layer compared to unstrained $\operatorname{Ag}(111)$. Two effects are conceivable causes of the observed lowering. It may either be due to compressive strain or to electronic effects caused by the underlying Pt substrate. Calculations within EMT [150] and ab initio density functional theory (DFT) calculations [158] both clearly indicate that strain is the origin for the reduction of the diffusion barrier. The results obtained for diffusion of $\mathrm{Ag}$ on a $\operatorname{Ag}\left(\begin{array}{lll}1 & 1 & 1\end{array}\right)$ slab as a function of its lattice constant are presented in Fig. 16. Both EMT and DFT calculations clearly show that strain has a pronounced influence on migration barriers. While the EMT calculations capture the main effect of a linear dependence of $E_{\mathrm{m}}$ for small variations of the lattice constant, absolute values are underestimated. This is a general difficulty of EMT for diffusion on close-packed surfaces [126,134]. In addition, deviations

a)

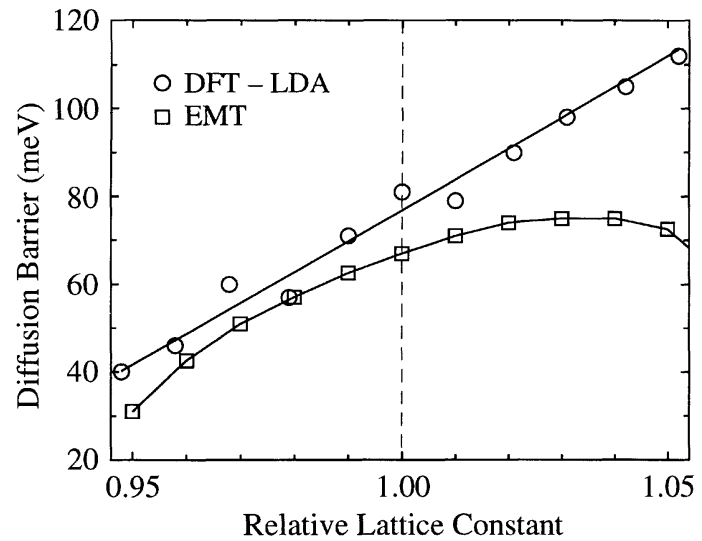

b)

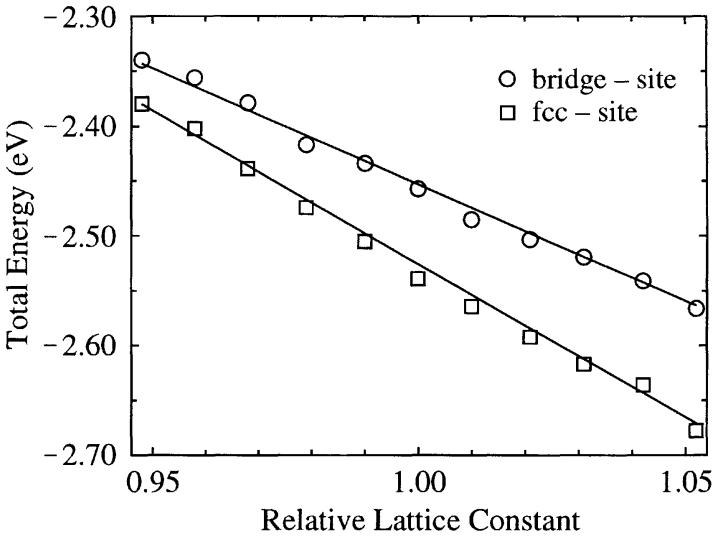

Fig. 16. (a) Diffusion barrier for $\mathrm{Ag}$ self-diffusion on $\operatorname{Ag}\left(\begin{array}{lll}1 & 1 & 1\end{array}\right)$ as a function of the relative lattice constant. The lattice constant is normalized to the computed bulk lattice constants of Ag, $a=4.05 \AA$ (DFT) and $4.075 \AA$ (EMT). (b) Binding energies in the transition (bridge) and binding (fcc-hollow) sites as a function of tensile or compressive strain (from Ref. [158]).

\footnotetext{
${ }^{20}$ Note that dislocations form in submonolayer islands which reach a critical size $[152,153]$. These dislocations then disappear when second layer islands provide a 2D gas phase on top of the first layer driving the system back to pseudomorphic [152]. For the present context it suffices to know that a completed first monolayer is always pseudomorphic.
} 
from linearity become apparent at the lattice constant extremes examined. The ab initio DFT results, on the other hand, show a linear behavior of $E_{\mathrm{m}}$, with a slope of $\partial E_{\mathrm{m}} / \partial \varepsilon=7 \mathrm{meV}$ per percent of change over the entire range of lattice constants studied. This predicts a reduction of $E_{\mathrm{m}}$ by $30 \mathrm{meV}$ induced by $4.15 \%$ compressive strain, in very good agreement with the experimental finding. Also absolute numbers agree reasonably well with experimental values: $E_{\mathrm{m}}=81 \mathrm{meV}$ for diffusion on $\operatorname{Ag}\left(\begin{array}{lll}1 & 1 & 1\end{array}\right)$ with its equilibrium lattice constant obtained from LDA, and $60 \mathrm{meV}$ on $\mathrm{Ag}\left(\begin{array}{lll}1 & 1 & 1\end{array}\right)$ compressed to the Pt lattice constant. The migration barrier for $\mathrm{Ag}$ on a pseudomorphic $\mathrm{Ag}$ monolayer on $\mathrm{Pt}(111)$ is found to be $63 \mathrm{meV}$ which is identical within the error margin of the calculations to the strained $\operatorname{Ag}\left(\begin{array}{lll}1 & 1 & 1\end{array}\right)$ case [158]. Therefore, strain is identified as origin for the observed lowering of the migration barrier. The strain effect is essentially independent of electronic contributions of the substrate underneath.

The physical reason for the strong influence of strain on diffusion is the different effect the lattice constant has on the binding energies of an adatom in the bridge, compared to the hollow site. The binding energies in both sites show an approximately linear dependence on strain, as evident from inspection of Fig. 16(b). Binding to the surface becomes less strong when the substrate is compressed. This can be understood in a simple electron density picture. A system composed of a surface with a high electron density gains less energy in adsorbing an adatom than one where the surface electron density is low. The slope of the binding energy is larger at the three-fold coordinated fcc hollow site than at the two-fold bridge site. The bridge site becomes less affected by changes of the electron density at the surface as there the adatom is located further away from the surface. As a consequence the migration barrier $E_{\mathrm{m}}$, which denotes the difference between the binding energies at the bridge and hollow site, strongly varies with lattice constant. It increases for lattice expansion and decreases for compressive strain.

So far, the system provided by $\mathrm{Ag} / \mathrm{ML} \mathrm{Ag} / \mathrm{Pt}\left(\begin{array}{lll}1 & 1 & 1)\end{array}\right)$ and $\mathrm{Ag} / \mathrm{Ag}\left(\begin{array}{lll}1 & 1 & 1\end{array}\right)$ has given the only quantitative experimental result on the effect of strain on surface diffusion. From several recent calculations motivated by these experimental findings, it can be expected that isotropic strain generally has a pronounced influence on adatom mobilities. Ab initio calculations performed for self-diffusion on fcc(1 11 1) surfaces of several metals $(\mathrm{Ni}, \mathrm{Pd}, \mathrm{Pt}, \mathrm{Cu}, \mathrm{Ag}, \mathrm{Au})$ predict tendencies very similar to those

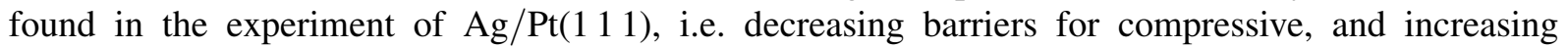
barriers for tensile strain [159]. The effect of strain on diffusion on an fcc(100) surface has been addressed in a recent DFT calculation [121]. For self-diffusion on $\operatorname{Ag}(100)$, the authors find that compressive strain decreases the barrier for hopping diffusion, in line with the behavior on closepacked surfaces. However, larger lattice constants were reported to reduce the barrier for exchange diffusion, implying a strain induced transition in diffusion mechanism. A calculation with pair interactions modeled by Lennard-Jones potentials shows a linear dependence of the migration barrier

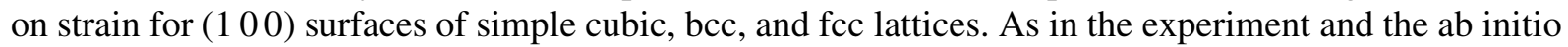
calculations for fcc(1 111$)$ surfaces, $\partial E_{\mathrm{m}} / \partial \varepsilon>0$ [160].

All theoretical studies agree that strain has a pronounced effect on surface diffusion. While it can be expected that diffusion on close-packed surfaces follows the experimental observations reported in the first part of this section, smaller lattice constants reducing barriers and vice versa, the situation on square lattices can become complicated by a transition to exchange diffusion. Effects due to strain provide great potential for further study. Such experiments are feasible with the nucleation method described in Section 2.

In order to underline the importance of the apparent link of strain to surface diffusion, we mention a recent example: self-organization in the growth of quantum-dot superlattices. Typically, such 
superlattices are grown sequentially, for example on a GaAs substrate one grows strained InAs islands which are then capped with GaAs. This sequence is repeated yielding layers of InAs dots embedded into GaAs. These systems have the fascinating feature that island sizes and spacings become more regular as the number of such sandwich layers is increased. Present models to explain this high degree of self-ordering incorporate a higher nucleation probability on top of buried islands as essential ingredient [161]. The physical reason can be strain reducing the diffusivity in these areas which would yield a higher local density of monomers. ${ }^{21}$ As $n_{1}$ enters quadratically in the nucleation rate (and even to higher order for $i>1$ ) this explains the higher nucleation probability on top of buried islands. This example of self-ordering, possibly mediated by the interplay of strain and diffusion, may have practical importance. It provides a mechanism for creating the uniform island sizes required to make use of quantum size effects in electronic devices.

We further note that in general, every long range elastic interaction, for example around steps, will have direct impact on diffusion and nucleation. ${ }^{22}$ In this context it is important to notice that isotropic strain not only affects intralayer but also strongly influences interlayer diffusion [163,164]. The nucleation and growth kinetics of heteroepitaxial systems may therefore be dominated by strain effects.

\subsection{Energy barriers and attempt frequencies from nucleation densities}

The observation of island densities at temperatures where dimers are stable opened up a new method for the measurement of barriers and attempt frequencies for single adatom diffusion. This nucleation method is a complement of conventional techniques, such as FIM or fluctuation methods (mostly based on FEM), which were previously our most important and precise sources for the determination of activation energies of diffusing particles on surfaces. The classical methods have certain drawbacks that can be overcome by the nucleation method. FIM is restricted to materials with a high mechanical strength. Fluctuation methods need a constant average density of diffusing particles, of which the local fluctuations are then recorded. Therefore, irreversible cluster creation has to be avoided as it would steadily decrease the overall density. The particles must either interact repulsively or fluctuation experiments have to be carried out at temperatures and pressures where there is an equilibrium between 2D gas and 2D solid. As far as epitaxial systems are concerned, the particles interact attractively, and they diffuse much too fast at conditions where clusters begin to break up. This hampers accessing them with fluctuation methods. The nucleation method does not have these restrictions which renders it a rather valuable tool for future studies of surface diffusion.

The nucleation method presented here differs from previous nucleation studies performed with EM [11] insofar as the $i=1$ regime can be addressed in situ for any system, either in real space by VT-STM or with diffraction techniques. ${ }^{23}$ There are many recent studies of surface diffusion relying on the nucleation method. In this section we will discuss the examples for metal surfaces in comparison with

\footnotetext{
${ }^{21}$ Superlattices of coherently strained $\mathrm{Si}_{0.25} \mathrm{Ge}_{0.75}$ islands buried in $\mathrm{Si}(100)$ spacer layers also show this self-ordering [162]. As Ge has the larger lattice constant, the Si spacer very likely has a slightly larger lattice constant on top of a buried GeSi island. According to the results reported in this section this would reduce diffusion as required.

${ }^{22}$ An example is the local variation of mobility expected on top of heteroepitaxial islands [160].

${ }^{23}$ Diffraction techniques may in certain cases be hampered by the presence of steps and the more indirect information on island densities. However, they provide better statistics.
} 
FIM experiments and theoretical calculations with the goal of drawing conclusions on the validity and limits of the nucleation method. We will also address a lowering of attempt frequencies so far systematically observed for systems with migration barriers below $100 \mathrm{meV}$.

\subsubsection{Surfaces with square symmetry}

Table 2 summarizes the barriers for single atom migration as obtained from measurements of nucleation island densities for various metal surfaces in comparison with theoretical calculations and FIM values, where available. One of the first systematic STM studies of the variation of island densities with temperature was reported by Stroscio and Pierce for Fe(100) homoepitaxy [86]. Although the nucleation of Fe was studied above room temperature, using size distributions the authors could verify that they were in the irreversible growth regime up to $530 \mathrm{~K}$ [75]. Accordingly the island densities below that temperature showed Arrhenius behavior from which the activation energy of Fe diffusion on

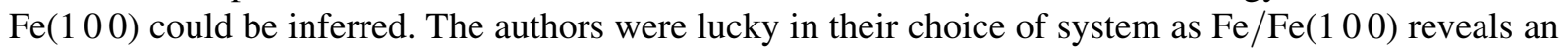
exceptionally strong dimer bond which extends the $i=1$ regime far above room temperature. For comparable systems, dimer instability typically appears at much lower temperatures, e.g. $320 \mathrm{~K}$ for $\mathrm{Cu} / \mathrm{Ni}\left(\begin{array}{lll}1 & 0 & 0)\end{array}\right.$ [77] and $223 \mathrm{~K}$ for $\mathrm{Cu} / \mathrm{Cu}\left(\begin{array}{lll}1 & 0 & 0\end{array}\right)[95,165]$.

For $\mathrm{Cu}\left(\begin{array}{lll}1 & 0 & 0\end{array}\right)$ homoepitaxy, there are several studies reporting identical values for $E_{\mathrm{m}}$ [95,165-167]. As they emerge from quite different experimental techniques and principles of analysis, this agreement is encouraging. The only result that has long been considered as a discrepancy [168] was a much too low value of $0.28 \pm 0.06 \mathrm{eV}$ reported in a He-diffraction study that applied the nucleation method [96]. The reason for this apparent disagreement is the scaling exponent used by the authors in their analysis. They quote that their experiment is sensitive only to very large islands formed in the immediate beginning of deposition. These are quoted to scale with $\chi=\frac{1}{2}$, but from the KMC-results discussed in Section 2 (see Fig. 4(b) and also Ref. [85]) it is evident that for very low coverages $\chi$ is much closer to $\frac{1}{3}$, even falling slightly below this value. In addition, at $0.5 \mathrm{ML}$ all islands are very large suggesting that at that stage, the side bands in the He-diffraction scans reflect the total island density. When repeating the analysis with $\chi=\frac{1}{3}$, the data by Ernst et al. are fully consistent with the existing experimental work. ${ }^{24}$ It should be noted that also the SPA-LEED data of Zuo et al. were re-interpreted [169], which left $E_{\mathrm{m}}$ basically unchanged $(0.38 \mathrm{eV})$, but yielded a more reasonable value for the dimer bond energy $(0.20 \mathrm{eV})$ than that of the original paper $(0.06 \mathrm{eV})$.

Self-diffusion on $\operatorname{Ag}\left(\begin{array}{lll}1 & 0 & 0\end{array}\right)$ has been investigated experimentally using low energy ion scattering (LEIS) [170] and STM [87]. In the STM study island densities were measured in the temperature regime of $295-370 \mathrm{~K}$ where coarsening happens for that system. This was accounted for in KMC simulations, nevertheless it introduces the dimer bond energy as additional fit parameter. The limited temperature range addressed further forced the assumption of an attempt frequency rather than its measurement. In light of these limitations, the error bars given by the authors, and reproduced in Table 2, appear rather optimistic. The LEIS study determined the onset temperature of monomer diffusion and similarly had to assume the attempt frequency. Uncertainties about its value have been included by the authors in the error bars.

The diffusion parameters for $\mathrm{Cu} / \mathrm{Ni}\left(\begin{array}{lll}1 & 0 & 0\end{array}\right)$ could be obtained quite precisely from an STM study where the $i=1$ regime was explicitly verified and a large range of $D / F$ addressed $[77,171]$. In

${ }^{24}$ The experiment of Ernst et al. was also re-interpreted with a similar result in Ref. [81]. 
Table 2

Energy barriers for metal diffusion on isotropic metal surfaces determined from nucleation experiments

\begin{tabular}{|c|c|c|c|c|c|}
\hline System & Experiment $E_{\mathrm{m}}(\mathrm{eV})$ & Exp. technique & Type of analysis & Theory $E_{\mathrm{m}}(\mathrm{eV})$ & Type of calculation \\
\hline $\mathrm{Fe} / \mathrm{Fe}\left(\begin{array}{lll}1 & 0 & 0\end{array}\right)$ & $0.45 \pm 0.05[86]$ & STM & $\begin{array}{l}i=1 \text { at } T<530 \mathrm{~K} \\
\text { via island sizes [75] }\end{array}$ & & \\
\hline \multirow[t]{4}{*}{$\mathrm{Cu} / \mathrm{Cu}\left(\begin{array}{lll}1 & 0 & 0\end{array}\right)$} & $0.42 \pm 0.09^{\mathrm{a}}[96]$ & He-diffraction & Island distances at $0.5 \mathrm{ML}$ & $\begin{array}{l}0.48 \text { at } \\
260-450 \mathrm{~K}[47]\end{array}$ & $\begin{array}{l}\text { EAM, including } \\
\text { phonon contrib. }\end{array}$ \\
\hline & $0.36 \pm 0.03[95]$ & SPA-LEED & Island distances at $0.3 \mathrm{ML}$ & $\begin{array}{l}0.43[46] \\
0.43[168] \\
0.43[176]\end{array}$ & $\begin{array}{l}\text { MD-N-Body-Pot. } \\
\text { MD-CEM } \\
\text { EMT }\end{array}$ \\
\hline & $0.39 \pm 0.06[167]$ & LEIS & Diffusion onset at $140 \pm 5 \mathrm{~K}$ & $0.38 / 0.53[177]$ & $\begin{array}{l}\text { EAM: AFW/VC } \\
\text { Potentials }\end{array}$ \\
\hline & $0.40[166]$ & He-scattering & Stationary step densities & $0.45[178]$ & EAM \\
\hline $\mathrm{Ag} / \operatorname{Ag}\left(\begin{array}{lll}1 & 0 & 0\end{array}\right)$ & $\begin{array}{l}0.40 \pm 0.05[170] \\
0.330 \pm 0.005[87]\end{array}$ & $\begin{array}{l}\text { LEIS-TOF } \\
\text { STM }\end{array}$ & $\begin{array}{l}\text { Diffusion onset at } 160 \pm 5 \mathrm{~K} \\
n_{x} \text { at } 295-370 \mathrm{~K}, i \geq 1\end{array}$ & $\begin{array}{l}0.50 \pm 0.03[179] \\
0.25 \pm 0.02[45] \\
0.48[180] \\
0.365[114] \\
0.48[177]\end{array}$ & $\begin{array}{l}\text { ab initio FP-LMTO } \\
\text { MD/MC-CEM } \\
\text { MD-EAM } \\
\text { EMT } \\
\text { EAM }\end{array}$ \\
\hline $\mathrm{Cu} / \mathrm{Ni}\left(\begin{array}{lll}1 & 0 & 0\end{array}\right)$ & $\begin{array}{l}0.36 \pm 0.02[77] \\
0.41 \pm 0.07[172]\end{array}$ & $\begin{array}{l}\text { STM } \\
\text { SPA-LEED }\end{array}$ & $\begin{array}{l}n_{x}(T), i=1 \text { via } n_{x}(F) \text { and sizes } \\
\text { Post deposition mobility }\end{array}$ & $\begin{array}{l}0.47[77] \\
0.45[181]\end{array}$ & $\begin{array}{l}\text { EMT } \\
\text { MD-CEM }\end{array}$ \\
\hline $\mathrm{Ag} / \operatorname{Pt}\left(\begin{array}{lll}1 & 1 & 1\end{array}\right)$ & $0.168 \pm 0.005[59]$ & STM & $\begin{array}{l}n_{x}(T), i=1 \text { at } T \leq 110 \mathrm{~K} \\
\text { via onset of Ostwald ripening }\end{array}$ & $\begin{array}{l}0.20[182] \\
0.15[158]\end{array}$ & $\begin{array}{l}\text { ab initio FP-LDA } \\
\text { ab initio DFT-LDA }\end{array}$ \\
\hline 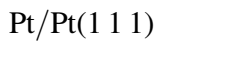 & $\begin{array}{l}0.26 \pm 0.01[37] \\
0.25 \pm 0.02[126]\end{array}$ & $\begin{array}{l}\text { STM } \\
\text { FIM }\end{array}$ & $\begin{array}{l}n_{x}(T) \text { cf. KMC nuc.-curve meth. } \\
\text { Onset } T \text { of diffusion }\end{array}$ & $\begin{array}{l}0.39[159] \\
0.38[126]\end{array}$ & $\begin{array}{l}\text { ab initio DFT } \\
\text { ab initio }\end{array}$ \\
\hline \multirow[t]{3}{*}{$\mathrm{Ag} / \operatorname{Ag}\left(\begin{array}{lll}1 & 1 & 1\end{array}\right)$} & $0.097 \pm 0.010[150]$ & STM & $\begin{array}{l}n_{x}(T), i=1 \text { at } T \leq 130 \mathrm{~K} \\
\text { via onset of Ostwald ripening }\end{array}$ & $0.140 / 0.100$ [159] & $\begin{array}{l}\text { ab initio } \\
\text { DFT-LDA/GGA }\end{array}$ \\
\hline & $0.15 \pm 0.10[183]$ & $\begin{array}{l}\text { EM for } \mathrm{Ag} \text { on } \\
2 \mathrm{ML} \mathrm{Ag} / \mathrm{W}\left(\begin{array}{lll}1 & 1 & 0\end{array}\right)\end{array}$ & $n_{x}(T), i \gg 1$ since $T>450 \mathrm{~K}$ & $0.081[158]$ & ab initio DFT-LDA \\
\hline & $\begin{array}{l}0.051 \pm 0.024[184] \\
0.180[185]\end{array}$ & $\begin{array}{l}\text { He-scattering } \\
\text { SPA-LEED }\end{array}$ & $\begin{array}{l}n_{x}[T], \text { subsequent ann. to } 260 \mathrm{~K} \\
n_{x}[T], i=1 \text { assumed for } T<200 \mathrm{~K}\end{array}$ & $0.140[179]$ & ab initio \\
\hline $\mathrm{Ag} / \mathrm{Ag} / \mathrm{Pt}\left(\begin{array}{lll}1 & 1 & 1\end{array}\right)$ & $0.060 \pm 0.010[150]$ & STM & $\begin{array}{l}n_{x}(T), i=1 \text { at } T \leq 70 \mathrm{~K} \\
\text { via onset of Ostwald ripening }\end{array}$ & $0.063[158]$ & ab initio DFT-LDA \\
\hline $\begin{array}{l}\mathrm{Ag} / \operatorname{Ag}\left(\begin{array}{lll}1 & 1 & 1\end{array}\right) \\
\varepsilon=-4.15 \%\end{array}$ & & & & $\begin{array}{l}0.060 \partial E / \partial \varepsilon= \\
7 \mathrm{meV} / \%[159] \\
0.060 \partial E / \partial \varepsilon= \\
7 \mathrm{meV} / \%[158]\end{array}$ & $\begin{array}{l}\text { ab initio } \\
\text { DFT-LDA/GGA } \\
\text { ab initio DFT-LDA }\end{array}$ \\
\hline
\end{tabular}

Theoretical values are given for comparison. For close-packed surfaces EMT or EAM-calculations systematically underestimate absolute values of diffusion barriers, we therefore only included ab initio calculations for that surface orientation.

${ }^{a}$ Corrected value obtained from the data of Ernst et al. [96] when applying the appropriate scaling exponent of $\frac{1}{3} . \varepsilon$ stands for strain. 
additional experiments carried out for the same system, the decay of the monomer concentration during post-growth was monitored by analyzing the time dependent profile of the $(0,0)$ LEED spot [172]. The results of this quite different approach agree will with those obtained from the nucleation method.

In Table 2 diffusion barriers obtained from calculations using approximate potentials as embedded atom method (EAM), EMT and its derivative, corrected EMT (CEM) are also detailed. These numbers are seen to agree quite well with the experimental values for the case of fcc(100) surfaces. Such approximate potentials are particularly useful as they permit one to address rather large systems [173175]. The investigation of phonon contributions [47] as well as temperature induced changes in the diffusion mechanism [46] using these potentials are also possible.

\subsubsection{Close-packed surfaces}

For the close-packed surfaces listed in Table 2 VT-STM has allowed rather precise experimental values for $E_{\mathrm{m}}$ to be inferred through the measurement of nucleation island densities. These values not only allow quantitative predictions on growth to be made, they may also serve as input for comparison with theoretical calculations. Ab initio calculations have considerably progressed in the recent past, and they provide rather good absolute values for migration barriers on many close-packed surfaces. The experimental pinning down of migration barriers for more and more systems drives further improvement of $a b$ initio theory. One of the items that could be addressed is the approximations for the exchange correlation $(\mathrm{XC})$ functional. Here the debate is the extent to which the generalized gradient approximation (GGA) is an improvement over the local density approximation (LDA) $[121,158,159]$.

The surface diffusion barrier for $\mathrm{Ag} / \mathrm{Pt}\left(\begin{array}{ll}1 & 1\end{array}\right)$ was calculated by Feibelman to be $200 \mathrm{meV}$ [182], and recently by Ratsch and Scheffler to be $150 \mathrm{meV}$, both in good agreement with the experimental value $(168 \pm 5 \mathrm{meV})$. For $\operatorname{Pt}\left(\begin{array}{lll}1 & 1 & 1\end{array}\right)$ self-diffusion, the established experimental result is $0.26 \pm 0.01 \mathrm{eV}$. This has been obtained both from FIM measurements [126], and using the "nucleation curve method" discussed in Section 3.3 [37]. Such results obtained from two independent techniques permit the verification of new experimental techniques; in this case the nucleation method can be evaluated with respect to well established FIM experiments. ${ }^{25}$ For Pt, the theoretical values (380 meV [126], $390 \mathrm{meV}$ (LDA) and $420 \mathrm{meV}$ (GGA) [159]) lie considerably above those coming from experiment.

For $\mathrm{Ag}\left(\begin{array}{lll}1 & 1 & 1\end{array}\right)$ self-diffusion the former EM result (obtained for $\mathrm{Ag}$ diffusion on $\left.2 \mathrm{ML} \mathrm{Ag/W(1} 10\right)$ in Ref. [183]) gives already the right estimate. It was acquired at irreversible growth conditions thus involving a pair binding model with the energy for a lateral bond as additional parameter. A SPA-LEED study by Luo et al. [185] evaluated island densities for $\mathrm{Ag} / \mathrm{Ag}(111)$ from the temperature dependence of the spot profile, analogous to $\mathrm{Zou}$ et al. for $\mathrm{Cu}(100)$ [95,165]. Irreversible island formation was assumed up to $200 \mathrm{~K}$. However, for that system Ostwald ripening of dimers sets in at $130 \mathrm{~K}$ [150]. Therefore the value of Zuo et al. contains the dimer bond energy via Eq. (8) and is consequently too large. The temperature for dimer instability was correctly determined for that system in a He-scattering study [184]. However, in this study the sample was annealed to $260 \mathrm{~K}$ before

\footnotetext{
${ }^{25}$ The published FIM results for $\mathrm{Pt} / \mathrm{Pt}(1111)$ report the onset temperature for diffusion [126]. The value for the barrier therefore relies on the assumption of a "universal" prefactor. This system is currently being re-examined by FIM experiments which look at the mean square displacement as a function of temperature [186]. To provide further tests of the nucleation method, Michely et al. foresee an investigation of $\operatorname{Ir} / \operatorname{Ir}\left(\begin{array}{lll}1 & 1 & 1\end{array}\right)$ by means of STM [187], as for that system the FIM value is quite precise, $E_{\mathrm{m}}=267 \pm 4 \mathrm{meV}[117]$.
} 
inspection with the He beam. This causes considerable coarsening which might not be independent of the initial island size and therefore the results are obscured. ${ }^{26}$ The only experimental value with significant precision so far is that obtained in a low temperature nucleation experiment. This yields a surface diffusion barrier of $97 \pm 10 \mathrm{meV}[150,189]$. Theory yields rather good agreement with this value, $100 \mathrm{meV}$ was calculated by Mortensen et al. [159] with GGA. The LDA value of $81 \mathrm{meV}$ obtained by Ratsch et al. [158] is also in reasonable agreement with experiment. However, both studies disagree on the effect of GGA vs. LDA. Whereas Mortensen et al. report lower values with GGA ( $100 \mathrm{meV}$ vs. $140 \mathrm{meV}$ with LDA), Ratsch et al. [158] find that their results become only weakly affected by replacing LDA by GGA, in addition they report larger values for GGA. On the other hand, the large LDA value of $140 \mathrm{meV}$ reported by Mortensen et al. is consistent with the result of an LDA calculation by Boisvert et al. [179]. This comparison between different ab initio calculations for the case of $\mathrm{Ag}$ diffusion on $\operatorname{Ag}(1111)$ shows that there is still some controversy between the codes and assumptions used by various authors as they produce very different results ranging from $E_{\mathrm{m}}=140$ to $81 \mathrm{meV}$.

The effect of isotropic strain on diffusion barriers was addressed in two ab initio studies for $\operatorname{Ag}\left(\begin{array}{llll}1 & 1 & 1\end{array}\right)$ self-diffusion. These yield consistent results for the derivative of $E_{\mathrm{m}}$ with respect to strain $\varepsilon[158,159]$ and are in good agreement with experiment $\left(\partial E_{\mathrm{m}} / \partial \varepsilon=10 \mathrm{meV} / \%\right)$. Notice, Mortensen et al. infer their values by comparing calculations performed with the LDA lattice constant to those done with the GGA lattice constant which is by $1-3 \%$ larger. ${ }^{27}$ They found large values for $\partial E_{\mathrm{m}} / \partial \varepsilon$ for all transition metals investigated suggestive for strain generally having a strong effect on surface diffusion. Ratsch et al. explicitly varied the lattice constant and thus were able to show that $E_{\mathrm{m}}$ varies linearly with strain.

\subsubsection{Diffusion for systems with extremely small barriers}

Recent experiments addressing diffusion on fcc(llll 111$)$ surfaces seem to suggest that elements with very small barriers also have extremely small values for their prefactors. Whereas for all systems with barriers larger than $100 \mathrm{meV}$, prefactors close to the "universal" value in the range of typical phonon frequencies $10^{12}-10^{13} \mathrm{~s}^{-1}$ are reported, ${ }^{28}$ the prefactors found for systems with smaller barriers systematically decrease with decreasing $E_{\mathrm{m}} .{ }^{29}$ This decrease over several orders of magnitude thus partly compensates the decrease in activation energies leading to isokinetic temperatures (see for example crossing of lines fitted to island densities in the Arrhenius plot Fig. 15). Although these results only exist for a few systems so far, this observation requires an explanation.

Earlier this century, Meyer and Neldel [192] published a compensation law that was found to be common to a wide range of thermally activated processes in physics, chemistry and biology (see Ref. [193] and references therein). This law links the prefactor $\nu_{0}$ to the activation energy $\Delta E$ in the equation $v_{0}=\nu_{00} \exp \left(\Delta E / \Delta E_{0}\right)$. A lower activation energy is thus associated with a smaller prefactor,

\footnotetext{
${ }^{26}$ A similar procedure has also been applied to study $\mathrm{Cu}\left(\begin{array}{llll}1 & 1 & 1\end{array}\right)$ self-diffusion with He-scattering [188]. Also in this study coarsening might have been involved.

${ }^{27}$ From the effect of strain on diffusion, one would expect the calculation with smaller lattice constants to yield smaller barriers. However, the opposite is the case.

${ }^{28}$ Examples are $\nu_{0}=3 \times 10^{13 \pm 0.75} \mathrm{~s}^{-1}$ for $\mathrm{Ag} / \mathrm{Pt}(111)$ [85], $\nu_{0}=5 \times 10^{12 \pm 0.5} \mathrm{~s}^{-1}$ for self-diffusion on $\operatorname{Pt}\left(\begin{array}{lll}1 & 1 & 1\end{array}\right)$ [37], and $\nu_{0}=4 \times 10^{11 \pm 1} \mathrm{~s}^{-1}$ for $\mathrm{Cu} / \mathrm{Ni}\left(\begin{array}{lll}1 & 0 & 0)\end{array}\right.$ [77].

${ }^{29}$ For example $\nu_{0}=1 \times 10^{9 \pm 0.6} \mathrm{~s}^{-1}, E_{\mathrm{m}}=60 \pm 10 \mathrm{meV}$ for Ag diffusion on the strained pseudomorphic Ag monolayer on

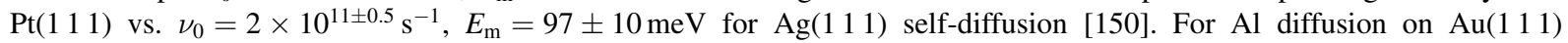
$\nu_{0}=10^{4 \pm 1} \mathrm{~s}^{-1}, E_{\mathrm{m}}=35 \pm 5 \mathrm{meV}$ [190], and finally for $\mathrm{Al}(111)$ we find $\nu_{0}=10^{7 \pm 1} \mathrm{~s}^{-1}, E_{\mathrm{m}}=42 \pm 5 \mathrm{meV}$ [191].
} 
which is exactly the situation observed in the diffusion measurements. In surface science, a similar compensation effect is known to appear in thermal desorption spectroscopy (TDS) [194]. There, mutual interaction of the desorbing species leads to a coverage dependence of the desorption barrier accompanied by a compensating variation of the prefactor. However, as this kind of compensation effect is mediated by coverage it is less closely related to the observations discussed here. Nevertheless it has significant consequences for adequate interpretation of TDS data [195]. The true analog to the observations addressed here is a compensation effect reported for bulk diffusion [196]. Recently, a compensation effect was also published for surface diffusion [193]. Based on MD calculations, the authors find that the prefactors for self-diffusion of 10 different metal systems lie on a straight line in a plot of $\ln \left(\nu_{0}\right)$ vs. $E_{\mathrm{m}}$, thus obeying the Meyer-Neldel law (with $E_{0}=190 \mathrm{meV}$, close to the value known from bulk diffusion). For activation energies between 0.13 and $1.3 \mathrm{eV}$ attempt frequencies were found to vary by a factor of 2000 around the "universal" value. However, the experimental observation of extremely small prefactors discussed above are orders of magnitude beyond the effect found by the authors of Ref. [193]. These small prefactors are hardly rationalized as the continuation of such a compensation effect to smaller activation energies.

There are several alternative explanations which emerge from theoretical studies using MD. Up to now, we have tacitly assumed that diffusion is well described by transition state theory (TST) in terms of jumps over a distance of one lattice site only and with the atoms being most of the time in binding sites giving them enough time for thermal equilibration with the substrate (rare event assumption of TST). There are, however, limits where these assumptions may no longer hold. Voter and Doll [197] showed that simple TST is valid for $T \lesssim \frac{1}{3 k} E_{\mathrm{m}}$. Sanders and DePristo noticed that the diffusion barrier measured in experiments for close-packed metals can deviate considerably from the corrugation of the potential energy surface. These effects appear at $T \approx \frac{1}{k} E_{\mathrm{m}}$ and are caused by recrossing, correlated long jumps (see also Refs. [180,198]), and not sufficient equilibration time between successive jumps [199], all violating the assumptions of TST. The nucleation experiments in question are typically performed at $T<110 \mathrm{~K}(k T \approx 10 \mathrm{meV})$. According to these MD studies, therefore they should still be well described by TST. Nevertheless it is not excluded that the effects found in these MD studies set in earlier in reality, and diffusion with extremely low barriers is missed by the traditional description.

A further feasible explanation comes from possible interactions between diffusing monomers. In order to influence nucleation, however, these would have to be of extremely long range (about 30 atomic distances), as monomer densities in the nucleation phase typically stay well below $10^{-3}$ atoms per site. The pair interactions reported so far are well below that range. Interactions ranging up to third nearest neighbors could be detected for several metal-pairs ( Re-Pd, W-Pd, Ir-Ir, and Re-Re) adsorbed on W(1 1 10$)[42,43,200]$. If pair interactions were to play a role, the measured diffusion coefficient would be a chemical one and lie below the tracer diffusion coefficient. ${ }^{30}$ The effect would lead to smaller apparent prefactors and therefore go into the right direction. Presently known pair interactions have much too short range to be the cause of the small prefactors. It is much more reasonable that the description of diffusion within transition state theory is no more valid for the systems with very small barriers. As the measurements are carried out at temperatures close to the diffusion barrier a description within diffusion theory of liquids is presumably more appropriate.

\footnotetext{
${ }^{30}$ Reduced diffusion coefficients and larger island densities due to repulsive interactions is one possible explanation of how surfactants work [201].
} 
In conclusion, the barriers for adatom diffusion obtained so far with the nucleation method for single crystal metal surfaces, agree rather well with other experiments where adatoms can be traced (FIM) or their attachment to islands and steps is followed (LEIS, SPA-LEED). The careful analysis of island densities, either by means of nucleation theory or KMC, has been discussed extensively above. This provides reliable results for the surface diffusion parameters ${ }^{31}$ which can reach the precision of state of the art FIM measurements. This encourages future applications of the nucleation method, carrying several advantages. It can be applied to non-perfect surfaces as they appear in heteroepitaxial growth, i.e. isotropically strained or dislocated substrates (examples for the latter will be discussed in Section 4.2). The method applies to attractively interacting particles that otherwise are hard to address by fluctuation or diffusion profile methods. Hence for epitaxial systems many future examples providing accurate experimental values are expected. From the theoretical side, the expected arrival of values for free electron metals is eagerly anticipated. Future studies focusing on attempt frequencies for diffusion will help to derive an adequate description of diffusion for systems with very small activation energies.

\section{Nucleation on anisotropic and patterned substrates}

In the present section we describe experiments and models of nucleation on anisotropic and patterned substrates. Anisotropic substrates have orientation dependent diffusion barriers and/or lateral bond energies. Under patterned substrates we understand reconstructed surfaces such as Au(111). Such substrates are inhomogeneous as the diffusion and adsorption energies vary upon translation. They can reveal complicated potential energy surfaces that can be exploited for self-organized growth of equally spaced (and sized) islands.

\subsection{Nucleation on anisotropic substrates}

For an fcc lattice, there are two types of low index surfaces that are anisotropic. The (1 10$)$ face reveals atomic troughs, 1 or 2 bulk lattice constants wide, depending on whether the surface is reconstructed in its clean state. The atoms along the troughs are arranged in close-packed rows. The strong anisotropy in interatomic distances has two consequences, weak interaction across the troughs and fast diffusion parallel to the troughs. The second type of anisotropic fcc surface is the hexreconstructed (100) surface, of which $\mathrm{Au}$ and $\mathrm{Pt}$ are well-known examples. These surfaces are characterized by large unit cells formed by quasi-hexagonal overlayers in which the interatomic distances are anisotropic due to incommensuration with the underlying square substrate. Here the anisotropy is weak as the distances vary only slightly. Nucleation studies performed on both kinds of surfaces show direct signatures of the substrate anisotropy in terms of island shapes and distances. We will briefly review these results. As in the case of isotropic substrates, we focus on systems that show no intermixing. ${ }^{32}$

\footnotetext{
${ }^{31}$ It is evident that reliable results are only obtained when studying the temperature dependence of island densities while establishing that the $i=1$ condition is maintained for the whole temperature range addressed.

${ }^{32}$ Metal systems that show surface alloy formation have been reviewed by Besenbacher et al. [28].
} 


\subsubsection{Sticking anisotropy on $f c c(110)$}

The first example where the formation of elongated islands was reported is $\mathrm{Si} / \mathrm{Si}(100)$. This surface reveals a dimer row reconstruction which leads to highly anisotropic diffusion, faster along the dimer rows [202]. It is therefore surprising that islands grow with an elongation perpendicular to this direction, namely along the dimer axis. However, as evidenced by annealing experiments which reveal a transition to compact islands, this shape is clearly not the energetically favored one [202]. The 1D shape was tentatively attributed to the sticking probability being higher to the island ends than to their sides [76,203]. This explanation could later be verified in an STM study which shows anisotropic sticking with a ratio of 50:1, overwhelming the diffusion anisotropy [204]. The in situ growth

a) $\mathrm{T}=265 \mathrm{~K}$

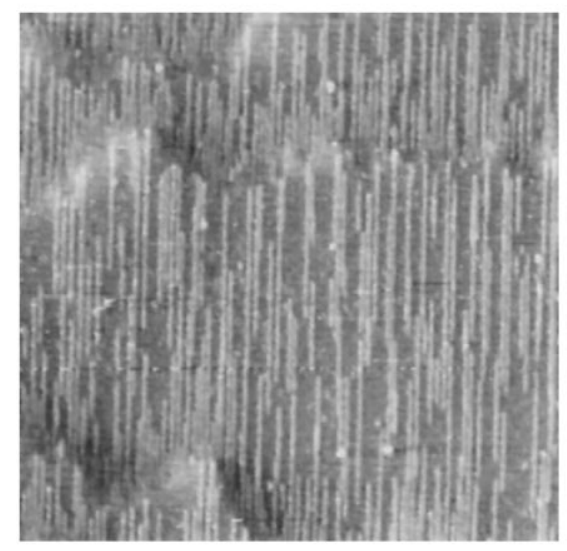

c) $\mathrm{T}=320 \mathrm{~K}$

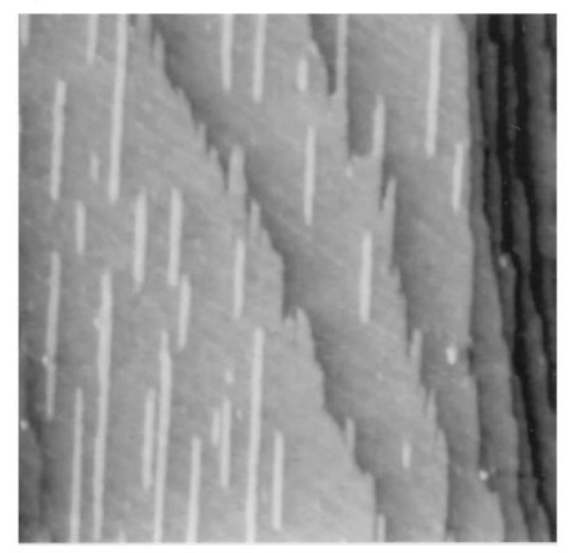

b) $\mathrm{T}=300 \mathrm{~K}$

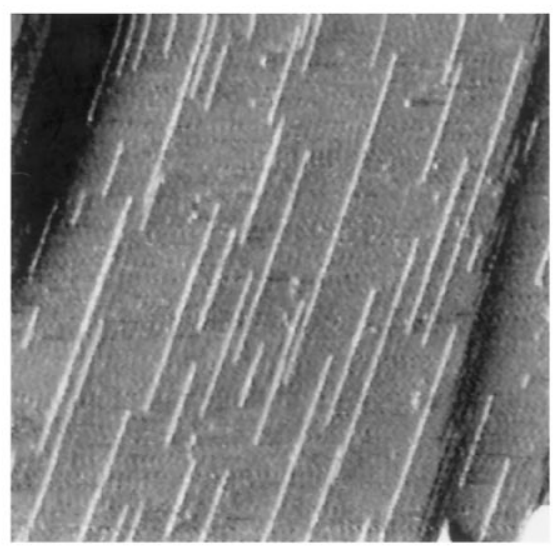

d) $\mathrm{T}=350 \mathrm{~K}$

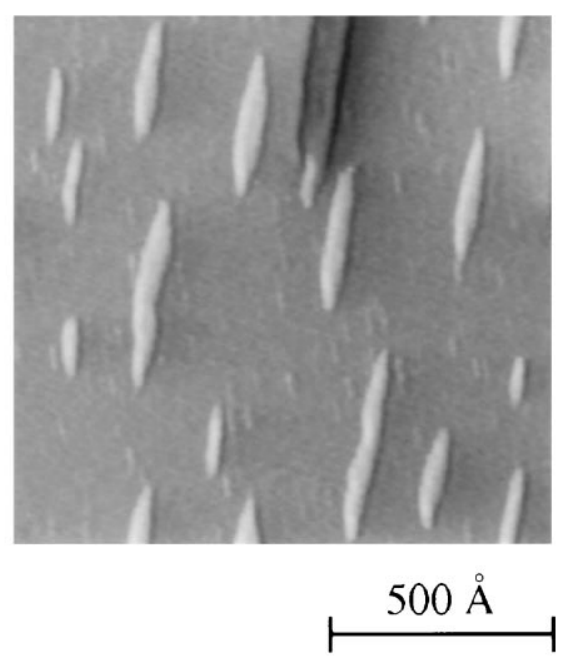

Fig. 17. STM images of elongated $\mathrm{Cu}$ islands on $\mathrm{Pd}(110)$ grown and imaged at the indicated temperatures for $0.1 \mathrm{ML}$ (a), (c), and (d), and 0.07 ML (b) coverage, respectively (from Ref. [206]). 
experiments revealed in addition that the system is quite complex as the attaching species are entities comprised of four $\mathrm{Si}$ atoms. ${ }^{33}$

The first example of 1D islands on metal surfaces was reported for the nucleation of $\mathrm{Cu}$ on $\operatorname{Pd}\left(\begin{array}{lll}1 & 1 & 0\end{array}\right)$ [22,206]. The STM images reproduced in Fig. 17 show a transition from 1D to 2D islands occurring with increasing deposition temperature. The islands formed below room temperature are monoatomic chains of $\mathrm{Cu}$ atoms. This conclusion comes from their imaging width and the fact that lateral island separations down to two lattice constants along $\langle 001\rangle$ could be discerned [206]. The formation of $\mathrm{Cu}$ wires up to $1000 \AA$ long was reported, corresponding to aspect ratios as large as $A \approx 300$ [22]. The islands become compact above $300 \mathrm{~K}$, but are still elongated along $\langle 1 \overline{1} 0\rangle$.

The quantitative analysis for this system was performed using Arrhenius plots of 2D and 1D island densities measured at $\Theta=0.1 \mathrm{ML}$. From Fig. 18(a) it is inferred that the 1D density measured across the troughs stays constant for temperatures below $260 \mathrm{~K}$, whereas the $2 \mathrm{D}$ density shows an Arrhenius slope. This was interpreted as indicative of true 1D diffusion. Accordingly, 1D nucleation theory
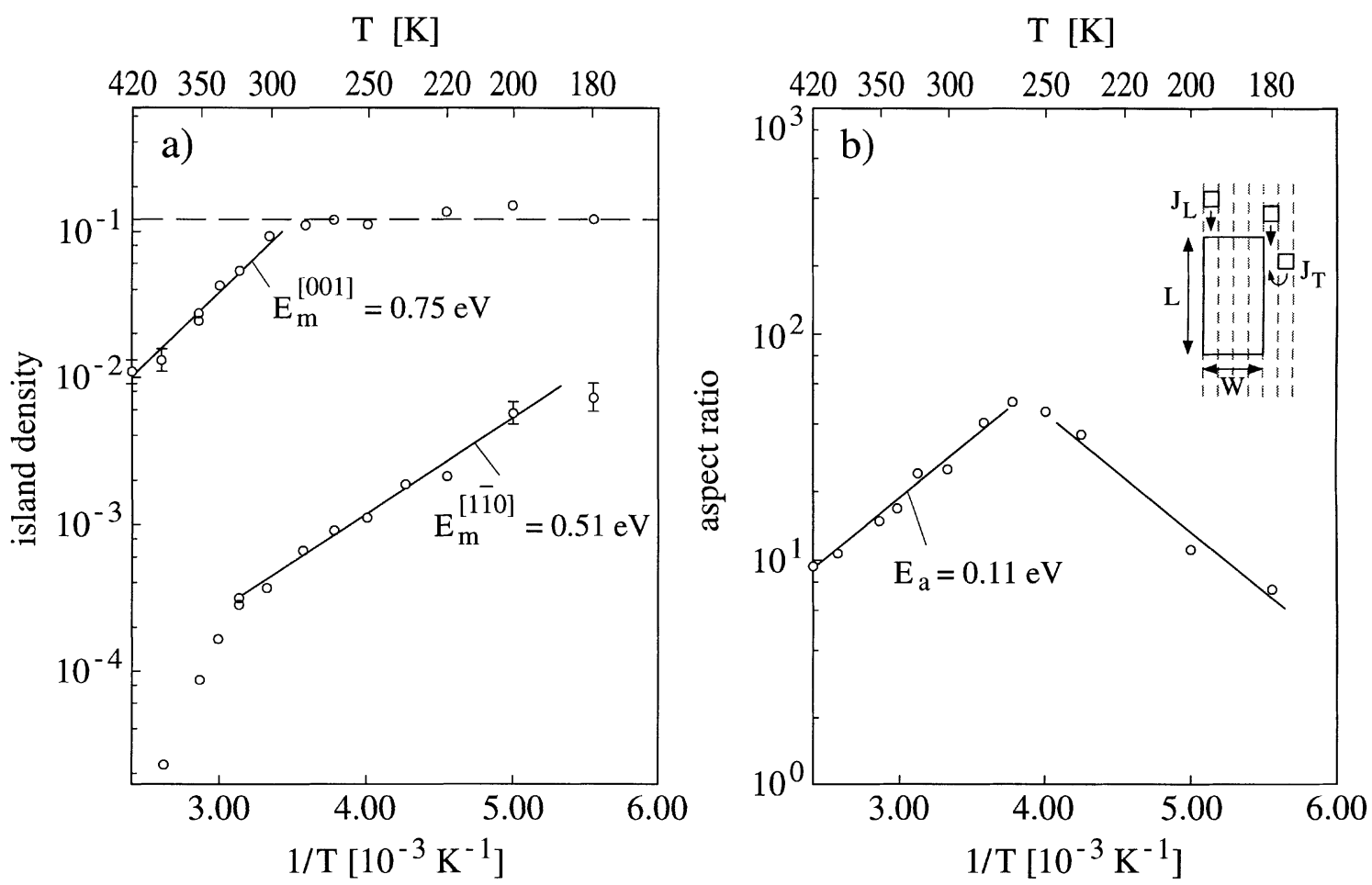

Fig. 18. Quantitative analysis for $\mathrm{Cu} / \mathrm{Pd}\left(\begin{array}{lll}1 & 1 & 0\end{array}\right)$. (a) Arrhenius plot of the $1 \mathrm{D}$ island density across the troughs (upper curve) and the overall 2D density (lower curve). (b) Arrhenius plot of the aspect ratio $A=L / W, L$ and $W$ being the length and width of islands. The inset shows a model of an island with the diffusion channels being indicated by broken lines; $J_{\mathrm{L}}$ and $J_{\mathrm{T}}$ denote the longitudinal and transversal diffusion rates, respectively (from Ref. [206]).

\footnotetext{
${ }^{33}$ An alternative explanation for elongated islands proposed an exchange mechanism [205]. This model could be ruled out by the experimental finding of constant growth rates of islands as a function of their length [204] in contradiction to the prediction by the authors of Ref. [205].
} 
$[57,67,70,207]$ was applied to the slope of the $2 \mathrm{D}$ density to yield an activation energy of $E_{\mathrm{m}, \mathrm{L}}=$ $0.51 \pm 0.05 \mathrm{eV}$ for longitudinal displacement along the troughs. ${ }^{34}$ The $1 \mathrm{D}$ island density has an absolute value of 0.1 islands per lattice site which precisely equals the coverage. The $\mathrm{Cu}$ wires formed on $\operatorname{Pd}\left(\begin{array}{lll}1 & 1 & 0\end{array}\right)$ below room temperature are thus determined to be one atom wide.

The linear decrease of the $1 \mathrm{D}$ density above $265 \mathrm{~K}$ was interpreted as the onset of diffusion across the troughs. The opening up of an additional diffusion channel at that temperature also affects the $2 \mathrm{D}$ density. A steeper slope is seen only above $320 \mathrm{~K}$, however, as will be discussed below. Since diffusion takes place in two directions, albeit with different rates, the question arises as to whether the system can still be treated within 1D nucleation theory. It was reasonable to assume that the decrease of the 1D density solely reflects the activation energy for cross-channel diffusion as it constitutes the rate limiting step for $T>300 \mathrm{~K}$. The authors thus treated the problem as a $1 \mathrm{D}$ case and arrived at $E_{\mathrm{m}, \mathrm{T}}=$ $0.75 \pm 0.07 \mathrm{eV}$ for diffusion across the troughs [206], assuming $i=1$ also for that regime.

The aspect ratio $A$ bears complementary information corroborating the above interpretation. Below $265 \mathrm{~K}$, it simply reflects the increase in monoatomic chain length due to increasing diffusion along the troughs. Above this temperature, cross-channel diffusion leads to an increased width $W$. As the activation energy for cross-channel diffusion is larger than that for in-channel diffusion, the change in $W$ with temperature is more rapid than the increase in length $L$. Accordingly, the slope of $A$ for $T>265 \mathrm{~K}$ was interpreted as reflecting the difference between the two diffusion barriers. This yielded a value of $0.22 \mathrm{eV}$, in full agreement with the results obtained from treating island densities within the framework of 1D nucleation.

The diffusion mechanism suggested for cross-channel diffusion by the EAM calculations included in this study is the exchange [206]. This diffusion mechanism has often been observed in FIM studies on fcc(110) surfaces [208-212]. Although one would expect exchange in heteroepitaxy to lead to alloying, as seen for $\mathrm{Au} / \mathrm{Ni}\left(\begin{array}{lll}1 & 1 & 0\end{array}\right)$ [213], $\mathrm{Ni} / \mathrm{Au}\left(\begin{array}{lll}1 & 1 & 0\end{array}\right)$ [214], and $\mathrm{Au} / \mathrm{Ag}\left(\begin{array}{lll}1 & 1 & 0\end{array}\right)$ [215], this is not necessarily always the case. Substrate atoms that have been ejected can immediately take part in the next exchange when encountering a substitutional atom of the adspecies thus bringing it back into the surface again. It depends on the relative activation energies whether exchange in one or the other direction is faster, signifying that some systems stay alloyed after exchange and others do not. Interpreting the EAM results in terms of a Pd atom promoting the second exchange step, the total barrier for a side walk of a $\mathrm{Cu}$ atom is $0.25 \mathrm{eV}$ larger than that for walking along the troughs, in agreement with the experimental analysis.

Recently, the $\mathrm{Cu} / \mathrm{Pd}\left(\begin{array}{lll}1 & 1 & 0\end{array}\right)$ system was returned to by $\mathrm{Li}$ et al. [216]. These authors performed KMC simulations that showed nucleation on fcc(1 10$)$ surfaces in a new light. Li et al. generally assume that diffusion within a trough adjacent to an island is not affected by the island, whereas atoms at the ends of 1D strings or 2D islands are immobilized (see Fig. 19(a)). These assumptions reflect the weak crosschannel interactions and strong binding within the channels generally expected from geometric arguments for fcc( 110$)$ surfaces. In their first model, Li et al. show that the barriers formerly extracted are too large (see the shift of theoretical with respect to experimental aspect ratios in panel I of Fig. 19(b); the simulated islands, although 1D, are much too short at low temperature). In a second

\footnotetext{
${ }^{34}$ This analysis implies knowledge of the critical cluster size. On fcc(1 10$)$ surfaces this information can readily be obtained from the onset of Ostwald ripening for monoatomic chains. As atoms at the ends of these chains are only singly bonded, this situation corresponds to the onset of Ostwald ripening for dimers on close-packed surfaces described in Section 3.2. From the absence of coarsening in successive images of the monoatomic chains, $i=1$ holds for the present system at $T<300 \mathrm{~K}$.
} 
a)

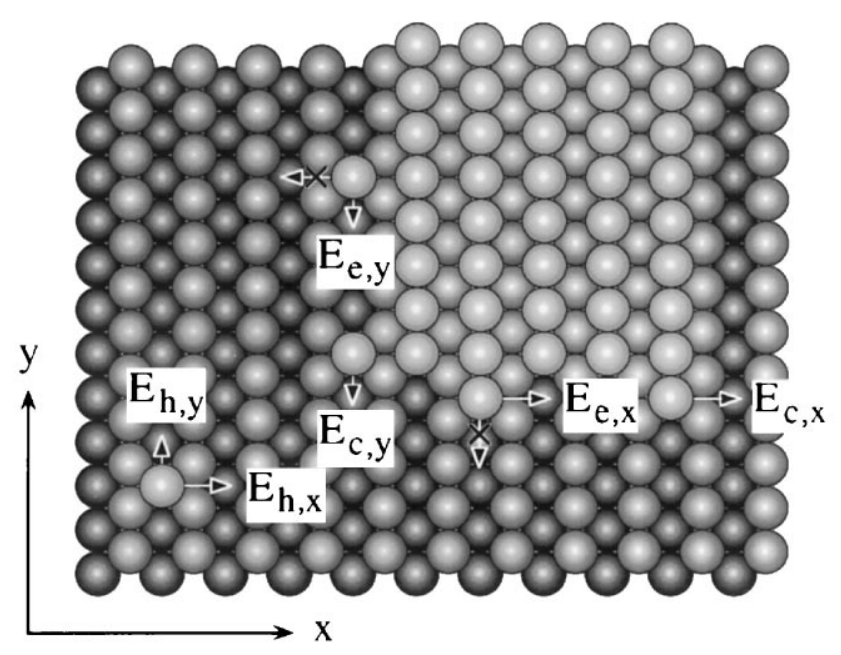

\begin{tabular}{l|cccccc}
\hline & $\mathrm{E}_{\mathrm{h}, \mathrm{x}}$ & $\mathrm{E}_{\mathrm{h}, \mathrm{y}}$ & $\mathrm{E}_{\mathrm{e}, \mathrm{x}}$ & $\mathrm{E}_{\mathrm{e}, \mathrm{y}}$ & $\mathrm{E}_{\mathrm{c}, \mathrm{x}}$ & $\mathrm{E}_{\mathrm{c}, \mathrm{y}}$ \\
\hline Model I & 0.75 & 0.51 & 1.00 & 0.51 & 1.00 & 0.51 \\
Model II & 0.40 & 0.40 & 0.75 & 0.40 & 0.75 & 0.40 \\
Model III & 0.45 & 0.30 & 0.65 & 0.30 & 0.65 & 0.30 \\
\hline
\end{tabular}
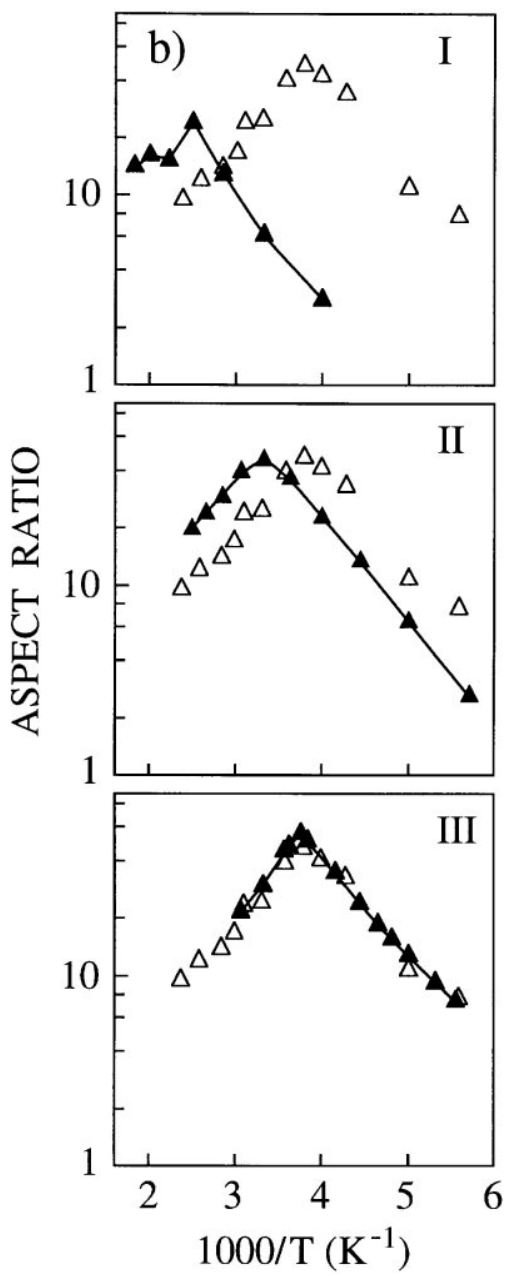

Fig. 19. Anisotropic corner rounding as the dominant mechanism giving rise to elongated islands on fcc(1 1 0 ). (a) Ball model showing the processes considered. Their barriers are tabulated. (b) Simulated aspect ratios (filled symbols) from three parameter sets I, II, and III in comparison with experimental results (open symbols) (from Ref. [216]).

approach, $\mathrm{Li}$ et al. show that $1 \mathrm{D}$ islands are also obtained for isotropic 2D diffusion, as long as there is a difference in attachment at both island sides. In the simulations this was established by immobilizing atoms arriving at island ends (high values for $E_{\mathrm{e}, x}$ and $E_{\mathrm{c}, x}$ ), while atoms in troughs adjacent to an island were allowed to diffuse along the side and finally leave the island that way. This corresponds to zero effective sticking at the sides, whereas sticking is unity at the ends. As there is no cross-channel interaction, the only way an island can become wide is nucleation of a second island in an adjacent trough. This is made improbable since the atoms that have left an island via edge diffusion can then perform a cross-channel jump into the trough occupied by the island and finally end up at the short island edge. This results in a net flux from long to short edges and leads to the production of monoatomic chains. Since this movement can be considered as diffusion around corners, although involving temporal detachment from the islands to the terrace, the process was labeled "anisotropic 
corner-rounding". In contrast to this isotropic diffusion model, however, new experiments investigating the flux dependency, determined diffusion to be anisotropic at $280 \mathrm{~K}$ [216]. Also the agreement of the isotropic diffusion model with experimental aspect ratios is still not sufficient (see panel II, Fig. 19(b)). In particular, the 1D island density has no real constant regime (not shown here), a necessary consequence of cross-channel diffusion being activated simultaneously with in-channel diffusion.

Therefore, a third and final proposal was made: a model incorporating three energy barriers as parameters. Going back to the original interpretation, diffusion is anisotropic. The new ingredient is that barriers are lower than formerly assumed and therefore cross-channel diffusion becomes activated much earlier opening up a net flux from island sides to their ends. This model yields excellent agreement with experiment for both the Arrhenius plot of island densities (not shown here) and aspect ratios of the island dimensions (panel III, Fig. 19(b)). It also describes the experimental flux dependency.

The essential point learned from the comparison to KMC simulations is that anisotropic diffusion and anisotropic sticking are both essential for an adequate description of nucleation on fcc(llll 10$)$ surfaces. Anisotropic sticking is more important for the island shape and anisotropic diffusion

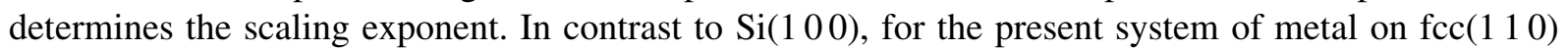
metals sticking to islands is higher along the fast direction of diffusion and therefore sticking and diffusion anisotropy amplify each other. Due to this amplification, the uni-dimensional island regime is extended to temperatures far beyond the strictly $1 \mathrm{D}$ diffusion regime. For $\mathrm{Cu} / \mathrm{Pd}$, it emerges that the pure $1 \mathrm{D}$ regime ends well below the critical temperature $T_{\mathrm{c}}=265 \mathrm{~K}$, defined as where the aspect ratio has its maximum. Up to $T_{\mathrm{c}}$ monoatomic chains form. The value of this critical temperature essentially depends on the ratio of sticking and edge diffusion at the island sides to that at island ends, and not on the degree of diffusion anisotropy [216]. The slope of the total island density in the regime below $T_{\mathrm{c}}$ therefore is not directly related to the barrier for fast diffusion.

The irreversible sticking to island ends is lifted above $T_{\mathrm{c}}$ through edge diffusion along, and away from the short island edges. ${ }^{35}$ This increases the island width and decreases the $1 \mathrm{D}$ density. However, the overall density keeps its slope. A third regime is entered at the point where the overall island density changes slope $(325 \mathrm{~K}$ for $\mathrm{Cu} / \mathrm{Pd}(110))$. It is likely that this last transition is caused by a change in critical nucleus, i.e. by dimers becoming unstable through bond breaking within the troughs. The slope of the $1 \mathrm{D}$ island density is related to the barrier for cross-channel diffusion only in a non-trivial manner. Therefore the $\mathrm{Cu} / \mathrm{Pd}\left(\begin{array}{lll}1 & 1 & 0\end{array}\right)$ system escapes from a straightforward analysis where slopes are directly related to diffusion barriers. Consequently, absolute values for the barriers were incorrect in the former interpretation. The basic feature of anisotropic diffusion being responsible for 1D islands is still valid, however, though in a temperature range much smaller than formerly believed.

The physical picture evolving for nucleation and growth on fcc(110) surfaces is that anisotropic diffusion goes hand in hand with anisotropic sticking giving rise to elongated island shapes over a wide range of temperatures. A model incorporating diffusion and sticking consistently describes the results for $\mathrm{Cu} / \mathrm{Pd}$. Recently, experimental results for $\mathrm{Pd} / \mathrm{Pd}\left(\begin{array}{lll}1 & 1 & 0)\end{array}\right.$ [217,218] and $\mathrm{Pt} / \mathrm{Pt}\left(\begin{array}{lll}1 & 1 & 0\end{array}\right)$ [41] have become available, opening up a more general account for nucleation on fcc(1 10$)$ surfaces in the near future.

\footnotetext{
${ }^{35}$ Although edge diffusion along the short ends opens up a way of escaping from an island, it is apparently not a change in critical nucleus size as island densities in experiment and simulation still scale as for $i=1$. This is an example where one reaches the limits of the definition of critical nucleus. Strictly speaking, a dimer is unstable as it often dissociates via edge diffusion on the timescale of deposition. However, dissociation does not occur the same way as predominant formation of stable nuclei, namely within troughs, therefore irreversible dimer formation along the troughs still guarantees $i=1$ scaling.
} 


\subsubsection{Diffusion anisotropy on fcc(100)-hex}

The (1 000$)$ surfaces of $\mathrm{Au}, \mathrm{Pt}$, and Ir reveal the so-called hex-reconstruction [219]. The structure of this surface reconstruction has been characterized by a wide range of experimental techniques, e.g. for $\mathrm{Au}$, with LEED [220], RHEED [221], helium atom scattering (HAS) [222], STM [223], and TEM [224], as well as X-ray diffraction [225-228]. It is characterized by a contracted quasi-hexagonal (hex) layer resting on top of the square substrate. The orientation of this layer is such that one of the three close-packed directions in the hexagonal overlayer is aligned to one of the two close-packed rows of the substrate. ${ }^{36}$ This leads to two rotational domains [219,225]. The incommensurations caused by the stacking of a layer with hexagonal symmetry on a square lattice are nicely seen as different imaging heights in the STM image of $\mathrm{Pt}\left(\begin{array}{l}10 \\ 0\end{array}\right)$ reproduced in Fig. 20(a). Similar to moiré patterns these are long range undulations in the adsorption height of the surface layer. However, there is a marked anisotropy between the two $\langle 1 \overline{1} 0\rangle$ directions. Perpendicular to the direction of alignment (which is indicated with a white bar, Fig. 20(a)), the close-packed rows show strong height modulations of $0.5 \AA$ [222,227], caused by changes in stacking from on-top to hollow (six atomic rows reside on five rows of the square substrate, see ball model in Fig. 20(a)). The modulation is much weaker parallel to the direction of alignment. Along $\langle 1 \overline{1} 0\rangle$, on the average, 21 atoms sit on 20 bulk sites inducing less dramatic site changes. More precisely, within one atomic row the atoms either change between hollow and bridge or between top and bridge sites, but never from hollow to top. The corrugation along the atomic rows was estimated from HAS to be below $0.1 \AA$ [222].

\section{a)}

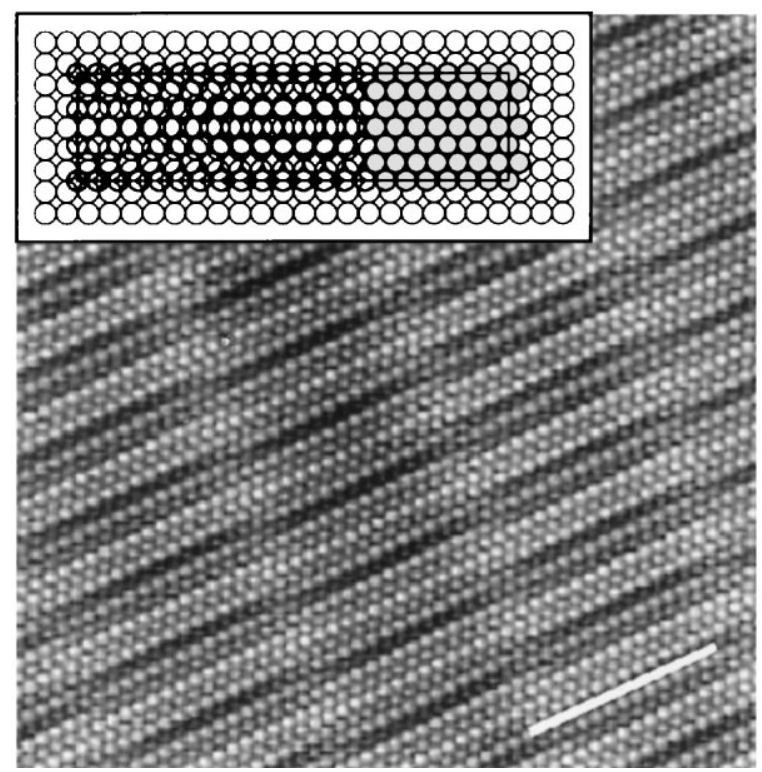

b)

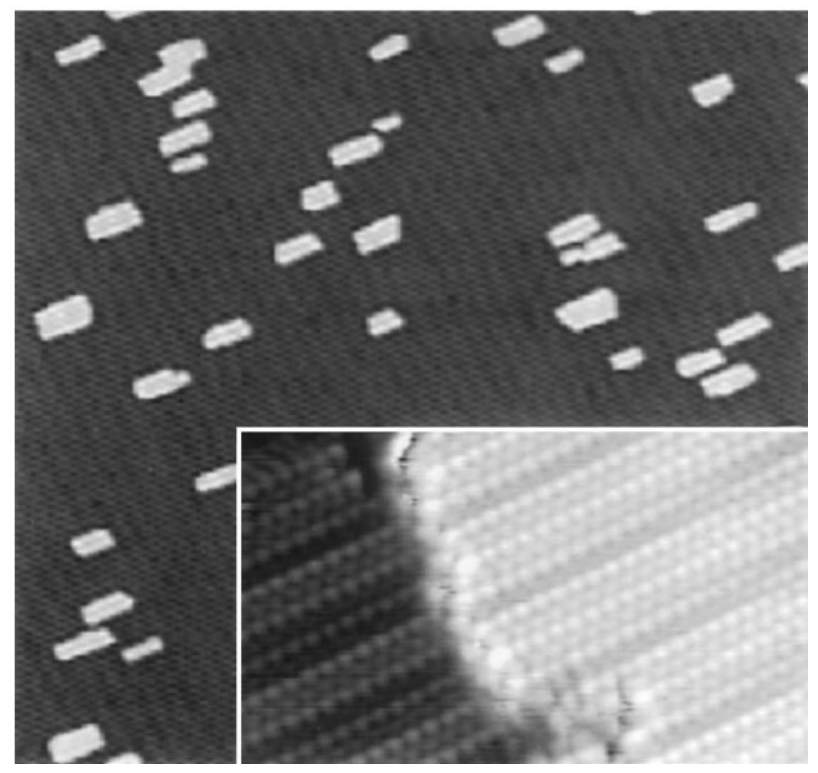

Fig. 20. STM images of (a) the $\operatorname{Pt}(100)$-hex surface $\left(140 \times 140 \AA^{2}\right)$, (b) monoatomically high islands obtained after Pt deposition $\left(F=2.3 \times 10^{-4} \mathrm{ML} / \mathrm{s}, T=389 \mathrm{~K}, 1400 \times 1400 \AA^{2}\right)$. Inset in (a): Ball model for the $(5 \times 20)$-unit cell. Inset in (b): Edge of island, atomically resolved showing the reconstruction on top of the islands (from Ref. [83]).

\footnotetext{
${ }^{36}$ Domains with a slight rotation are also observed [229].
} 
The anisotropy in surface corrugation reflects itself in diffusion anisotropy on this substrate. Tentatively, one would expect diffusion to be faster along those close-packed rows which are aligned to the substrate as the surface is smoother in that direction. Homoepitaxy on $\operatorname{Au}(100)$ and $\operatorname{Pt}(100)$ yields elongated islands (see Fig. 20(b) and Refs. [83,102]). The islands are reconstructed, implying that the substrate reconstruction is locally lifted in the course of island growth. In both cases, there were clear indications of strong diffusion anisotropy. For $\mathrm{Au}(100)$, zones denuded of islands were found at steps running perpendicular to the aligned rows, whereas for steps parallel to these rows this effect was absent $^{37}$ [102]. This identifies fast diffusion to be along the aligned close-packed rows marked by the white bar in Fig. 20(a). The same depletion zones are present between islands. Diffusion anisotropy

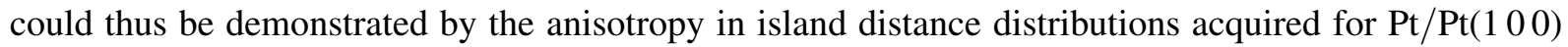
[83]. With respect to fcc(110) surfaces nucleation on the hex-reconstructed fcc(100) surfaces is characterized by isotropic sticking to islands since they are forming on a hexagonal layer. Consequently, much smaller aspect ratios, ranging from 3 to 8 depending on the deposition conditions, have been reported [102].

The interpretation of island densities was based on slightly different models for the two systems. Günther et al. [102] found for $\mathrm{Au} / \mathrm{Au}(100)$ a flux dependency at $315 \mathrm{~K}$ with an exponent of $\chi=0.37 \pm$ 0.03. From simulations incorporating reasonable assumptions on the order of magnitude of the attempt frequency and sticking to the islands with unity, isotropic diffusion with $i=1$ could be ruled out. The exponent was interpreted as evidence for strongly anisotropic diffusion (in agreement with the depletion zones) and reversible growth with $i=3$. The Arrhenius data $(315-380 \mathrm{~K})$ were interpreted in terms of mean-field theory for infinitely anisotropic diffusion yielding $E_{\mathrm{m}}=0.2 \mathrm{eV}, E_{3}=0.6 \mathrm{eV}$. A model with finite diffusion anisotropy $(1: 100-1000)$ does not affect these values very much [102].

For Pt/Pt(1 00 ) Linderoth et al. [83] started from a statistical analysis. By comparing experimental autocorrelation functions for island positions to those produced by a KMC simulation, they inferred the diffusion anisotropy to be $1: 1000$ (with fast diffusion along the aligned rows, similar to Au(10 0)). The authors further showed that experimental island size distributions also scale into common curves for the anisotropic case. Comparison with size distributions created in KMC models excluded dimer mobility and provided additional support for diffusion anisotropy. The experimental island densities obtained between 318 and $497 \mathrm{~K}$ at various deposition fluxes $F$, all fell onto a common line when plotted as $\log \left(n_{x}\right)$ vs. $\left(F / \nu_{0}\right) \exp (-E / k T)$. This allows the data to be described with a single critical nucleus size and an exponent of $0.27 \pm 0.01$, rather close to $\frac{1}{4}$ expected for $1 \mathrm{D}$ diffusion with $i=1$. An effective barrier in the direction of fast diffusion of $E_{\mathrm{m}}=0.43 \pm 0.03 \mathrm{eV}$ was obtained under the assumption of $\nu_{0}=10^{13} \mathrm{~s}^{-1}$.

Both studies agree on the importance of diffusion anisotropy and on sticking to islands being isotropic. Their experimental data are consequently analyzed within quite similar models. However, the activation energies for $\mathrm{Au}$ are considerably smaller than for $\mathrm{Pt}$, involving dimer instability for the former. ${ }^{38}$ Dimers are stable and immobile up to $500 \mathrm{~K}$ for $\mathrm{Pt}(100)$. This chemical difference is in line with theory. Calculations report barriers for self-diffusion on the hexagonally close-packed (1 111$)$

\footnotetext{
${ }^{37}$ The same effect was found for A- and B-steps on $\mathrm{Si}(100)$ [202]. Anisotropic diffusion also manifests itself in early coalescence. For high diffusion anisotropies, the extent of an island in the direction of slow diffusion can become comparable to the mean free diffusion path in that direction at small coverages. This gives rise to an onset of coalescence $\left(\Theta_{\text {sat }} \approx 0.07 \mathrm{ML}\right)$ earlier than in the case of isotropic diffusion [83].

${ }^{38}$ Bartelt et al. [81] also claim significant dimer mobility for $\mathrm{Au} / \mathrm{Au}(100)$.
} 
surfaces to be smaller for $\mathrm{Au}(0.15 \mathrm{eV}$ [159], $0.22 \mathrm{eV}$ [179]) than for Pt (0.38 eV [126], $0.39 \mathrm{eV} \mathrm{[159]).}$ Of the low index surfaces this surface orientation resembles closest the morphology of the hexreconstructed (100) face, and these theoretical values agree well with the experimental ones for diffusion on the quasi-hexagonal reconstructed (100) surfaces of Au and Pt.

Despite the consistent image of nucleation on anisotropic substrates derived so far, it should be noted that the precise value of the scaling exponent for anisotropic surfaces is still a matter of debate. Whereas point island models for anisotropic diffusion consistently find $\chi=\frac{1}{4}[57,67,83,207,230],{ }^{39}$ KMC simulations accounting for the finite island size find a value closer to $\frac{1}{3}$ (exactly $\frac{1}{3}$ in Ref. [231], $0.30 \pm 0.01$ for infinite anisotropy, and 0.32 for finite anisotropy of $1: 1000$ in Ref. [83]). In these KMC models sticking is isotropic. Anisotropic sticking leads back to the result from point island models, for example the simulations by Li et al. [216] yield $\frac{1}{4}$ for anisotropic and $\frac{1}{3}$ for isotropic diffusion. The reason for which simulations assuming anisotropic sticking show the exponent for point islands is that islands are quite narrow perpendicular to the direction of fast diffusion, making them point targets. The open question therefore is whether systems with anisotropic diffusion and isotropic sticking scale with the point island value of $\frac{1}{4}$, or the one of finite islands which lies closer to $\frac{1}{3}$. The experimental value of 0.27 from Linderoth et al. seems to lie closer to the point island value although the islands have finite size. This is presumably due to a reduction of effective island size, as blocking effects become smaller if fast diffusion is restricted to one channel every six atomic rows [83].

The examples discussed in this section demonstrate that the analysis of nucleation and growth and the determination of migration barriers is much less straightforward on anisotropic lattices than for isotropic surfaces. Terrace diffusion, edge diffusion, and sticking to islands may all be anisotropic. These effects are a priori difficult to discern. However, the examples set so far show that the task can be accomplished with detailed experimental information carefully analyzed, mainly within the framework of KMC models. Sticking anisotropy is a general characteristic of fcc(1 110$)$ surfaces. It acts in the same direction as possible diffusion anisotropy, although this is not necessarily needed to account for elongated islands. On the other hand, strong diffusion anisotropy characterizes hex-reconstructed fcc(1 00$)$ surfaces, whereas sticking to islands is isotropic. On both surfaces elongated islands form. As the island shape is mainly determined by sticking, fcc( $(110)$ surfaces are ideally suited to grow islands which are elongated down to the monoatomic chain limit. These structures represent model systems allowing the investigation of physical properties as magnetism, conductivity and superconductivity in one dimension.

\subsection{Nucleation and self-organization on patterned substrates}

The strain energy present in heteroepitaxial systems can lead to a transition from pseudomorphic to weakly incommensurate layers. Together with certain surface reconstructions, these layers obey dislocations which generally interact repulsively. This repulsion is of extremely long range, which can be understood as mediated by the strain field. This long range interaction has two general implications. Firstly there is a well-defined density of dislocations due to the compromise between optimum strain relief achieved through introduction of dislocations and their mutual repulsion. Secondly the dislocations order into regular patterns. Examples are the herringbone reconstruction of $\mathrm{Au}(111)$, or

\footnotetext{
${ }^{39}$ The slightly different value of $\frac{2}{7}$ formerly proposed by Mo et al. [76] was corrected in a comment by Pimpinelli et al. [230] to $\frac{1}{4}$, Mo et al. [231] agreed in that point.
} 
dislocation networks where crossing of dislocations is realized (for a general account of strain relief on fcc(1 111 1) surfaces see Ref. [29]).

There exist indications and clear quantitative evidence (see below) that dislocations can represent repulsive barriers for diffusing adatoms. Dislocations can therefore strongly influence nucleation island densities $[150,151,189]$. Their influence on nucleation can reach as far as driving the most perfect layerby-layer growth observed so far. ${ }^{40}$

These two ingredients, ordering of dislocations into periodic patterns and strong influence of dislocations on adatom diffusion, can be employed to grow periodic arrays of almost monodispersed islands [150,235]. In this section we will discuss results obtained for nucleation on such patterned substrates. Our first example will be the one where ordering was long believed to be caused by the influence of dislocations on diffusion, namely, $\mathrm{Ni}$ nucleation at the "elbows" of the Au(111) reconstruction. Recent STM observations have revealed, however, that site selective exchange gives rise to nucleation at elbows. After this we will address two systems with pure adatom diffusion: The nucleation of $\mathrm{Al}$ on $\mathrm{Au}\left(\begin{array}{lll}1 & 1 & 1\end{array}\right)$ and that of $\mathrm{Ag}$ on a regular dislocation network formed by the second $\mathrm{Ag}$ layer on $\mathrm{Pt}(111)$. Both examples show considerable deviations from the classical scaling laws describing homogeneous substrates (either isotropic or anisotropic ones). The substrates addressed below are inhomogeneous, i.e. diffusion barriers and adsorption energies depend on where the particle is located within the mesoscopic structure. At the end of this section we will show further examples for inhomogeneous substrates where periodic island arrays may be grown through self-organization.

\subsubsection{Site selective nucleation through exchange on Au(l 111$)$}

$\mathrm{The} \mathrm{Au}\left(\begin{array}{lll}1 & 1 & 1\end{array}\right)$ herringbone reconstruction is driven by the considerable tensile stress in the outermost layer [236-238]. Dense domain walls, or partial dislocations, separate alternating fcc and hcp stacking regions. A $4 \%$ compression of the surface layer is achieved by two partial dislocations per $(\sqrt{3} \times 22)$ unit cell. One extra atom is inserted giving 23 surface atoms on top of 22 second-layer atoms along the close-packed $\langle 1 \overline{1} 0\rangle$-directions [238,239]. Energy considerations bear that more fcc than hcp sites are populated, giving rise to a pairwise arrangement of the $\langle 11 \overline{2}\rangle$-oriented domain walls. Locally, the compression is unidirectional, on larger terraces a mesoscopic order of the domain walls is established. In order to reduce the anisotropy of the surface stress tensor [240] the domain walls bend by $\pm 120^{\circ}$ with a period of $250 \AA$ (see Fig. 21(a)). This pattern of dislocations forms the so-called herringbone structure.

Chambliss and coworkers [241-243] showed that the elbows of this reconstruction act as sites of preferred nucleation for $\mathrm{Ni}$ deposited at room temperature. This leads to quite regularly spaced islands accompanied by a narrow island size distribution (see Figs. 21(b) and (c)). Already at that time, the advantage of this self-ordering for possible applications was pointed out.

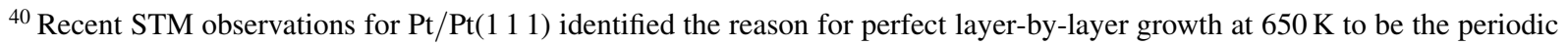
appearance of dislocations on the topmost growth layer caused by island coalescence [232]. Above $400 \mathrm{~K}$ the $\operatorname{Pt}(1111)$ surface is known to reconstruct in the presence of Pt adatoms [233]. The dislocations associated with the reconstruction strongly reduce the mobility on the substrate terraces leading to a high island density. The islands themselves do not reconstruct, presumably they are too small. Therefore the mobility on their surface remains high giving adatoms many opportunities to descend. The interlayer barrier can thus readily be overcome and islands grow 2D. When they coalesce into large patches, the reconstruction floats up and diffusion is again slowed down. This causes perfect layer-by-layer growth. More than 150 almost undamped oscillations have been observed in reflected He intensity, where a much faster decay of the amplitude was expected without the periodic appearance of the reconstruction [232,234].
} 

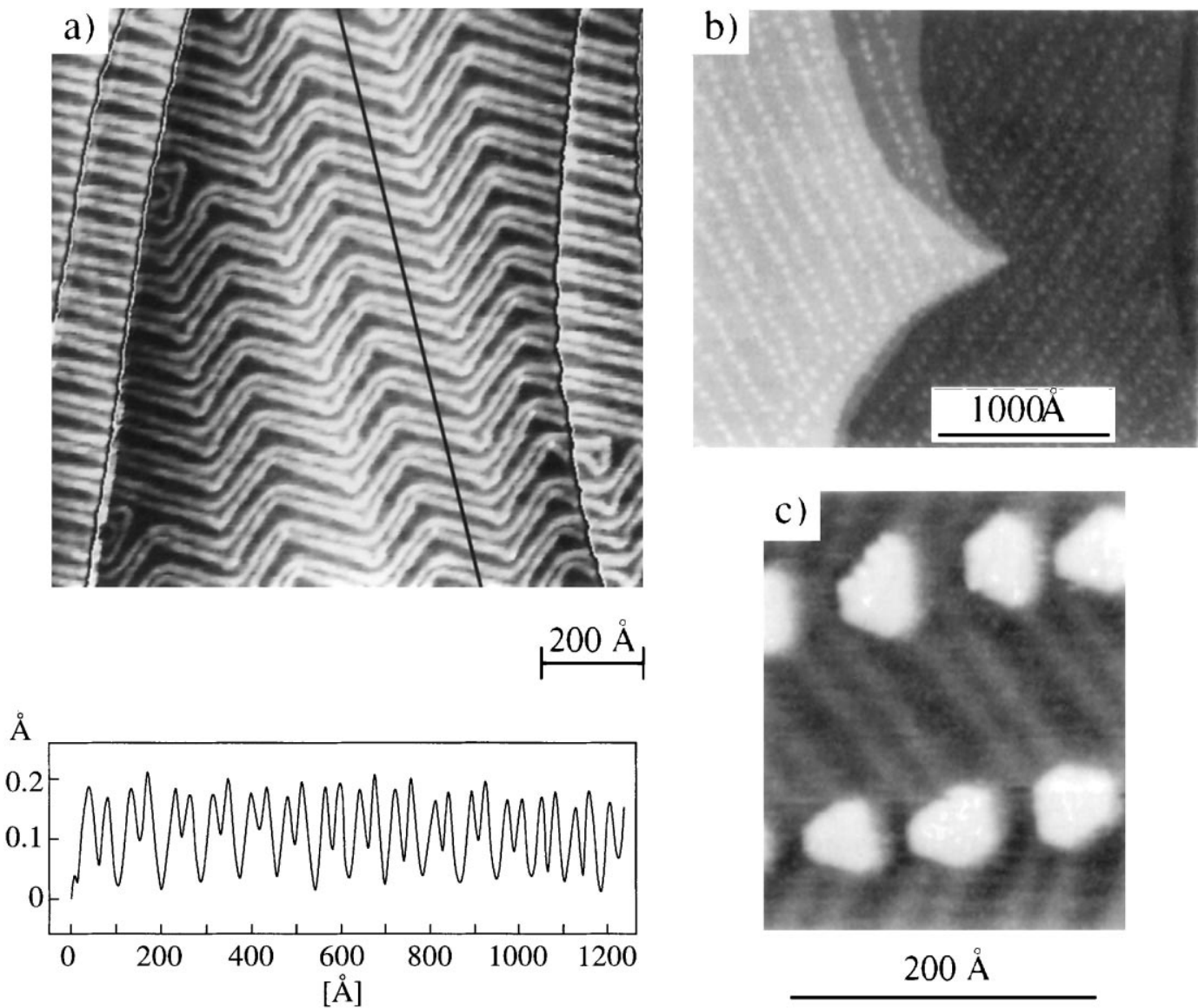

Fig. 21. (a) STM image of the herringbone pattern characterizing the mesoscopic order of the $\operatorname{Au}\left(\begin{array}{lll}1 & 1 & 1\end{array}\right)(\sqrt{3} \times 22)-$ reconstruction. Partial dislocations appear bright since there atoms populate bridge sites (from Ref. [238]). (b) and (c) Ni nucleation on that surface at room temperature. (b) Large scale STM image showing monolayer Ni islands aligned in rows along the $\langle 11 \overline{2}\rangle$-directions $(\Theta=0.11 \mathrm{ML})$. (c) Detail showing that these Ni islands are located at elbows of the herringbone reconstruction $(\Theta=0.14 \mathrm{ML})$ (from Ref. [241]).

The distinctive nucleation probability at elbows of the reconstruction was ascribed by Chambliss et al. [24] to attractive potential wells which trap diffusing adatoms. It has been shown in a Monte-Carlo simulation that even small trapping probabilities at the elbows may be sufficient to cause the experimentally observed alignment of islands [241]. In a recent re-examination of the $\mathrm{Ni} / \mathrm{Au}\left(\begin{array}{lll}1 & 1 & 1\end{array}\right)$ system the origin of the potential wells was uncovered [244]. It was found to be due to site selective place exchange of $\mathrm{Ni}$ adatoms with Au surface atoms localized at the elbows. This is followed by preferential nucleation of $\mathrm{Ni}$ adislands on top of substitutional Ni islands. The STM images represented in Fig. 22 demonstrate this effect. The reason for preferred place exchange at elbows is thought to be the reduced coordination in these highly strained areas. At the elbows a close-packed atomic row terminates as STM images of these regions directly demonstrate [229,241]. As a result, there are Au atoms with reduced lateral coordination. These atoms are more susceptible to place exchange than those residing on the terraces. 
a)

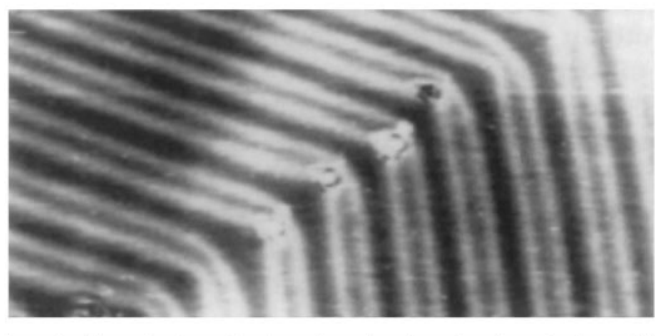

Element Surface free energy $(\mathrm{eV})$ Heat of sublimation $(\mathrm{eV})$

\begin{tabular}{lll}
\hline $\mathrm{Ag}$ & 0.50 & 2.95 \\
$\mathrm{Al}$ & 0.56 & 3.39 \\
$\mathrm{Cu}$ & 0.69 & 3.51 \\
$\mathrm{Au}$ & 0.72 & 3.79 \\
$\mathrm{Ni}$ & 0.90 & 4.45 \\
$\mathrm{Co}$ & 0.94 & 4.40 \\
$\mathrm{Fe}$ & 0.96 & 4.32 \\
\hline
\end{tabular}

b)

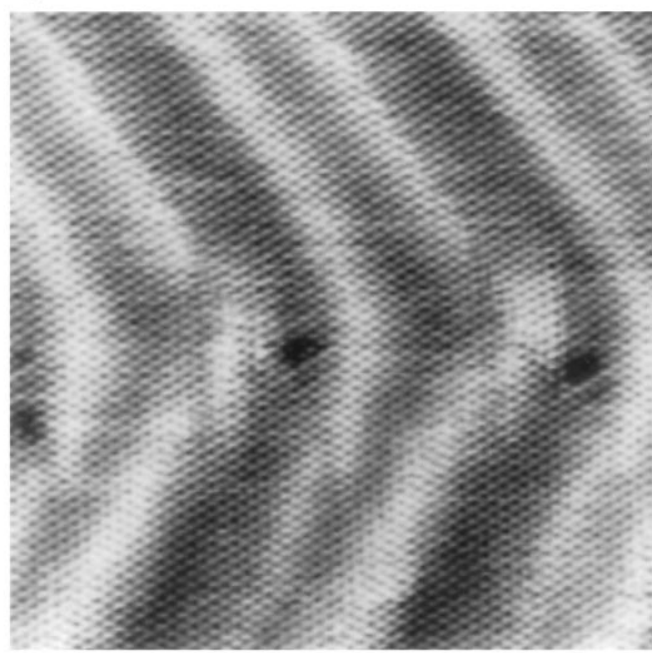

Fig. 22. Preferential nucleation at elbows due to site selective exchange. (a) Some of the elbows that are not yet covered by islands reveal depressions $(\Theta=0.002 \mathrm{ML}$, and $T=300 \mathrm{~K}$ ). (b) The formation of adislands can be suppressed by performing adsorption at $350 \mathrm{~K}$. This gives access to the depressions in more detail revealing that they consist of substitutional Ni islands comprised of $4-5$ atoms each $(\Theta=0.002 \mathrm{ML})$. The table shows the energies that permit estimation of which metals could perform place exchange with Au (see text) (from Ref. [244]).

Preference of nucleation at or close to elbows of the Au reconstruction was also observed for room temperature deposition of several more metals than Ni, for example Fe [245,246], Co [247] and $\mathrm{Rh}$ [248]. For other metals, on the other hand, nucleation was found to be homogeneous and independent of the mesoscopic order of the $(\sqrt{3} \times 22)$-reconstruction. Examples are $\mathrm{Ag}[249,250], \mathrm{Cu}$ [251], and $\mathrm{Al}$ which we will discuss below. ${ }^{41}$ Meyer et al. noted that the elements displaying preferential nucleation at elbows all have a higher surface free energy than Au and a higher heat of sublimation. This energy balance is reversed for elements that show homogeneous nucleation (see table in Fig. 22). Within this simple energetic argument $\mathrm{Fe}, \mathrm{Co}$, and Ni should perform site selective place exchange (lower surface free energy) with subsequent preferred attachment for substitutional islands (higher heat of sublimation), yielding a one-to-one correlation of nucleation sites with the surface reconstruction. The other metals mentioned here have no tendency for intermixing and therefore undergo homogeneous nucleation. These energetic arguments are very tempting as they explain all experimental results obtained so far. Whether they capture the central point, i.e. whether exchange is indeed always active when islands are located at elbows, has to be investigated. A case where the system does not follow the energetic arguments is $\mathrm{Al} / \mathrm{Au}\left(\begin{array}{lll}1 & 1 & 1\end{array}\right)$ which we will now address.

\footnotetext{
${ }^{41}$ The case of $\mathrm{Au}(111)$ auto-epitaxy is less clear. In a study by Chambliss et al. [242] too few islands formed at room temperature precluding statistically significant statements. In contrast, an earlier work found nucleation at elbows. In the earlier study, however, the sample was transferred through air, which is likely to have invoked contamination problems [252].
} 


\subsubsection{Adatom diffusion on Au(1 11 1) - the effect of dislocations}

From a comparison of the surface free energies $\mathrm{Al}$ is expected to stay on $\mathrm{Au}(1111)$. This is indeed the case, but only for low temperature nucleation, and this is only because of the elevated activation energy for Al-Au exchange. Above $245 \mathrm{~K}$ surface alloy formation is observed [253,254]. Here we focus on the pure adatom regime below the critical temperature for exchange. This permits investigation of the effect of the partial dislocations on adatom diffusion without the complication brought about by exchange. From the STM image shown in Fig. 23 it is seen that at $150 \mathrm{~K} \mathrm{Al}$ islands nucleate in-between the partial dislocations of the unperturbed Au reconstruction. In the Arrhenius plot of the saturation island density there are three regimes of nucleation, each one clearly distinguished by its slope. The change in slope at $245 \mathrm{~K}$ is interpreted as reflecting reduced $\mathrm{Al}$ adatom mobility on the surface alloy. The second change in slope is located at $200 \mathrm{~K}$ deposition temperature and at an island density corresponding to one island within the $(\sqrt{3} \times 22)$-unit cell of the reconstruction (see dashed horizontal line). Below $200 \mathrm{~K}$ the island densities lie above that value. The mean island distances are smaller than the distance between dislocations, and the mean free path of diffusing atoms also being below this value, the adatoms are not influenced much by the dislocations. Above $200 \mathrm{~K}$, the mean inter-island distance lies above the period of 22 bulk atoms suggesting that adatoms diffused across dislocations. At the same time, the densities follow a slope whose gradient is 16 times steeper than in the $T<200 \mathrm{~K}$ regime. As the Au reconstruction stays unperturbed up to its transformation into a surface alloy at

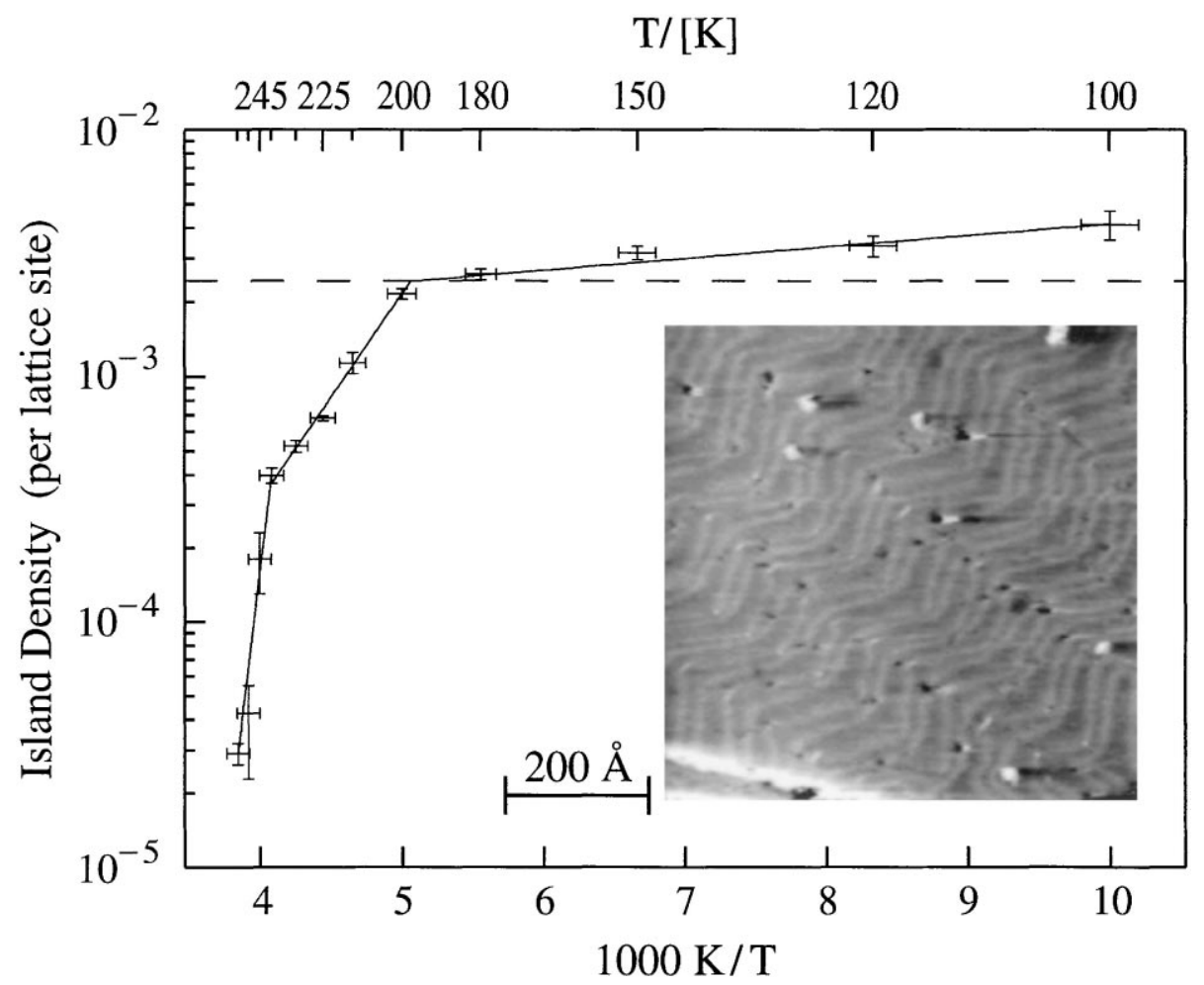

Fig. 23. Arrhenius plot of saturation island densities deduced from variable-temperature STM measurements for the nucleation of $\mathrm{Al}$ on $\mathrm{Au}\left(\begin{array}{lll}1 & 1 & 1\end{array}\right)-(\sqrt{3} \times 22)\left(\Theta=0.10-0.15 \mathrm{ML}, F=3.1 \times 10^{-4} \mathrm{ML} / \mathrm{s}\right)$. The inset shows that islands nucleate in between the partial dislocations $\left(\Theta=0.04 \mathrm{ML}, T=150 \mathrm{~K}\right.$, and $\left.F=3.1 \times 10^{-4} \mathrm{ML} / \mathrm{s}\right)$ (from Ref. [190]). 
$245 \mathrm{~K}$, the change in diffusivity characterizes pure adatom diffusion on the $(\sqrt{3} \times 22)$-reconstructed surface. As there was no preference for nucleation at any particular site of the reconstruction, and as most of the $\mathrm{Al}$ islands were located in the pseudomorphic stacking areas, the change in slope was attributed to a strong repulsion of adatoms at dislocations.

The discovery that dislocations can represent repulsive barriers is corroborated by EMT calculations performed for $\mathrm{Pt} / \mathrm{Pt}\left(\begin{array}{lll}1 & 1 & 1\end{array}\right)$ [255]. In these calculations the binding energy of the $\mathrm{Pt}$ adatom is found to decrease as it approaches the partial dislocation. The change in total energy is, however, small (half the migration barrier). The calculations further show that the increase in potential energy of the adatom is a long range effect extended over \pm 4 lattice sites adjacent to the partial dislocations. This long range effect implies that atoms have to make several successive jumps in energetically unfavorable directions to overcome a dislocation. This might explain high effective barriers although the total change in binding energy is relatively small.

A quantitative analysis of the two slopes in Fig. 23 in terms of activation energies for Al diffusion on pseudomorphic areas and across dislocations is not trivial. As we will see below, mean-field nucleation theory in its classical form for isotropic substrates is no longer applicable for nucleation on inhomogeneous substrates. A quantitative description therefore necessitates realistic KMC simulations [190]. In these simulations barriers for moving up dislocations are larger than those for descending from dislocations. The barrier for diffusion within the pseudomorphic stacking areas enters as third parameter. According to the EMT results for Pt/Pt [255], these barriers are selected within a certain range around the dislocations (typically \pm 4 atomic distances). The behavior of the island densities below and above $200 \mathrm{~K}$ can be reproduced by these simulations [190]. The activation energy for Al diffusion on pseudomorphic $\mathrm{Au}(111)$ emerges to $E_{\mathrm{m}}=35 \pm 5 \mathrm{meV},{ }^{42}$ and rather large effective barriers are obtained for crossing of dislocations (the total energy difference is $540 \mathrm{meV}$ for an $\mathrm{Al}$ atom on top of a dislocation compared to one on the pseudomorphic areas). The small barrier for Al diffusion on pseudomorphic Au(1 11 1) compares well with results obtained for Al(1 111 ) self-diffusion from ab initio calculations by Stumpf and Scheffler [132] yielding a barrier of $40 \mathrm{meV}$. However, much higher

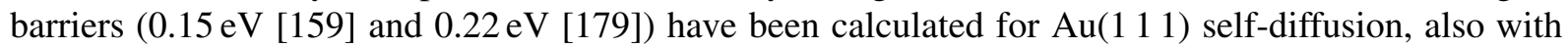
first principles methods. The large effective repulsion of diffusing adatoms at dislocations is in line with their strong influence on island densities and film growth observed in other epitaxial metal systems $[151,232]$.

\subsubsection{Nucleation on dislocation networks}

The second Ag layer adsorbed at room temperature onto $\mathrm{Pt}\left(\begin{array}{lll}1 & 1\end{array}\right)$ forms a striped incommensurate phase with pairs of partial dislocations oriented along $\left\langle\begin{array}{lll}1 & 1 & 2\end{array}\right\rangle$. In analogy to the $\mathrm{Au}\left(\begin{array}{lll}1 & 1 & 1\end{array}\right)$ reconstruction and the $\mathrm{Cu}$ bilayer on $\mathrm{Ru}(0001)$ [256,257], strain relief in this structure can only be unidirectional on a local scale. For $\mathrm{Ag} / \mathrm{Pt}\left(\begin{array}{lll}1 & 1 & 1\end{array}\right)$ this structure turns out to be metastable. Upon annealing to $800 \mathrm{~K}$, it transforms into a trigonal network of crossing dislocations [155]. In this way, isotropic strain relief is achieved on a small length scale. With this transformation the population of sites also changes to favor fcc stacking [156], as expected from the generally higher binding energy of these sites [158]. The larger amount of fcc areas is achieved through displacement of one class of parallel domain walls by an offset

\footnotetext{
${ }^{42}$ Note the extremely small attempt frequency of $\nu_{0}=10^{4 \pm 1} \mathrm{~s}^{-1}$, see Section 3.6. Identical values for the migration barrier and attempt frequency are extracted from the slope for $T<200 \mathrm{~K}$ upon application of mean-field theory for isotropic diffusion. This indicates that in this regime the dislocations have only minor influence and isotropic nucleation theory is applicable.
} 
a)

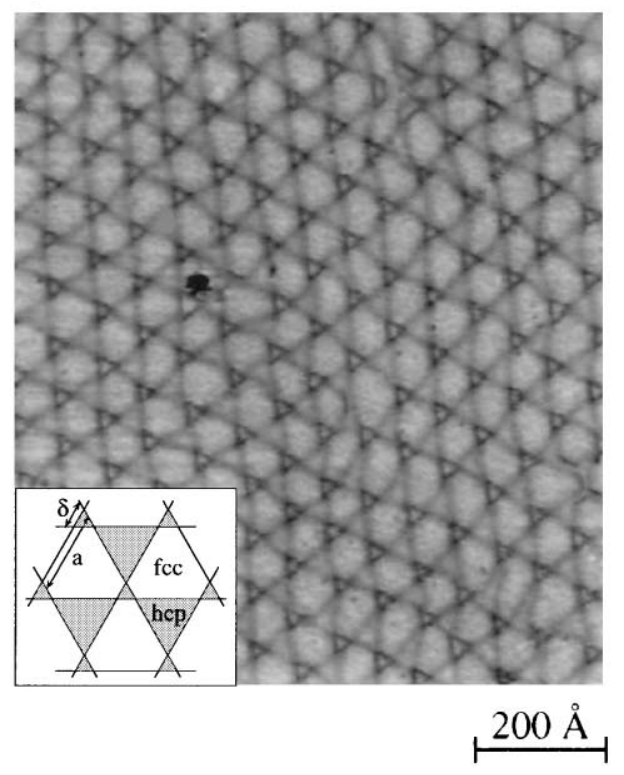

c)

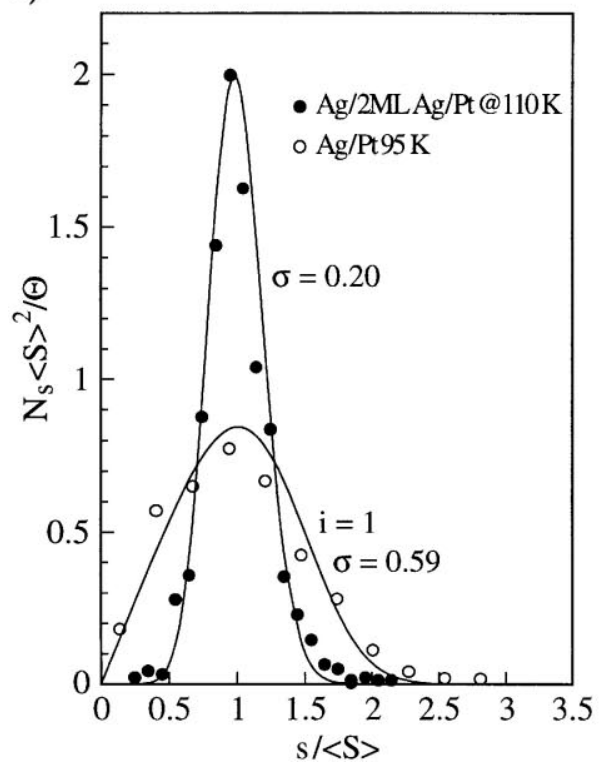

b)

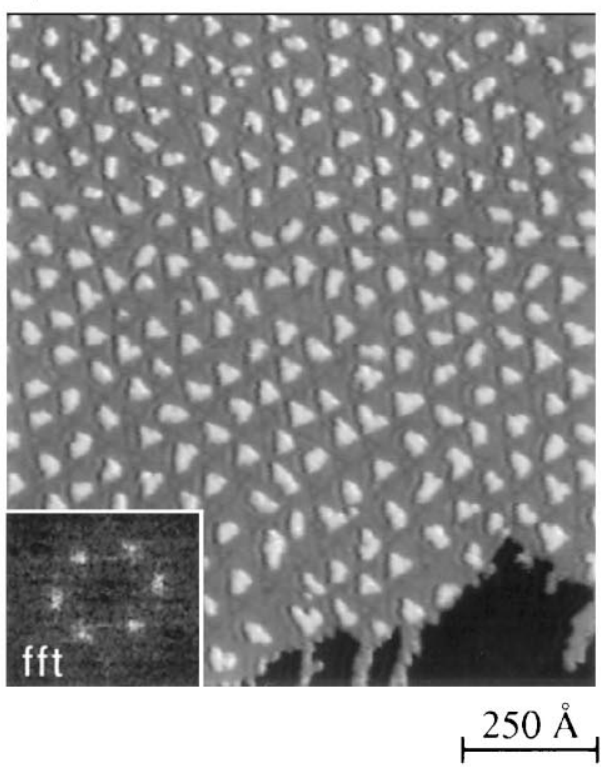

d)

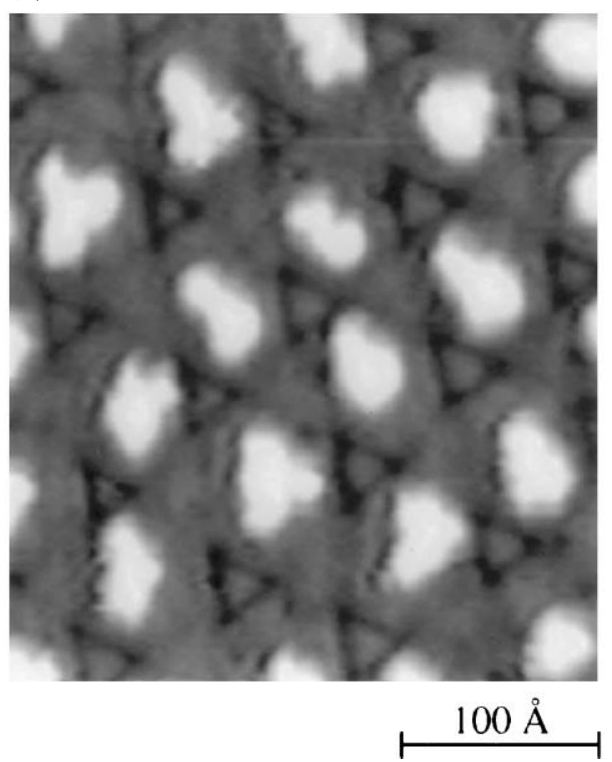

Fig. 24. Nucleation of an island superlattice on a reconstruction network. (a) STM image of the domain wall network formed by the second $\mathrm{Ag}$ monolayer on $\mathrm{Pt}\left(\begin{array}{lll}1 & 1 & 1\end{array}\right)$. The inset shows a model of the trigonal strain relief pattern. (b) Ag nucleation on this network at $T=110 \mathrm{~K}$ yields an island superlattice with exactly one island per superstructure unit cell $(\Theta=0.10 \mathrm{ML})$. Inset: fft of the STM image. (c) Almost monodispersed size distributions are associated with the periodic island spacing. (d) Detail showing that islands form exclusively on the fcc stacking areas (from Ref. [235]). 
( $\delta$ in the model in Fig. 24(a)) relative to the crossing point of the two others. This leads to a dislocation network with trigonal symmetry. By adjusting the offset $\delta$, the system can optimize the ratio between fcc and hep stacking [29]. Crossing of three dislocations at a single point is energetically costly as it involves on-top sites. The offset allows this situation to be avoided. Due to long range interactions between dislocations, long range order of the network is established as apparent from the STM image reproduced in Fig. 24(a).

Ag nucleation on top of this network at low temperature leads to a high density of islands where the majority are located away from dislocations [150]. This implies that dislocations constitute repulsive barriers for diffusing adatoms, as in the preceding example of $\mathrm{Al} / \mathrm{Au}(111)$. For increasing temperatures the island density approaches a stationary value of exactly one island per network unit cell at $110 \mathrm{~K}$ (see Fig. 24(b)). Note that all of the islands nucleate on the distorted hexagons of the unit cell (see Fig. 24(d)). This implies preferential binding to these fcc stacking areas [158]. As a consequence dislocations present a smaller energy barrier for atoms coming from hcp areas than for those coming from fcc stacking areas (see Fig. 25(c)). Thus, atoms landing in one of the two hcp triangles within the unit cell can diffuse into the fcc hexagons but not vice versa. Due to the repulsive nature of the dislocations and the attraction towards specific sites within the unit cell the periodicity of the dislocation network is congruently transferred to an island superlattice. The high degree of order and the hexagonal symmetry of the island array become evident through sharp peaks in the Fourier transform of the STM image (see inset Fig. 24(b)).

In general there is a correlation between island spacings and island sizes [258,259]. Material deposited onto the area closest to an island is likely to attach to that island. This capture area of an island is its Voronoi polygon. For equidistant islands these areas are identical therefore leading to very narrow island size distributions. This is exactly the situation under examination here. In our example the Voronoi areas are the $(25 \times 25)$ network unit cells. The scaled island size distribution (Fig. 24(c)) is accordingly significantly sharper than that obtained for homogeneous nucleation. We note that there is a residual width due to tip convolution. Therefore, the measured standard deviation of $\sigma=0.20$ represents an upper bound. The theoretical lower bound for $\sigma$ is given by the statistics of deposition. Consider the ideal case of infinite barriers at dislocations such that adatoms stay confined within the unit cells and form one island there. For random deposition of an average coverage $p$ into unit cells with size $n$, the probability of finding $k$ atoms within a unit cell obeys a binomial distribution [235]. This distribution, when normalized according to Fig. 24(c), has a standard deviation of $\sigma=\sqrt{q / n p}$. For our case of $p=0.1, q=0.9$ this yields $\sigma=0.12$ for $(25 \times 25)$ unit cells $(n=625)$. This result from confined nucleation compares favorably to size distributions currently obtained in self-organized growth of quantum dots which have a standard deviation of 0.16 in surface area [260]. From the considerations above it can be inferred that $\sigma$ decreases for larger unit cells and larger coverages (due to reduced fluctuations). For example, deposition of $0.2 \mathrm{ML}$ into $50 \times 50$ unit cells leads to extremely sharp island size distributions with $\sigma=0.04$. These values refer to island areas; the distribution of island diameters has only half this width.

Quantitative understanding of nucleation on this superlattice is achieved through analysis of the Arrhenius behavior of the saturation island density (Fig. 25(a)). There are two slopes separated by a plateau at a density of one island per superstructure unit cell. ${ }^{43}$ For temperatures below this plateau, the

\footnotetext{
${ }^{43}$ This Arrhenius behavior is also obtained for nucleation in the presence of trapping defects [58,261]. The authors of Refs. $[58,261]$ show how defect nucleation can be incorporated into mean-field nucleation theory.
} 
a)

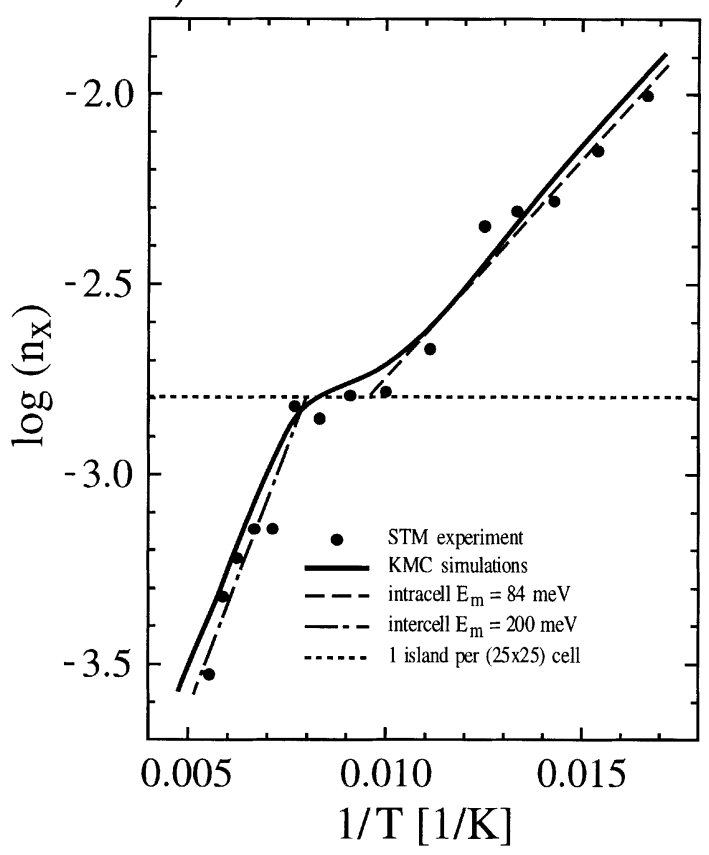

c)

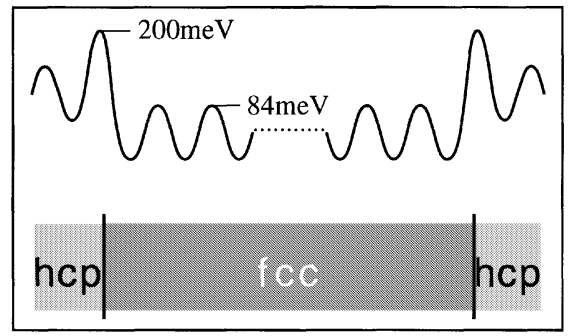

b)

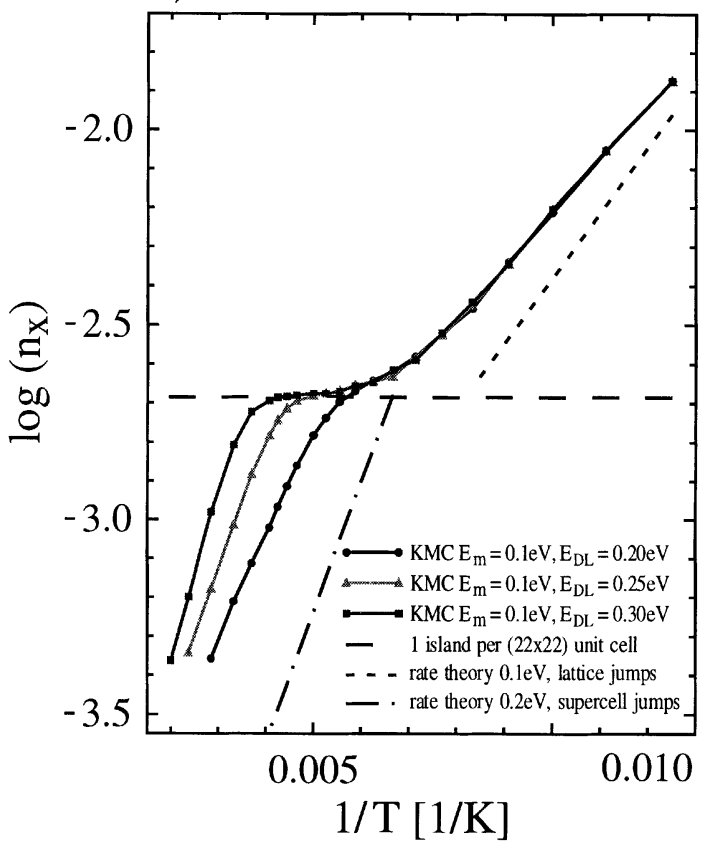

Fig. 25. Crossover scaling for nucleation on a periodic network with repulsive dislocations. (a) Arrhenius plot of experimental island densities for the system shown in Fig. 24 in comparison with KMC simulations of which the parameters were adopted to that system. (b) KMC for the general case of nucleation on a hexagonal network. Deviations from isotropic scaling are evident. (c) Model showing that the real system reveals larger adsorption energies on fcc as compared to hcp stacking areas (from Ref. [235]).

mean free path of Ag adatoms is smaller than the $(25 \times 25)$ unit cell, leading to nucleation of several islands per unit cell. At temperatures within the plateau, the diffusivity becomes sufficient for the adatoms to visit the entire unit cell. Their thermal energy not yet suffices to surmount the barrier represented by the dislocations, however. As a result the adatoms stay confined within the unit cell into which they were deposited and nucleate one island there. Eventually, the barrier at dislocations can be overcome and the island density further decreases. The first slope therefore characterizes diffusion within the unit cell, the second diffusion across dislocations. 
The system can be described very well by the transition between two rate limiting processes, i.e. from inter-cell diffusion over one lattice site to intra-cell diffusion over $(25 \times 25)$ lattice sites. The island densities expected from nucleation theory for both cases are shown as dashed and dash-dotted lines in Fig. 25(b). From comparison to the KMC results performed for such a lattice (barrier within the unit cells $E_{\mathrm{m}}=0.1 \mathrm{eV}$ and barriers to cross dislocations $E_{\mathrm{DL}}=0.2-0.3 \mathrm{eV}$ ), the actual island densities are seen to lie above both regimes. This is expected as a system combining two rates is generally slower than the corresponding cases with only one process. In addition, the slope at low $D / F$ is smaller than would be expected from diffusion on an isotropic substrate. The adatoms "see" the dislocations although they cannot overcome them and correspondingly scaling laws change. As expected, the barrier chosen for dislocations has no influence on the island density within the unit cell, as long as it cannot be overcome on the timescale of deposition. The length of the plateau is determined by the barrier at dislocations. Finally, the island densities beyond the plateau show the different slopes corrsponding to these barriers, no matter what the barrier in the unit cell was.

From these simulations agreement with experiment is obtained for $E_{\mathrm{m}}=0.084 \pm 0.008 \mathrm{eV}$ and $E_{\mathrm{DL}}=0.20 \pm 0.03 \mathrm{eV}$ (see full curve in Fig. 25(a)). The preference for nucleation on fcc sites was accounted for by introducing only two classes of dislocations in the simulations rendering each unit cell a simple rhombus. The energy barrier for diffusion on the pseudomorphic stacking areas of the second Ag layer is greater than that measured on the pseudomorphic first layer and less than that on $\operatorname{Ag}\left(\begin{array}{lll}1 & 1 & 1\end{array}\right)$. This is what we expect as in these patches of the second layer the compressive strain is presumably less than in the first layer and at the same time it is not entirely relieved.

\subsubsection{Periodic superlattices suited for self-ordering}

Periodic arrangements of dislocations are quite often found in epitaxial systems. Trigonal networks of the type just described are found in a number of epitaxial systems. Examples for metals are $\mathrm{Na}$ adsorption on $\mathrm{Au}\left(\begin{array}{lll}1 & 1 & 1\end{array}\right)$ [262], $\mathrm{Ni}$ on $\mathrm{Ru}\left(\begin{array}{llll}0 & 0 & 0 & 1\end{array}\right)$ [151], or $\mathrm{Cu}$ on the same surface [257,263]. There are also examples of regular dislocation networks in semiconductors, for example the $\beta$-phase of $\mathrm{Ga}$ on Ge(1 11 1) [264,265], or surfactant mediated growth of Ge on $\operatorname{Si}\left(\begin{array}{lll}1 & 1 & 1\end{array}\right)$ [266].

Moiré structures are formed by adlayers where the lateral interaction is stiff compared to the corrugation of the substrate potential. They reveal periodic variations in strain and/or adatom binding energy. This makes them feasible candidates for periodic diffusion anisotropies that can give rise to island networks. ${ }^{44}$ Many examples for such moiré patterns exist: graphite monolayers on $\operatorname{Pt}\left(\begin{array}{lll}1 & 1 & 1\end{array}\right)$ [267]; sulfides [268]; metal overlayer systems such as $\mathrm{Ag} / \mathrm{Cu}\left(\begin{array}{lll}1 & 1 & 1\end{array}\right)$ and $\mathrm{Ag} / \mathrm{Ni}\left(\begin{array}{lll}1 & 1 & 1\end{array}\right)$ [28]; and, finally metal on semiconductor systems like $2 \mathrm{ML} \mathrm{Cu} / \mathrm{Ge}\left(\begin{array}{lll}1 & 1 & 1\end{array}\right)$ [269]. These moirés have the superstructure periodicity $d=a_{\mathrm{ad}} /\left(a_{\mathrm{ad}}-a_{\mathrm{sub}}\right)$ determined by the adlayer and substrate lattice constants (the formula is valid for the non-rotated case).

For the purpose of growing island superlattices particularly interesting examples of periodic strain relief patterns are those where we can adjust the size of the unit cell and with it the island spacing by an external parameter. An example is the surface alloy formed by $\mathrm{Au}$ on $\mathrm{Ni}\left(\begin{array}{ll}1 & 1\end{array}\right)$ above room temperature $[28,270]$. Here the Au coverage determines the dimension of the periodic superstructure. STM topographs of $\mathrm{Au}$ adsorbed onto $\mathrm{Ni}$ at room temperature reveal monoatomically high islands that contain regularly space triangles (see Fig. 26(a)). The Au atoms forming the triangle border are imaged

\footnotetext{
${ }^{44}$ First results show that superlattices of magnetic elements $(\mathrm{Fe})$ can be grown upon deposition onto a moiré pattern formed by $2 \mathrm{ML} \mathrm{Cu}$ on $\mathrm{Pt}\left(\begin{array}{lll}1 & 1 & 1)\end{array}\right.$ [235].
} 
a)
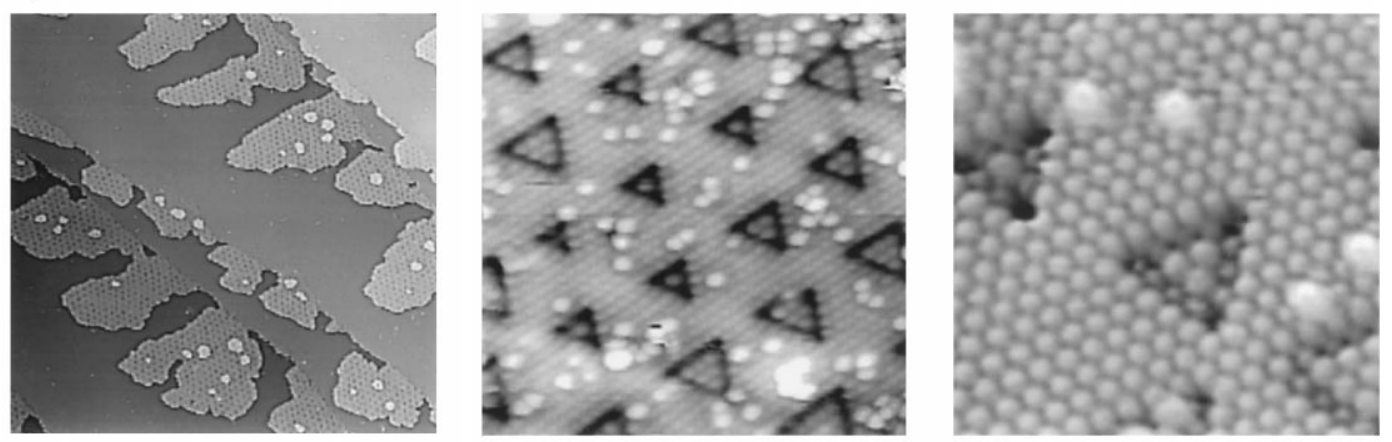

b)

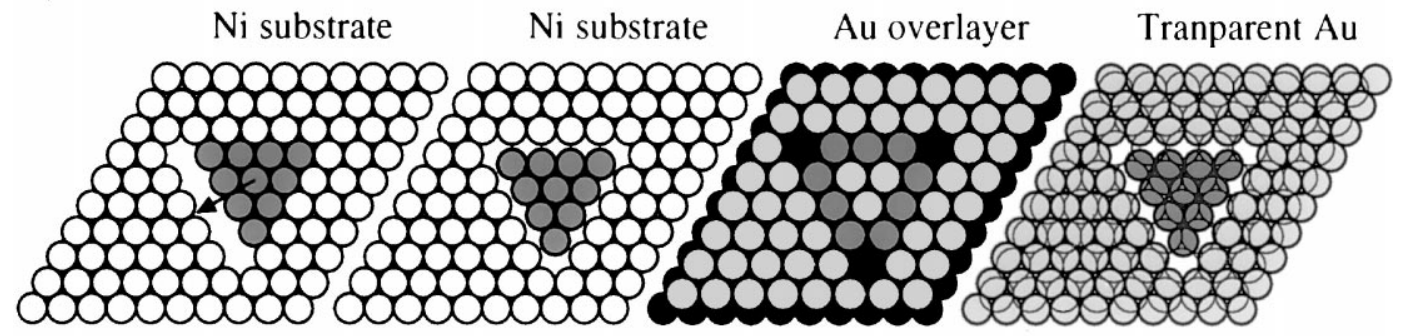

c)
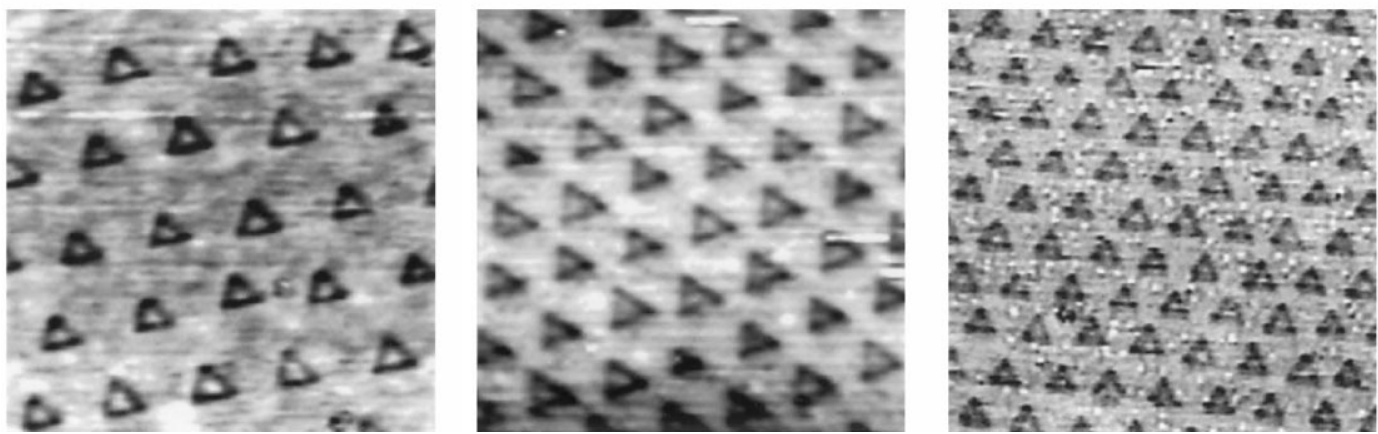

Fig. 26. Regular network of dislocations with adjustable lattice constant: Au/Ni(1 11 1). (a) STM images after deposition of $0.25 \mathrm{ML} \mathrm{Au}$ at RT. From left to right the structure in the Au islands is increasingly zoomed into, revealing the Au triangles formed by the DL loop of the underlying Ni. The bright atoms are Ni atoms kicked out of the substrate into the Au level (left $1450 \times 1570 \AA^{2}$, center $100 \times 100 \AA^{2}$, right $45 \times 50 \AA^{2}$ ). (b) Model showing the Ni DL loop which is subsequently covered with the $\mathrm{Au}$ layer. In the right-hand figure, the $\mathrm{Au}$ atoms are made transparent revealing that all $\mathrm{Au}$ atoms are located on threefold hollows (from Refs. [174,270]). (c) STM images $\left(200 \times 200 \AA^{2}\right)$ after deposition of Au at $700 \mathrm{~K}$ again revealing a network of triangles. This time, however, they are formed by a Au-Ni alloy layer residing on DL loops of the underlying Ni. The network period can be adjusted through the Au coverage following Vegard's law in 2D. From left to right: unit cell size $(20 \times 20)$ at $\Theta_{\mathrm{Au}}=0.35 \mathrm{ML},(13 \times 13)$ at $\Theta_{\mathrm{Au}}=0.50 \mathrm{ML}$, and $(10 \times 10)$ at $\Theta_{\mathrm{Au}}=0.70 \mathrm{ML}$ (from Ref. [270]). 
deeper than those which make up the unperturbed lattice in-between the triangles. Both the Au atoms within the troughs and those in the triangle center are in registry with the overall Au lattice, ruling out dislocations within the adlayer. An explanation of the structure by Jacobsen et al. [174] proposes vacancy dislocation loops in the Ni underneath. As displayed in the model Fig. 26(b), Ni atoms are squeezed out of the first Ni layer into the Au layer, where by their imaging characteristics they become visible as white protrusions in the STM topographs (Fig. 26(a)). The energetic motivation for the dislocation loops in the underlying substrate is that Au atoms can be in or close to three-fold sites while keeping the preferred lateral distance, as opposed to energetically unfavorable on-top sites implied by a moiré overlayer, for example. That way the interfacial strain is reduced through the introduction of misfit dislocations in the first atomic layer of the substrate. Confirmation of this triangular misfit model comes from the one-to-one correspondence between the number of $\mathrm{Ni}$ atoms squeezed out of the substrate in order to form the dislocation loops and the number of $\mathrm{Ni}$ atoms found to be alloyed into the $\mathrm{Au}$ layer [270]. The structure also represents the minimum energy configuration in EMT calculations [174].

As the temperature is increased, the Au-rich islands dissolve and a homogeneous surface alloy between $\mathrm{Ni}$ and $\mathrm{Au}$ is formed. As this surface still reveals the dislocation loops in the underlying $\mathrm{Ni}$ substrate, the triangles prevail (see Fig. 26(c)). These might represent preferred nucleation sites for an island superlattice. The important point is that the period of the superlattice depends on the amount of $\mathrm{Au}$ deposited. The network unit cell monotonically decreases from $(20 \times 20)$ at $0.35 \mathrm{ML} \mathrm{Au}$ to $(10 \times 10)$ at $0.70 \mathrm{ML} \mathrm{Au}$. The reason for this adjustment is the concentration dependence of the effective atomic lattice constant $a_{\text {eff }}$ within the 2D alloy. A variation of $a_{\text {eff }}$ with concentration is well known from bulk binary alloys as Vegard's law [271]. Applying this law to surfaces yields a coverage dependent effective 2D lattice constant which, in turn, quantitatively explains the measured variation of the lattice constant in terms of a moiré superstructure formed by $a_{\mathrm{eff}}$ and the underlying $a_{\mathrm{Ni}}[28,270]$. Nucleation studies on alloyed surfaces which reveal such superlattices promise to yield equidistant islands through the link between surface inhomogeneity and adatom diffusion.

Whereas the networks discussed so far constitute possible templates for the growth of island superlattices, we now turn to a substrate with unidirectional periodicity, which provides a candidate substrate for the creation of regularly spaced 1D structures. In the example of $\mathrm{Cu} / \mathrm{Pd}\left(\begin{array}{lll}1 & 1 & 0\end{array}\right)$ discussed in Section 4.1, 1D strings of atoms are formed. However, their perpendicular spacing is given by the randomness of coventional nucleation. This may be different on substrates where strain induced lateral interactions provide troughs with similar spacing. An example is $\mathrm{Pb}$ adsorbed on $\mathrm{Cu}(110)$ at room temperature. For coverages above $0.4 \mathrm{ML}$ the $\mathrm{Pb}$ adphase transforms from a $2 \mathrm{D}$ lattice gas into several well-ordered condensed phases. As in the above example, the periodicity of these different phases depends on coverage and can therefore be tuned within certain limits. At $0.5 \mathrm{ML}$ a $\mathrm{c}(2 \times 2)$ phase forms [272-274]. This phase transforms into $(n \times 1)$ structures, where $n$ ranges from 2 to 13 for $0.7 \mathrm{ML}<\Theta_{\mathrm{Pb}}<0.8 \mathrm{ML}$ [272]. The long range order formed by $0.75 \mathrm{ML} \mathrm{Pb}$ on $\mathrm{Cu}(110)$ is characterized by a regular spacing of troughs running along the $\langle 001\rangle$-direction (see Fig. 27(a)). The detail in Fig. 27(b) reveals the atomic structure consisting of a combination of $(8 \times 1)$ and $(4 \times 1)$ unit cells. The atoms are commensurate with the $\mathrm{Cu}$ substrate in its $\langle 001\rangle$-direction. Along the closepacked rows, the difference in atomic radii leads to superstructures that depend on coverage, as does the mean interatomic $\mathrm{Pb}$ distance. One of the $(4 \times 1)$ structures involves a rearrangement of the underlying $\mathrm{Cu}$ substrate similar to the case $\mathrm{Au} / \mathrm{Ni}\left(\begin{array}{lll}1 & 1 & 1\end{array}\right)$. One atomic row along $\langle 001\rangle$ is missing such that $\mathrm{Pb}$ atoms can dive into the grooves. That way the system increases the adlayer substrate binding which 
a)

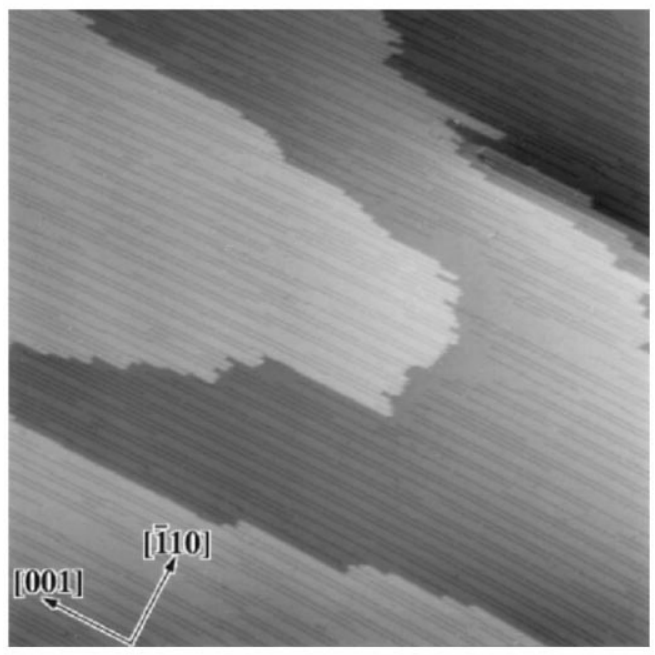

b)

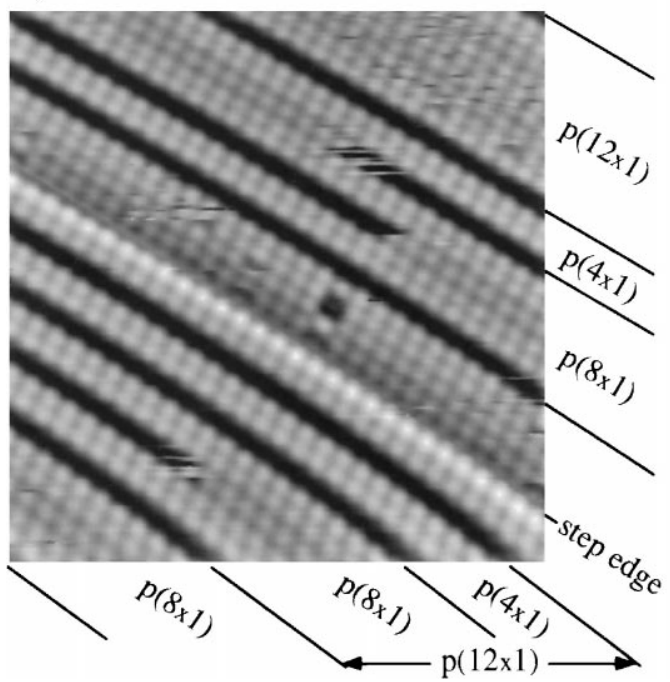

c)
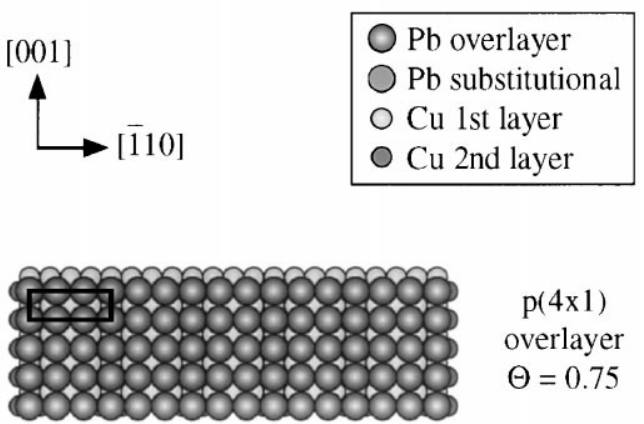

$\mathrm{p}(4 \times 1)$

overlayer

$\Theta=0.75$

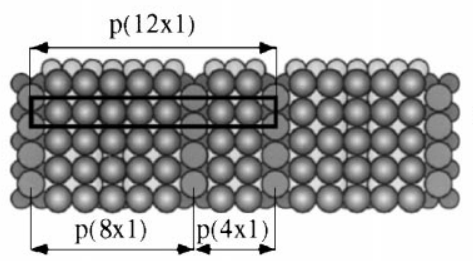

$\mathrm{p}(12 \times 1)$

substitutional

+ overlayer

$\Theta=0.75$

$p(4 \times 1)$

substitutional

$\Theta=0.75$

$\mathrm{p}(4 \times 1)$

substitutional

side view

Fig. 27. Possible substrate for the growth of equally spaced $1 \mathrm{D}$ structures: $0.75 \mathrm{ML} \mathrm{Pb}$ adsorbed onto $\mathrm{Cu}(110)$ at $300 \mathrm{~K}$. (a) STM image showing an overview where the regularly spaced grooves are oriented along [001]. (b) Detail revealing the atomic structure of the $\mathrm{p}(n \times 1)$ phases $(n=4,8,12)$. The grooves are formed by substitutional atoms. (c) Ball model of the $(4 \times 1)$ overlayer and the substitutional phases (size of STM figures: (a) $1000 \times 1000 \AA^{2}$; and (b) $100 \times 100 \AA^{2}$ ) (from Ref. [272]). 
overbalances the cost of removing a substrate row. ${ }^{45}$ The resulting troughs at the surface are well localized and can serve as diffusion channels to built up equally spaced 1D islands by self-organized growth.

The examples discussed in this section illustrate that dislocations in general strongly influence nucleation. They can constitute rather effective repulsive barriers, by which they dominate the kinetics of heteroepitaxy. In addition, this property of dislocations may be employed to grow island superlattices on well-ordered dislocation patterns. A quantitative understanding of diffusion close to and across dislocations or repulsive point defects, in general of diffusion on inhomogeneous substrates, is indispensable for a better understanding of self-ordering processes at surfaces and of the kinetics in heteroepitaxial growth.

\section{Aggregation and pattern formation at surfaces}

The fascinating growth patterns observed in nature are commonly formed at interfaces and under non-equlibrium conditions where material transport via diffusion is the growth determining process. Exactly these conditions govern aggregation at single crystal surfaces. Aggregation of metal atoms (in the submonolayer range) is strictly $2 \mathrm{D}$, and therefore, considerably easier to understand than many complex growth patterns in nature. This makes low temperature metal aggregation to an ideal model system where the mechanisms leading from single diffusion events of the atomic building blocks to the overall pattern shape can be traced back. Access to the structure of aggregates formed by atoms at surfaces was opened up by the development of temperature controlled scanning tunneling microscopy. Following this development pattern formation was extensively investigated for metal on single crystal metal surfaces [17,92,101,279-282]. These studies revealed many details of how atomic diffusion at or close to an aggregate's edge can determine the ultimate mesoscopic shape. The results of these very recent research activities will be reviewed in this section. Special emphasis will be put on the comparison with other examples for patterns in nature with the goal to show up similarities encouraging that knowledge gained on the atomic scale for these highly ideal systems may be transferred to the understanding of other non-equilibrium growth patterns in nature.

\subsection{Pattern formation in nature and modeling attempts}

\subsubsection{Non-equilibrium patterns and their common characteristics}

The similarity of patterns formed in non-equilibrium growth processes in physics, chemistry and biology is conspicuous, and many attempts have been made to discover common mechanisms underlying their formation [13,14]. The most prominent examples of growth patterns in nature are snowflakes which reveal a fascinating degree of order (see Fig. 28(a) and Ref. [283]). Snowflakes have fascinated mankind for centuries. It was Kepler [284] who tried first to explain the macroscopic symmetry of snow crystals by examining the manner in which the elementary building blocks might

\footnotetext{
${ }^{45}$ In the $(8 \times 1)$-phase there is a trough where $\mathrm{Pb}$ atoms reside in substitutional sites. In addition, the row of $\mathrm{Pb}$ adatoms in the center of the unit cell lies slightly deeper than the rest of the $\mathrm{Pb}$ atoms. Although a detail $(0.02 \AA)$, this height is unexpected as these atoms reside on bridge sites. It can be explained, however, through relaxation of the substrate [275]. Substrate relaxation often leads to adsorption heights in the topmost layer that are unexpected when assuming a rigid substrate $[155,164,275-278]$.
} 
a)

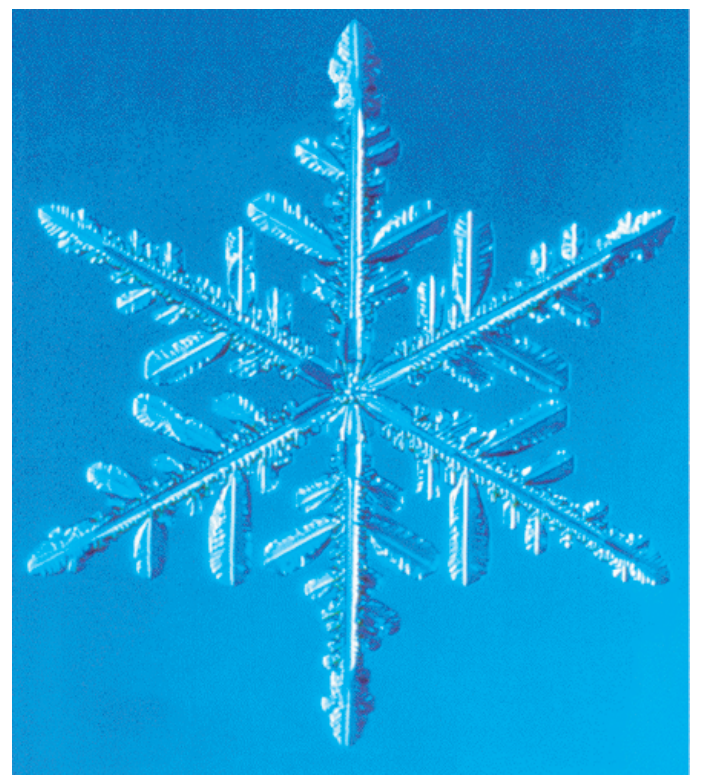

c)

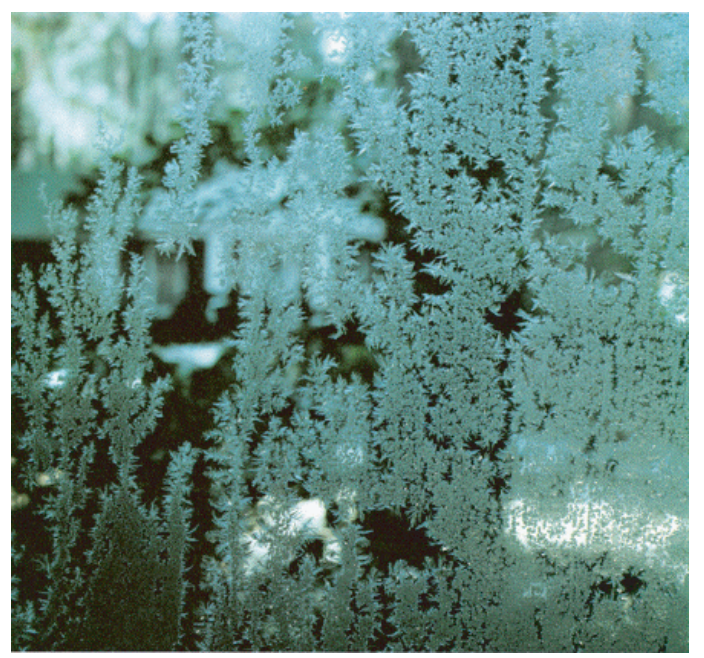

b)

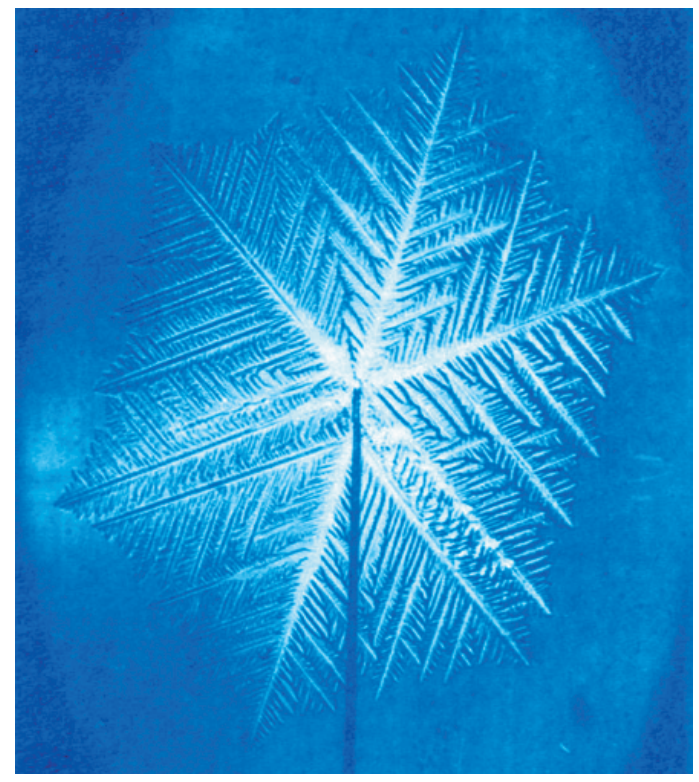

d)

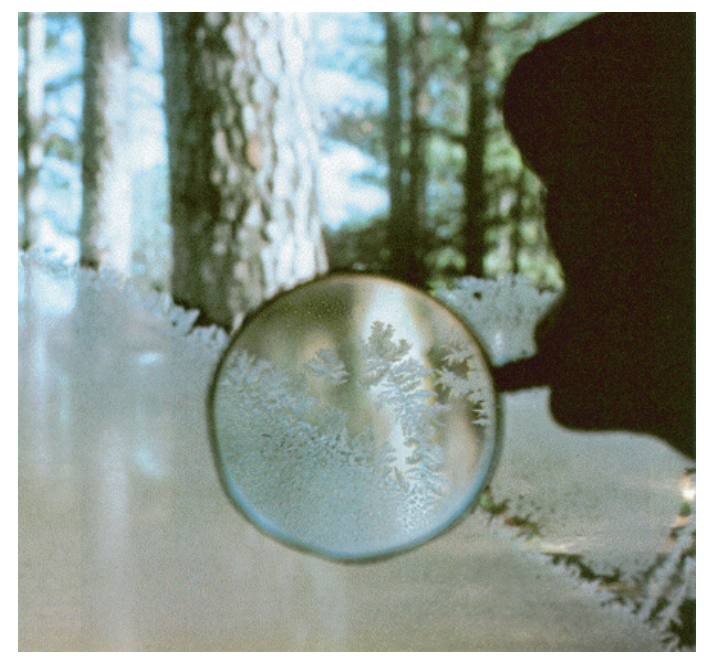

Fig. 28. (a) Snowflake (from Ref. [283]). (b) Dendritic ice crystal grown in pure undercooled water (from Ref. [289]). (c) Ice Øowers grown on a window, and (d) detail from image (c) revealing the self-similarity (from Ref. [290]). 
pack together. Descartes was also attracted by these highly structured objects [285,286]. An overview of the variety in shape of these objects was presented by Bentley in an impressive collection of photographs [287]. Later on, snowflakes could be classified into symmetry groups by Nakaya [288]. Nakaya [288] recognized that their shape bears information on the weather prevailing in upper strata of the atmosphere and this led him to the expression that snowflakes are "letters from the sky". The link between the morphology of a snow crystal and its growth conditions, as temperature and supersaturation, remained an open issue for almost 50 years, however. A breakthrough was achieved during the last 10 years showing that the laws of crystal growth are in fact strongly involved in the formation of snowflakes. Their growth starts from water droplets that crystallize into spherical ice particles of $10 \mu \mathrm{m}$ diameter bound by various crystal facets. Depending on the atmospheric conditions these facets have different growth speeds determining the final mesoscopic shape of snow crystals [283].

In addition to their symmetry snowflakes, similar to other non-equilibrium patterns, reveal spectacular mathematical properties. For example, they are self-similar. Zooming in onto an arbitrarily chosen detail reveals that the structure of the entire object repeats itself on a smaller scale. ${ }^{46}$ This property is illustrated in Figs. 28(c) and (d) which show ice flowers grown on a window. The detail in Fig. 28(d) closely resembles the overall pattern shape. This self-similarity means that the structure remains invariant under a change of the length scale. In particular, the correlation functions describing, for example, the snowflake's surface will have a scale-invariant (power law) form. The power appearing in these laws is a non-integer, fractal (the word was invented by Mandelbrot [291]) number smaller than the Euclidean dimension of the space in which the structure grows. This number gives the fractal dimension of the object. The fractal dimension was first defined by Hausdorff in 1919 [14]. There are several fractal measures. The most intuitive one, familiar from geometry, is the increase of volume with a non-integer power of the radius, the power being the fractal dimension. A fractal dimension can equally be inferred by analyzing the radius of gyration as a function of an aggregate's volume or the two point density-density correlation function [292]. For self-similar structures, all these fractal measures yield the same value, which is therefore called the "general purpose" fractal dimension. Only mathematical fractals exhibit an exact self-similar structure which can be extended from infinitely large to infinitesimally small length scales. For every fractal appearing in nature the range in which a power law with a fractal exponent can be established is necessarily limited towards small scale by the size of the building units, and by the aggregate's overall size at the other end of the length scale.

Further examples for non-equilibrium fractals that obey self-similarity within a certain length scale are ice crystals forming upon sudden solidification of undercooled water (see Fig. 28(b) and Ref. [289]), the growth patterns of bacterial colonies [293], or electric discharge patterns [294]. In addition, there are large scale objects known from earth sciences that similarly show fractal properties. Famous examples are the fractal shape of coastlines [295], a self-affine scaling exponent commonly exhibited by several mountain areas (for the Swiss landscape see Ref. [296]), and finally the fractal dimension characterizing rivers with their side branches like the Amazon. ${ }^{47}$

\footnotetext{
${ }^{46}$ In the mathematical sense, self-affine means that this self-similarity continues for an infinite number of iterations. Of course, only mathematical objects as a Sierpinski gasket (a hierarchical aggregation of triangles) can strictly obey this requirement. Objects in nature can, however, come rather close to ideal self-affinity.

${ }^{47}$ For an account of fractals in nature the reader may consult Refs. [13,14,297], a special survey on fractal surfaces is given in Refs. [298,299].
} 


\subsubsection{Shape transitions}

Symmetry is a fundamental property characterizing growth patterns in addition to self-affinity and fractal power laws. It is evident to the unaided eye that a snowflake is quite different from an electric discharge pattern. Whereas the first reveals (often six-fold) symmetry, the latter is entirely dominated by random growth. Nevertheless, both objects have fractal dimensions close to 1.7 indicating that symmetry is an additional property escaping from fractal measures.

a)

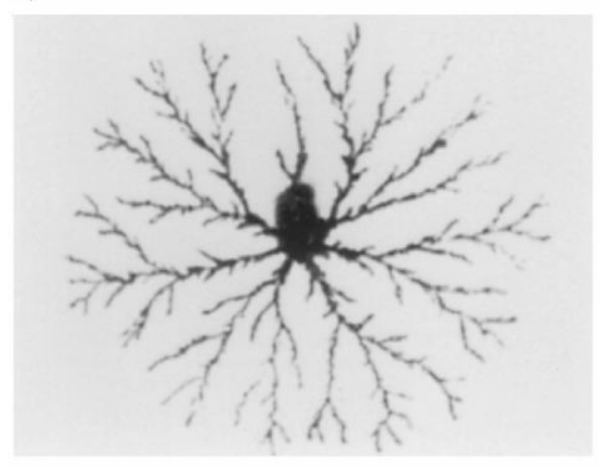

c)

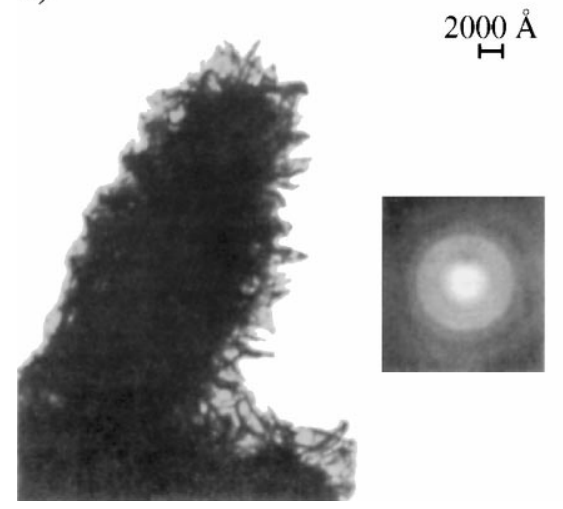

b)

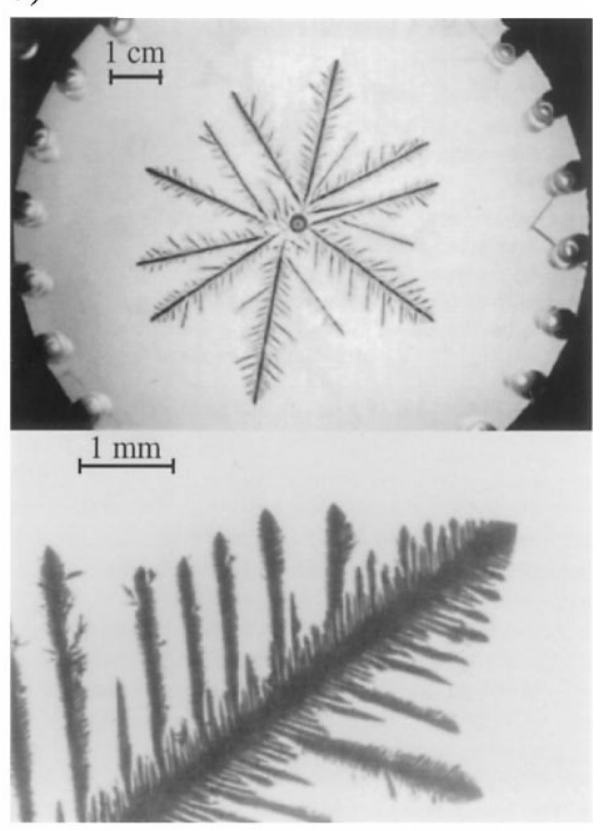

d)

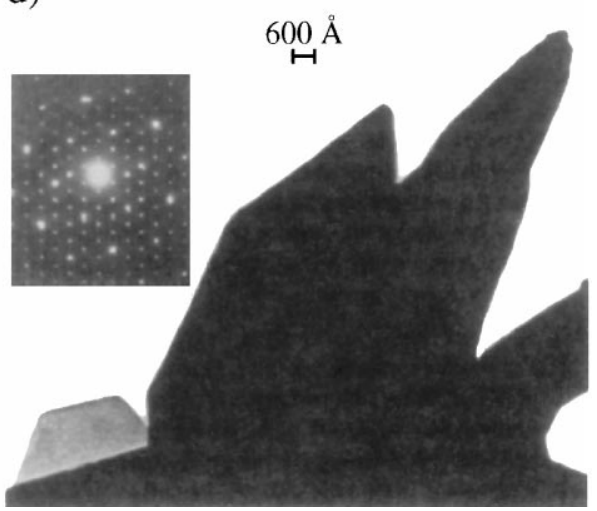

Fig. 29. Shape transition observed in electrochemical deposition of $\mathrm{Zn}$. With increasing growth speed random dominated growth transforms into dendritic growth. Dendrites have crystalline order apparent through sharp electron diffraction spots (from Refs. [300,301]). 
Very often, upon variation of the growth parameters one observes a transition from ordered symmetric growth towards random dominated growth within the same system. A typical example is crystal growth from the liquid. The electrochemical deposition of $\mathrm{Zn}$ is illustrated in Fig. 29. Increasing the growth speed $\left(\mathrm{Zn}^{2+}\right.$ concentration and voltage) results in the transition from random to ordered growth [300,301]. Whereas the branches grow in random directions at small growth rates (Fig. 29a), at higher deposition rates they are well oriented as in a needle crystal (Fig. 29b). The random aggregates reveal only short range order expressed in rings seen in electron diffraction (Fig. 29c), whereas the ordered patterns exhibit long range crystalline order manifested by sharp diffraction spots (Fig. 29d) [301]. In the following we will call the random patterns "fractals". Ordered symmetric patterns are commonly referred to as "dendrites".

Shape transitions also appear in a wide class of fluid dynamics experiments involving the propagation of a low viscous medium into a high viscous one. These experiments are typically performed in a Hele-Shaw cell [302]. Such a cell consists of a set of parallel glass plates which confine the high viscous medium (often being a liquid). In the center of one of the plates there is an inlet for the low viscous medium (this can be a gas or another non-miscible liquid). From certain propagation speeds on, viscous fingering occurs at the interface (Saffman-Taylor instability [303]). The dynamics are dominated by tip bifurcations which normally lead to randomly branched structures [304,305]. When anisotropy is introduced into these systems, either by scratching a lattice into one of the glass plates of the Hele-Shaw cell [305,306], or by use of a liquid crystal as high viscous medium [307], a transition from randomly ramified to dendritic patterns can be generated. In accord with the electrochemical deposition results discussed above, this transition takes place upon an increase of the expansion rate.

The growth of bacterial colonies exhibits similar shape transitions. These systems are equally limited by diffusion as the bacteria move in response to the gradient of the nutrient concentration. At high nutrient concentrations (large growth speeds) the colonies show symmetric ordering whereas they transform over randomly to dense ramified aggregates for decreasing nutrient concentration [293]. Bacterial colonies reveal cooperative behavior requiring local communication. This is a generic property, also for non-living growth patterns where this communication is established via the diffusion field. Neighboring branches can shade each other in competition of adatom supply from the diffusion field. This leads to ordering as branches coming too close will repel that way and those becoming too far will grow towards each other.

\subsubsection{Modeling pattern formation}

Insight into pattern formation can be gained from simplified models where ideas on microscopic mechanisms which underlie growth and drive shape transitions can be tested. In addition, such models provide a mathematical playground for the creation of fractal patterns that might later be discovered in nature. First modeling of snowflakes in fact dates back to the discovery and description of their fascinating properties. Kepler [284] attempted to explain the microscopic symmetry of snowflakes by examining the manner in which the elementary building units might pack together. Dalton [308] associated these building blocks with atoms and molecules. Dalton studied the straightforward problem as to how a two-dimensional flat surface can be covered with identical spheres. In the simplest hexagonal arrangement of close-packed spheres he was able to generate figures with the typical hexagonal symmetry of snowflakes. Equally he was able to construct "disordered" ramified patterns. 
The dendritic and the ramified patterns of Dalton are indeed the hallmark of the current approaches used to model pattern formation in growth processes. Modern-day approaches include the surface tension approach to model dendritic growth [289], a statistical mechanical approach based on Darcy's law to model tip splitting (observed in the fluid dynamics experiments described above) and dendritic growth [309,310], and the diffusion limited aggregation (DLA) model [15,16,311]. In the first approach dendritic patterns appear as the result of the competition between the non-isotropic diffusion field amplifying the growth at aggregate sites with the steepest gradient of the supply-atom concentration, and the surface tension favoring flat interfaces. In the second model Darcy's law, which is valid for a process where a fluid with low viscosity is forced under pressure to displace a fluid with much higher viscosity, is applied to atomic growth. From this model randomly ramified clusters are produced for isotropic and random growth, whereas tip splitting is observed for noise reduction (see below) and dendrites when anisotropy is introduced. Finally, the DLA computer model clusters are formed as randomly diffusing particles stick irreversibly to the perimeter of the growing aggregate (see Fig. 30, for a survey on DLA and fractal growth see Ref. [292]).

The classical numerical DLA simulations always produce ramified fractal islands, no matter whether they are carried out on a lattice or on a flat surface (off-lattice-DLA) [311,312]. In order to reproduce the relevant dendritic structures, several approaches have been followed to modify the simple hit-andstick DLA mechanism. Noise reduction [313,314] and curvature dependent sticking probabilities [13]

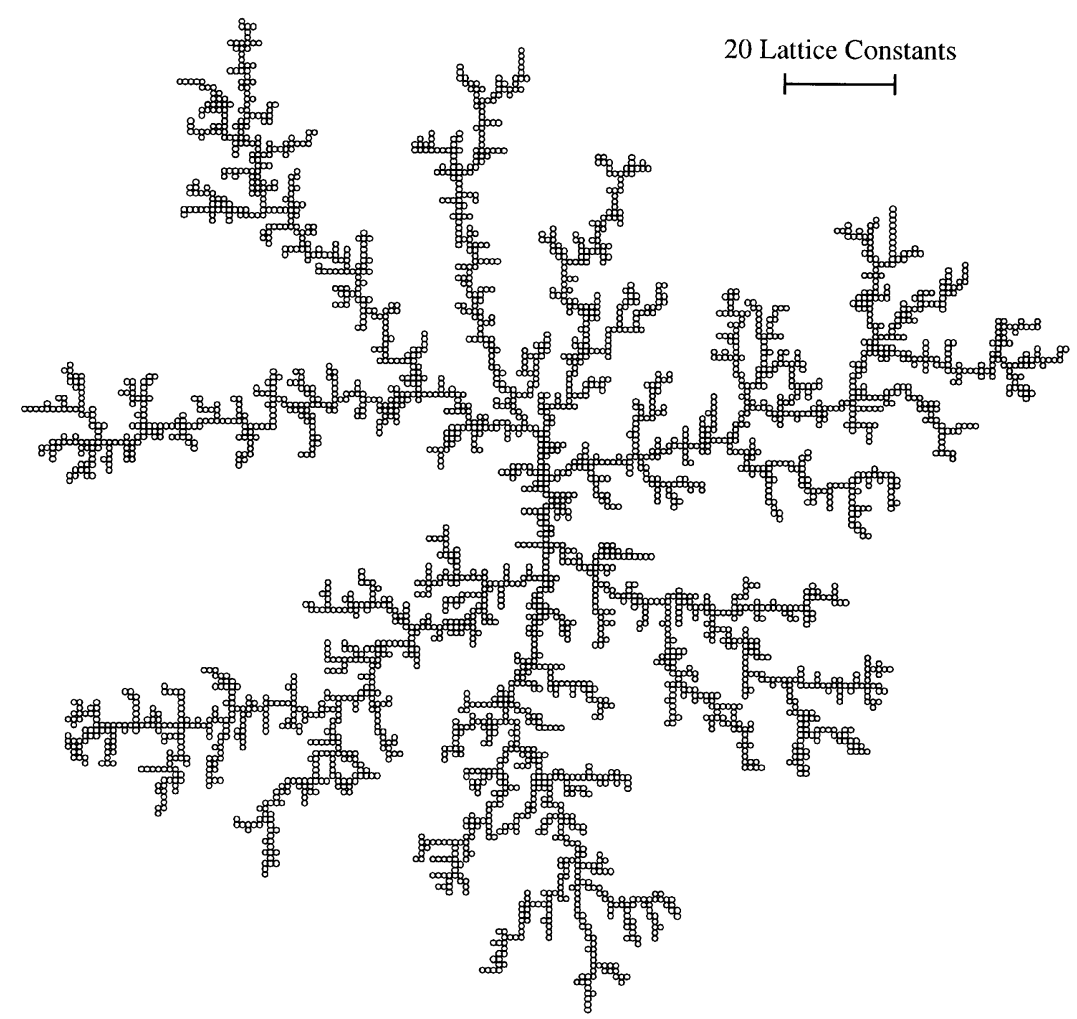

Fig. 30. Classical DLA aggregate of 3600 particles on a square lattice (from Ref. [15]). 
have been able to generate the desired transition from randomly ramified growth to oriented dendritic growth. In noise reduced DLA not all of the adatoms which hit the perimeter of the aggregate stick. A randomly walking adatom is assumed to stick to the perimeter only if the approached site has been contacted $m-1$ times before. For off-lattice-DLA simulations, there has been an approach called antenna mechanism which serves to introduce symmetry [315]. In this model the seed particle and each particle that subsequently attaches receives $n$ antennas pointing to the high symmetry directions, thus introducing the $n$-fold symmetry of a crystalline lattice. A particle arriving at an atom of the island border after its random walk over the structureless surface is rotated towards that antenna which is the closest to the contact sector and is attached there. This mechanism can be combined with noise reduction by giving each antenna a counter which is incremented when an atom hits the island in the vicinity of the antenna. Growth occurs only if a particle arrives in the contact sector belonging to an antenna with counter $m-1$, i.e. which has been contacted already $m-1$ times before. This "antenna" mechanism basically produces the same results as lattice DLA, i.e. for no noise reduction $(m=1)$ ramified fractals result whereas a transition to dendritic growth is observed with increasing noise reduction [312].

In general, the DLA models are quite successful as they incorporate the cross talk between different areas of the aggregate mediated by the diffusion field. In addition, the noise reduction approach can drive a transition from disordered patterns to dendrites [292,313]. With increasing noise reduction, lattice symmetry and thus anisotropy gains importance over randomness. However, two drawbacks of this approach are apparent. Both lie in the large amount to which the counter $m$ has to be increased in order to drive the transition. First, it is not clear why in experimental systems sticking should change over many orders of magnitude upon a slight variation of the growth conditions. Secondly, the clusters generated with this high noise reduction are highly symmetric and thus resemble needle crystals. Equally they lack statistical fluctuations which are to a certain extent characteristic for the dendrites.

\subsection{Dendritic growth at surfaces}

A common property of all systems that reveal a transition from random dominated to ordered symmetric patterns is anisotropy. Anisotropy competes with randomness which is intrinsic to nonequilibrium growth. Often the growth rate determines which one dominates. There are two central questions that immediately arise: what is the microscopic origin of anisotropy; and why is it manifested macroscopically only in a relatively narrow part of the parameter space [300]? For most of the shape transitions, such as in electrochemical deposition or crystal growth, these issues have not been answered satisfactorily. Recently, a shape transition from random to ordered interfacial patterns was reported for a highly ideal system, strictly 2D aggregation of $\mathrm{Ag}$ on a $\mathrm{Pt}\left(\begin{array}{lll}1 & 1 & 1\end{array}\right)$ single crystal surface [17]. As we will discuss in this section, the microscopic origin of anisotropy could be identified for this system and it was demonstrated how this microscopic detail is transferred to the overall pattern shape. In Section 5.3 we will address the question of what drives the transition to random growth.

\subsubsection{Crossover from random to dendritic growth}

A transition from random dominated to dendritic growth in non-equilibrium growth at surfaces was first observed for Ag aggregation on $\mathrm{Pt}\left(\begin{array}{lll}1 & 1 & 1\end{array}\right)$ [17]. The 2D Ag aggregates shown in Fig. 31(a) were grown at $110 \mathrm{~K}$ at a low $\mathrm{Ag}$ flux. Large clusters ( $~ 3000 \mathrm{Ag}$ atoms) with an open ramified structure are formed under these conditions. The branches of the clusters frequently alter their growth direction and 
a)

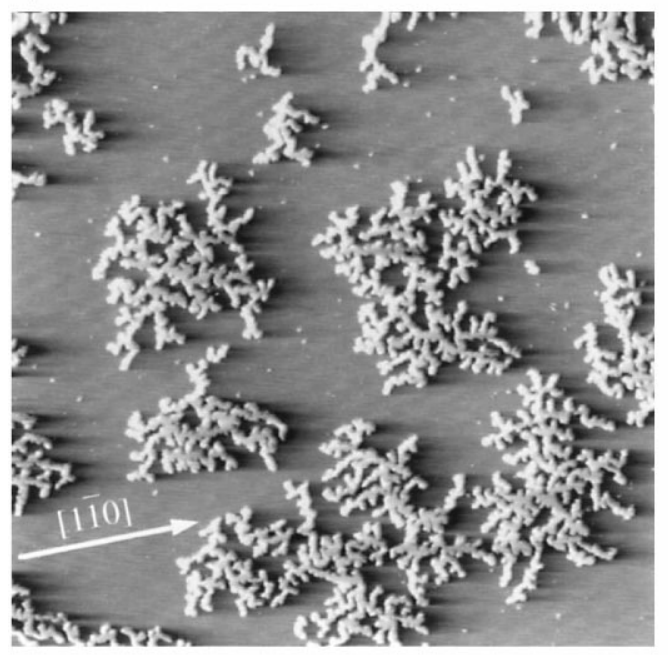

c)

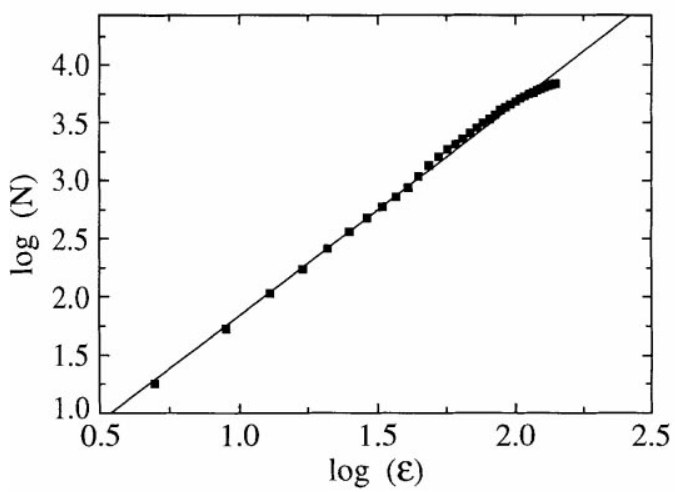

b)

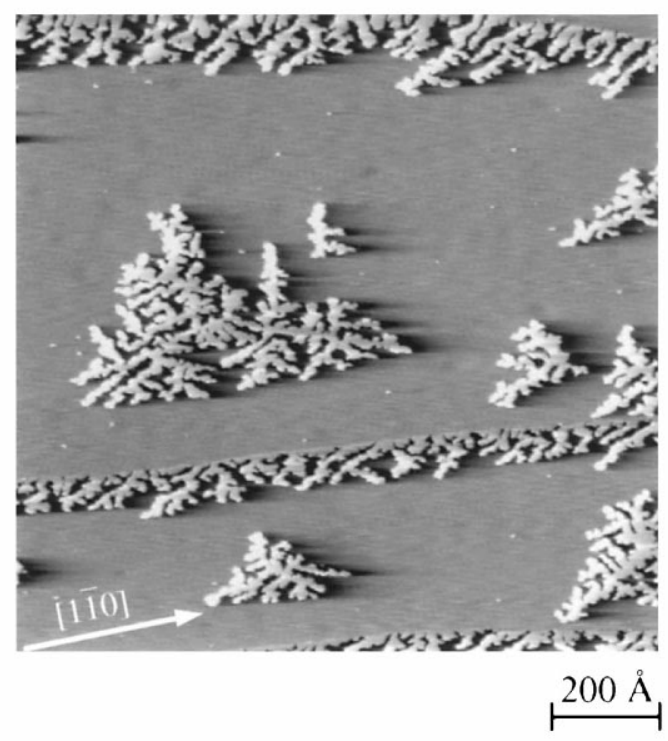

d)

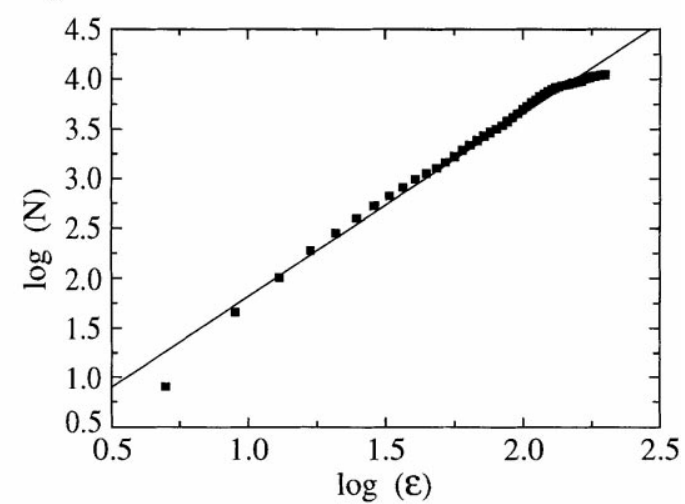

Fig. 31. Shape transition from random to dendritic fractal growth observed for Ag aggregation on a $\operatorname{Pt}\left(\begin{array}{lll}1 & 1 & 1\end{array}\right)$ surface with variable temperature STM. (a) Random aggregates grown at $110 \mathrm{~K}$ and a deposition flux of $1.6 \times 10^{-5} \mathrm{ML} / \mathrm{s}(\Theta=0.12 \mathrm{ML})$. (b) Dendritic patterns grown at $130 \mathrm{~K}$ with $F=1.1 \times 10^{-3} \mathrm{ML} / \mathrm{s}(\Theta=0.12 \mathrm{ML})$. (c) and (d) $\varepsilon$ is the border length of boxes in pixel units (1 pixel unit corresponds to $2.3 \AA^{2}$ ), $N$ is the number of pixels covered by Ag. These $\log N / \log \varepsilon$ plots allow fractal dimensions to be derived. The data in (c) correspond to the right bottom cluster in (a) and the fit gives a fractal dimension of $D=1.78$. The largest dendrite in (b) has a fractal dimension of $D=1.77 \pm 0.05$ (from Refs. $[17,281]$ ).

thus show no long range correlation with the trigonal substrate symmetry. The branches are of monoatomic height and are $2 \pm 1$ atoms wide. This width stays constant over the entire aggregate and is much smaller than its radius of gyration. The shape of the Ag aggregates grown at $110 \mathrm{~K}$ is very similar to that of the fractals simulated with the classical DLA computer codes, i.e. they are random dominated and therefore isotropic. In variance to the DLA cluster, however, the arm width is not monoatomic. 
A drastic change of the pattern shape was observed upon the use of a deposition rate a hundred times higher. Ag-clusters grown with this higher growth speed obey a nice dendritic pattern (see Fig. 31(b)). It has the characteristic back-bones, whose orientation is determined by the crystalline anisotropy of the substrate and the clusters very much resemble snowflakes (cf. Fig. 28(a)). In snowflake terminology this dendrite is of the P2a type (plane P, with irregular number of branches 2, three branched a). The trigonal symmetry of the dendrites arises from three preferential growth directions and leads to a trigonal envelope of the overall pattern.

The fractal character of each type of Ag aggregate was examined with the box counting method [14]. In both cases there is a regime of two orders of magnitude where the number of Ag-covered pixels $N$ scales with the box size $\varepsilon$ as $\varepsilon^{D}$ (see Figs. 31(c) and (d)). The fractal dimension obtained from masslength evaluation of numerous aggregates amounts to $D=1.76 \pm 0.07$ for the random aggregates, within error this is identical to the fractal dimension of $D=1.77 \pm 0.05$ found for the largest dendrite shown in Fig. 31(b). Both experimental values for $D$ are in good agreement with DLA simulations [15,16,311]. The scaling of the experimental structures is limited towards small lengths by the finite branch width of $2 \pm 1$ atoms (additional limitations arise from convolution with the STM-tip) and at large lengths by the finite cluster size. As mentioned in Section 5.1 the fractal dimension does not reflect the pattern symmetry.

The parameter that drives the crossover from ramified to dendritic patterns in the system $\mathrm{Ag} / \operatorname{Pt}\left(\begin{array}{lll}1 & 1 & 1\end{array}\right)$ is the deposition flux, i.e. the growth speed of the aggregate, which was changed by two orders of magnitude. The slight change in temperature has only negligible effect. Random patterns also form at $130 \mathrm{~K}$ through application of the low flux, and well-ordered dendrites evolve under the high flux at $110 \mathrm{~K}[128]$.

\subsubsection{Dendritic growth common to aggregation on hexagonal surfaces}

Let us first focus on dendritic growth. Dendritic growth with trigonal symmetry is commonly observed for low temperature aggregation with moderate deposition flux on hexagonal close-packed surfaces. The STM images reproduced in Fig. 32 show examples for a variety of metal systems. The patterns have the common feature that their branches preferentially grow in three directions which are rotated by $120^{\circ}$ with respect to each other. These directions of branch growth are the substrate $\langle\overline{1} \overline{1} 2\rangle$ directions, which are perpendicular to so-called A-steps. One of these directions was labeled A in Fig. 32 for each system where the crystallographic orientation was identified. For $\mathrm{Ag} / \mathrm{Ag}(111)$ (Fig. 32(a)) the preferred growth in three directions is clearly seen from the central branches of the aggregates. The trigonal symmetry of the aggregates on one pseudomorphic Ag layer is even more pronounced (Fig. 32(b)). Their shape resembles very much that of needle crystals, which are the extreme case of anisotropic growth [313]. The example for $\operatorname{Pt}(111)$ homoepitaxy shows a greater degree of randomness, consequently it was first interpreted as the physical realization of DLA [279]. Recently, it was recognized as being dendritic growth revealing the same growth directions as found in $\operatorname{Ag} / \operatorname{Pt}\left(\begin{array}{lll}1 & 1 & 1\end{array}\right)$ [282]. The recent examples for $\mathrm{Au} / \mathrm{Pd}\left(\begin{array}{lll}1 & 1 & 1\end{array}\right)$ [316] and Pt/Ru(0 0001$)$ (see Fig. 32(d) ${ }^{48}$ ) also show the trigonal symmetry characterizing dendrites. Note that in the latter case the substrate is not trigonal. As a consequence of the hcp stacking the dendrites rotate by $60^{\circ}$ from terrace to terrace. These examples strongly suggest that dendritic growth is universal for low temperature metal aggregation on hexagonal close-packed metal surfaces at normal growth rates.

\footnotetext{
${ }^{48}$ Fig. 32(d) was kindly provided by B. Gleich, M. Scherrer, A. Hitzke, and R.J. Behm prior to publication.
} 
a)
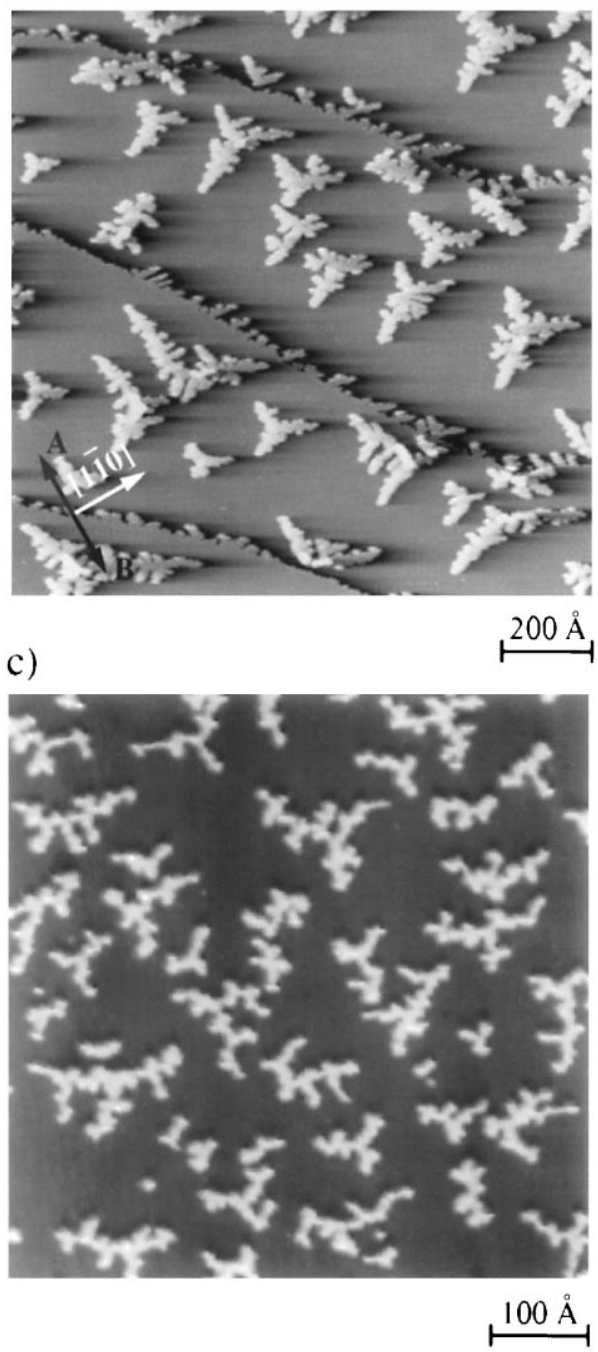

b)
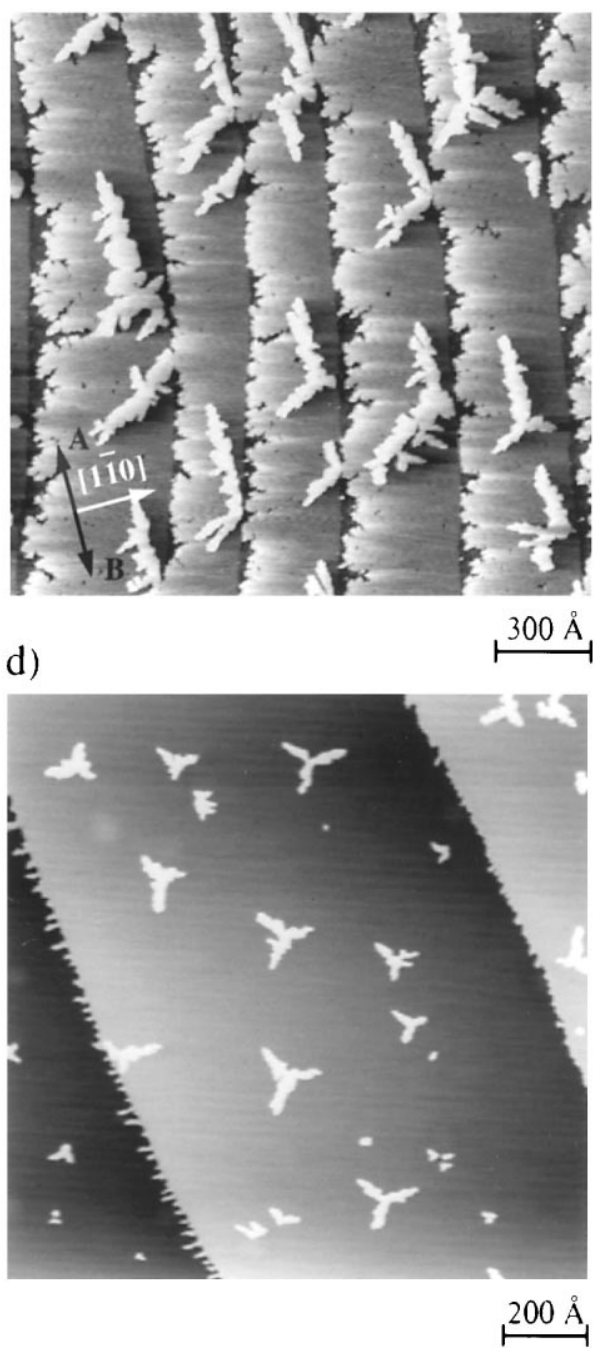

Fig. 32. Dendritic patterns formed for low temperature metal deposition onto hexagonal close-packed substrates. (a) Ag on $\mathrm{Ag}(111)$ at $110 \mathrm{~K}$ and (b) $\mathrm{Ag}$ adsorbed at $170 \mathrm{~K}$ on the pseudomorphic first $\mathrm{Ag}$ layer on $\mathrm{Pt}(1111)$ [280,281]. (c) Dendrites in $\mathrm{Pt}(111)$ homoepitaxy at $180 \mathrm{~K}$ [282]. (d) Dendritic growth observed for Pt on $\mathrm{Ru}(0001)$ at $300 \mathrm{~K}$. (Coverages: (a) and (b) $0.12 \mathrm{ML}$, (c) $0.094 \mathrm{ML}$, and (d) 0.1 ML.)

In order to analyze the microscopic origin of dendrite formation on hexagonal surfaces, we distinguish two diffusion processes that can take place at the island edge. Depending on whether an adatom starts from a site which is laterally two- or one-fold coordinated to the island, this displacement is called edge- or corner-diffusion, respectively. ${ }^{49}$ This definition does not include the final coordination. It is reasonable to call a process edge-diffusion only in the case where the final coordination is also

${ }^{49}$ This important difference was first realized by Zhang et al. [88]. 
two-fold. The case where an edge atom goes to a corner opens up corner crossing. This process marks the transition to compact islands; it becomes important at higher temperatures and should be treated separately.

It turns out that the distinction between corner- and edge-diffusion is rather useful as these processes have a quite different effect on the aggregate's shape. Edge diffusion leads to a thickening of the aggregate's branches [92,317-319]. Closer inspection of Fig. 32 reveals that the dendrites in the various systems have quite different branch widths, ranging from $18 \pm 3$ atoms for the cluster grown on $1 \mathrm{ML}$ $\mathrm{Ag} / \mathrm{Pt}(111)$ at $170 \mathrm{~K}$ [280] to 2-3 atoms for the dendrites formed at $180 \mathrm{~K}$ in $\mathrm{Pt}(111)$ homoepitaxy [282]. This indicates a different amount of edge-diffusion involved in the pattern formation. However, the trigonal symmetry is independent of the branch width, ruling out edge diffusion as its origin. On the other hand, the smallest branch widths of 2-3 atoms, as well as the anisotropic cluster shape, rule out a sample hit-and-stick mechanism since this would yield monoatomic branches and isotropic patterns $[17,282]$.

\subsubsection{Initial branching}

The first indication for the mechanism underlying dendritic growth came from looking at the initial branching of the cluster seed for the system $\mathrm{Ag} / \mathrm{Pt}(111)$ [17]. At $80 \mathrm{~K}$ small $\mathrm{Ag}$ islands form, their mean size being controlled by coverage. The smallest islands in Fig. 33(a) appear almost spherical and comprise about seven atoms suggesting the compact seed particle to be a heptamer (this size is not important for the suggested mechanism to be operative). As larger islands form they take on a Y-shape with arms always subtending angles of $120^{\circ}$. Fig. 33(b) shows larger clusters, all of them branched into Y-form. Most remarkably, all these Y's are oriented the same way. Y-branching of a heptamer is possible by atom attachment to so-called A- or B-steps (see left- and right-hand side, respectively, in the model displayed in Fig. 33(c)). These two types of steps generally characterize hexagonal close-packed surfaces. Whenever an island is put onto such a surface, it is bound by a close-packed $\{111\}$-facet towards one direction, whereas the step pointing in the opposite direction is a more open $\{100\}$ facet. $^{50}$ These two micro-facets formed by $\langle 1 \overline{1} 0\rangle$-oriented steps were labeled B- and A-steps, respectively [320].

A- and B-steps can be distinguished by their different surface free energies. This becomes apparent through the thermodynamic equilibrium shape of vacancy islands [321]. In order to identify the preferred directions into which branching occurs, Ag was deposited at $100 \mathrm{~K}$ onto a $\operatorname{Pt}(111)$ surface purposely prepared with vacancy islands to act as a template [281]. From inspection of Fig. 33(d) it is seen that branches inside the quasi-hexagonal vacancies grow perpendicular to the shorter A-steps, whereas they form an angle of $30^{\circ}$ with the longer B-steps (identical observations were made at both types of steps for $\mathrm{Pt} / \mathrm{Pt}\left(\begin{array}{lll}1 & 1 & 1\end{array}\right)$ [282]). Similarly the Y's that form in-between the vacancies have their branches exclusively oriented perpendicular to the three A-steps, representing the crystallographic $\langle\overline{1} 12\rangle$-directions. Preferred branching into these directions leads to the trigonal symmetry of the Y's, which can be considered as the smallest dendrites. The larger dendrites reveal their longest branches exactly in the same directions (see for example Fig. 30(b)). The examples from other systems reported in Fig. 32, commonly reveal preferred branching into the three $\langle\overline{1} \overline{1} 2\rangle$-directions (for those cases where the crystallographic orientation was identified).

\footnotetext{
${ }^{50}$ This labeling is valid for fcc(1 111$)$ surfaces, the same step symmetry also characterizes the $\left(\begin{array}{lll}0 & 0 & 1\end{array}\right)$ surface of a hexagonal crystal.
} 
a)

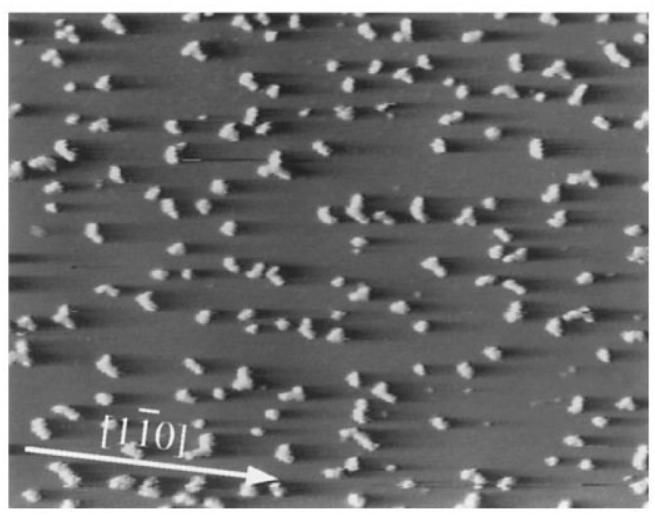

c)

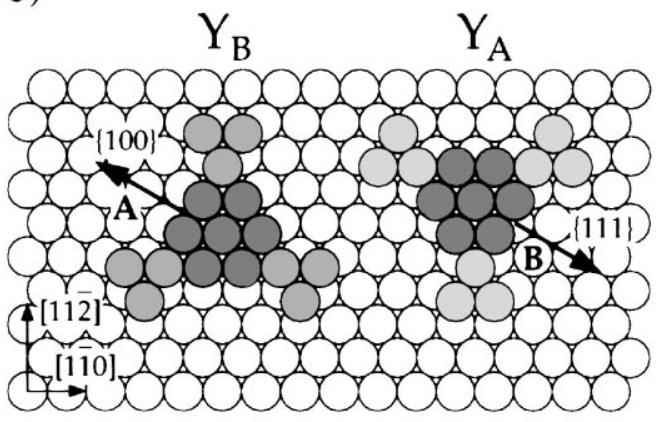

b)

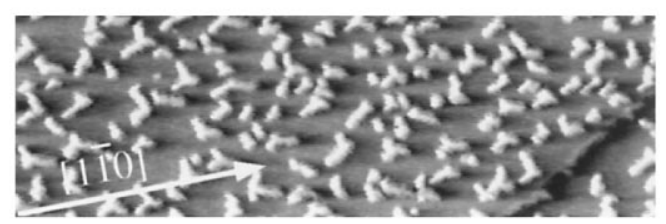

d)

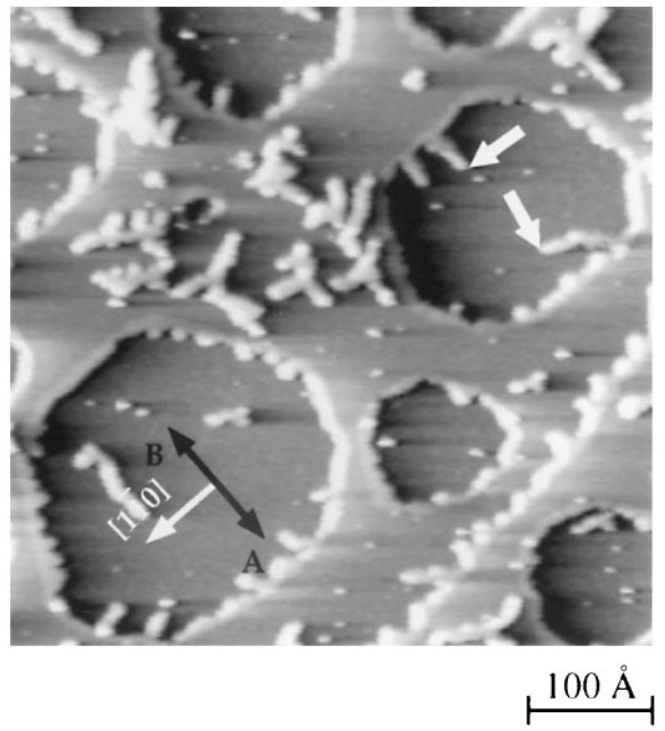

Fig. 33. Tracing back the mechanism for dendritic growth for $\mathrm{Ag} / \mathrm{Pt}(111)$ ). (a) and (b) STM images of $\operatorname{Ag}_{n}$ clusters grown on $\operatorname{Pt}\left(\begin{array}{lll}1 & 1\end{array}\right)$ at $80 \mathrm{~K}$ with an average size of $n=13$ in (a) and $n=22$ atoms in (b). Branching sets in for particle sizes exceeding about seven atoms. Preferred branching into three directions leads to Y-forms that are oriented the same way. (c) Two possible orientations of the Y-cluster (from Ref. [17]). (d) Identification of the growth directions. A Pt(1 1 1 1$)$ substrate with vacancy templates was prepared through removal of $0.5 \mathrm{ML}$ by sputtering at $600 \mathrm{~K}$ and subsequent annealing to $700 \mathrm{~K}$. Then 0.12 ML Ag were adsorbed at $100 \mathrm{~K}$. Preferred growth is perpendicular to A-steps. Material attached to B-steps of the substrate develops tip instability perpendicular to A-steps (see arrows) (from Ref. [281]).

The observed branching perpendicular to A-steps involves preferred attachment towards this step type. Accordingly, DLA simulations, where growth is only allowed for particles attaching from three of the possible six directions on a hexagonal lattice, procedure patterns resembling the trigonal dendrites seen in experiment [292]. These simulations do not furnish information on the mechanism responsible for this selection rule. Nevertheless, they show that anisotropy is important for dendritic growth. This can equally be inferred from experiments that were designed to demonstrate the role of ansiotropy, for example in the Saffman-Taylor instability [305-307].

\subsubsection{The mechanism for dendritic growth}

The mechanism leading to preferred population of A-steps on close-packed metal surfaces arises because of two asymmetries associated with the geometric differences of A- and B-steps. The first is the anisotropy of diffusion from corner sites towards the two types of steps, and was discovered 
a)

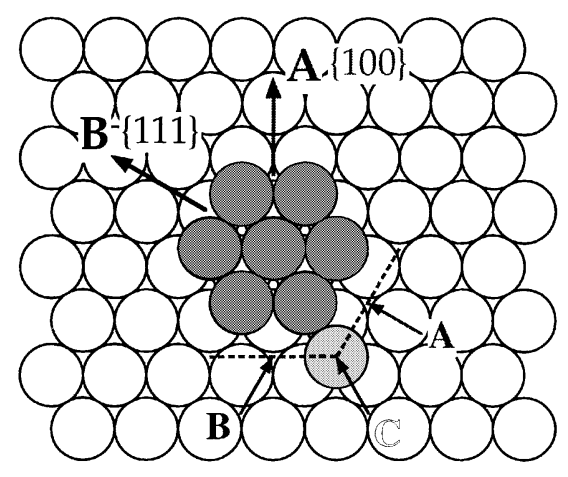

b)

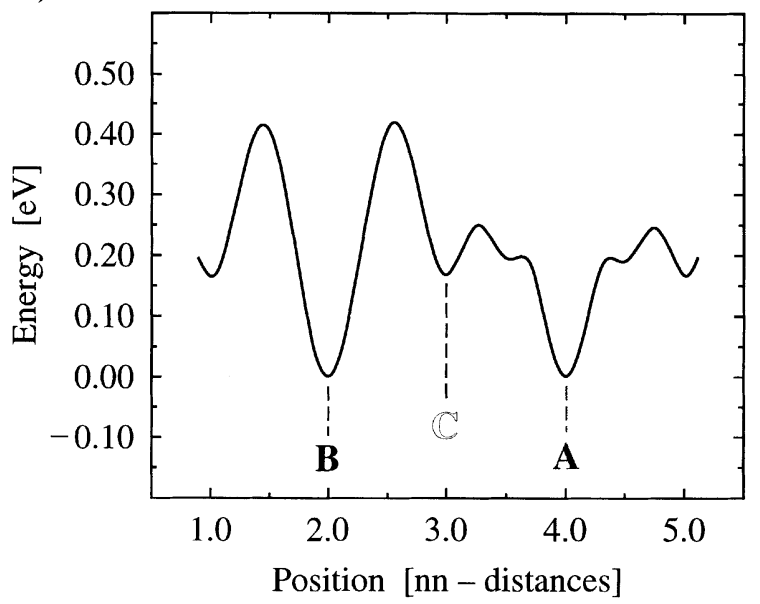

Fig. 34. EMT calculations showing the difference in total energy of a $\mathrm{Ag}$ adatom diffusing around a $\mathrm{Ag}$ heptamer on $\mathrm{Pt}\left(\begin{array}{lll}1 & 1 & 1\end{array}\right)$ (A- and B-steps are indicated in the ball model). The diffusion process with the lowest barrier is from a corner site (C) to an A-step (from Ref. [280]).

independently by two research teams [280,282]. The second is the asymmetry in the diffusion path towards the two steps [280]. While the first is system specific, the second is largely general as it depends less on misfit and edge relaxation.

Let us first focus on corner diffusion. The EMT results for $\mathrm{Ag} / \mathrm{Pt}\left(\begin{array}{lll}1 & 1 & 1\end{array}\right)$ displayed in Fig. 34(b) show that diffusion of a corner bound atom (c) to an A-step has a much smaller barrier than its displacement towards a B-step. This asymmetry can be rationalized using simple geometric arguments. From inspection of Fig. 34(a) it becomes apparent that displacement from a corner to an A-step can be done via an hep-hollow site without losing the lateral coordination to the heptamer throughout this motion. ${ }^{51}$ In contrast, for diffusion towards a B-step the hcp-site is located too close towards the island, thus the adatom has to walk over an on-top site, which is much more costly in energy. This asymmetry also expresses itself in two types of dimer motion, an intracell rotation and an intercell translation. The first involves the transition state also involved in corner to A-step diffusion, while the second requires passing by the costly transition state of corner to B-step displacement (see discussion of dimer mobility in Section 3.2).

The growth scenario (Fig. 35(a)) shows how preferred diffusion from corner sites towards A-steps determines the shape of the growing aggregate [280]. For symmetry reasons we start from a tetramer bounded by two A- and B-steps. Such a tetramer has three corners adjacent to an A-step and atoms arriving there end up at A-steps through corner diffusion (see atoms 1 and 2). Therefore A-steps disappear due to their preferred population and the tetramer transforms into a triangle exclusively bounded by B-steps. ${ }^{52}$ The three resulting corners are exposed to a larger angle of the diffusion field and therefore have a slightly higher capture rate than straight steps (the higher growth rates of

\footnotetext{
${ }^{51}$ The total energy displays a shallow local minimum at that hep-site, see Fig. 34(b).

${ }^{52}$ This exactly follows the basic rule of crystal growth, where rapidly growing facets disappear and the ones with smallest growth speed determine the final crystal shape.
} 
a)

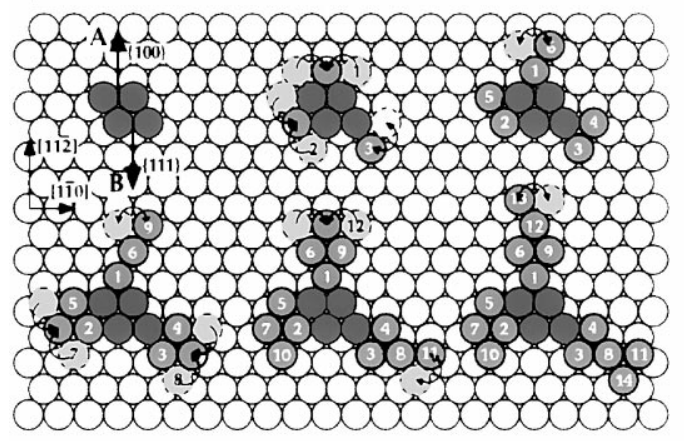

b)

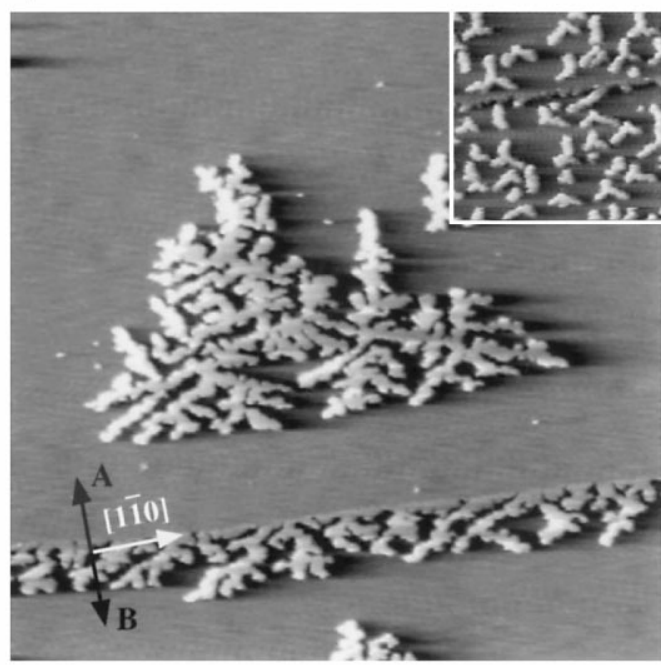

c)

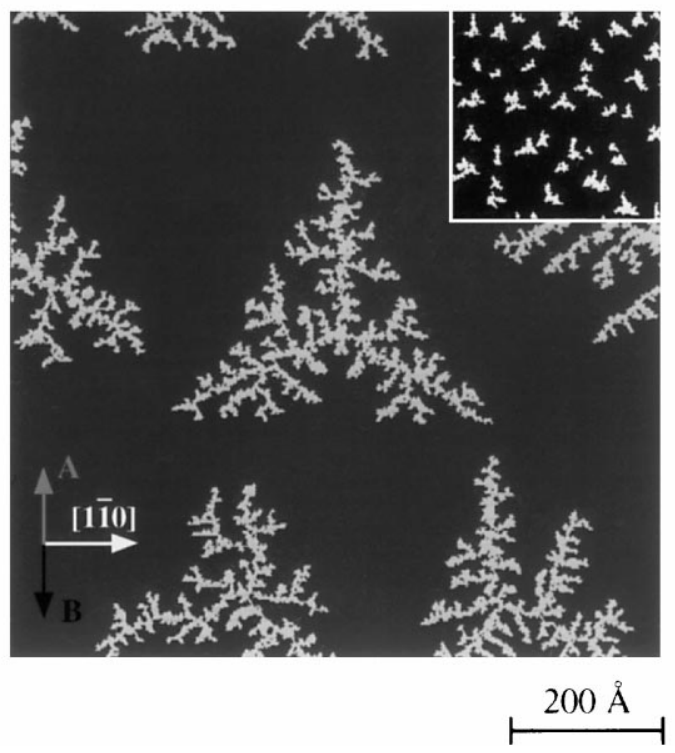

Fig. 35. Verification of the mechanism leading to dendritic growth. (a) Growth scenario showing how dendritic Y's with branches pointing into the A-directions grow on a hexagonal surface if corner diffusion towards A-steps is active while that towards B-steps is frozen. See also relaxation of a dimer pointing away from the heptamer towards a dimer orientation providing higher coordination, cf. atoms 6 and 9. (b) STM images showing dendrites for $\mathrm{Ag} / \mathrm{Pt}\left(\begin{array}{lll}1 & 1 & 1\end{array}\right)$ at $130 \mathrm{~K}$ (main figure) and $80 \mathrm{~K}$ (inset) in comparison with results from KMC simulations. (c) The parameters used in the simulations are $E_{\mathrm{m}}=160 \mathrm{meV}, E_{\mathrm{C} \rightarrow \mathrm{A}}=160 \mathrm{meV}, E_{\mathrm{C} \rightarrow \mathrm{B}}=320 \mathrm{meV}$, relaxation of dimer-chain $E_{\mathrm{Ex}}=160 \mathrm{meV}$ (in the simulation at $130 \mathrm{~K}$ $E_{\mathrm{m}}$ was reduced to $120 \mathrm{meV}$ to account for the lower island density through $i=2$ ) (from Ref. [280]). 
protrusions is known as "tip effect" [16] or Mullins-Sekerka instability [322]). Each atom attaching to such a corner becomes trapped (see atom 3). It rapidly diffuses back and forth between the two corner sites, but it cannot escape from there, since this would be diffusion to the adjacent B-steps which is assumed to be frozen. Such an atom by itself forms a protruding tip creating a site with high capture probability for a second atom to form a dimer providing a new A-step. The second atom can either arrive at the respective free corner, or at a site more apart from the island forming a dimer with its axis pointing to the terrace. The first case leads to a new A-step with a high collection probability and growth in the A-direction proceeds. In the second case, as suggested from EMT calculations for $\mathrm{Ag} / \mathrm{Pt}\left(\begin{array}{lll}1 & 1 & 1\end{array}\right)$, the dimer can relax back to the more compact form of the first case, thus also yielding an A-step [280]. By this growth proceeds in the A-direction and the scenario successfully explains the formation of Y's.

Ideas on microscopic mechanisms are best tested with KMC simulations. Such simulations were performed for $\mathrm{Pt} / \mathrm{Pt}\left(\begin{array}{lll}1 & 1 & 1\end{array}\right)$ [282] and $\mathrm{Ag} / \mathrm{Pt}\left(\begin{array}{lll}1 & 1 & 1\end{array}\right)$ [280]. They demonstrate how preferred corner diffusion towards A-steps leads to dendrites with the experimentally observed symmetry and orientation. A thorough test of a model is whether it is able to describe the experiment at different temperatures with a single set of parameters. Convincing agreement of the KMC simulatons with the STM images for 130 and $80 \mathrm{~K}$ was achieved for $\mathrm{Ag} / \mathrm{Pt}\left(\begin{array}{lll}1 & 1\end{array}\right)$ (compare Figs. 35(c) and (b)). It should be noted that $\mathrm{Pt} / \mathrm{Pt}\left(\begin{array}{lll}1 & 1 & 1\end{array}\right)$ displays much more randomness than $\mathrm{Ag} / \mathrm{Pt}$. Accordingly the anisotropy of the two corner processes was weaker in the simulations that were designed for $\mathrm{Pt} / \mathrm{Pt}\left(\begin{array}{lll}1 & 1 & 1\end{array}\right)$ (compare activation energies $E_{\mathrm{C} \rightarrow \mathrm{A}} / E_{\mathrm{C} \rightarrow \mathrm{B}}=330 \mathrm{meV} / 500 \mathrm{meV}$ for $\mathrm{Pt} / \mathrm{Pt}(111)$ vs. $160 \mathrm{meV} / 320 \mathrm{meV}$ for $\mathrm{Ag} / \operatorname{Pt}\left(\begin{array}{lll}1 & 1 & 1\end{array}\right)$ ). The dimer instability at a corner (see atoms 6 and 9) had to be included for $\mathrm{Ag} / \mathrm{Pt}\left(\begin{array}{lll}1 & 1 & 1\end{array}\right)$ to satisfactorily reproduce its pronounced anisotropy [280]. Independent from such details, one of the basic atomic mechanisms underlying dendritic growth is anisotropy in diffusion from corner sites towards the two types of steps generally present at surfaces with hexagonal symmetry.

\subsubsection{General implications}

So far, it has been demonstrated that preferential diffusion from corner sites towards A-steps is responsible for dendritic growth for the systems $\mathrm{Ag} / \mathrm{Pt}\left(\begin{array}{lll}1 & 1 & 1\end{array}\right)$ [280] and $\mathrm{Pt} / \mathrm{Pt}\left(\begin{array}{lll}1 & 1 & 1\end{array}\right)$ [282]. For $\mathrm{Ag} / \mathrm{Pt}\left(\begin{array}{lll}1 & 1 & 1\end{array}\right)$ the argument was partly based upon EMT calculations. These calculations show the same corner-diffusion asymmetry for two other systems where dendritic growth was observed, namely $\mathrm{Ag} / \mathrm{Ag}\left(\begin{array}{lll}1 & 1 & 1\end{array}\right)$, and $\mathrm{Ag} / 1 \mathrm{MLAg} / \operatorname{Pt}\left(\begin{array}{lll}1 & 1 & 1\end{array}\right)$ [280]. For $\mathrm{Pt} / \mathrm{Pt}\left(\begin{array}{lll}1 & 1 & 1\end{array}\right)$, however, the calculations predict a (weak) preference for corner to B-step diffusion (see Fig. 36(b) and Refs. [134,280]). The quantitative comparison between KMC simulations and experiment (in terms of the in-plane coordination) for $\mathrm{Pt} / \mathrm{Pt}\left(\begin{array}{lll}1 & 1 & 1\end{array}\right)$ shows in contrast to the prediction from EMT that diffusion from corners to B-steps becomes activated well after that to A-steps [282]. The EMT calculations for $\operatorname{Pt} / \operatorname{Pt}(111)$ may, however, not be entirely wrong. It is very reasonable that the asymmetry for the corner process is in fact less pronounced for Pt homoepitaxy. Pt reveals a large tensile stress leading to a strong inward contraction at the island edge. This can facilitate diffusion towards B-steps as it renders the hcp-site more attractive as a transition state, which otherwise appears too close to the island (Fig. 34(a)). Due to this geometric argument, one would expect diffusion towards B-steps equally to be easier for adsorbates with a significant negative misfit. This is corroborated by the EMT results for $\mathrm{Cu} / \mathrm{Pt}\left(\begin{array}{lll}1 & 1 & 1\end{array}\right)$ represented in Fig. 36(b). The fact that the EMT calculations seem to overestimate the effect of tensile stress, however, renders general predictions on which systems might reveal dendritic growth, and into which direction, solely based on such calculations uncertain. 
a)

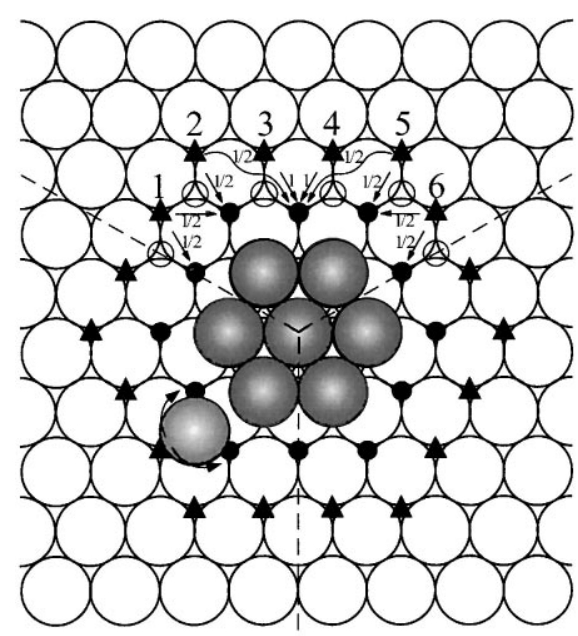

b)

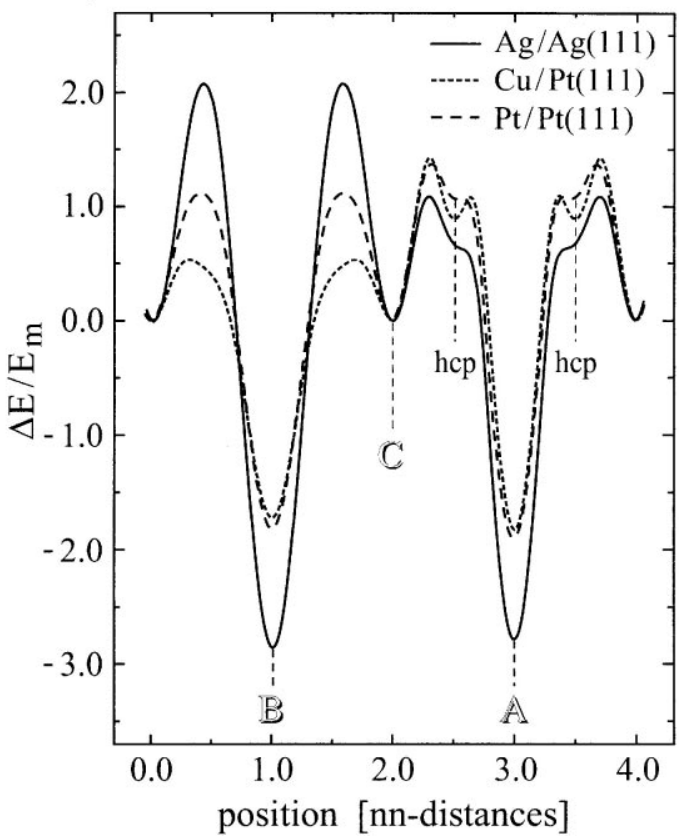

Fig. 36. Preferred attachment towards A-steps is intrinsic for all surfaces with hexagonal symmetry. (a) Model showing how atoms from nearest neighbor sites are funneled preferentially towards A-steps. This asymmetry is caused by hcp-sites located in phase with the step atoms for A-steps, whereas they are out-of-phase and also far apart at B-steps. (b) EMT calculations for diffusion around a heptamer as in Fig. 34(a), for a number of systems. They all show the asymmetry around the hcp site located before an A-step whereas corner diffusion is system specific. The energy barriers were scaled by the EMT-values for terrace diffusion of the respective system.

Up to now, we have focused on the diffusion of atoms after attachment to an island. Let us now turn to the role of the diffusion paths towards these two kinds of steps [280]. The different ways an atom can attach itself to a heptamer are displayed in Fig. 36(a). Consider diffusion starting from the fcc-sites located one atomic distance apart from the heptamer (marked by triangles), onto sites adjacent to the heptamer (marked by filled circles). For symmetry reasons it suffices to regard one of the $120^{\circ}$ segments indicated. Diffusion towards the island will occur via one of the hep-sites situated immediately after our initial site (these hcp-sites are marked by open circles). There are two differences between the two types of steps regarding these hcp-sites. Firstly, the A-step has two such hcp-sites whereas the B-step has three. Those associated with the A-step lie closer to the step than those associated with the B-step. Second, the hcp-sites at an A-step are in line with respect to the step atoms (when looking perpendicularly to the step) whereas those before the B-step are out of phase.

The proximity of the hcp-sites to the A-step implies that an atom located there is subject to strong interaction with the island. This interaction is asymmetric due to the different lateral coordinations attained at the two final fcc-sites (see atom shaded in lighter gray in Fig. 36(a)). Consequently, the barrier for diffusion to the two-fold A-step is much smaller than for diffusion to a one-fold corner. This asymmetry appears to be common to all systems, independent of the details on edge relaxation (see EMT results in Fig. 36(b) for various systems and also Fig. 34(b) for $\mathrm{Ag} / \mathrm{Pt}\left(\begin{array}{lll}1 & 1 & 1\end{array}\right)$ ). Atoms diffusing to 
B-steps, on the other hand, chose one of the hcp-sites at random without feeling much of the island. Subsequently, they diffuse straight towards their final attachment site which can be a corner or an edge site with equal probability. Accordingly, the atoms starting from sites 1 and 6 attach with equal probabilities to B-steps and corners. Atoms starting from sites 3 and 4 end up at the A-step, and those coming from sites 2 and 5 go to a corner or, with equal probability, to the A-step (via sites 3 and 4). This yields a strong statistical preference of $\frac{3}{6}$ vs. $\frac{1}{6}$ for attachment to A- vs. B-steps, corners are visited with a probability of only $\frac{2}{6}$.

This general asymmetry in attachment is an additional consideration to the effect of corner diffusion. If corner diffusion is faster towards B-steps, it could counterbalance attachment and random growth may result. For systems with faster diffusion towards A-steps, both effects push towards exclusive

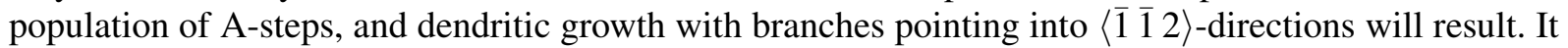
will be very interesting to look at systems with large negative misfit, where corner diffusion is expected to be faster towards B-steps, and check whether these exhibit random growth. Current KMC models do not contain diffusion via hcp sites. This needs to be incorporated to account for the attachment asymmetry between the two types of steps characterizing hexagonal substrates.

\subsubsection{No classical DLA clusters for metal aggregation}

These recent findings on attachment to islands and diffusion from corner sites on hexagonal lattices have important general implications for aggregation at low temperatures. Both relaxation processes are expected to have barriers that are smaller or within the range of the barriers for terrace diffusion (see scaled EMT results in Fig. 36(b)). This implies that they become activated as soon as nucleation and aggregation set in. Therefore the classical hit-and-stick DLA clusters very likely never form at

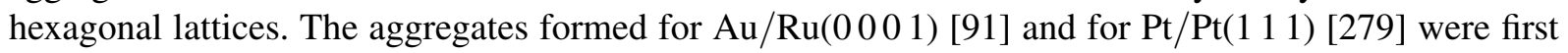
considered as physical realization of DLA. However, it was later realized that neither pattern represents

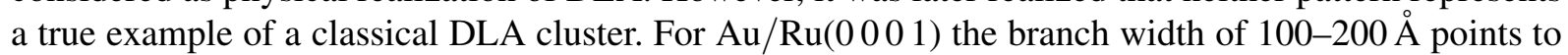
a considerable amount of edge diffusion [92,317-319]. For $\mathrm{Pt} / \mathrm{Pt}\left(\begin{array}{ll}1 & 1\end{array}\right)$ the islands became identified as dendrites as discussed above. There are several experimental observations supporting the conclusion that DLA-growth cannot be achieved for metal epitaxy on hexagonal substrates. For $\operatorname{Ag} / \operatorname{Pt}\left(\begin{array}{lll}1 & 1 & 1\end{array}\right) \mathrm{Y}$ branching was reported down to the lowest temperature $(60 \mathrm{~K})$ at which islands can reach the critical size for branching before coalescence [128]. Similar observations were made for $\operatorname{Ag} / \operatorname{Ag}(111)$. For $\mathrm{Pt} / \mathrm{Pt}\left(\begin{array}{lll}1 & 1 & 1\end{array}\right)$ diffusion towards A-steps starts at $130 \mathrm{~K}$ [282]. This is close to the onset temperature of terrace diffusion of $110 \mathrm{~K}$ [37]. The required diffusion rate for edge relaxation processes to become effective is generally determined by the lateral impingement rate $I=F n_{x}^{-1}=\eta^{-1 / 3} F^{2 / 3} D^{1 / 3}$ (upon application of Eq. (8) of Section 2 for $i=1$ ). For reasonable fluxes this rate is always much slower than that of terrace diffusion, making the existence of a DLA regime between 110 and $130 \mathrm{~K}$ for $\mathrm{Pt} / \mathrm{Pt}\left(\begin{array}{lll}1 & 1 & 1\end{array}\right)$ very unlikely.

These experimental observations contradict theoretical papers which suggest the existence of an extended DLA-regime for hexagonal lattices [88,121,323]. However, these theoretical studies disregard important characteristics of aggregation on hexagonal surfaces. Although the difference of corner and edge diffusion was correctly pointed out in the first study [88], there was no distinction in diffusion towards the two types of steps. In particular that these processes might have barriers as low as that of terrace diffusion was not recognized [88]. In KMC simulations based on detailed ab initio results for $\mathrm{Al} / \mathrm{Al}\left(\begin{array}{lll}1 & 1 & 1\end{array}\right)$ [121,323] the authors did not calculate the barriers for corner diffusion. They ascribed corner diffusion the same barrier as edge diffusion and consequently obtained DLA-growth at $50 \mathrm{~K}$. 
The present experimental knowledge strongly suggests that classical monoatomic branched DLA clusters are very unlikely on hexagonal surfaces. On square lattices edge atoms are only one-fold coordinated. Consequently edge diffusion was often reported to have a small barrier comparable to, or even below that of terrace diffusion $[114,121,178]$. Therefore DLA clusters are equally unexpected for square lattices. This is in line with the observation of exclusively compact islands on these lattices $[79,86,324-326]$. The only exception to this rule was observed for $\mathrm{Cu} / \mathrm{Ni}(100)$ where the strain energy is thought to drive non-compact island shapes. However, also these are not classical DLA clusters as they have arms being $\sim 22$ atoms wide [90].

\subsection{Shape transitions at surfaces}

Section 5.2 showed that growth of dendrites with trigonal symmetry is well understood and that this growth morphology is expected to be the rule for low temperature aggregation on hexagonal metal surfaces. Dendritic patterns undergo several mutations upon increasing the temperature and/or reducing the flux. For some systems the branches begin to grow in random directions lifting the trigonal symmetry of the low temperature dendrites and in addition the branches become wider. Despite a considerable effort, the shape transition from dendritic to random fractals for $\operatorname{Ag} / \operatorname{Pt}(111)$, as well as the random isotropic growth patterns often observed at higher temperatures [91,92,263], are not yet fully understood. We will discuss preliminary results that can explain the transition for $\operatorname{Ag} / \operatorname{Pt}\left(\begin{array}{lll}1 & 1 & 1\end{array}\right)$. We also review studies relating the degree of edge diffusion to the branch width. Also here, full quantitative understanding is presently missing. Compact islands, sometimes with triangular shape, follow the random fractals with increase in temperature. For $\operatorname{Pt} / \operatorname{Pt}\left(\begin{array}{lll}1 & 1 & 1\end{array}\right)$ random fractals were not observed and the shape transition proceeds directly from dendrites to manifold compact island shapes [279]. The transition from ramifield to compact islands has attracted considerable attention [317-319], and several very different models have been proposed to explain the various compact island shapes observed for $\mathrm{Pt} / \mathrm{Pt}(111)$ [279,327,328]. In this section we review the present understanding of island shape transitions on hexagonal surfaces.

\subsubsection{Attempts to understand random fractal patterns}

A transition from dendrites towards random fractal patterns was observed for $\operatorname{Ag} / \operatorname{Pt}\left(\begin{array}{lll}1 & 1 & 1\end{array}\right)$ upon lowering the deposition flux. Two STM images for growth at $110 \mathrm{~K}$ are shown in Fig. 37 in comparison with KMC simulations. The patterns generated at moderate deposition flux ( $1 \mathrm{ML} / 900 \mathrm{~s}$ ) clearly show preferred growth directions, evident through their triangular envelope and the parallel branches growing out from the substrate steps (Fig. 37(a)). This trigonal symmetry is entirely lifted when the growth speed is lowered by two orders of magnitude. In the STM image in Fig. 37(b) the branches often change their growth directions resulting in random dominated fractals. The frequent change in growth direction in the fractal patterns may be the key to their understanding. For the dendrites to form it is essential that growth exclusively takes place on fcc-sites. If for some reason, hcp-site domains started to form, the Aand B-directions would become exactly inverted with respect to fcc-domains (see Fig. 37(e)). Frequent changes in stacking would turn the preferred growth directions back and forth by $60^{\circ}$ and produce random isotropic patterns. The simulations in Figs. 37(c) and (d) demonstrate that creation of dislocations can indeed drive a transition from dendritic to random fractal growth, and that this happens solely upon variation of the deposition flux. 
a)

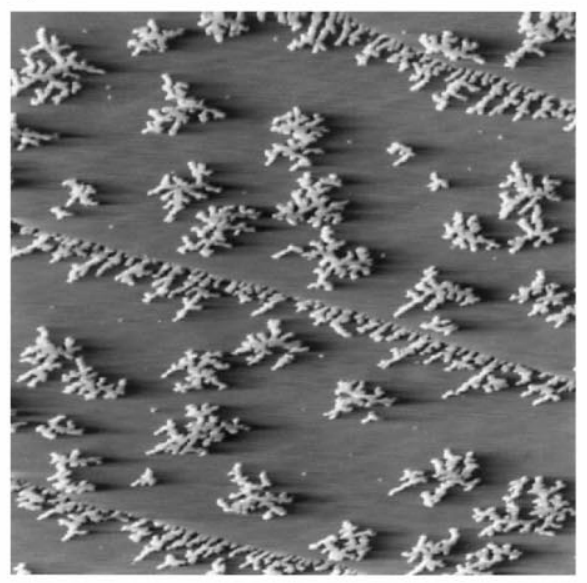

c)

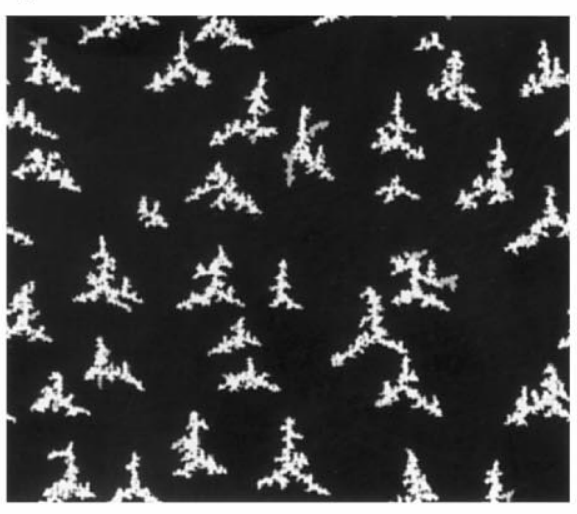

e)

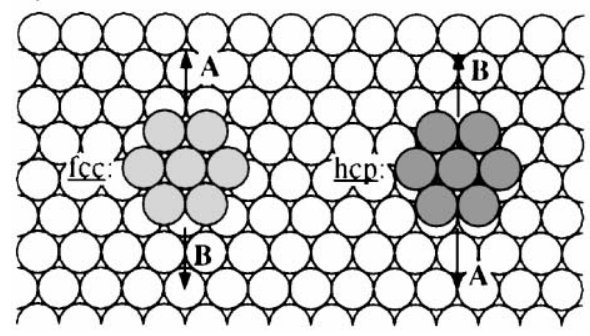

b)

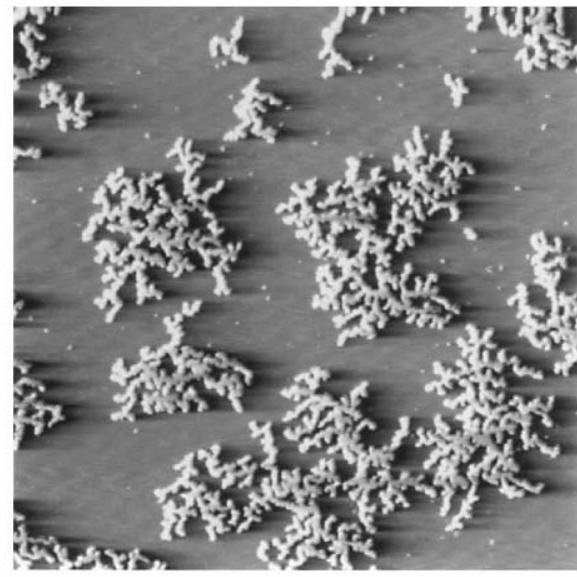

d)
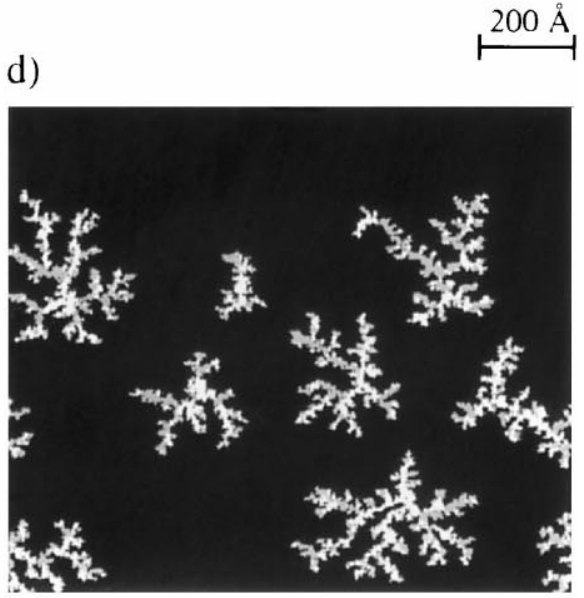

$200 \AA$

Fig. 37. Shape transition from dendritic to random fractal patterns driven by reduction of the growth speed. (a) and (b) STM images showing this transition for $\mathrm{Ag}$ deposition onto $\operatorname{Pt}(111)$ at $110 \mathrm{~K}\left(\Theta=0.12 \mathrm{ML}\right.$, (a) $F=1.1 \times 10^{-3} \mathrm{ML} / \mathrm{s}$, (b) $F=1.4 \times 10^{-5} \mathrm{ML} / \mathrm{s}$ ) (from Refs. [17,101]). (c) and (d) KMC-simulations performed with the experimental parameters of $\mathrm{Ag} / \mathrm{Pt}\left(\begin{array}{lll}1 & 1 & 1\end{array}\right)$ showing that dislocation formation and annihilation can reproduce the transition (hcp-areas gray, fcc-stacking areas white) (from Ref. [329]). (e) If a heptamer is situated at hcp-sites, the orientation of A- and B-steps turns by $60^{\circ}$ compared to fcc-site location. 
Physical evidence for creation of dislocations comes from the fact that $\mathrm{Ag}$ has a positive misfit of $m=4.33 \%$ on $\mathrm{Pt}$. Ag on $\mathrm{Pt}(111)$ is therefore under compressive strain which can favor dislocation formation, as experimentally observed for larger islands and submonolayer films [152,153]. The mechanism for generation of dislocation incorporated into the KMC simulations is closely related to a process associated with dendritic growth. We mentioned in connection with the growth scenario discussed in Fig. 35(a) that a dimer pointing away from an island can relax to an orientation associated with a higher coordination. This relexation involves a transition state where the atoms of the dimer are located on hcp-sites. From there the dimer can attach to the final fcc-sites with a small barrier. Due to compressive strain, however, the dimer atoms in the fcc-sites are slightly too close to the island and the dimer can detach again to the hcp-sites with a larger barrier, as suggested from EMT calculations. In the KMC simulations the detachment of a dimer from an island to the hcp-sites located next to it is allowed with a barrier of $0.33 \mathrm{eV}$ and relaxation back to fcc-sites with $0.20 \mathrm{eV}$. The first can lead to the creation of a partial dislocation if a third atom arrives before the dimer atoms relax back to fcc-sites. The other parameters were exactly those used to simulate dendritic growth for the same system (see Fig. 35). The process responsible for the creation of dislocations appears on the average once every $2 \mathrm{~min}$. This is the same amount of time needed to grow the clusters in Fig. 37(a). Therefore, only very few dislocations can form and remain stable under the typical flux, and dendritic growth appears. For the very low flux (Fig. 37(b)), the dimer can go to hcp-sites many times and almost half of the growth takes place on hcpsites. With each dislocation the preferred growth directions rotate by $60^{\circ}$ resulting in random growth.

In spite of the agreement between simulation and experiment furnished by this model, so far the formation of dislocations could not be proved experimentally. The detection of dislocations in branches

a)

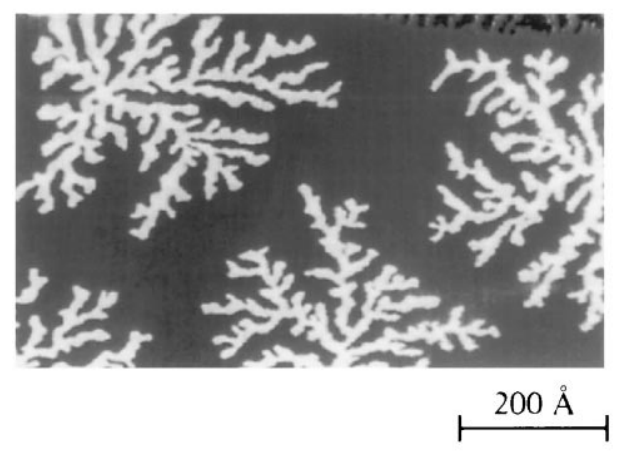

b)

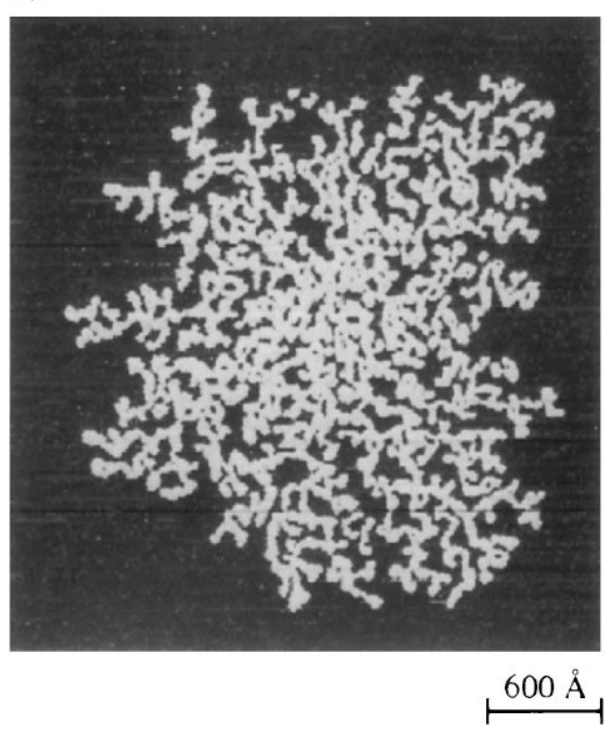

Fig. 38. Examples for random fractals following dendrites at elevated temperatures. (a) STM image of Au islands grown on $\mathrm{Ru}(0001)$ at $300 \mathrm{~K}\left(F=3.3 \times 10^{-3} \mathrm{ML} / \mathrm{s}, \Theta=0.30 \mathrm{ML}\right)$. The aggregates' branches spread out into random directions. Although they are significantly wider than those of the DLA clusters, the aggregate's fractal dimension is the DLA-value of 1.72 (from Ref. [263]). (b) STM image of a Ag fractal grown on $\operatorname{Pt}\left(\begin{array}{lll}1 & 1\end{array}\right)$ at $220 \mathrm{~K}$ similarly showing wide branches that frequently alter their growth direction $\left(F=1.1 \times 10^{-3} \mathrm{ML} / \mathrm{s}, \Theta=0.12 \mathrm{ML}\right)$ (from Ref. [330]). 
that are two atoms wide is a difficult experimental task. The dislocations detected in larger islands can be of thermodynamic origin as they are observed in compact islands after annealing or at room temperature growth. An experimental proof of kinetically generated transitions from fcc to hcp stacking is presently lacking. If there were such an activated generation of dislocations, it would become more prominent as the temperature is increased. This would also explain the random fractals generally formed at higher temperatures, two examples of which are shown in Fig. 38. The first example shows $\mathrm{Au}$ aggregates grown on $\mathrm{Ru}(0001)$ at room temperature. The second shows the formation of similar random fractals for $\mathrm{Ag} / \mathrm{Pt}\left(\begin{array}{lll}1 & 1 & 1\end{array}\right)$ at $220 \mathrm{~K}$. Both systems have considerable misfit supporting the idea of dislocations frequently altering the directions of preferred growth.

An alternative path towards an understanding of both the transition from dendritic towards random growth and of random growth at higher temperatures might lie in the transition to larger critical cluster sizes. For $\mathrm{Ag} / \mathrm{Pt}(111)$ a transition from $i=1$ to $i=2$ appears at $110 \mathrm{~K}$ for the typical deposition flux of $10^{-3} \mathrm{ML} / \mathrm{s}$ (see Fig. 9(a) in Section 3.3). Lowering the flux at $110 \mathrm{~K}$ drives the same transition. The dendrites in Fig. 37(a) are grown under conditions where dimers are stable, whereas during growth of the random fractals in Fig. 37(b) dimers were unstable. Dimer instability implies generally that all the one-fold coordinated atoms are unstable. As the mechanism for dendritic growth involves the relaxation of such one-fold atoms it may well be that their frequent detachment from islands perturbs dendritic growth. However, corner diffusion towards two-fold sites at steps should always be much faster than detachment of one-fold atoms, as the first was seen to be activated far before the latter. Therefore, we expect only a small amount of one-fold atoms to detach from islands before they can relax to stable sites via corner diffusion. However, the $\mathrm{Au} / \mathrm{Ru}\left(\begin{array}{llll}0 & 0 & 0 & 1\end{array}\right)$ clusters shown in Fig. 38(a) were also grown under $i>1$ (this can be concluded from total numbers of the island densities, assuming reasonable attempt frequencies). At present, the origin of random fractal patterns that often follow dendrite formation at increased temperatures or lower flux is not yet pinned down.

\subsubsection{Branch width and edge diffusion}

Both aggregates shown in Fig. 38 have an increased branch width with respect to the monoatomic branches of the DLA cluster and the di-atomic branches of dendrites. In order to point out this distinction they were labeled "fat" fractals [92]. The widening of branches was related to edge diffusion both by analytic models [88,318,319] and through KMC simulations [317]. The basic idea underlying these studies is that the lateral impingement rate competes with the rate for an adatom to scan the edge of a compact seed particle. The seed particle stays compact until the edges reach a critical width $(w)$ where both rates become comparable. At that point nucleation of protrusions at the edge can no longer be flattened out and the aggregate becomes unstable upon ramification through the MullinsSekerka instability (see Section 5.1). This instability argument defines the critical cluster size for ramification and equally the mean branch width taken on by the cluster after ramification. The models presented in the literature disagree on the exact dependence of $w$ on $D_{\mathrm{e}} / I$, however ( $I$ is the lateral impingement rate defined above, and $D_{\mathrm{e}}$ the $1 \mathrm{D}$ diffusion rate at the edge).

It was shown for $\mathrm{Ag} / \operatorname{Ag}\left(\begin{array}{lll}1 & 1 & 1\end{array}\right)$ and $\mathrm{Ag} / \mathrm{Pt}\left(\begin{array}{lll}1 & 1 & 1\end{array}\right)$ that the product of the branch width and $\sqrt{I}$ increases exponentially with deposition temperature within an extended temperature range [92]. This observation corroborates the model by Bartelt and Evans [317] predicting $w \approx\left(D_{\mathrm{e}} / I\right)^{1 / 2}$. From the quantitative analysis of the slopes for both systems, however, the barriers for edge diffusion come out smaller than those for terrace diffusion $\left(E_{\mathrm{e}}=125 \pm 10 \mathrm{meV}\right.$ for $\mathrm{Ag} / \mathrm{Pt}\left(\begin{array}{lll}1 & 1 & 1\end{array}\right)$ and $E_{\mathrm{e}}=65 \pm 10 \mathrm{meV}$ for $\mathrm{Ag} / \mathrm{Ag}\left(\begin{array}{lll}1 & 1 & 1\end{array}\right)$ ). Despite these small values the edge-diffusion rates were still realistic as extremely 


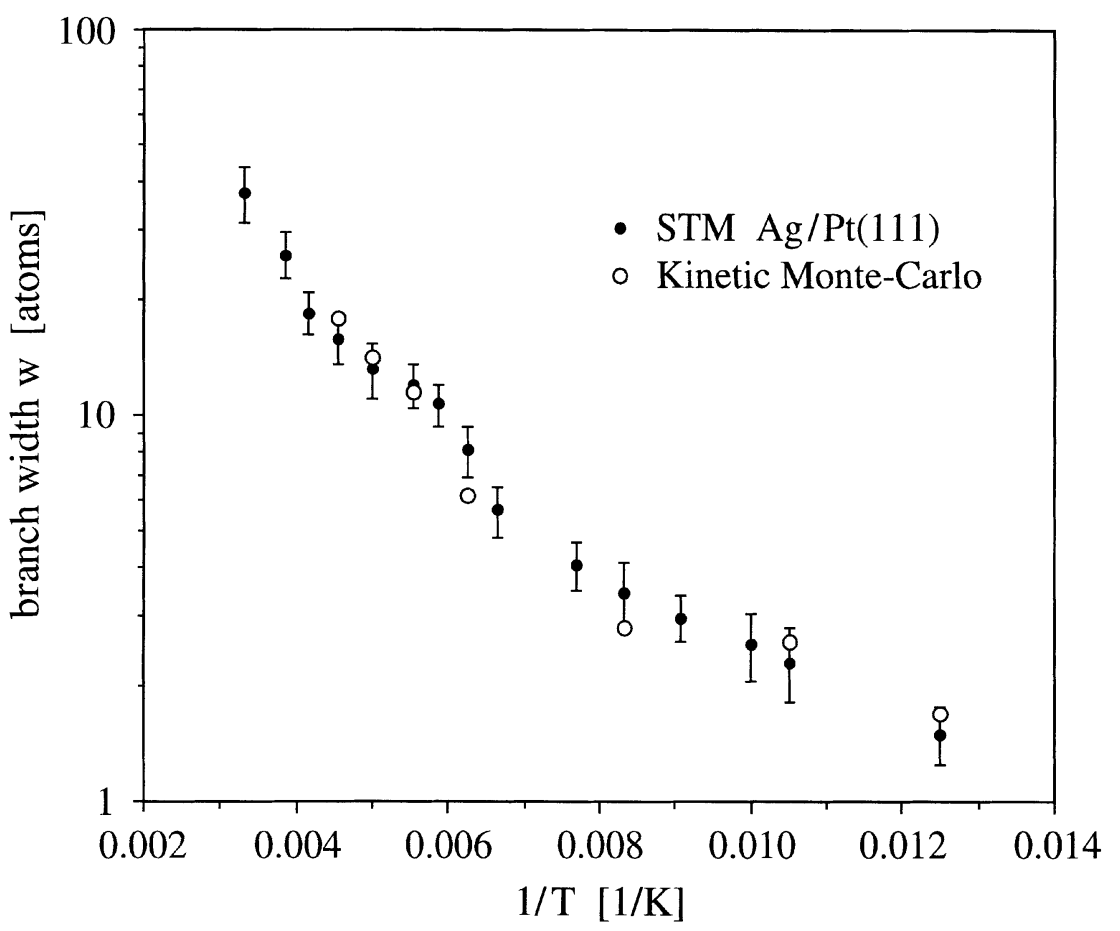

Fig. 39. Arrhenius plot of the branch width measured for $\mathrm{Ag} / \mathrm{Pt}\left(\begin{array}{ll}1 & 1\end{array}\right)$ in comparison with a KMC model incorporating essential microscopic details and thus yielding a reasonable value for the barrier for edge diffusion of $E_{\mathrm{e}}=370 \mathrm{meV}$ $\left(\nu_{0}=1 \times 10^{13} \mathrm{~Hz}\right)$ (from Ref. [329]).

small attempt frequencies compensated the small barriers. Although there is undoubtedly some truth in the instability criterion (it yields reasonable estimates for other systems [317,319]) the general difficulty with published models is that they neglect significant microscopic effects occurring at the island edge. For instance, the diffusion of an edge atom around a corner (corner crossing) involves the transition from two-fold to one-fold coordination. It therefore generally requires a higher activation energy than edge diffusion with the result that atoms can be trapped at their edges irrespective of the amount of edge diffusion. Since material transport away from a growing tip towards the cluster center involves corner crossing diffusion, these details are clearly important for a quantitative determination of edge-diffusion barriers.

Fig. 39 shows results from KMC simulations that take these important differences into account. The simulations incorporate edge diffusion (exclusively along A-steps as these are expected to have the smaller barrier for that system; the result, however, does not depend on this detail), diffusion from a two-fold edge site around a corner is not allowed, whereas diffusion from one-fold corner to edges has the typical small barriers (the barriers were the established parameters for $\operatorname{Ag} / \operatorname{Pt}\left(\begin{array}{lll}1 & 1 & 1\end{array}\right)$ ). It is evident that the branch width from the KMC-simulations fits the experimental values well. This agreement was obtained with an edge diffusion barrier of $E_{\mathrm{e}}=370 \mathrm{meV}$ and the same attempt frequency as the one for terrace diffusion of $\nu_{0}=1 \times 10^{13} \mathrm{~Hz}$. There is a clear discrepancy of this simulation with respect to the analysis with the theoretical models cited above. Bales and Chrzan [318] highlighted several weak points of the models presented in literature. In addition, differences between the models on how the 
transition from compact to ramified islands depends on the growth parameters were pointed out. The authors suggest a different dependence of the critical width for shape instability on the growth parameters which they verify with KMC simulations on a square lattice. The drawback of their approach is that comparison with experimental data is not straightforward and their model applies only to $i=1$. Continued effort will be needed to establish the link between edge diffusion and branch width in ramified island growth.

\subsubsection{Various polygonal island shapes}

Many epitaxial systems on hexagonal substrates reveal a direct transition from dendritic to compact islands upon increasing the deposition temperature. That way these systems bypass the "fat" branched random fractal stage. The compact islands are mostly triangles whose preferred orientation is set by the trigonal symmetry of the dendrites preceding at low temperatures. Examples for triangular islands with preferred orientation are $\mathrm{Co} / \mathrm{Ru}\left(0 \begin{array}{lll}0 & 0 & 1\end{array}\right)$ [263,331,332], $\mathrm{Co} / \mathrm{Pt}\left(\begin{array}{lll}1 & 1 & 1\end{array}\right)$ [333], and $\mathrm{Fe} / \mathrm{Au}\left(\begin{array}{lll}1 & 1 & 1\end{array}\right)$ where the triangles are less pronounced [245,246]. The most spectacular example is $\mathrm{Pt} / \mathrm{Pt}\left(\begin{array}{lll}1 & 1 & 1\end{array}\right)$ [279] which exhibits a transition from dendrites to a series of polygonal island shapes as displayed in Fig. 40. The dendrites formed at $200 \mathrm{~K}$ show preferred growth perpendicular to A-steps, with their triangular envelopes being formed by B-steps (Fig. 40(a)). Upon increasing the temperature triangles preferentially bound by A-steps are formed (Fig. 40(b)). These metamorphose via hexagons exhibiting both step orientations (Fig. 40(c)), into triangles that are bound by B-steps and hence oriented the other way around (Fig. 40(d)). Finally, the islands attain a quasi-hexagonal shape (Fig. 40(e)). This final island shape was demonstrated to be the thermodynamic equilibrium shape as it is independent of the cluster's history. It is obtained, either upon deposition at $710 \mathrm{~K}$, or by annealing to $710 \mathrm{~K}$ (see Figs. $40(\mathrm{e} 1)$ and (e2)). The different surface free energies of A- and B-steps express themselves in different lengths of these facets. ${ }^{53}$

Currently there are two contradictory interpretations for the transition between triangles of opposite orientation. We first focus on the original interpretation which suggests that the inversion of the edgediffusion rate with increasing temperature is responsible for the observed transition [279]. The rate of edge diffusion is reflected in the final island shape as those steps with fast edge diffusion lose their material to the other step orientation. Hence, the first propagate only slowly whereas the latter rapidly progress. As described above, it is the fundamental law of crystal growth that slowly growing facets prevail whereas the faster disappear. For $2 \mathrm{D}$ islands on hexagonal surfaces this implies that the step edges with the fast edge-diffusion characterize the final triangular islands. Accordingly, it was suggested for $\mathrm{Pt} / \mathrm{Pt}\left(\begin{array}{lll}1 & 1 & 1\end{array}\right)$ that diffusion along A-steps dominates at $400 \mathrm{~K}$ whereas at $600 \mathrm{~K}$ it is diffusion at B-steps which takes over.

Inversion of rates generally implies different barriers and different attempt frequencies. This leads to isokinetic temperatures which explain the intermediate hexagonal island shape in a straightforward way. At lower temperatures the barriers determine the rate, whereas the attempt frequencies gain importance at higher temperatures. In order to explain the results for $\mathrm{Pt} / \mathrm{Pt}\left(\begin{array}{lll}1 & 1 & 1\end{array}\right)$, diffusion along Bsteps was required to have a large barrier in order that it becomes slow at low temperature, and similarly B-step diffusion has to have a larger attempt frequency to overbalance A-step diffusion at high temperatures. The attempt frequencies and barriers for diffusion along the two facets can be inferred from a FIM study performed on $\operatorname{Pt}(311)$ and $\operatorname{Pt}(331)$ [334]. These values exactly match the

\footnotetext{
${ }^{53}$ Application of the Wulff theorem yields a step free energy ratio of $E_{\mathrm{B}} / E_{\mathrm{A}}=0.87 \pm 0.02$ for the close-packed $\{111\}$-facet vs. the open $\{100\}$ facet [321].
} 
a)

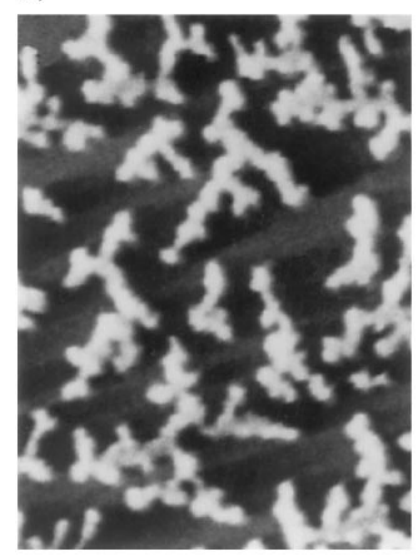

d)

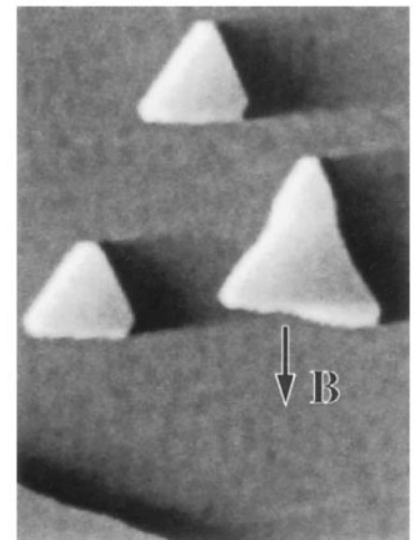

b)

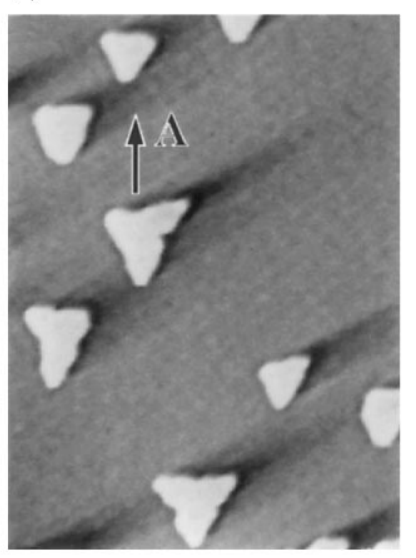

c)

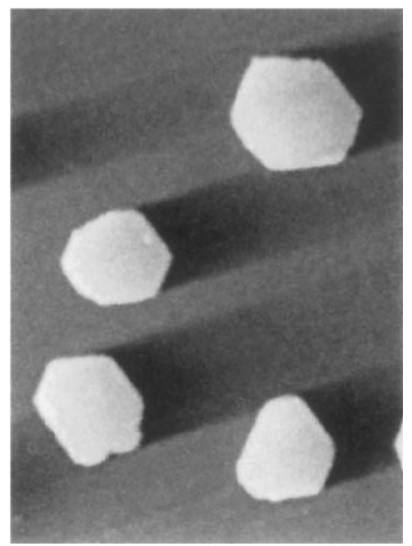

e)

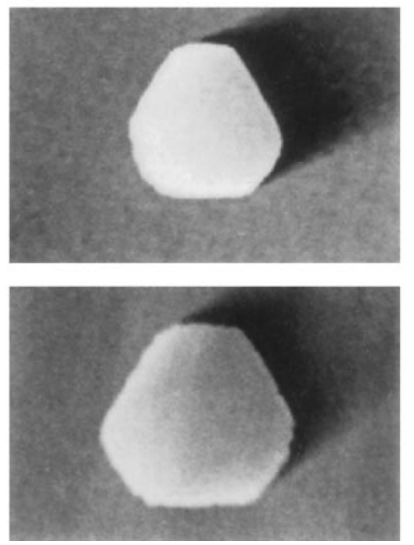

Fig. 40. The formation of dendritic and various polygonal islands during $\operatorname{Pt}\left(\begin{array}{lll}1 & 1 & 1\end{array}\right)$ homoepitaxy. The deposition temperatures $T$ are as follows: (a) $T=200 \mathrm{~K}\left(\Theta=0.2 \mathrm{ML}, 280 \times 400 \AA^{2}\right)$, (b) $T=400 \mathrm{~K}\left(\Theta=0.08 \mathrm{ML}, 1300 \times 1900 \AA^{2}\right),(\mathrm{c}) T=455 \mathrm{~K}$ $\left(\Theta=0.14 \mathrm{ML}, \quad 770 \times 1100 \AA^{2}\right), \quad(\mathrm{d}) \quad T=640 \mathrm{~K} \quad\left(\Theta=0.15 \mathrm{ML}, \quad 2300 \times 3300 \AA^{2}\right), \quad(\mathrm{e} 1) \quad T=710 \mathrm{~K} \quad(\Theta=0.08 \mathrm{ML}$, $1540 \times 1100 \AA^{2}$ ), and (e2) deposition at $T=455 \mathrm{~K}$ as in (c) and subsequent annealing to $710 \mathrm{~K}$ for $1 \mathrm{~min}(\Theta=0.08 \mathrm{ML}$, $\left.630 \times 900 \AA^{2}\right)\left(F=1 \times 10^{-2} \mathrm{ML} / \mathrm{s}\right)$ (from Ref. [279]).

requirements to explain the experimental observations. Diffusion along the channels on fcc(3 1 1) and fcc(3 31 ) surfaces is comparable to edge diffusion along A- and B-steps, respectively. The FIM studies performed on these surface orientations indicate as a general trend (for the elements investigated) that diffusion along B-steps has a larger barrier, but also a larger attempt frequency than diffusion along Asteps (for Rh see Ref. [335], for Ni [336], and for Ir [117]).

A physical basis for the difference in diffusion behavior along both step types comes from ab initio calculations performed for $\mathrm{Al}\left(\begin{array}{ll}1 & 1\end{array}\right)$ homoepitaxy $[132,337]$. Stumpf and Scheffler find that diffusion along the A-step occurs by normal hopping and has a small barrier. ${ }^{54}$ Diffusion along B-steps was found to proceed by an exchange and has a slightly larger barrier. Exchange involves a larger number of

\footnotetext{
${ }^{54}$ The geometric argument outlined above for corner diffusion similarly holds for edge diffusion. Therefore diffusion along Asteps is expected to have a smaller barrier due to the favorable location of hcp-hollows in front of these steps.
} 
a)

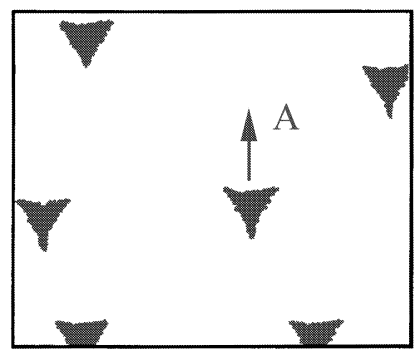

b)

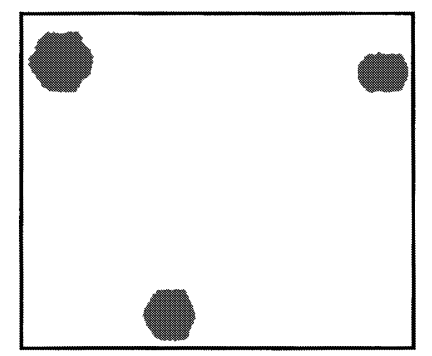

d)

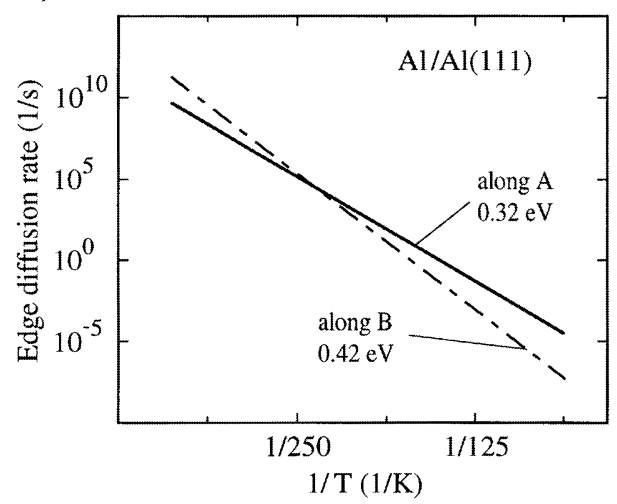

c)

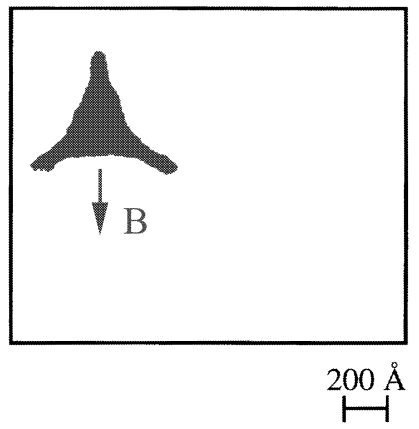

Fig. 41. KMC simulations performed with ab initio parameters of $\mathrm{Al} / \mathrm{Al}\left(\begin{array}{lll}1 & 1 & 1\end{array}\right)$ showing that a change in edge-diffusion rates for A- and B-steps yields a transition from triangles bound by A-steps via hexagons to triangles bounded by B-steps. Surface area in (a), (b) and (c) $1718 \times 1488 \AA^{2}(F=0.08 \mathrm{ML} / \mathrm{s}, \Theta=0.04 \mathrm{ML})$. (d) Arrhenius plot of the edge-diffusion rates along A- and B-steps $\left(E_{\mathrm{Ae}}=0.32 \mathrm{eV}, E_{\mathrm{Be}}=0.42 \mathrm{eV}\right)$ assuming $\nu_{0}, \mathrm{Ae}=2.5 \times 10^{12} \mathrm{~s}^{-1}$ and $\nu_{0, \mathrm{Be}}=2.5 \times 10^{14} \mathrm{~s}^{-1}$ (from Ref. [121]).

particles and different bonding configurations compared to hopping. This difference ${ }^{55}$ is thought to be the reason for the different attempt frequencies of both processes [121,323]. Ruggerone, Ratsch, and Scheffler recently showed that a difference in prefactor of two orders of magnitude can explain a transition from triangles via hexagons towards inverted triangles with a single parameter set (see Fig. 41). The concave sides of the island in Fig. 41(c) were also experimentally observed for both triangle orientations [338]. This shape results from a combination of the tip-effect and the increased island size. The adatom supply is largest at the triangle tips, when islands grow bigger this supply can no longer be equilibrated by edge diffusion and the edges grow faster close to the tip. Although the KMC simulations were performed with the ab initio activation energies of $\operatorname{Al}\left(\begin{array}{lll}1 & 1 & 1\end{array}\right)$ they generally suggest that inversion of edge-diffusion rates along the two types of steps can drive a similar shape transition as the one reported for $\mathrm{Pt} / \mathrm{Pt}\left(\begin{array}{lll}1 & 1 & 1\end{array}\right)$.

Despite the conclusive picture developed from the edge-diffusion argument and the tempting agreement with FIM measurements, Jacobsen et al. [328] recently suggested a quite different

\footnotetext{
${ }^{55}$ The authors pointed out that a compensation effect (see Section 3.6) would similarly increase the attempt frequency for the process with the larger barrier.
} 
a)

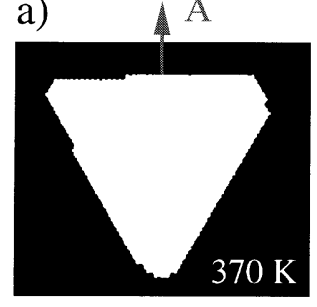

b)

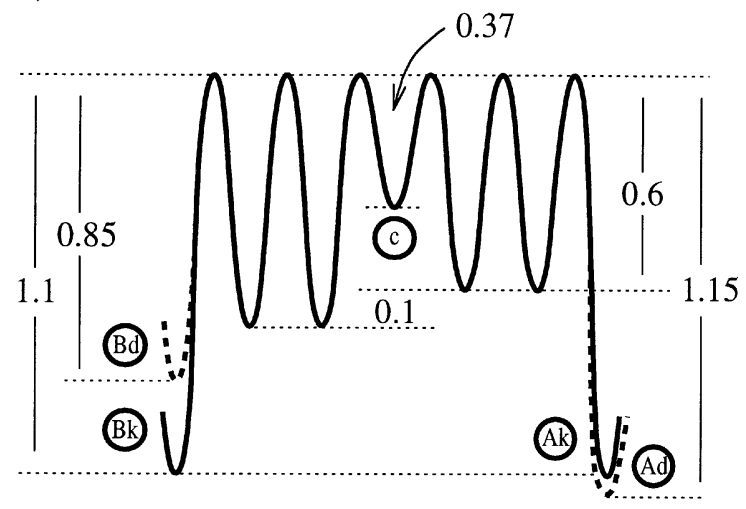

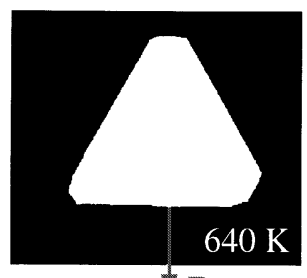

$1 \mathrm{~B}$
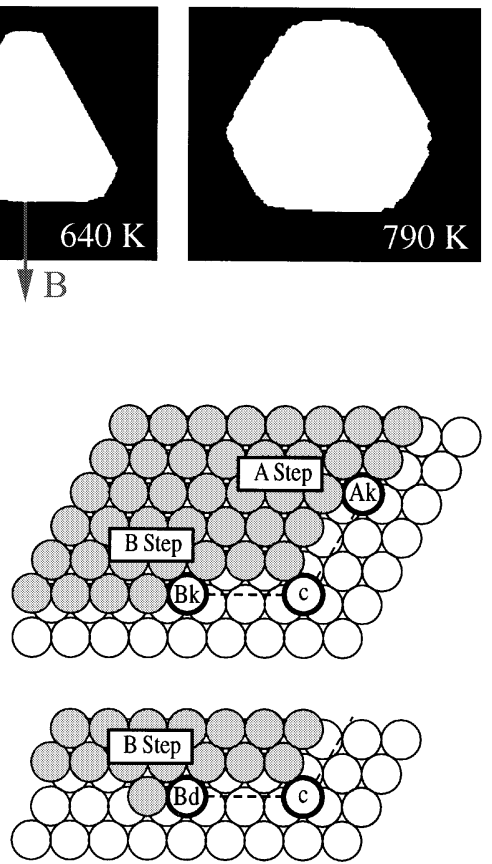

Fig. 42. KMC simulations explaining the shape transition for $\operatorname{Pt}\left(\begin{array}{lll}1 & 1 & 1\end{array}\right)$ homoepitaxy. (a) Island shapes obtained in the simulations as a function of temperature $\left(F=1 \times 10^{-3} \mathrm{ML} / \mathrm{s}, \Theta=0.05 \mathrm{ML}\right)$, island sizes from left to right: 1136,7000 , 18000 , and 11460 atoms, respectively. (b) Energy barriers (in eV) used in the KMC simulations. The solid curve shows the energy of an adatom along the indicated diffusion path whereas the dashed curves show how the kink dissociation barrier is changed if the atom is initially part of dimer (Ad and Bd on the A- and B-steps, respectively) (from Ref. [328]).

mechanism to drive the island shape transition for Pt(1 11 1) homoepitaxy. The model above does not account for an important microscopic detail, i.e. the diffusion around corners. As already pointed out this corner crossing presumably has a larger barrier than edge diffusion. More important, the direction of the net flux around the corner distinctively depends on the binding energy at both edges [328]. Jacobsen et al. note that the shape transition may not be achieved with the change in edge-diffusion rates alone.

The central point of their model is the binding energy of step atoms and of larger strings (including dimers) to the two step types. The energies can be inferred from Fig. 42(b). As required from energetic arguments dimers, or longer strings, are more stable at A-steps. In contrast to expectation, however, single atoms are assumed to be more strongly bound to B-steps. Edge diffusion has a larger barrier along B-steps than along A-steps, with the absolute values in agreement with FIM results. In contradistinction to FIM measurements, however, a single common attempt frequency is assumed for both (and for all other) processes.

This model is seen to reproduce the shape transition very convincingly (cf. Fig. 42(a)). At $400 \mathrm{~K}$ edge diffusion and corner crossing (having the same barrier in this study) are sufficiently fast that adatoms are more frequently at their preferred binding site. Whenever they encounter a second atom or attach to a kink they are immobilized as dissociation from kink sites is frozen at that temperature. This leads to a 
preferred population of B-steps which grow faster and hence disappear so that triangles with A-step edges prevail. At $600 \mathrm{~K}$ on the other hand, dissociation from kink sites is active and dimers and longer chains more likely form where they are more strongly bound, i.e. at A-steps. These steps therefore propagate faster and triangles with B-step edges remain.

The important parameter concerning shape transitions of compact islands is the net flux around steps. It is decisive in the growth speeds of the respective facets and determines which of them remains. The processes involved are corner crossing and dissociation from kink sites. In general, evaporation of step atoms towards the terrace plays a minor role as it has a larger barrier than any process at the edge.

The different diffusion processes at the island edge are all related in their specific way to the aggregate's shape. On hexagonal substrates, there are one-fold corner and two-fold step sites. Diffusion from the first to the latter is generally asymmetric for the two step types and leads, together with asymmetric attachment, to dendritic growth perpendicular to A-steps (the only exception to this rule presumably are systems with faster diffusion towards B-steps where random growth is expected). On square lattices, there are only one-fold edge sites. For both surface symmetries classical DLA aggregates are not expected as diffusion from one-fold sites has barriers similar to terrace diffusion. Therefore on square lattices islands are generally compact and on hexagonal lattices dendrites are observed down to lowest temperatures. On hexagonal lattices, a transition from dendrites to isotropic random patterns characterizes one class of systems. This transition is not yet fully understood and needs particular attention in future research. Edge diffusion at higher temperatures leads to coarsening of the branches. The aggregates stay ramified or irregular as long as corner crossing is frozen. Systems where this barrier is small evolve directly from dendritic to compact convex islands, whereas for systems with a large barrier for corner crossing aggregates might remain irregular up to very high temperatures. For systems with small barriers for corner crossing various compact island shapes can be realized as temperature increases. The understanding of these shapes and their transitions is still a challenge as the link between the pattern shape and microscopic details, such as binding energies or diffusion barriers at the steps, can be quite complex. Despite system specific complications several quite general microscopic processes and their influence on the pattern shape have become identified during recent years. This progress in 2D crystal growth might also be of use for 3D crystal growth and some ideas stemming from the surface studies reviewed in this section may possibly be transferred to more complicated systems.

\section{Summary and outlook}

Our understanding of the kinetics of nucleation and aggregation on metal surfaces considerably increased within recent years. From the experimental side a prominent contribution was made by variable temperature STM. Due to the combination of this technique with theoretical analysis, either in the framework of nucleation theory or kinetic Monte-Carlo simulations, we have new means to measure the activation energies for those atomic diffusion processes being most relevant to thin film and crystal growth. These observations supplement FIM measurements which so far were the most important and precise source of activation energies for diffusion of single particles. From the experimental material and the theory presented in this review we are presently approaching an understanding of epitaxial growth at the atomic level. This knowledge on the growth kinetics is essential for better growth control in MBE prepared samples. It can also be employed to tailor small structures with well-defined sizes and 
shapes through kinetically controlled growth. We gave several examples. Ostwald ripening can be employed to produce compact 2D islands of arbitrary size ranging from dimers to islands composed of 1000 atoms. Substrates with diffusion and sticking anisotropy can be used to grow 1D atomic chains with aspect ratios reaching 300. We are about to learn how the randomness inherent in deposition and diffusion can be reduced. The first examples discussed in Section 4.2 show remarkable size and distance uniformities lying beyond the best size distributions currently obtained for self-organized quantum dots grown by Stranski-Krastanov islanding. The approach of confined nucleation, in a dislocation network or moiré pattern, promises to be applicable to a wide range of heteroepitaxial systems. Diffusion mediated growth on isotropic substrates leads to a wealth of patterns that are now accessible with STM. For many patterns as well as shape transitions between them nature continues to keep most of its secrets. The open problems highlighted in Section 5 certainly deserve future attention.

The physical and chemical properties of low-dimensional structures are unique functions of their size and shape and differ largely from the behavior of bulk matter. Particularly fascinating phenomena occur if the nanostructures are subject to lateral boundary conditions on a length scale where quantum behavior prevails. Magnetic structures can be made out of non-magnetic elements [339]. The catalytic activity of small particles shows significant size effects [340,341]. The most striking example is gold. $\mathrm{Au}$ can become a good catalyst when present as small clusters [342] while as bulk matter it is the most inert material known to mankind [343]. Optical (see for instance plasmon excitations in small Ag particles [344,345]) and electronic properties (photoluminescence from quantum dots [346,347] and magnetism in metal clusters [348]) become widely adjustable through the particle size. Many of the physical and chemical properties can so far only be investigated with integrating techniques needing a considerable density of uniform particles. Since self-organized growth provides this density it can be a future way to examine and exploit new properties emerging from reduced particle dimensions.

\section{Acronyms}

$\begin{array}{ll}\text { CEM } & \text { corrected effective medium theory } \\ \text { DFT } & \text { density functional theory } \\ \text { DLA } & \text { diffusion limited aggregation } \\ \text { EAM } & \text { embedded atom method } \\ \text { EM } & \text { electron microscopy } \\ \text { EMT } & \text { effective medium theory } \\ \text { FIM } & \text { field ion microscopy } \\ \text { FP } & \text { first principles } \\ \text { GGA } & \text { generalized gradient correction } \\ \text { HAS } & \text { helium atom scattering } \\ \text { KMC } & \text { kinetic Monte-Carlo } \\ \text { LDA } & \text { local density approximation } \\ \text { LEED } & \text { low energy electron diffraction } \\ \text { LEIS } & \text { low energy ion scattering } \\ \text { LMTO } & \text { linear muffin tin orbital } \\ \text { MC } & \text { Monte-Carlo } \\ \text { MD } & \text { molecular dynamics }\end{array}$




$\begin{array}{ll}\text { RHEED } & \text { reflection high energy electron diffraction } \\ \text { SPA-LEED } & \text { spot profile analysis low energy electron diffraction } \\ \text { STM } & \text { scanning tunneling microscopy } \\ \text { TDS } & \text { thermal desorption spectroscopy } \\ \text { TEM } & \text { transmission electron microscopy } \\ \text { TST } & \text { transition state theory } \\ \text { VT-STM } & \text { variable-temperature scanning tunneling microscopy } \\ \text { XC } & \text { exchange correlation }\end{array}$

\section{Acknowledgements}

I am deeply indebted to my present and former collaborators within the Scanning Probe group in Lausanne, namely C. Boragno, K. Bromann, B. Fischer, A. Fricke, M. Giovannini, B. Müller, L. Nedelmann, H. Röder, and R. Schuster; their significant contribution to the experimental results presented in this review is gratefully acknowledged. I am indebted to J. Jacobsen, K.W. Jacobsen, P. Stoltze, and J. Nørskov for their hospitality during my stay in Denmark which launched a fruitful collaboration on the atomistic processes underlying pattern formation in metal epitaxy. The group of J. Nørskov also kindly provided their kinetic Monte-Carlo and effective medium theory computer codes. The first of which was adopted to particular experimental systems in Lausanne; a variety of results achieved with these calculations were presented in this review. I also gratefully acknowledge collaboration with G.S. Bales in analyzing our experimental results in terms of self-consistent meanfield nucleation theory. Illustrations to the present survey were kindly provided by C. Ratsch, P. Ruggerone, M. Scheffler, M. Schmidt, P. Varga, G.S. Bales, E. Lægsgaard, F. Besenbacher, T. Michely, A. Hitzke, and R.J. Behm. I am grateful to D. Purdie, F. Besenbacher, and J. Venables for carefully reading the manuscript. I am particularly indebted to $\mathrm{C}$-L. Bandelier who did an excellent job in preparing the figures of this review. Finally, my gratitude goes to Klaus Kern for his ongoing support of the work presented here, his stimulating discussions and keen suggestions.

\section{References}

[1] E. Bauer, Z. Krist. 110 (1958) 372.

[2] E. Bauer, J.H. v.d. Merwe, Phys. Rev. B 33 (1986) 3657.

[3] G. Binnig, H. Rohrer, C. Gerber, E. Weibel, Phys. Rev. Lett. 49 (1982) 57.

[4] G. Binnig, H. Rohrer, Helv. Phys. Acta 55 (1982) 726.

[5] G. Ehrlich, Appl. Phys. A 55 (1992) 403.

[6] G.L. Kellogg, Surf. Sci. Rep. 21 (1994) 1.

[7] T.T. Tsong, Atom-Probe Field Ion Microscopy, Cambridge University Press, Cambridge, 1990.

[8] G. Zinsmeister, Vacuum 16 (1966) 529.

[9] G. Zinsmeister, Thin Solid Films 7 (1971) 51.

[10] J.A. Venables, Phil. Mag. 17 (1973) 697.

[11] J.A. Venables, G.D.T. Spiller, M. Hanbücken, Rep. Prog. Phys. 47 (1984) 399.

[12] G.S. Bales, D.C. Chrzan, Phys. Rev. B 50 (1994) 6057.

[13] T. Vicsek, Fractal Growth Phenomena, World Scientific, Singapore, 1989.

[14] H. Takayasu, Fractals in the Physical Sciences, Manchester University Press, Manchester, NY, 1990. 
[15] T.A. Witten, L.M. Sander, Phys. Rev. Lett. 47 (1981) 1400.

[16] T.A. Witten, L.M. Sander, Phys. Rev. B 27 (1983) 5686.

[17] H. Brune, C. Romainczyk, H. Röder, K. Kern, Nature 369 (1994) 469.

[18] K. Bromann, C. Félix, H. Brune, W. Harbich, R. Monot, J. Buttet, K. Kern, Science 274 (1996) 956.

[19] D.M. Eigler, E.K. Schweizer, Nature 344 (1990) 524.

[20] M.F. Crommie, C.P. Lutz, D.M. Eigler, Science 262 (1993) 218.

[21] A.D. Kent, S. v. Molnar, S. Gider, D.D. Awschalom, J. Appl. Phys. 76 (1994) 6656.

[22] H. Röder, E. Hahn, H. Brune, J.P. Bucher, K. Kern, Nature 366 (1993) 141.

[23] D. Leonard, M. Krishnamurthy, C.M. Reaves, S.P. Denbaars, P.M. Petroff, Appl. Phys. Lett. 63 (1993) 3203.

[24] R. Nötzel, J. Tommyo, T. Tamamura, Nature 369 (1994) 131.

[25] F. Liu, M.G. Lagally, in: D.A. King, D.P. Woodruff (Eds.), Growth and Properties of Ultrathin Epitaxial Layers, vol. 8, Elsevier, Amsterdam, 1997, p. 149.

[26] M.G. Lagally, Phys. Today (November, 1993) 24.

[27] M.G. Lagally, Y.W. Mo, R. Kariotis, B.S. Swartzentruber, M.B. Webb, in: M.G. Lagally (Ed.), Kinetics of Ordering and Growth at Surfaces, Plenum Press, New York, 1990, p. 145.

[28] F. Besenbacher, L.P. Nielsen, P.T. Sprunger, in: D.A. King, D.P. Woodruff (Eds.), Growth and Properties of Ultrathin Epitaxial Layers, vol. 8, Elsevier, Amsterdam, 1997, p. 207.

[29] H. Brune, K. Kern, in: D.A. King, D.P. Woodruff (Eds.), Growth and Properties of Ultrathin Epitaxial Layers, vol. 8, Elsevier, Amsterdam, 1997, p. 149.

[30] R. Gomer, Rep. Prog. Phys. 53 (1990) 917.

[31] J. Ellis, J.P. Toennies, Phys. Rev. Lett. 70 (1993) 2118.

[32] G. Binnig, H. Fuchs, E. Stoll, Surf. Sci. 169 (1986) L295.

[33] M.L. Lozano, M.C. Tringides, Europhys. Lett. 30 (1995) 537.

[34] G. Ehrlich, Surf. Sci. 246 (1991) 1.

[35] B.S. Swartzentruber, Phys. Rev. Lett. 76 (1996) 459.

[36] T. Zambelli, J. Trost, J. Wintterlin, G. Ertl, Phys. Rev. Lett. 76 (1996) 795.

[37] M. Bott, M. Hohage, M. Morgenstern, T. Michely, G. Comsa, Phys. Rev. Lett. 76 (1996) 1304.

[38] J. Li, R. Berndt, W.D. Schneider, Phys. Rev. Lett. 76 (1996) 1888.

[39] M.R. Sørensen, K.W. Jacobsen, H. Jonsson, Phys. Rev. Lett. 77 (1996) 5076.

[40] Y.W. Mo, Phys. Rev. Lett. 71 (1993) 2923.

[41] T.R. Linderoth, S. Horch, E. Lægsgaard, I. Stensgaard, F. Besenbacher, Phys. Rev. Lett. 78 (1997) 4978.

[42] G. Ehrlich, F. Watanabe, Langmuir 7 (1991) 2555.

[43] F. Watanabe, G. Ehrlich, J. Chem. Phys. 96 (1992) 3191.

[44] S. Glasstone, K.J. Laidler, H. Eyring, The Theory of Rate Processes, McGraw-Hill, New York, 1941.

[45] D.E. Sanders, A.E. DePristo, Surf. Sci. 260 (1992) 116.

[46] G.A. Evangelakis, N.I. Papanicolaou, Surf. Sci. 347 (1996) 376.

[47] U. Kürpick, A. Kara, T.S. Rahman, Phys. Rev. Lett. 78 (1997) 1086.

[48] R. Gomer, in: V.T. Binh (Ed.), Surface Mobilities on Solid Materials, vol. 86, Plenum Press, New York, 1981 , p. 15.

[49] J. Frenkel, Z. Phys. 26 (1924) 117.

[50] M. Volmer, A. Weber, Z. Phys. Chem. 119 (1926) 277.

[51] W. Kossel, Nachr. Ges. Wiss. (Göttingen) Math.-Phys. K1. (1927) 135.

[52] I.N. Stranski, L. Krastanov, Sitzungsber. Akad. Wiss. Wien, Math.-naturwiss. Kl. IIb (1938) 797.

[53] B. Lewis, J.C. Anderson, Nucleation and Growth of Thin Films, Academic Press, New York, 1978.

[54] S. Stoyanov, D. Kashchiev, in: E. Kaldis (Ed.), Current Topics in Material Sciences, vol. 7, North-Holland, Amsterdam, 1981, p. 69.

[55] J.A. Venables, Phys. Rev. B 36 (1987) 4153.

[56] J. Villain, A. Pimpinelli, D.E. Wolf, Comments Condens. Matter Phys. 16 (1992) 1.

[57] J. Villain, A. Pimpinelli, L. Tang, D. Wolf, J. Phys. (Paris) I 2 (1992) 2107.

[58] J.A. Venables, Physica A 239 (1997) 35.

[59] H. Brune, H. Röder, C. Boragno, K. Kern, Phys. Rev. Lett. 73 (1994) 1955.

[60] M.J. Stowell, Phil. Mag. 26 (1972) 349.

[61] B. Lewis, Surf. Sci. 21 (1970) 289. 
[62] M.C. Bartelt, J.W. Evans, Phys. Rev. B 54 (1996) R17359.

[63] D.E. Wolf, in: M. Droz, K.J. McKane, J. Vannimenus, D.E. Wolf (Eds.), Scale Invariance, Interfaces, and NonEquilibrium Dynamics, Plenum Press, New York, 1994, p. 1.

[64] J. Jacobsen, PhD Thesis, Technical University of Denmark, Lyngby, 1996.

[65] A.F. Voter, Phys. Rev. B 34 (1986) 6819.

[66] J. Maddox, Nature 372 (1994) 403.

[67] M.C. Bartelt, J.W. Evans, Phys. Rev. B 46 (1992) 12675.

[68] M.C. Bartelt, J.W. Evans, Mater. Res. Soc. Symp. Proc. 312 (1993) 255.

[69] J.W. Evans, M.C. Bartelt, Surf. Sci. 284 (1993) L437.

[70] J.W. Evans, M.C. Bartelt, J. Vac. Sci. Technol. A 12 (1994) 1800.

[71] J.G. Amar, F. Family, P.M. Lam, Phys. Rev. B 50 (1994) 8781.

[72] C. Ratsch, A. Zangwill, P. Ŝmilauer, D.D. Vvedensky, Phys. Rev. Lett. 72 (1994) 3194.

[73] C. Ratsch, A. Zangwill, P. Ŝmilauer, Surf. Sci. 314 (1994) L937.

[74] L.H. Tang, J. Phys. (Paris) 13 (1993) 935.

[75] J.A. Stroscio, D.T. Pierce, Phys. Rev. B 49 (1994) 8522.

[76] Y.W. Mo, J. Kleiner, M.B. Webb, M.G. Lagally, Phys. Rev. Lett. 66 (1991) 1998.

[77] B. Müller, B. Fischer, L. Nedelmann, H. Brune, K. Kern, Phys. Rev. B 54 (1996) 17858.

[78] A. Zangwill, E. Kaxiras, Surf. Sci. 326 (1995) L483.

[79] D.D. Chambliss, K.E. Johnson, Phys. Rev. B 50 (1994) 5012.

[80] L. Bardotti, P. Jensen, A. Hoareau, M. Treilleux, B. Cabaud, Phys. Rev. Lett. 74 (1995) 4694.

[81] M.C. Bartelt, S. Günther, E. Kopatzki, R.J. Behm, J.W. Evans, Phys. Rev. B 53 (1966) 4099.

[82] L. Kuipers, R.E. Palmer, Phys. Rev. B 53 (1996) R7646.

[83] T.R. Linderoth, J.J. Mortensen, K.W. Jacobsen, E. Lægsgaard, I. Stensgaard, F. Besenbacher, Phys. Rev. Lett. 77 (1996) 87.

[84] S. Liu, L. Bönig, H. Metiu, Surf. Sci. 392 (1997) L56.

[85] H. Brune, S. Bales, C. Boragno, J. Jacobsen, K. Kern, Phys. Rev. B., to be published.

[86] J.A. Stroscio, D.T. Pierce, R.A. Dragoset, Phys. Rev. Lett. 70 (1993) 3615.

[87] C.M. Zhang, M.C. Bartelt, J.M. Wen, C.J. Jenks, J.W. Evans, P.A. Thiel, J. Cryst. Growth 174 (1997) 851.

[88] Z. Zhang, X. Chen, M.G. Lagally, Phys. Rev. Lett. 73 (1994) 1829.

[89] A. Brodde, G. Wilhelmi, D. Badt, H. Wengelnik, H. Neddermeyer, J. Vac. Sci. Technol. B 9 (1991) 920.

[90] B. Müller, L. Nedelmann, B. Fischer, H. Brune, J.V. Barth, K. Kern, Phys. Rev. Lett. 80, March 16 (1998).

[91] R.Q. Hwang, J. Schröder, C. Günther, R.J. Behm, Phys. Rev. Lett. 67 (1991) 3279.

[92] H. Röder, K. Bromann, H. Brune, K. Kern, Phys. Rev. Lett. 74 (1995) 3217.

[93] Q. Jiang, A. Chan, G.C. Wang, Phys. Rev. B 50 (1994) 11116.

[94] Q. Jiang, G.C. Wang, Surf. Sci. 324 (1995) 357.

[95] H. Dürr, J.F. Wendelken, J.K. Zuo, Surf. Sci. 328 (1995) L527.

[96] H.J. Ernst, F. Fabre, J. Lapujoulade, Phys. Rev. B 46 (1992) 1929.

[97] G. Ehrlich, J. Chem. Phys. 44 (1966) 1050.

[98] G. Ehrlich, F.G. Hudda, J. Chem. Phys. 44 (1966) 1039.

[99] K. Stolt, W.R. Graham, G. Ehrlich, J. Chem. Phys. 65 (1976) 3206.

[100] M. Bott, T. Michely, G. Comsa, Surf. Sci. 272 (1992) 161.

[101] H. Röder, H. Brune, J.P. Bucher, K. Kern, Surf. Sci. 298 (1993) 121.

[102] S. Günther, E. Kopatzki, M.C. Bartelt, J.W. Evans, R.J. Behm, Phys. Rev. Lett. 73 (1994) 553.

[103] J.G. Amar, F. Family, Phys. Rev. Lett. 74 (1995) 2066.

[104] M.C. Bartelt, L.S. Perkins, J.W. Evans, Surf. Sci. 344 (1995) L1193.

[105] W. Ostwald, Z. Phys. Chem. (Leipzig) 34 (1900) 495.

[106] C. Wagner, Z. Elektrochem. 65 (1961) 581.

[107] L.M. Lifshitz, V.V. Slyozov, J. Phys. Chem. Solids 19 (1961) 35.

[108] K. Morgenstern, G. Rosenfeld, G. Comsa, Phys. Rev. Lett. 76 (1996) 2113.

[109] W. Theis, N.C. Bartelt, R.M. Tromp, Phys. Rev. Lett. 75 (1995) 3328.

[110] S. Liu, L. Bönig, H. Metiu, Phys. Rev. B 52 (1995) 2907.

[111] G.L. Kellogg, A.F. Voter, Phys. Rev. Lett. 67 (1991) 622.

[112] G.L. Kellogg, Phys. Rev. Lett. 73 (1994) 1833. 
[113] K.W. Jacobsen, J.K. Nørskov, M.J. Puska, Phys. Rev. B 35 (1987) 7423.

[114] P. Stoltze, J. Phys.: Condens. Matter 6 (1994) 9495.

[115] S. Liu, Z. Zhang, J. Nørskov, H. Metiu, Surf. Sci. 321 (1994) 161.

[116] A. Bogicevic, private communication.

[117] S.C. Wang, G. Ehrlich, Surf. Sci. 239 (1990) 301.

[118] G.L. Kellogg, P. Feibelman, Phys. Rev. Lett. 64 (1990) 3143.

[119] C. Chen, T.T. Tsong, Phys. Rev. Lett. 64 (1990) 3147.

[120] P.J. Feibelman, Phys. Rev. Lett. 58 (1987) 2766.

[121] P. Ruggerone, C. Ratsch, M. Scheffler, in: D.A. King, D.P. Woodruff (Eds.), Growth and Properties of Ultrathin Epitaxial Layers, vol. 8, Elsevier, Amsterdam, 1997, p. 490.

[122] Z.P. Shi, Z. Zhang, A.K. Swan, J.F. Wendelken, Phys. Rev. Lett. 76 (1996) 4927.

[123] P. Jena, S.N. Khanna, B.K. Rao (Eds.), Physics and Chemistry of Finite Systems: From Clusters to Crystals, Kluwer, Dordrecht, 1992.

[124] B. Müller, L.P. Nedelmann, B. Fischer, H. Brune, J.V. Barth, K. Kern, D. Erdös, J. Wollschläger, Surf. Rev. Lett. 5 (1998), in press.

[125] L. Nedelmann, PhD Thesis, Ecole Polytechnique Fédérale, Lausanne, 1996.

[126] P.J. Feibelman, J.S. Nelson, G.L. Kellogg, Phys. Rev. B 49 (1994) 10548.

[127] S. Harris, P. Ŝmilauer, Phys. Rev. B 50 (1994) 7952.

[128] H. Brune, H. Röder, K. Bromann, K. Kern, Thin Solid Films 264 (1995) 230.

[129] S.C. Wang, G. Ehrlich, Phys. Rev. Lett. 70 (1993) 41.

[130] S.C. Wang, G. Ehrlich, Phys. Rev. Lett. 71 (1993) 4174.

[131] G. Vandoni, C. Félix, R. Monot, J. Buttet, W. Harbich, Surf. Sci. 320 (1994) L63.

[132] R. Stumpf, M. Scheffler, Phys. Rev. Lett. 72 (1994) 254.

[133] M. Villarba, H. Jónsson, Surf. Sci. 317 (1994) 15.

[134] J. Jacobsen, K.W. Jacobsen, P. Stoltze, J.K. Nørskov, Phys. Rev. Lett. 74 (1995) 2295.

[135] M.F. Sykes, M. Glen, J. Phys. A: Math. Gen. 9 (1976) 87.

[136] M.F. Sykes, D.S Gaunt, M. Glen, J. Phys. A: Math. Gen. 9 (1976) 97.

[137] G. Ehrlich, Brit. J. Appl. Phys. 15 (1964) 349.

[138] T. Gurney, F. Hutchinson, R.D. Young, J. Chem. Phys. 42 (1965) 3939.

[139] R.D. Young, D.S. Schubert, J. Chem. Phys. 42 (1965) 3943.

[140] W.F. Egelhoff, I. Jacob, Phys. Rev. Lett. 62 (1989) 921.

[141] J. Harris, B. Kasemo, Surf. Sci. 105 (1981) L281.

[142] H. Brune, J. Wintterlin, R.J. Behm, G. Ertl, Phys. Rev. Lett. 68 (1992) 624.

[143] J. Wintterlin, R. Schuster, G. Ertl, Phys. Rev. Lett. 77 (1996) 123.

[144] S.C. Wang, G. Ehrlich, J. Chem. Phys. 94 (1991) 4071.

[145] J.W. Evans, D.E. Sanders, P.A. Thiel, A.E. DePristo, Phys. Rev. B 41 (1990) 5410.

[146] S.V. Ghaisas, Surf. Sci. 223 (1989) 441.

[147] C. Roland, G.H. Gilmer, Phys. Rev. B 46 (1992) 13428.

[148] C. Ratsch, A. Zangwill, Appl. Phys. Lett. 63 (1993) 2348.

[149] H. Spjut, D.A. Faux, Surf. Sci. 306 (1994) 233.

[150] H. Brune, K. Bromann, H. Röder, K. Kern, J. Jacobsen, P. Stolze, K. Jacobsen, J. Nørskov, Phys. Rev. B 52 (1995) R14380.

[151] J.A. Meyer, P. Schmid, R.J. Behm, Phys. Rev. Lett. 74 (1995) 3864.

[152] K. Bromann, H. Brune, M. Giovannini, K. Kern, Surf. Sci. 388 (1997) L1107.

[153] S. Gestermann, M. Nohlen, M. Schmidt, K. Wandelt, Surf. Rev. Lett., in press.

[154] T. Härtel, U. Strüber, J. Küppers, Thin Solid Films 229 (1993) 163.

[155] H. Brune, H. Röder, C. Boragno, K. Kern, Phys. Rev. B 49 (1994) 2997.

[156] G. Rangelov, T. Fauster, U. Strüber, J. Küppers, Surf. Sci. 331-333 (1995) 948.

[157] H. Brune, K. Bromann, K. Kern, Mater. Res. Soc. Symp. Proc. 399 (1996) 213.

[158] C. Ratsch, A.P. Seitsonen, M. Scheffler, Phys. Rev. B 55 (1997) 6750.

[159] J.J. Mortensen, B. Hammer, O.H. Nielsen, K.W. Jacobsen, J.K. Nørskov, in: A. Okiji, H. Kasai, K. Makoshi (Eds.), Elementary Processes in Excitations and Reactions on Solid Surfaces, vol. 121, Springer, Berlin, 1996, p. 173. 
[160] M. Schroeder, D.E. Wolf, Surf. Sci. 375 (1997) 129.

[161] J. Tersoff, C. Teichert, M.G. Lagally, Phys. Rev. Lett. 76 (1996) 1675.

[162] C. Teichert, M.G. Lagally, L.J. Peticolas, J.C. Bean, J. Tersoff, Phys. Rev. B 53 (1996) 16334.

[163] K. Bromann, H. Brune, H. Röder, K. Kern, Phys. Rev. Lett. 75 (1995) 677.

[164] H. Röder, K, Bromann, H. Brune, K. Kern, Surf. Sci. 376 (1997) 13.

[165] J.K. Zuo, J.F. Wendelken, H. Dürr, C.L. Liu, Phys. Rev. Lett. 72 (1994) 3064.

[166] J.J.D. Miguel, A. Sánchez, E. Cebollada, J.M. Gallego, J. Ferrón, S. Ferrer, Surf. Sci. 189/190 (1987) 1062.

[167] M. Breeman, D.O. Boerma, Surf. Sci. 269/270 (1992) 224.

[168] L.S. Perkins, A.E. DePristo, Surf. Sci. 294 (1993) 67.

[169] J.W. Evans, M.C. Bartelt, in: M.C. Tringides (Ed.), Surface Diffusion: Atomistic and Collective Processes, NATO ASI, Plenum Press, New York, 1997, p. 197.

[170] M.H. Langelaar, D.O. Boerma, Surf. Sci. 352-354 (1996) 597.

[171] B. Müller, L. Nedelmann, B. Fischer, H. Brune, K. Kern, in: M. Tringides (Ed.), Surface Diffusion: Atomistic and Collective Processes, NATO ASI, Plenum Press, New York, 1997, p. 151.

[172] J. Wollschläger, D. Erdös, L. Nedelmann, B. Fischer, J.V. Barth, B. Müller, K. Kern, to be published.

[173] L. Olesen, E. Lægsgaard, I. Stensgaard, F. Besenbacher, J. Schiøtz, P. Stoltze, K.W. Jacobsen, J.K. Nørskov, Phys. Rev. Lett. 72 (1994) 2251.

[174] J. Jacobsen, L.P. Nielsen, F. Besenbacher, I. Stensgaard, E. Lægsgaard, T. Rasmussen, K.W. Jacobsen, J.K. Nørskov, Phys. Rev. Lett. 75 (1995) 489.

[175] J. Schiøtz, T. Rasmussen, K.W. Jacobsen, O.H. Nielsen, Phil. Mag. Lett. 74 (1996) 339.

[176] L. Hansen, P. Stoltze, K.W. Jacobsen, J.K. Nørskov, Phys. Rev. B 44 (1991) 6523.

[177] C.L. Liu, J.M. Cohen, J.B. Adams, A.F. Voter, Surf. Sci. 253 (1991) 334.

[178] C.L. Liu, Surf. Sci. 316 (1994) 294.

[179] G. Boisvert, L.J. Lewis, M.J. Puska, R.M. Nieminen, Phys. Rev. B 52 (1995) 9078.

[180] G. Boisvert, L.J. Lewis, Phys. Rev. B 54 (1996) 2880.

[181] L.S. Perkins, A.E. DePristo, Surf. Sci. 319 (1994) 225.

[182] P.J. Feibelman, Surf. Sci. 313 (1994) L801.

[183] G.W. Jones, J.M. Marcano, J.K. Nørskov, J.A. Venables, Phys. Rev. Lett. 65 (1990) 3317.

[184] G. Rosenfeld, N.N. Lipkin, W. Wulfhekel, J. Kliewer, K. Morgenstern, B. Poelsema, G. Comsa, Appl. Phys. A 61 (1995) 455.

[185] E.Z. Luo, J. Wollschläger, F. Wegner, M. Henzler, Appl. Phys. A 60 (1995) 19.

[186] K. Kyono, A. Gölzhäuser, G. Ehrlich, Surf. Sci. 397 (1998) 191.

[187] T. Michely, private communication.

[188] W. Wulfhekel, N.N. Lipkin, J. Kliewer, G. Rosenfeld, L.C. Jorritsma, B. Poelsema, G. Comsa, Surf. Sci. 348 (1996) 227.

[189] H. Brune, K. Bromann, K. Kern, in: M.C. Tringides (Ed.), Surface Diffusion: Atomistic and Collective Processes, NATO ASI, Plenum Press, New York, 1997, p. 135.

[190] B. Fischer, H. Brune, A. Fricke, J.V. Barth, K. Kern, to be published.

[191] B. Fischer, J. Weckesser, J. Barth, H. Brune, K. Kern, to be published.

[192] W. Meyer, H. Neldel, Z. Tech. Phys. 12 (1937) 588.

[193] G. Boisvert, L.J. Lewis, A. Yelon, Phys. Rev. Lett. 75 (1995) 469.

[194] J.W. Niemantsverdriet, K. Wandelt, J. Vac. Sci. Technol. A 6 (1988) 757.

[195] A.M. de Jong, J.W. Niemantsverdriet, Surf. Sci. 233 (1990) 355.

[196] D.P. Almond, A.R. West, Solid State Ionics 23 (1987) 27.

[197] A.F. Voter, J.D. Doll, J. Chem. Phys. 80 (1984) 5832.

[198] D.C. Senft, G. Ehrlich, Phys. Rev. Lett. 74 (1995) 294.

[199] D.E. Sanders, A.E. DePristo, Surf. Sci. 264 (1992) L169.

[200] F. Watanabe, G. Ehrlich, J. Chem. Phys. 95 (1991) 6075.

[201] S. Liu, L. Bönig, J. Detch, H. Metiu, Phys. Rev. Lett. 74 (1995) 4495.

[202] Y.W. Mo, B.S. Swartzentruber, B. Kariotis, M.B. Webb, M.G. Lagally, Phys. Rev. Lett. 63 (1989) 2393.

[203] Y.W. Mo, J. Kleiner, M.B. Webb, M.G. Lagally, Surf. Sci. 268 (1992) 275.

[204] C. Pearson, M. Krueger, E. Ganz, Phys. Rev. Lett. 76 (1996) 2306.

[205] H. Metiu, Y.T. Lu, Z. Zhang, Science 255 (1992) 1088. 
[206] J.P. Bucher, E. Hahn, P. Fernandez, C. Massobrio, K. Kern, Europhys. Lett. 27 (1994) 473.

[207] M.C. Bartelt, J.W. Evans, Europhys. Lett. 21 (1993) 99.

[208] J.D. Wrigley, G. Ehrlich, Phys. Rev. Lett. 44 (1980) 661.

[209] G.L. Kellogg, Phys. Rev. Lett. 67 (1991) 216.

[210] C. Chen, T.T. Tsong, Phys. Rev. Lett. 66 (1991) 1610.

[211] T.T. Tsong, C. Chen, Phys. Rev. B 43 (1991) 2007.

[212] C. Chen, T.T. Tsong, Phys. Rev. B 46 (1992) 7803.

[213] L.P. Nielsen, F. Besenbacher, E. Lægsgaard, I. Stensgaard, C. Engdahl, P. Stoltze, K.W. Jacobsen, J.K. Nørskov, Phys. Rev. Lett. 71 (1993) 754.

[214] M.B. Hugenschmidt, A. Hitzke, R.J. Behm, Phys. Rev. Lett. 76 (1996) 2535.

[215] S. Rousset, S. Chiang, D.E. Fowler, D.D. Chambliss, Phys. Rev. Lett. 69 (1992) 3200.

[216] Y. Li, M.C. Bartelt, J.W. Evans, N. Waelchli, E. Kampshoff, K. Kern and A.E. DePristo, Phys. Rev. B 56 (1997) 12539.

[217] N. Waelchli, E. Kampshoff, K. Kern, to be published.

[218] N. Waelchli, PhD Thesis, Ecole Polytechnique Fédérale, Lausanne, 1997.

[219] M.A. Van Hove, R.J. Koestner, P.C. Stair, J.P. Bibérain, L.L. Kesmodel, I. Bartos, G.A. Somorjai, Surf. Sci. 103 (1981) 189.

[220] J.F. Wendelken, D.M. Zehner, Surf. Sci. 71 (1978) 178.

[221] H. Melle, E. Menzel, Z. Naturforsch. 33a (1978) 282.

[222] K.H. Rieder, T. Engel, R.H. Swendsen, M. Manninen, Surf. Sci. 127 (1983) 223.

[223] G.K. Binnig, H. Rohrer, C. Gerber, E. Stoll, Surf. Sci. 144 (1984) 321.

[224] K. Yamazaki, K. Takayanagi, Y. Tanishiro, K. Yagi, Surf. Sci. 199 (1988) 595.

[225] S.G.J. Mochrie, D.M. Zehner, B.M. Ocko, D. Gibbs, Phys. Rev. Lett. 64 (1990) 2925.

[226] D. Gibbs, B.M. Ocko, D.M. Zehner, S.G.J. Mochrie, Phys. Rev. B 42 (1990) 7330.

[227] B.M. Ocko, D. Gibbs, K.G. Huang, D.M. Zehner, S.G.J. Mochrie, Phys. Rev. B 44 (1991) 6429.

[228] D.L. Albernathy, S.G.J. Mochrie, D.M. Zehner, G. Grübel, D. Gibbs, Phys. Rev. B 45 (1992) 9272.

[229] J.V. Barth, PhD Thesis, Freie Universität, Berlin, 1992.

[230] A. Pimpinelli, J. Villain, D.E. Wolf, Phys. Rev. Lett. 69 (1992) 985.

[231] Y.W. Mo, J. Kleiner, M.B. Webb, M.G. Lagally, Phys. Rev. Lett. 69 (1992) 986.

[232] T. Michely, M. Hohage, S. Esch, G. Comsa, Surf. Sci. 349 (1996) L89.

[233] M. Bott, M. Hohage, T. Michely, G. Comsa, Phys. Rev. Lett. 70 (1993) 1489.

[234] R. Kunkel, B. Poelsema, L.K. Verheij, G. Comsa, Phys. Rev. Lett. 65 (1990) 733.

[235] H. Brune, M. Giovannini, K. Bromann, K. Kern, Nature, in press.

[236] J. Perdereau, J.P. Bibérian, G.E. Rhead, J. Phys. F 4 (1974) 798.

[237] Y. Tanishiro, H. Kanamori, K. Takayanagi, K. Yagi, G. Honjo, Surf. Sci. 111 (1981) 395.

[238] J.V. Barth, H. Brune, G. Ertl, R.J. Behm, Phys. Rev. B 42 (1990) 9307.

[239] U. Harten, A.M. Lahee, J.P. Toennies, C. Wöll, Phys. Rev. Lett. 54 (1985) 2619.

[240] S. Narasimhan, D. Vanderbilt, Phys. Rev. Lett. 69 (1992) 1564.

[241] D.D. Chambliss, R.J. Wilson, S. Chiang, Phys. Rev. Lett. 66 (1991) 1721.

[242] D.D. Chambliss, R.J. Wilson, S. Chiang, J. Vac. Sci. Technol. B 9 (1993) 933.

[243] D.D. Chambliss, K.E. Johnson, R.J. Wilson, S. Chiang, J. Magn. Magn. Mater. 121 (1993) 1.

[244] J.A. Meyer, J.D. Baikie, E. Kopatzki, R.J. Behm, Surf. Sci. 365 (1996) L647.

[245] B. Voigtländer, G. Meyer, N.M. Amer, Surf. Sci. 255 (1991) L529.

[246] J.A. Stroscio, D.T. Pierce, R.A. Dragoset, P.N. First, J. Vac. Sci. Technol. A 10 (1992) 1981.

[247] B. Voigtländer, G. Meyer, N.M. Amer, Phys. Rev. B 44 (1991) 10354.

[248] E.I. Altman, R.J. Colton, Surf. Sci. 304 (1994) L400.

[249] M.M. Dovek, C.A. Lang, J. Nogami, C.F. Quate, Phys. Rev. B 40 (1989) 11973.

[250] D.D. Chambliss, R.J. Wilson, J. Vac. Sci. Technol. B 9 (1991) 928.

[251] M. Ruff, M. Hugenschmidt, R.J. Behm, to be published.

[252] C.A. Lang, M.M. Dovek, J. Nogami, C.F. Quate, Surf. Sci. 224 (1989) 2947.

[253] B. Fischer, J.V. Barth, A. Fricke, L. Nedelmann, K. Kern, Surf. Sci. 389 (1997) 366.

[254] M.A. Krzyzowski, PhD Thesis, Universität Bonn, 1995.

[255] J. Jacobsen, K.W. Jacobsen, P. Stoltze, Surf. Sci. 317 (1994) 8. 
[256] G.O. Pötschke, R.J. Behm, Phys. Rev. B 44 (1991) 1442.

[257] C. Günther, J. Vrijmoeth, R.Q. Hwang, R.J. Behm, Phys. Rev. Lett. 74 (1995) 754.

[258] P.A. Mulheran, J.A. Blackman, Phil. Mag. Lett. 72 (1995) 55.

[259] P.A. Mulheran, J.A. Blackman, Phys. Rev. B 53 (1996) 10261.

[260] S. Fafard, R. Leon, D. Leonard, J.L. Merz, P.M. Petroff, Phys. Rev. B 52 (1995) 5752.

[261] K.R. Heim, S.T. Coyle, G.G. Hembree, J.A. Venables, M.R. Scheinfein, J. Appl. Phys. 80 (1996) 1161.

[262] J. Barth, R.J. Behm, G. Ertl, Surf. Sci. 341 (1995) 62.

[263] C. Günther, S. Günther, E. Kopatzki, R.Q. Hwang, J. Schröder, J. Vrijmoeth, R.J. Behm, Ber. Bunsenges. Phys. Chem. 97 (1993) 522.

[264] M. Böhringer, P. Molinas-Mata, E. Artacho, J. Zegenhagen, Phys. Rev. B 51 (1995) 9965.

[265] E. Artacho, P. Moliǹas-Mata, M. Böhringer, J. Zegenhagen, G.E. Franklin, J.R. Patel, Phys. Rev. B 51 (1995) 9952.

[266] M. Horn-von-Hoegen, A.A. Falou, H. Pietsch, B.H. Müller, M. Henzler, Surf. Sci. 298 (1993) 29.

[267] T.A. Land, T. Michely, R.J. Behm, J.C. Hemminger, G. Comsa, Surf. Sci. 264 (1992) 261.

[268] T. Wiederholt, H. Brune, J. Wintterlin, R.J. Behm, G. Ertl, Surf. Sci. 324 (1994) 91.

[269] M. Böhringer, Q. Jiang, R. Berndt, W.D. Schneider, J. Zegenhagen, Surf. Sci. 367 (1996) 245.

[270] L.P. Nielsen, E. Lægsgaard, I. Stensgaard, F. Besenbacher, to be published.

[271] L. Vegard, Z. Phys. 5 (1921) 17.

[272] C. Nagl, M. Pinczolits, M. Schmid, P. Varga, I.K. Robinson, Phys. Rev. B 52 (1995) 16796.

[273] K.S. Liang, K.L. D’Amico, C.H. Lee, E.Y. Sheu, Phys. Rev. Lett. 65 (1990) 3025.

[274] C.H. Lee, E.Y. Sheu, K.S. Liang, K.L. D’Amico, W.N. Unertl, Appl. Phys. A 51 (1990) 191.

[275] C. Nagl, M. Schmid, P. Varga, Surf. Sci. 369 (1996) 159.

[276] C. Mottet, G. Tréglia, B. Legrand, Phys. Rev. B 46 (1992) 16018.

[277] S.M. Foiles, Surf. Sci. 292 (1993) 5.

[278] R.Q. Hwang, J.C. Hamilton, J.L. Stevens, S.M. Foiles, Phys. Rev. Lett. 75 (1995) 4242.

[279] T. Michely, M. Hohage, M. Bott, G. Comsa, Phys. Rev. Lett. 70 (1993) 3943.

[280] H. Brune, K. Bromann, J. Jacobsen, K. Jacobsen, P. Stoltze, J. Nørskov, K. Kern, Surf. Sci. 349 (1996) L115.

[281] H. Brune, K. Bromann, K. Kern, J. Jacobsen, K. Jacobsen, P. Stoltze, J. Nørskov, Mater. Res. Soc. Symp. Proc. 407 (1996) 379.

[282] M. Hohage, M. Bott, M. Morgenstern, Z. Zhang, T. Michely, G. Comsa, Phys. Rev. Lett. 76 (1996) 2366.

[283] Y. Furukawa, Chem. unserer Zeit 2 (1997) 58.

[284] J. Kepler, De Nive Sexangula, Frankfurt, 1611.

[285] R. Descartes, Dicours de la Méthode. Pour bien conduire, sa raison, et chercher la vérité dans les sciences. Plus la Dioptrique, les Météores et la Géométrie. Qui sont les essais de cette Méthode, Jan Maire, Leyden, 1637.

[286] F.C. Frank, J. Glaci. 13 (1974) 535.

[287] W.A. Bentley, W.J. Humphreys, Snow Crystals, McGraw-Hill, New York, 1931.

[288] U. Nakaya, Snow Crystals, Natural and Artificial, Harvard University Press, Cambridge, 1954.

[289] J.S. Langer, Rev. Mod. Phys. 52 (1980) 1.

[290] P. Davies, The New Physics, Cambridge University Press, Cambridge, 1989.

[291] B.B. Mandelbrot, Les Objets Fractals: Forme, Hasard et Dimension, Flamerion, Paris, 1975.

[292] P. Meakin, in: C. Domb, J.L. Lebowitz (Eds.), Phase Transitions and Critical Phenomena, vol. 12, Academic Press, New York, 1988, p. 335.

[293] E. Ben-Jacob, O. Schochet, A. Tenebaum, I. Cohen, A. Czirok, T. Vicsek, Nature 368 (1994) 46.

[294] Niemeyer, Phys. Rev. Lett. 52 (1984) 1033.

[295] B.B. Mandelbrot, The Fractal Geometry of Nature, Freeman, New York, 1982.

[296] G. Dietler, Y.-C. Zhang, Physica A 191 (1992) 213.

[297] H.E. Stanley, N. Ostrowsky (Eds.), On Growth and Form, Fractal and Non-Fractal Patterns in Physics, NATO ASI Ser. Nijhoff, Dordrecht, 1986.

[298] J.C. Russ, Fractal Surfaces, Plenum Press, New York, 1994.

[299] A.L. Barabási, H.E. Stanley, Fractal Concepts in Surface Growth, Cambridge University Press, New York, 1995.

[300] Y. Sawada, A. Dougherty, J.P. Gollub, Phys. Rev. Lett. 56 (1986) 1260.

[301] D. Grier, E. Ben-Jacob, R. Clarke, L.M. Sander, Phys. Rev. Lett. 56 (1986) 1264.

[302] H.S.S. Hele-Shaw, Nature 58 (1898) 34. 
[303] H. Brune, H. Röder, C. Romainczyk, C. Boragno, K. Kern, Appl. Phys. A 60 (1995) 167.

[304] J. Nittmann, Nature 314 (1985) 141.

[305] E. Ben-Jacob, R. Godbey, N.D. Goldenfeld, J. Koplik, H. Levine, T. Mueller, L.M. Sander, Phys. Rev. Lett. 55 (1985) 1315.

[306] V. Horváth, T. Vicsek, J. Kertész, Phys. Rev. A 35 (1987) 2353.

[307] A. Buka, J. Kertész, T. Vicsek, Nature 323 (1986) 424.

[308] J. Dalton, A New System of Chemical Philosophy, Manchester, 1808.

[309] J. Nittmann, H.E. Stanley, Nature 321 (1986) 663.

[310] J. Nittmann, H.E. Stanley, J. Phys. A: Math. Gen. 20 (1997) L981.

[311] P. Meakin, Phys. Rev. A 27 (1983) 1495.

[312] J.P. Eckmann, P. Meakin, I. Procaccia, R. Zeitak, Phys. Rev. Lett. 65 (1990) 52.

[313] J. Kertész, T. Vicsek, J. Phys. A: Math. Gen. 19 (1986) L257.

[314] P. Meakin, Phys. Rev. A 36 (1987) 332.

[315] J.P. Eckmann, P. Meakin, I. Procaccia, R. Zeitak, Phys. Rev. A 39 (1989) 3185.

[316] B. Gleich, R.J. Behm, to be published.

[317] M.C. Bartelt, J.W. Evans, Surf. Sci. 314 (1994) L829.

[318] G.S. Bales, D.C. Chrzan, Phys. Rev. Lett. 74 (1995) 4879.

[319] A. Pimpinelli, J. Villain, D.E. Wolf, J. Phys. (Paris) 3 (1993) 447.

[320] S.C. Wang, G. Ehrlich, Phys. Rev. Lett. 67 (1991) 2509.

[321] T. Michely, G. Comsa, Surf. Sci. 256 (1991) 217.

[322] W.W. Mullins, R.F. Sekerka, J. Appl. Phys. A 34 (1963) 323.

[323] C. Ratsch, P. Ruggerone, M. Scheffler, in: M.C. Tringides (Ed.), Surface Diffusion: Atomistic and Collective Processes, NATO ASI, Plenum Press, New York, 1997, p. 83.

[324] E. Kopatzki, S. Günther, W. Nichtl-Pecher, R.J. Behm, Surf. Sci. 284 (1993) 154.

[325] E. Hahn, E. Kampshoff, N. Wälchli, K. Kern, Phys. Rev. Lett. 74 (1995) 1803.

[326] J.M. Wen, J.W. Evans, M.C. Bartelt, J.W. Burnett, P.A. Thiel, Phys. Rev. Lett. 76 (1996) 652.

[327] S. Liu, Z. Zhang, G. Comsa, H. Metiu, Phys. Rev. Lett. 71 (1993) 2967.

[328] J. Jacobsen, K.W. Jacobsen, J.K. Nørskov, Surf. Sci. 359 (1996) 37.

[329] H. Brune, J. Jacobsen, K. Kern, to be published.

[330] K. Bromann, H. Brune, K. Kern, unpublished results, 1996.

[331] R.Q. Hwang, C. Günther, J. Schröder, S. Günther, E. Kopatzki, R.J. Behm, J. Vac. Sci. Technol. A 10 (1992) 1970.

[332] J. Vrijmoeth, C. Günther, J. Schröder, R.Q. Hwang, R.J. Behm, in: R.F.C. Farrow (Ed.), Magnetism and Structure in Systems of Reduced Dimension, Plenum Press, New York, 1993, p. 55.

[333] C. Boragno, H. Röder, H. Brune, K. Kern, to be published.

[334] D.W. Bassett, P.R. Webber, Surf. Sci. 70 (1978) 520.

[335] G. Ayrault, G. Ehrlich, J. Chem. Phys. 60 (1974) 281.

[336] R.T. Tung, W.R. Graham, Surf. Sci. 97 (1980) 73.

[337] R. Stumpf, M. Scheffler, Phys. Rev. B 53 (1996) 4958.

[338] T. Michely, M. Hohage, G. Comsa, in: M.C. Tringides (Ed.), Surface Diffusion: Atomistic and Collective Processes, NATO ASI, Plenum Press, New York, 1997, p. 125.

[339] K. Wildberger, V.S. Stepanyk, P. Lang, R. Zeller, P.H. Dederichs, Phys. Rev. Lett. 75 (1995) 509.

[340] D.M. Cox, Nanostruct. Mater. 1 (1992) 161.

[341] U. Heiz, R. Sherwood, D.M. Cox, A. Kaldor, J.T. Yates, J. Phys. Chem. 99 (1995) 8730.

[342] M. Haruta, Catal. Today 36 (1997) 153.

[343] B. Hammer, J.K. Nørskov, Nature 376 (1995) 238.

[344] W. Gotschy, K. Vonmetz, A. Leitner, F.R. Aussenegg, Opt. Lett. 21 (1996) 1099.

[345] W. Gotschy, K. Vonmetz, A. Leitner, F.R. Aussenegg, Appl. Phys. B 63 (1996) 381.

[346] K. Eberl, Phys. World (September 1997) 47.

[347] R. Leon, P.M. Petroff, D. Leonard, S. Farard, Science 267 (1995) 1966.

[348] I.M.L. Billas, A. Châtelain, W.A. de Heer, Science 265 (1994) 1682. 